University of Louisville

ThinkIR: The University of Louisville's Institutional Repository

Electronic Theses and Dissertations

$12-2019$

\title{
Laser spectroscopy investigations on molecular and free radicals dynamics.
}

Hamzeh Telfah

University of Louisville

Follow this and additional works at: https://ir.library.louisville.edu/etd

Part of the Physical Chemistry Commons

\section{Recommended Citation}

Telfah, Hamzeh, "Laser spectroscopy investigations on molecular and free radicals dynamics." (2019). Electronic Theses and Dissertations. Paper 3322.

https://doi.org/10.18297/etd/3322

This Doctoral Dissertation is brought to you for free and open access by ThinkIR: The University of Louisville's Institutional Repository. It has been accepted for inclusion in Electronic Theses and Dissertations by an authorized administrator of ThinkIR: The University of Louisville's Institutional Repository. This title appears here courtesy of the author, who has retained all other copyrights. For more information, please contact thinkir@louisville.edu. 


\section{LASER SPECTROSCOPY INVESTIGATIONS ON MOLECULAR AND FREE RADICALS DYNAMICS}

By

Hamzeh Telfah

A dissertation submitted to the Faculty of the College of Arts and Sciences of the University of Louisville in partial fulfillment of the requirements for the degree of

Doctor of Philosophy in Chemistry

Department of Chemistry

University of Louisville

Louisville, Kentucky

December 2019 
Copyright 2019 by Hamzeh Telfah All rights reserved 



\title{
LASER SPECTROSCOPY INVESTIGATIONS ON MOLECULAR AND FREE RADICALS DYNAMICS
}

\author{
By \\ Hamzeh Telfah \\ A Dissertation Approved on
}

October $24^{\text {th }}, 2019$

by the following Dissertation Committee:

Dr. Jinjun Liu

Dissertation Director

Dr. Sergio Mendes

Dr. Sachin Handa

Dr. Lee Thompson 


\section{ACKNOWLEDGMENTS}

Collaborative work, guidance, and help from professors, staff, and friends in the Department of Chemistry, as well as outside the department, are the reasons behind the completion of this dissertation. Support from family and friends is also another major reason. I am grateful for all of those who contributed to helping me during my study years.

First, my acknowledgement to my advisor, Professor Jinjun Liu, for

giving me the opportunity to work on his research labs, where I had the chance to develop my knowledge, skills, and expertise in many fields; laser spectroscopy, chemistry, physics, mechanics, electronics, programming are only examples along with many other entangled sciences and information. Dr. Liu continuously provided advice on my research. Patience is one of the great, most important characters of Dr. Liu.

I would also thank all my Ph.D. committee members: Professor Sergio Mendes, Professor Sachin Handa, and Professor Lee Thompson. They were not only a dissertation committee of mine, but also, they provided a lot of advice and suggestion to work presented in this dissertation. 
My thanks also to our collaborators in the Department of Chemistry and Chemical Biology of Indiana University - Purdue University Indianapolis: Professor Rajesh Sardar and Dr. Meghan Teunis, who provided the experimental samples of the first project in this dissertation.

I also want to thank my friend and lab mate Abdelqader Jamhawi, who helped me to "jump-start" my research in the ultrafast lab. Along with my lab mates Asmaul Reza and Anam paul for their help and support during my work in the high-resolution lab, former group members, Jahangir Alam, Jacob strain, and Yizhou Xie.

Thanks to the staff of the Chemistry Department: Sherry, Carla, Jason, Ben, Renu, and Steve. Along with former staff members, Sabrina and Aaron. Also, I wouldn't forget the Conn Center members, Eunice Salazar and Director Mahendra Sunkara.

Special thanks for Josh in the physics department, where we always find a solution for our mechanical problem

Many thanks for every single person in this university who participated in this dissertation. 
Last but not least, I would like to give a special thanks to my mother, brothers, sisters, and the whole family who unconditionally supported me overseas through all the years of my life.

Special thanks and appreciation to my wife, Angham, who supported me through my years of study. 


\section{DEDICATION}

This dissertation is dedicated to my parents, my wife, and my son 


\title{
ABSTRACT
}

\section{LASER SPECTROSCOPY INVESTIGATIONS ON MOLECULAR AND FREE RADICALS DYNAMICS}

\author{
Hamzeh Telfah
}

October $24^{\text {th }}, 2019$

Studying molecular dynamics and chemical kinetics is important to understand the chemical behavior of renewable energy sources. Laser spectroscopy techniques are powerful tools for the identification and diagnosis of such processes.

In this dissertation, using our laser spectroscopy techniques, biofuels and solar energy were targeted as renewable energy sources study. We have studied the ultrafast exciton dynamics for a series of methylammonium lead bromide $\left(\mathrm{CH}_{3} \mathrm{NH}_{3} \mathrm{PbBr}_{3}\right)$ nanostructures, nanocrystals (NCs, 0D), nanowires (NWs, 1D), and nanoplatelets (NPs, 2D) as a promising solar energy materials. ${ }^{1-2}$ Aided by analysis of UV-visible absorption and photoluminescence spectra, features in the transient absorption (TA) spectra are assigned to different charge carrier processes, time constants of which are determined in fitting the transient kinetics. 
Immediately after photoexcitation, the charge carrier thermalization process occurs within the instrument response function time and results in a quasi-equilibrium distribution of charge carriers. It is followed by charge carrier cooling on a sub-picosecond time scale. The charge carrier recombination process obeys the rate law of a second-order reaction, which suggests that it occurs mainly via the bimolecular nongeminate recombination process. Dependence of the charge recombination rate constant on the initial charge carrier density has also been investigated in fluence-dependence measurements. The sensitivity of the recombination rate constant to initial charge carrier density is different for the three perovskite nanostructures, signifying the strong impact of quantum confinement on exciton dynamics.

Using room-temperature cavity ring-down spectroscopy (CRDS), we obtained the spectra of the $\tilde{\mathrm{A}} \leftarrow \tilde{\mathrm{X}}$ electronic transition of tetrahydrofuranyl peroxy (THFOO•) and tetrahydropyranyl peroxy (THPOO•) radicals. ${ }^{3-4}$ The peroxy radicals were produced by $\mathrm{Cl}$-initiated oxidation of tetrahydrofuran and tetrahydropyran. Quantum chemical calculations of the lowest-energy conformers of all regioisomers of these two peroxy radicals have been carried out to aid the spectral simulation. Conformational identification and vibrational assignment were achieved by comparing the experimentally obtained spectra to the simulated ones. The absence of $\alpha$-THPOO• absorption peaks in the CRD spectrum is attributed to ring-opening due to its weak $\mathrm{Ca}^{\prime} \mathrm{O}$ bond. 


\section{TABLE OF CONTENTS}

ACKNOWLEDGMENTS

DEDICATION vi vi

ABSTRACT vii

LIST OF FIGURES xii

LIST OF TABLES Pix

1 INTRODUCTION 1

1.1 Perovskites solar cells 3

1.1.1 Perovskites Crystal structures $\quad 5$

1.1.2 Perovskites Morphologies and dimensionality 7

$\begin{array}{lll}1.2 & \text { Peroxy radicals } & 7\end{array}$

1.2.1 Peroxy radicals in combustion reaction chemistry 8

$\begin{array}{ll}\text { 1.2.2 Peroxy radicals in atmospheric chemistry } & 10\end{array}$

1.2.3 Reactions of peroxy radicals with $\mathrm{NO}_{2} \quad 12$

$\begin{array}{ll}\text { 1.2.4 Peroxy radicals electronic structures } & 14\end{array}$

2 EXPERIMENTAL $\quad 16$

2.1 Transient Absorption (TA) Spectroscopy 16

$\begin{array}{ll}2.1 .1 & \text { Pump-Probe principal } \\ & 16\end{array}$

$\begin{array}{ll}\text { 2.1.2 Signal possible contributions } & 19\end{array}$

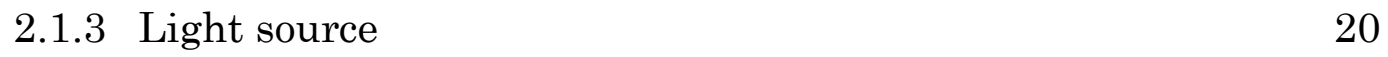

$\begin{array}{lll}\text { 2.1.3.1 Laser head } & 21\end{array}$

2.1.3.2 Wavelength Tuning (frequency conversions) 22

$\begin{array}{lll}\text { 2.1.3.2.1 Pump sources } & 22\end{array}$

2.1.3.2.2 Probe source 23

2.1.4 Experimental setup 23

$2.2 \quad$ Cavity ring-down Principle 26

2.2.1 Absorption Spectroscopy $\quad 26$

$\begin{array}{ll}\text { 2.2.2 CRDS principal } & 27\end{array}$

$\begin{array}{ll}\text { 2.2.3 CRDS cavity alignment } & 31\end{array}$

2.2.4 Radical generation via photolysis 32

2.2.5 Light sources 33

2.2.5.1 Photolysis lasers 33

2.2.5.2 CRD Laser 36

2.2.6 Room-Temperature reaction cell 40

2.2.7 Detection, Timing control, and delay generation 41 
3 ULTRAFAST EXCITON DYNAMICS IN SHAPE CONTROLLED METHYLAMMONIUM LEAD BROMIDE PEROVSKITE NANOSTRUCTURES: EFFECT OF QUANTUM CONFINEMENT ON CHARGE CARRIER RECOMBINATION

3.1 Introduction 44

3.2 Experiment 46

3.3 Results and Discussion $\quad 51$

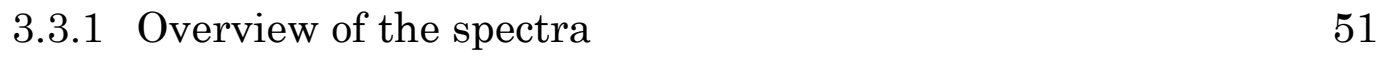

3.3.1.1 Steady-state spectra 51

3.3.1.2 Transient Absorption spectra 56

3.4 Transient Kinetics $\quad 57$

3.4.1 Thermalization and cooling of hot charge carriers 57

3.4.2 Charge recombination process 63

$\begin{array}{lll}3.5 & \text { Pump fluence dependence study } & 68\end{array}$

$\begin{array}{lll}3.6 & \text { The role of trap states } & 78\end{array}$

$\begin{array}{lll}3.7 & \text { Global fitting } & 79\end{array}$

3.8 Conclusions 83

4 DIRECT OBSERVATION OF TETRAHYDROFURANYL (THF-YL) AND TETRAHYDROPYRANYL (THP-YL) PEROXY RADICALS, A BIOFUEL COMBUSTION REACTION INTERMEDIATES, VIA CAVITY RING DOWN SPECTROSCOPY

4.1 Introduction 86

4.2 Experimental 88

$\begin{array}{lll}4.3 & \text { Conformational search } & 92\end{array}$

$\begin{array}{lll}\text { 4.3.1 THFOO• Conformers } & 93\end{array}$

$\begin{array}{ll}\text { 4.3.2 THPOO• Conformers } & 94\end{array}$

4.4 Quantum chemical calculations $\quad 95$

4.5 Intensity calculations and spectral simulations 104

4.5.1 Intensity calculation 104

4.5.2 Spectral simulation (Python analysis) 108

$\begin{array}{lll}4.6 & \text { Results and discussion } & 108\end{array}$

$\begin{array}{ll}\text { 4.6.1 Overview of the spectra } & 108\end{array}$

4.6.2 Artificial dips in the spectra 111

4.6.3 THP and THF absorption cross-section at 193nm 113

4.7 Ring-Opening $\quad 115$

$\begin{array}{lll}4.8 \text { Conclusions } & 121\end{array}$

5 OVERALL CONCLUSION 123

$6 \quad$ FUTURE STUDIES 126 
APPENDIX A:

OPTICAL CAVITY ALIGNMENT AND LASERS

MAINTENANCE

140

A.1 CRDS Cavity alignment

140

A.2 Excimer laser LPX120i maintenance.

APPENDIX B:

PYTHON PROGRAMS FOR DATA ANALYSIS

APPENDIX C:

COPYRIGHT PERMISSIONS

CURRICULUM VITAE

190 


\section{LIST OF FIGURES}

Figure 1.1 Methylammonium lead bromide $\left(\mathrm{CH}_{3} \mathrm{NH}_{3} \mathrm{PbBr}_{3}\right)$ perovskite crystal structure. The figure was reproduced from reference [49] with permission.

Figure 2.1 (A-left) steady-state absorption spectroscopy principle left, (A-right) is an illustration of the transition monitored by probe pulse. (B-left) pump-probe TA spectroscopy with a delay between the two pulses, (Bright) both populations of the ground and excited states are monitored by the probe pulse. (C) introducing the pump-probe delay allows measuring the dynamics related to the excited state after subtracting those for the ground state.

Figure 2.2 (A) Possible transition upon introducing a probe pulse after a pump pulse. Violet arrow represents the pump, while the dashed red arrow represents the ground state bleaching (GSB) signal, and the blue is the stimulated emission (SE). The green arrow represents the excited state absorption (ESA). To the right (B) are the three signals that correspond to the three possible transitions in the left. Red is the GSB, blue is the SE, green is the ESA, and the solid black line is the overall TA signal. 
Figure 2.3 A schematic illustrates our experimental layout. CPA, NOPA, SHG, and TAPPS are shown in the figure. Red arrows represent the fundamental, blue is the SHG output, green is the NOPA output, and the rainbowcolored is whit light. M: mirror, BS: beam splitter, WLX: white light crystal, BBO: NOPA crystal, BD: beam dumber, $v \times 2$ : SHG crystal, FM: flipping mirror, RR: retro reflector, MDS: motorized delay stage. Some optical components like irises and filters and some others are omitted for simplicity. The pump and probe are overlapped in the sample (yellow circle).

Figure 2.4 Illustration of the CRDS principle, when light is injected to an empty cavity (A-left), the laser will bounce back and forth between cavity mirrors, the leaked light intensity is recorded with time (A-right) and result in an exponential decay curve called ringdown curve, the exponential fitting result is the ringdown time. If an absorber is introduced in the cavity (B-left), the leaked intensity decreases, hence ringdown time decrease (BRight). Absorption per pass is calculated from the difference in the ringdown times.

Figure 2.5 A schematic illustrates the CRD light source used. The dye laser is pumped by second harmonics of the Nd:YAG laser then focused on the hydrogen Raman cell to generate IR laser.

Figure 2.6 Schematic illustrating Photolysis Light sources in CRDS experimental setup.

Figure 2.7 Energy diagram illustrating the Raman effect. If molecules were excited, it relaxes back to the ground state with lower frequency (Stokes), or with the same frequency (Rayleigh), or of higher frequency than the input (anti-Stokes).

Figure 2.8 Reaction cell setup and gas flow diagram

Figure 2.9 Timing control and synchronization of all instruments in the current experiment. (A) Represent the TTL out of the PC generated by the LabVIEW program, (B) is the TTL generated by the delay generator, sent to both Nd:YAG and oscilloscope, (C) TTL Out of the and gate, sent to the 
excimer laser. The output of the and gate is generated, adding the TTL signals from A and B, and (D) represent the wavelength change event of the dye laser triggered at the falling edge of the TTL pulses on trace A.

Figure 3.1 TEM images of perovskite nanostructures

Figure 3.2 Histogram of size analysis of $\mathrm{CH}_{3} \mathrm{NH}_{3} \mathrm{PbBr}_{3} \mathrm{NCs}$ determined using the ImageJ software. Approximately $360 \mathrm{NCs}$ were counted for the analysis. The average size was determined to be $2.4 \pm 0.2 \mathrm{~nm}$

Figure 3.3 (a) Steady-state absorption and PL spectra. Orange and navy vertical lines indicate the band-edge absorption peaks and the PL peaks, respectively. (b) 2D pseudocolor plots of TA spectra of perovskite NCs, NWs, and NPs. Vertical dash lines indicate peak centers $(\mathrm{hv} 1<\mathrm{hv} 2$ $<$ hv3 $<$ hv4). Note that the maxima of signals are displaced from the peak centers due to interference between adjacent peaks. $\mathrm{mOD}=$ milli-optical density .

Figure 3.4 Transient kinetics of (a) NCs, (b) NWs, and (c) NPs at four-probe photon energies. Thin lines are simulations using parameters determined in the global fitting.

Figure 3.5 (a) Comparison between TA spectra with two pump photon energies: $3.2 \mathrm{eV}$ (top) and $2.5 \mathrm{eV}$ (bottom). The pump-probe delay times $(\Delta \mathrm{t})$ are between 0 and 1 ps. Vertical dashed lines indicate the center of the photoinduced absorption (PIA) signal ( $\left.h v_{1}\right)$. (b) Comparison between transient kinetics at $h v_{1}$ with the two pump photon energies. Note that the PIA signal is absent with $\mathrm{h} v_{\text {pump }}=2.5 \mathrm{eV}$.

Figure 3.6 Energy diagram and photoinduced processes in perovskite nanostructures. Straight arrows mark the photon energies of the pump beam and the peaks in the TA spectra. Wavy arrows indicate charge carrier relaxation processes. Thick and thin solid horizontal lines are the original and renormalized band gaps, respectively. $\mathrm{f}(\mathrm{T})$ : charge carrier distribution

Figure 3.7 Decay curve at hv2 of the TA spectrum of NWs at 20 $\mu \mathrm{J} / \mathrm{cm}^{2}$ pump fluence fit to the concentration profile of 
the reactant of (a) a first-order reaction (for geminate recombination); (b) a second-order reaction (for nongeminate recombination), and (c) a third-order reaction (for Auger recombination) along with residuals. The second-order reaction model gives the best fit, while systematic errors are introduced using the other two. Similar observations have been made for other nanostructures or at another pump fluences.

Figure 3.8 Transient kinetics of (a) NCs, (b) NWs, and (c) NPs at $\mathrm{hv}_{3}$ under different pump fluences. The TA signals are normalized to its maximum at $\sim 0.5 \mathrm{ps}$.

Figure 3.9 Charge-carrier cooling rate constants $\left(\mathrm{k}_{\mathrm{c}}\right)$ for NCs (blue squares), NWs (red circles) and NPs (green triangles) at different initial charge carrier density $\left[\mathrm{n}_{0}\right]$. Error bars represent one standard deviation (RMS) of fitting the decay signal at hvs to an exponential function. Solid lines are fittings to a linear function. Data points are weighted by $1 / \sigma^{2}$ in the fittings.

Figure 3.10 Transient kinetics of (a) NCs, (b) NWs, and (c) NPs at $\mathrm{hv}_{2}$ under different pump fluences. Thin lines are fittings to Equation 3.1.

Figure 3.11 (a) Half-time of charge carrier recombination $\left(\tau_{1 / 2, r}\right)$ and (b) maximum SF signal at $\mathrm{hv}_{2}$ under different pump fluences $\left(\mathrm{F}_{\text {pump }}\right)$ for NCs (blue squares), NWs (red circles) and NPs (green triangles).

Figure 3.12 Effective charge carrier recombination rate constant $\left(\mathrm{k}_{\mathrm{r}}\right)$ for NCs (blue squares), NWs (red circles) and NPs (green triangles) at different initial charge carrier density $\left[\mathrm{n}_{0}\right]$. Error bars represent five times standard deviation (RMS) of fitting the decay of SF/SE signal at $\mathrm{hv}_{2}$ to Equation 3.1. Solid lines are fittings to equation 3.3.

Figure 3.13 Fluorescence lifetimes for the three nanostructures, decay fitting results in a nano-second decay lifetime, which means there are no trap states in the system.

Figure 3.14 Simulation of TA spectra of (a) NCs, (b) NWs, and (c) NPs at different delay times $(\Delta t=0.4,1,10,400 \mathrm{ps})$ using parameters determined in the global fitting (Table 3.4). Symbols are the experimental spectra. Solid lines are 
simulated spectra. Dashed lines are simulated components of TA spectra with different peak centers.

Figure 4.1 Lowest-energy conformers of the THFOO- radical, ground-state geometries optimized by DFT calculations. Hydrogen atoms are omitted for clarity

Figure 4.2 Optimized geometries using UB3LYP calculated for twisted boat conformers

Figure 4.3 PES scan along OCCH Dihedral angle of the B-Ax conformers

Figure 4.4 Lowest-energy conformers of the THPOO• radical. All such conformers have the "chair" ring structure. Ground state geometries optimized by DFT calculations. Hydrogen atoms are omitted for clarity. Note that $\mathrm{B}-\mathrm{Ax}-$ $\mathrm{T}$ is a transition state

Figure 4.5 Simulated spectra of a (left) and B (right) THFOO• conformers. The overall spectrum is calculated as the summation of spectra of all conformers weighted by their relative populations. All spectra are offset for clarity

Figure 4.6 Simulated spectra of a (Top) and $B$ and $\gamma$ (Bottom) THPOO• conformers. The overall spectrum is calculated as the summation of spectra of all conformers weighted by their relative populations. All spectra are offset for clarity

Figure 4.7 CRD spectra of THF (top) and THP (bottom) precursors (red traces) in comparison with those of the THFOO• and THPOO• radicals (black traces), respectively. Radical spectra are offset for clarity. Dips labeled with asterisks are due to strong precursor absorption.

Figure 4.8 Decay kinetics of THFOO• (top) and THPOO• (bottom) radicals measured at different peaks in their CRD spectra. The decay curves are fit to a bi-exponential function with fit time constants given in the figures.

Figure 4.9 CRD spectra recorded with $5 \mu \mathrm{s}$ (top) and $15 \mathrm{~ms}$ (bottom) delay times between the photolysis and the CRD laser 
pulses $(\Delta \mathrm{t})$. Absorption peaks are assigned to the $\mathrm{OO}$ stretch of THPOO• radicals.

Figure 4.10 CRD spectra obtained by $266 \mathrm{~nm}$ (black) and $193 \mathrm{~nm}$ (red) photolysis of (a) THF and (b) THP in the presence of excessive $\mathrm{O}_{2}$.

Figure 4.11 (a) CRD spectrum of the THPOO radical. (b) The simulated spectrum of $\alpha$-THPOO $\cdot$. (c) Simulated spectrum of B-THPOO• (d) Simulated spectrum of $\gamma-$ THPOO•. Thin lines in (b), (c), and (d) are simulated spectra of conformers, while the thick line is their superposition weighted by concentrations. (e) Superposition of the simulated spectra of all regioisomers with a weight of $76.0 \%$ for $\alpha$-THPOO•, $16.0 \%$ for $8-\mathrm{THPOO} \cdot$, and $8.0 \%$ for $\gamma$-THPOO• Peaks predicted in the region of $6600-7000 \mathrm{~cm}^{-1}$ due to the $\alpha-$ regioisomer is absent in the experiment spectrum.

Figure 4.12 (a) CRD spectrum of the THFOO radical. (b) The simulated spectrum of $\alpha$-THFOO•. (c) The simulated spectrum of B-THFOO- Thin lines in (b) and (c) are simulated spectra of conformers, while the thick line is their superposition weighted by concentrations. (d) Superposition of the simulated spectra of both regioisomers with a weight of $82.5 \%$ for $\alpha-\mathrm{THFOO} \cdot$ and $17.5 \%$ for $B-$ THFOO•

Figure 4.13 (a) CRD spectrum of the THPOO• radical. (b) Simulated spectra of B-THPOO. (c) Simulated spectra of $\gamma-$ THPOO•. Thin lines in (b) and (c) are simulated spectra of conformers, while the thick line is their superposition weighted by concentrations. (d) Superposition of the simulated spectra of $B-$ and $\gamma$-THPOO - regioisomers with a weight ratio of $2: 1$.

Figure A.1 Beam paths diagram for both IR and HeNe lasers. Beams are shifted for clarity. (CRDM) Cavity Ring-Down Mirror, (FM) Flipping Mirror, (I) Iris, (M) Mirror, (LPF) Long Pass Filter

Figure A.2 Beam alignment tool machined using an acrylic disk to match the cavity arm end. 
Figure A.3 Alignment of the IR spot on the IR card using the Beam alignment tool, dashed line is the footprint of the IR spot if it is centered on the axis of the cavity and iris $\mathrm{I}_{4}$. A is the vertical centering, $\mathrm{B}$ is the horizontal centering and $\mathrm{C}$ is aligned spot

Figure A.4 Visualizing the IR spot on the Irises using an IR viewer. the green ring is what is seen when the IR beam is centered on the iris, and the iris is partially closed

Figure A.5 Wedged mirror reflection spots on cavity card, reflection from the wedged surface is rotating while from the HR coated surface is almost stationary.

Figure A.6 Ring structure that is seen on the cavity alignment card. (A) comparison between centered and off-centered reflection, (B) picture of the centered ring structure of reflection

Figure A.7 Reflection spot if ring structure was not observed

Figure A.8 First and second round reflections when both CRDM are mounted. The second reflection is twice further than the first and travels twice faster.

Figure B.1 Flow chart for the three python programs used in data analysis, gaussian parameter extraction (A), FCFs Calculations (B), and spectra convolution (C). 


\section{LIST OF TABLES}

Table 2.1 Gas mixture ratios for ArF excimer laser, output $193 \mathrm{~nm}$

Table 3.1 Peak centers in UV-visible absorption (Abs.) and photoluminescence (PL) spectra, photoluminescence quantum yields $(\Phi F L)$, and radiative lifetimes $(\tau)$ of $\mathrm{CH}_{3} \mathrm{NH}_{3} \mathrm{PbBr}_{3}$ perovskite nanostructures.

Table 3.2 Charge carrier cooling rate constants at the zero-density limit $\left(k_{c}^{(0)}\right)$ and linearity coefficients $\mathrm{X}_{\mathrm{c}}$ of $\mathrm{CH}_{3} \mathrm{NH}_{3} \mathrm{PbBr}_{3}$ perovskite nanostructures determined in fitting the transient kinetics

Table 3.3 Charge-carrier recombination rate constants at the zerodensity limit $\left(k_{\mathrm{r}}^{(0)}\right)$ and linearity coefficients $\mathrm{Xr}$ of $\mathrm{CH}_{3} \mathrm{NH}_{3} \mathrm{PbBr}_{3}$ perovskite nanostructures determined in fitting the transient kinetics.

Table 3.4 Peak centers (hv), linewidths $(\gamma)$, and time constants of charge carrier cooling $\left(\tau_{c}\right)$ and recombination $\left(\tau_{1 / 2, \mathrm{r}}\right)$ processes determined in the global fittings of TA spectra of the perovskite nanostructures.

Table 4.1 Calculated relative energies, Boltzmann distribution factors (at $300 \mathrm{~K}$ ), relative populations, and the adiabatic $\widetilde{A}-\widetilde{X}$ excitation energies $\left(\Delta E^{\widetilde{A}-\widetilde{X}}\right)$ for the lowest-energy conformers of THFOO•. Boltzmann distribution factors are normalized for each Regio isomer. Population Ratio for $\alpha$ and $B$ was set to $82.5-17.5$ 
Table 4.2 Calculated relative energies, Boltzmann distribution factors (at $300 \mathrm{~K}$ ), relative populations, and the adiabatic $\widetilde{A}-\widetilde{X}$ excitation energies $\left(\Delta E^{\widetilde{A}-\widetilde{X}}\right)$ for the lowest-energy conformers of THPOO•. Boltzmann distribution factors are normalized for each Regio isomer. Population Ratio for $\alpha$ and $B$ and $\gamma$ was set to $0: 2: 1$

Table 4.3 Relative energies, Boltzmann factors, and relative populations of THFOO- conformers calculated at the B3LYP/6-31+G(d) level of theory and using the CBSQB3 composite method

Table 4.4 Relative energies, Boltzmann factors, and relative populations of THPOO $B$ and $\gamma$ conformers calculated at the B3LYP/6-31+G(d) level of theory and using the CBS-QB3 composite method

Table 4.5 Relative energies of selected THPOO conformers with the twist-boat ring structure.

Table 4.6 Bond dissociation energy (BDE) of the lowest-energy conformers of all regioisomers of THPOO• and THFOO• using the CBS-QB3 method. 


\section{CHAPTER 1}

\section{INTRODUCTION}

Renewable and sustainable energy has gained the greatest interest in the research since petroleum consumption is increasing, and fossil fuels are eventually going to be consumed. Renewable Energy Sources (RES) are clean sources of energy, including hydropower, geothermal, wind, biofuels, and solar energy, mainly used for heating and power. The worldwide percentage is suggested to reach up to $80 \%$ by 2100.5 Today's high demands on energy show the need for the implementation of technologies that use RES to make sure we achieve a sustainable future. Among the various above-mentioned RES, we choose two categories to study using our laser spectroscopy techniques; solar energy and biofuels sources. For such systems, laser spectroscopy techniques are powerful tools for the identification and diagnosis of chemical reaction intermediates, as well as studying molecular dynamics and chemical kinetics.

For the solar energy source, we have studied the ultrafast exciton dynamics for Methylammonium Lead Bromide Perovskite Nanostructures as a promising photovoltaic device material and investigated the effect of quantum confinement on charge carrier recombination using transient absorption spectroscopy. 
Metal-halide perovskites are considered promising light absorbers for photovoltaic applications, ${ }^{6}$ as well as materials in light-emitting diodes, ${ }^{7}$ photodetectors,${ }^{8}$ and lasers. ${ }^{9}$ They possess an array of favorable photoelectric properties including strong absorption across the visible range, very low exciton binding energy $(\sim 16 \mathrm{meV}$ at low temperatures $),{ }^{10}$ high charge carrier mobility, long diffusion length $(>1 \mu \mathrm{m})$ for both electrons and holes, ${ }^{11}$ and low charge carrier recombination rate. Additionally, a unique property of perovskites is that their bandgaps are controllable by the halide content so that they can be tuned and optimized for the solar spectrum. ${ }^{12}$ Power conversion efficiencies (PCEs) of thin-film perovskite-based photovoltaic cells have increased from about $3.8 \%$ to $22.1 \%$ in recent years. ${ }^{13-14}$ Higher efficiencies may be achieved by bandgap tuning via structural modifications of the materials to induce quantum confinement or tunneling, ${ }^{15}$ which lead to an increase and decrease of the bandgap, respectively. Another important approach to higher PCEs is via suppression of trap states and reduction of trap-assisted recombination, ${ }^{16-19}$ Ultimately, increasing $\mathrm{PCE}$ relies on optimization of photoinduced processes in photovoltaic materials.

Another class of RES is the biofuels. The development and utilization of biofuels are proposed as a source of renewable energy intended to reduce the existing dependence on petroleum-derived fuels. One type of biofuels is lignocellulosic biofuels, mainly contains cellulose, hemicellulose, and lignin. ${ }^{20}$ Cellulose is composed of glucopyranose (in the form of a $\left(\mathrm{C}_{6} \mathrm{H}_{10} \mathrm{O}_{5}\right)_{\mathrm{n}}$ polymer), 
and hemicellulose of $\mathrm{C}_{5} / \mathrm{C}_{6}$ polysaccharides, Tetrahydropyran (THP) and tetrahydrofuran (THF) is the base structure of monosaccharides of hemicellulose, and themselves are a lignocellulosic-derived biofuels. ${ }^{21}$ The utilization of biofuels, lignocellulosic derived, is most practical in blends with conventional fuels and integration as blending components require fundamental insight into the underlying autoignition chemistry.

Understanding the mechanism of combustion reactions is key for advancing internal combustion engine design, improving the efficiency of ignition chemistry reactions, and reducing pollutant formation.

It is critical to detect and identify transient reactive chemical intermediates, particularly free radicals, to model the extremely complicated chemical kinetics of combustion processes. ${ }^{22-23}$ Many intermediates in the combustion of fuels, however, remain unidentified. For instance, although the central role of carbonyl oxides $\left(\mathrm{CH}_{2} \mathrm{OO}\right)$ proposed by Criegee in 1949 as key intermediates in the ozonolysis of alkenes, ${ }^{24}$ they were not directly observed in experiment until recently. ${ }^{25-28}$ Because direct detection of these gaseous intermediates was unavailable, the previous understanding of their reactions, derived from indirect experimental evidence, had great uncertainties

\subsection{Perovskites solar cells}

The first perovskite-based solar cell was fabricated in 2012, with power conversion efficiency (PCE) of about 9\%. ${ }^{29}$ Since then, perovskite-based solar 
cells showed a rapid increase in their PCEs to reach more than $23 \%$ by 2018. ${ }^{6,13,30-34}$ This makes the perovskite-based solar cells subjected to many studies. These studies include investigating new structures using different metals, legends, and halogens - and their photophysical properties.

Metal-halide perovskites are promising light absorbers for photovoltaic applications, ${ }^{6,29-30,35-37}$ as well as materials in light-emitting diodes, ${ }^{7}$ photodetectors, ${ }^{8}$ and lasers. ${ }^{9,38}$ They possess an array of favorable photoelectric properties including strong absorption across the visible range, very low exciton binding energy $(\sim 16 \mathrm{meV}$ at low temperatures $),{ }^{10}$ high charge carrier mobility, long diffusion length $(>1 \mu \mathrm{m})$ for both electrons and holes, ${ }^{11}$ and low charge carrier recombination rate. Perovskites are also relatively cheap. ${ }^{36}$

Additionally, a unique property of perovskites is that their bandgaps are controllable by the halide content so that they can be tuned and optimized for the solar spectrum. ${ }^{12}$

Ultimately, increasing PCE relies on the optimization of photoinduced processes in photovoltaic materials. Transient absorption (TA) spectroscopy is a powerful tool for the study of such processes. In particular, TA spectroscopy has been employed to investigate photoinduced processes in perovskite thin films, ${ }^{11,39-42}$ nanostructures, ${ }^{43-44}$ and perovskite solar cell interfaces. ${ }^{39,45-46}$ 


\subsubsection{Perovskites Crystal structures}

Perovskite is a type of a molecular structure, with general molecular structure of $\mathrm{AMX}_{3}$, where $\mathrm{A}$ is a large cation, which could be an organic cationic compound, $\mathrm{M}$ is a metal ion, usually lead ion $\left(\mathrm{Pb}^{2+}\right)$, while $\mathrm{X}$ is a halogen anion, the crystal structure of methylammonium lead bromide perovskite is illustrated in figure 1.1. Different combinations of A, M, and X lead to different properties, mainly photophysical properties, such as charge carrier mobility, optical bandgap, and diffusion lengths, etc.

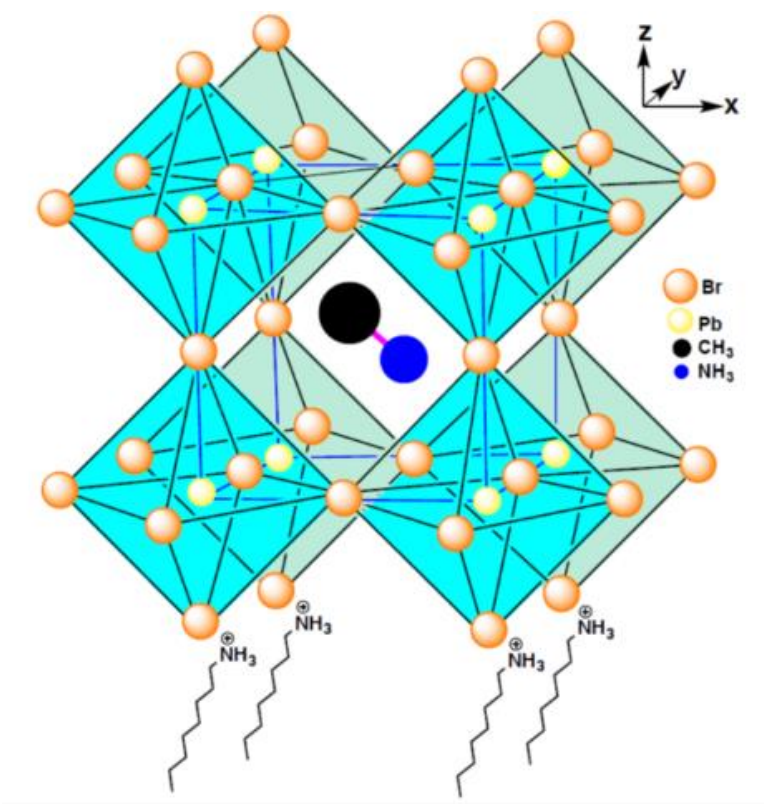

Figure 1.1. Methyl ammonium lead bromide $\left(\mathrm{CH}_{3} \mathrm{NH}_{3} \mathrm{PbBr}_{3}\right)$ perovskite crystal structure. Figure was reproduced from reference [49] with permission.

In methylammonium lead-Bromide perovskites, $\mathrm{A}$ is a methylammonium cation $\left(\mathrm{CH}_{3} \mathrm{NH}_{3}{ }^{+}\right)$that are encased by $\mathrm{PbBr}_{6}{ }^{4-}$ units where $\mathrm{M}$ is $\mathrm{Pb}^{2+}$ and $\mathrm{X}$ is $\mathrm{Br}^{-}$. The lead is in the center, and the six halogen anions 
surrounding the metal form an octahedral structure. Hybrid perovskites are usually deposited as polycrystalline thin films with variable mesoscale morphology and grain sizes ranging from tens to thousands of nanometers depending on the growth conditions. ${ }^{47-48}$

Higher efficiencies may be achieved by bandgap tuning via structural modifications of the materials to induce quantum confinement or tunneling, ${ }^{15}$ which leads to an increase and decrease of the bandgap, respectively. Another important approach to higher PCEs is via suppression of trap states and reduction of trap-assisted recombination, also known as the Shockley-Read-Hall process. ${ }^{16-19,31}$ Both optimization mechanisms can be implemented in nanostructures whose size, shape, and surface quality can be controlled by sample preparation time and conditions. ${ }^{16}$

Perovskites, in general, had several issues that limit their use in several applications. Degradation of perovskites is possible if exposed to humidity, oxygen, or heated. This causes an issue of long-term stability. Our perovskite samples were stored under normal laboratory conditions in darkness. The absorption and PL peak positions and shapes was stable, and no changes were observed for almost two months. Moreover, during this period, only a $4 \%$ decrease in $\phi$ was observed. ${ }^{49}$ However, other issues remain and still need to be addressed, such as finding a suitable replacement for lead ions (toxic lead compounds problem). 


\subsubsection{Perovskites Morphologies and dimensionality}

The Octahedral perovskite structure can form different structural shapes in bulk materials and nanostructures. Morphology and grain size, along with other several properties, are key factors in perovskite thin-film based devices PCEs. Varying sizes and shapes of those structures allow controlling their properties. Thus, interest in such morphologies is increasing. These lowdimension perovskites are in Nanoscale $(<100 \mathrm{~nm})$ and include several shapes, like crystals, wires, and platelets. There are more variations, such as cubes or rods. ${ }^{49}$ However, these several shapes can be classified into three main classes; zero-dimensional perovskites Nanostructures (the structure is confined into 3 dimensions) usually called Nano-Crystals ( $\mathrm{NCs}$ ), one dimensional structure (the structure is confined into 2 dimensions) usually called Nano-Wires (NWs) and two dimensional structures (the structure is confined into 1 dimension) usually called Nano-platelets $(\mathrm{NPs}){ }^{1}$

\subsection{Peroxy Radicals}

The chemistry peroxy (ROO•) radicals were subjected to studies since they are one of the most important reaction intermediates, especially in lowtemperature combustion reactions as well as atmospheric chemistry. ${ }^{50-51}$ They are considered as key intermediates in the atmospheric chemistry reactions, as well as in low-temperature combustion of fuels. 
The formation of peroxy radicals in internal combustion reactions starts with the addition of $\mathrm{O}_{2}$ to a hydrocarbon radical $(\mathrm{R} \bullet)$, which initiates the chain reactions. ${ }^{52-53}$ While in the atmosphere, reactions initiated when hydroxyl radicals $(\cdot \mathrm{OH})$ attacks the hydrocarbons, which are biogenic or anthropogenic hydrocarbons and form alkyl radicals, then the addition of oxygen leads to the production of peroxy radicals. Also, in the atmosphere, peroxy radicals are converted to alkoxy radicals $(\mathrm{RO} \bullet)$ mainly via reaction with $\mathrm{NO},{ }^{54-55}$ in addition to entangled chemical reactions involving $\mathrm{NO}_{2}$, ozone, and several other active species. To understand such complicated reactions, one has to be able to detect and determine the concentration of these species, which means, one have to obtain a spectrum of these species. ${ }^{56}$

\subsubsection{Peroxy radicals in combustion reaction chemistry}

Peroxy radicals are not only key components in atmospheric chemistry but also low-temperature internal combustion reactions. In combustion reactions of fuels, peroxy radicals are considered the key reaction intermediate in the whole mechanism. ${ }^{57-61}$ Oxidation of hydrocarbons at low temperatures $(\mathrm{T}=700 \sim 800 \mathrm{~K})$ is a critical process that affects the efficiency of an internal combustion engine. The reaction mechanisms must be understood to improve the efficiencies of engines as well as reduce emissions to the atmosphere. 
The first step in generating the peroxy radicals by the addition of Oxygen to the alkyl radicals $(\mathrm{R} \bullet)($ see Eq. 1.1$)$, this reaction occurs via a threebody reaction, where the third body removes the excess energy.

$$
\mathrm{R} \bullet+\mathrm{O}_{2}+\mathrm{M} \rightarrow \mathrm{ROO} \bullet+\mathrm{M}
$$

Where $\mathrm{M}$ is the third body.

Through a cyclic transition state, peroxy radicals can isomerize, where hydrogen from one of the carbons is transferred to the $\mathrm{OO} \bullet$ moiety, which forms an alkyl radical $(\bullet \mathrm{QOOH})$, the alkyl branch is now called $\mathrm{Q}$ to distinguish from original radicals (see Eq. 1.2).

$$
\mathrm{H}-\mathrm{ROO} \bullet \leftrightarrow \bullet \mathrm{QOOH}
$$

Upon further addition of $\mathrm{O}_{2}$ to the $\cdot \mathrm{QOOH}$, another peroxy radicals can be formed $(\cdot \mathrm{OOQOOH}$ radical $)$,

$$
\mathrm{O}_{2}+\cdot \mathrm{QOOH}+\mathrm{M} \rightarrow \cdot \mathrm{OOQOOH}+\mathrm{M}
$$

The further formed radical decomposes to produce peroxide and (HOO•) radicals. An alkoxy radical (RO•) and a hydroxy radical $(\mathrm{OH} \bullet)$ are formed upon thermal decomposition of peroxy radicals, and $\mathrm{OH} \bullet$ initiates the chain reaction again by reacting with another hydrocarbon, see equations 1.4 and 1.5.

$$
\begin{aligned}
& \mathrm{ROOH} \rightarrow \mathrm{RO} \bullet+\cdot \mathrm{OH} \\
& \bullet \mathrm{OH}+\mathrm{RH} \rightarrow \mathrm{R} \bullet+\mathrm{H}_{2} \mathrm{O}
\end{aligned}
$$


The type of the hydrogen atoms present, i.e., primary, secondary, or tertiary governs the rate of formation of $\bullet \mathrm{QOOH}$ radicals, or in other words, the more the acidic hydrogen, the higher the chance of abstraction and forming -QOOH radicals. ${ }^{62}$

The formation of the $\bullet \mathrm{QOOH}$ species leads to a chain reaction, in which more $\cdot \mathrm{OH}$ radicals are produced. This will lead to an increase in radical concentration (see Eq. 1.4). Hence, results in a process so-called "autoignition process", (AKA engine knocking), this process decreases the efficiency of an internal combustion engine. $57-58$

To decrease engine knock phenomena, reaction mechanisms must be understood to reduce peroxy radical isomerization to $\bullet \mathrm{QOOH}$. This also means that understanding structures (i.e., conformers and isomers) is important to reduce the rate of isomerization of peroxy radicals to $\bullet$ QOOH radicals. ${ }^{63-65}$

\subsubsection{Peroxy radicals in atmospheric chemistry}

Peroxy radicals are not only reactive species in the internal combustion reaction, but they entangle very complicated chemical reactions that occur in the atmosphere. They are considered one of the most important species in the atmosphere, along with Nitrogen dioxide and ozone.

Volatile organic compounds (VOCs), are the main source of the peroxy radicals in the atmosphere, more than $90 \%$ of these VOCs are from biogenic sources, i.e., natural sources. The remaining portion is from anthropogenic 
sources like transportation, combustions, factories, and other human-made sources. ${ }^{66}$ The production of peroxy radicals is initiated by the reaction of these VOCs with $(\cdot \mathrm{OH})$ radicals, which is considered as the most abundant radical in the troposphere.

If produced in the atmosphere, peroxy radicals would cause lots of consequences. First, peroxy radicals undergo a chain of reactions that finally produces $(\cdot \mathrm{OH})$ radicals and alkoxy radicals $(\mathrm{RO} \bullet)$. Which, in turn, further react with oxygen and form $\mathrm{HOO} \cdot$ radicals and carbonyl compounds. Also, the $\mathrm{HOO} \cdot$ radical can react with $\mathrm{NO} \cdot$ to produce $\bullet \mathrm{OH}$ radicals. See Eqs. 1.6 and 1.7 .

$$
\begin{aligned}
& \mathrm{RO} \bullet+\mathrm{O}_{2} \rightarrow \mathrm{R}^{\prime} \mathrm{C}=\mathrm{O}+\mathrm{HOO} \bullet \\
& \mathrm{HOO} \bullet+\mathrm{NO} \bullet \rightarrow \mathrm{NO}_{2}+\cdot \mathrm{OH}
\end{aligned}
$$

$\cdot \mathrm{OH}$, produced in reaction 1.7 , leads to further chemical reactions in the atmosphere. The produced $\bullet \mathrm{OH}$ radicals can participate in several processes in the atmosphere. If migrated to the stratosphere, it will be involved in the decomposition of ozone $\left(\mathrm{O}_{3}\right)$.

$$
\cdot \mathrm{OH}+\mathrm{O}_{3} \rightarrow \mathrm{HOO} \bullet+\mathrm{O}_{2}
$$

Which causes the famous Ozone depletion problem and increase UV radiation on the earth. Second, peroxy radicals interrupt the equilibrium of $\mathrm{NO}_{\mathrm{x}}$, i.e., 
$\mathrm{NO} \bullet \leftrightarrow \mathrm{NO}_{2} \bullet$ in the troposphere, where it increases the production of ozone. In this case, ozone is considered a pollutant.

Moreover, peroxy radicals react with $\mathrm{NO}_{2}$ and form alkyl peroxy nitrates $\left(\mathrm{RO}_{2} \mathrm{NO}_{2}\right)$, which is considered as reservoirs for $\mathrm{NO}_{2}$.

\subsubsection{Reactions of peroxy radicals with $\mathrm{NO}_{2}$}

Another major contribution of peroxy radicals in the tropospheric chemistry are the reactions with $\mathrm{NO}_{2} \bullet$, where it produces peroxy-nitrates $\left(\mathrm{RO}_{2} \mathrm{NO}_{2}\right)$.

$$
\mathrm{RO}_{2}+\mathrm{NO}_{2} \rightarrow \mathrm{RO}_{2} \mathrm{NO}_{2}
$$

Acyl peroxy nitrates, a class of peroxy nitrates, have very long lifetimes at cold temperatures (at the north and the south poles of the globe, lifetimes could be as long as weeks), which act as a reservoir for $\mathrm{NO}_{2}$ until they thermally decompose into peroxy and nitrogen dioxide.

$$
\mathrm{RO}_{2} \mathrm{NO}_{2} \stackrel{\Delta}{\longrightarrow} \mathrm{RO}_{2}+\mathrm{NO}_{2}
$$

Thus, they are considered as a reservoir for $\mathrm{NO}_{2}$ over wider areas. ${ }^{67}$

Nitrogen dioxide is involved as a central reactant in free radicals cycling as well as ozone production, where the latter is considered as a pollutant in the troposphere. 
When exposed to UV light, $\mathrm{NO}_{2}$ decomposes into $\mathrm{NO}$ and atomic Oxygen. Which, in turn, reacts with molecular Oxygen, and form Ozone via a 3-body reaction mechanism. ${ }^{68}$

$$
\begin{gathered}
\mathrm{NO}_{2} \stackrel{\mathrm{h} v}{\longrightarrow} \mathrm{NO} \bullet+\mathrm{O} \bullet \\
\mathrm{O} \bullet+\mathrm{O}_{2} \rightarrow \mathrm{O}_{3} \\
\mathrm{NO} \bullet+\mathrm{O}_{3} \rightarrow \mathrm{NO}_{2}+\mathrm{O}_{2}
\end{gathered}
$$

The produced Ozone can further react with NO• in the first step and produce $\mathrm{NO}_{2}$ again, which means, as a net result, ozone will not be produced in the troposphere. However, this is true only if other free radicals do not exist. In fact, the presence of peroxy radicals interrupts the above mechanism. It was found that the reaction rate of the NO with peroxy radicals is at least 100 times faster than the reaction with Ozone, ${ }^{68}$ i.e., more than $99 \%$ of the produced $\mathrm{NO} \cdot$ will reproduce $\mathrm{NO}_{2}$ again, and the production of ozone will be more pronounced.:

$$
\begin{aligned}
& \mathrm{NO} \bullet+\mathrm{RO}_{2} \bullet \rightarrow \mathrm{NO}_{2} \bullet+\mathrm{RO} \bullet \\
& \mathrm{NO}_{2} \stackrel{\mathrm{h} \nu}{\longrightarrow} \mathrm{NO} \cdot+\mathrm{O} \cdot \\
& \mathrm{O} \bullet+\mathrm{O}_{2} \rightarrow \mathrm{O}_{3}
\end{aligned}
$$




\subsubsection{Peroxy radicals electronic structures}

The electronic structure of the peroxy radical was well studied.69-76 In general, most peroxy radicals share similar features of the electronic structure; this is mainly because the electron promotion in peroxy radicals happens on the same moiety, which is the $\mathrm{OO}$ moiety. Thus, we will discuss the electronic structure of the simplest peroxy radical, methyl peroxy radical $\left(\mathrm{CH}_{3} \mathrm{OO} \bullet\right)$, as an example.

The electronic structure of methyl peroxy radical is well discussed in by Jafri et al. ${ }^{74}$ The first excited electronic state ( $\tilde{A}$ state) was found to be bound state, while the second excited state ( $\tilde{\mathrm{B}}$ state) was a dissociative state.

Previously, UV absorption spectroscopy was used to monitor the peroxy radicals, ${ }^{77-79}$ targeting their $\tilde{\mathrm{B}} \leftarrow \tilde{\mathrm{X}}$ absorption. The advantage of monitoring this transition is that it has a large absorption cross section, which makes monitoring kinetics much easier than if it has low absorption. However, since the $\tilde{\mathrm{B}}$ state is a dissociative state, the resulting spectrum for almost all peroxy radicals is a broad featureless absorption mainly due to the lifetime broadening associated with such state, this makes the use of UV transition not suitable for monitoring the kinetics of peroxy radical, especially when self-reactions of peroxy do exist, and several peroxies do exist in the sample, which is very

common in the gas phase spectroscopy, this makes peroxy radicals indistinguishable from each other since they share similar structures. 
On the other hand, the $\tilde{A}-\tilde{X}$ transition is a better choice for the purpose. The $\tilde{A}-\tilde{X}$ transition falls NIR, and since $\tilde{A}$ state is a bound state, unlike $\tilde{B}$ state, this means that the absorption band in this region will have a unique structure for each peroxy radical, which makes distinguishing peroxy radicals from each other possible, and allows following the kinetics of the targeted radicals. However, the $\tilde{\mathrm{A}}-\tilde{\mathrm{X}}$ transition is forbidden, which means that the cross section for this transition significantly smaller than that for $\tilde{B}-\tilde{X}$ transition. ${ }^{80}$ Unfortunately, traditional absorption techniques are not sufficient in this case, but special techniques are needed. 


\section{CHAPTER 2}

\section{EXPERIMENTAL}

\subsection{Transient Absorption (TA) Spectroscopy}

\subsubsection{Pump-Probe principal}

In normal absorption, if a laser pulse shined to the sample and the transmission is recorded, it represents the ground state population. (See figure 2.1 A). While In the pump-probe experiment, usually, the excited state dynamics are the target of the study, like in our experiment. Thus, the samples have to be prepared in the targeted excited state before measuring transmission, and this is achieved by using a strong laser pulse shined to the sample. This pulse called the "pump" and used only to prepare the sample in the excited state. It gets dumbed after that. Then, some of the samples will have a population in the targeted excited state. Therefore, another laser pulse is shined to the sample after a certain time, and the transmission of this pulse is recorded. The second pulse is now called "Probe" and used to monitor the population of the samples after "pumping" with the first pulse. The pump and the probe pulses must overlap spatially and temporally in the sample. (See figure $2.1 \mathrm{~B})$. 

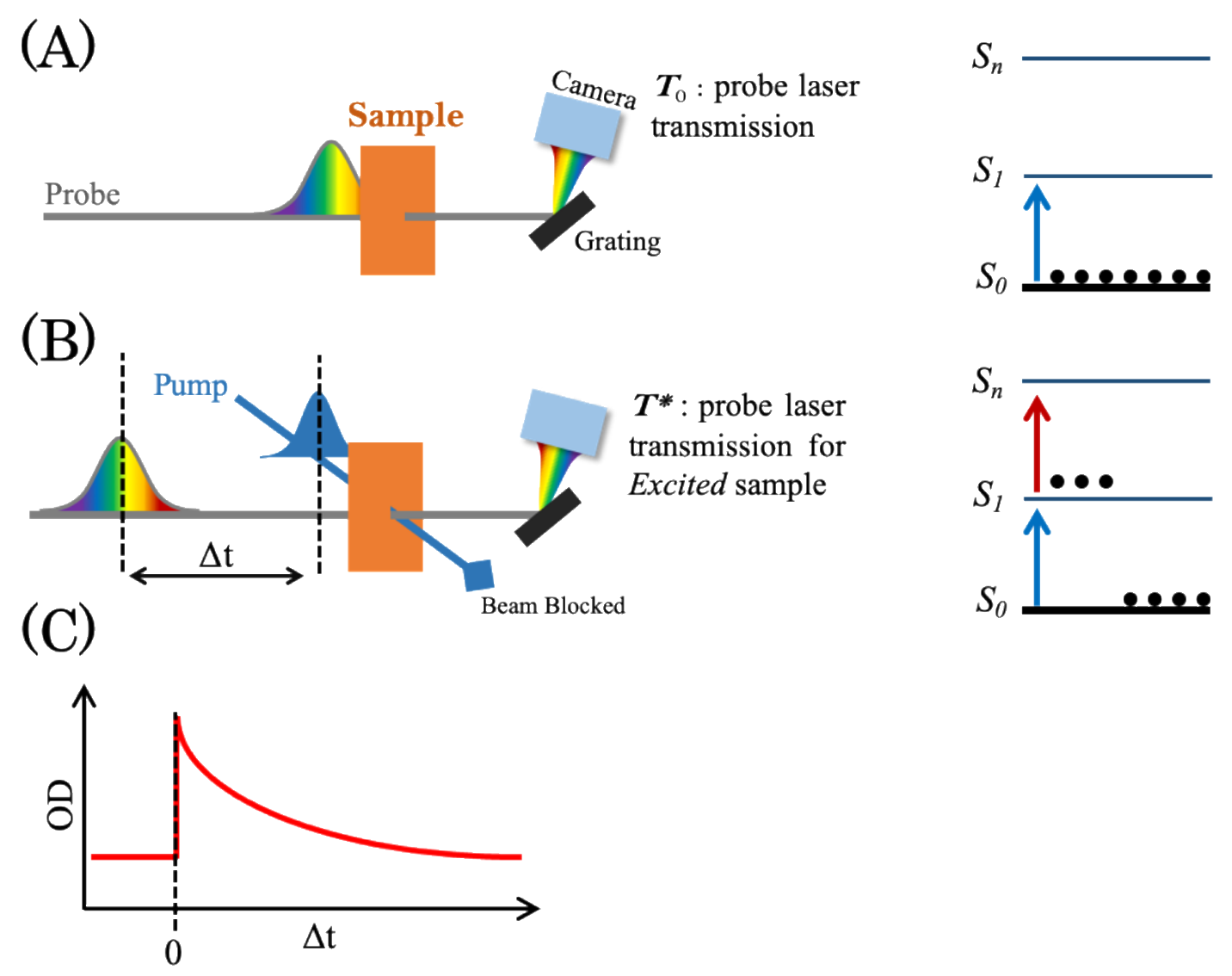

Figure 2.1. (A-left) steady state absorption spectroscopy principle left, (Aright) is an illustration of the transition monitored by probe pulse. (B-left) pump-probe TA spectroscopy with delay between the two pulses, (B-right) both populations of ground and excited states are monitored by the probe pulse. (C) introducing the pump-probe delay allows to measure the dynamics related to the excited state after subtracting of those for the ground state.

If the pump and probe pulses reach the sample at the same time, i.e., $\Delta \mathrm{t}$ pump-probe $=0$, the time is called time zero, and the excited state population monitored by the probe is usually at the maximum. A delay must be introduced between the pump and the probe, to measure the temporal evolution of the populations (signals), i.e., $\Delta \mathrm{t}_{\text {pump-probe }}>0$. The delay time between pump and probe is introduced by a motorized (mechanical) delay stage, and then the 
obtained signal intensity is proportional to the delay time (delay time represents the lifetime of the state); see figure $2.1 \mathrm{C}$. The temporal resolution is only limited by the duration time of the original light source and does not rely on the electronic response time of the detector. The delay time between the pump and the probe pulses is a result of a difference in the travel distance of the two pulses introduced by the delay stage. It is calculated and recorded as $\Delta \mathrm{t}$.

The measured transmission, in this case, corresponds to all populated states, i.e., Ground and exacted states. Thus, pumped and unpumped probe transmissions are recorded, to subtract the background transmission. This is achieved by using a synchronized optical chopper revolving at half of the repetition rate of the laser source to modulate the pump beam so that half of the pump pulses are blocked. When the pump beam is blocked, only the probe beam passes through the sample, and the transmitted signal was used as the reference signal $\left(\mathrm{I}_{0}\right)$. With the pump pulse blocked and unblocked, the changes in the optical density $(\Delta \mathrm{OD})$ are measured and recorded as a function of $\Delta \mathrm{t}$.

$$
\Delta O D\left(\Delta t, \lambda_{\text {probe }}\right)=-\log \left[\frac{I^{*}\left(\Delta t, \lambda_{\text {probe }}\right)}{I_{0}\left(\lambda_{\text {probe }}\right)}\right]
$$

where $\mathrm{I}_{0}$ and $\mathrm{I}^{*}$ are the probe beam transmission with the pump beam blocked and unblocked, respectively. 


\subsubsection{Signal possible contributions.}

Based on Beer's law, the transient absorption of the probe light is related to the population in the ground state and the excited states of the system. Therefore, different TA signals can be obtained and used to study the system under excitation. Ground state bleaching, excited states absorption, and stimulated emission are the most common TA signals. Figure 2.2A illustrates the possible transition.

After the system got excited from the ground state $\left(\mathrm{S}_{0}\right)$ to a higher electronic state $\left(\mathrm{S}_{1}\right)$ by a pump beam, the ground state is depopulated, and the ground-state absorption decreases. Hence, $\mathrm{I}^{*}$ is larger than $\mathrm{I}_{0}$, resulting in a negative $\triangle \mathrm{OD}$ signal, which is named the ground-state bleaching signal. Meanwhile, electrons that are populating the excited state lead to an excitedstate absorption. In this case, $\mathrm{I}^{*}$ is smaller than $\mathrm{I}_{0}$, resulting in a positive $\Delta \mathrm{OD}$ signal. While electrons relaxing back to the ground state via fluorescence or stimulated emission, another negative $\triangle \mathrm{OD}$ signal will be observed and is called the stimulated emission signal. The overall observed signal is the superposition of all possible signals, see figure 2.2B. 
(A)

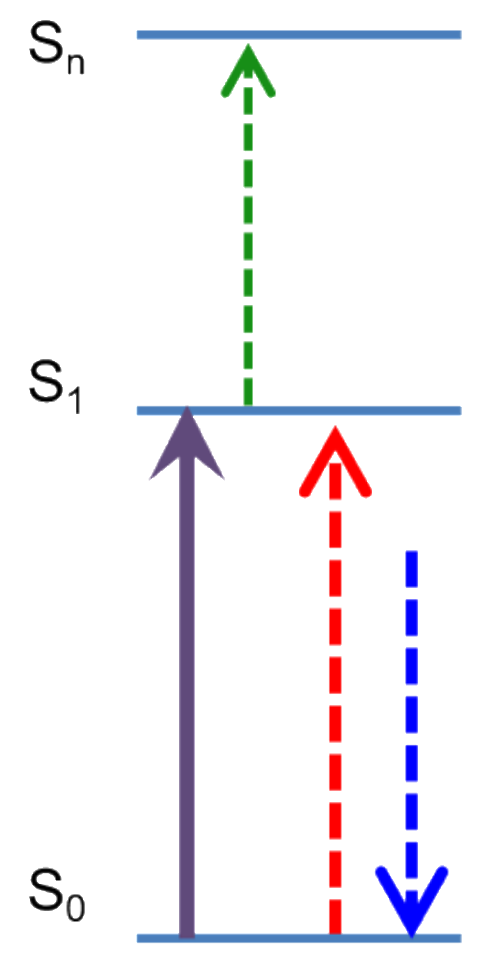

(B)

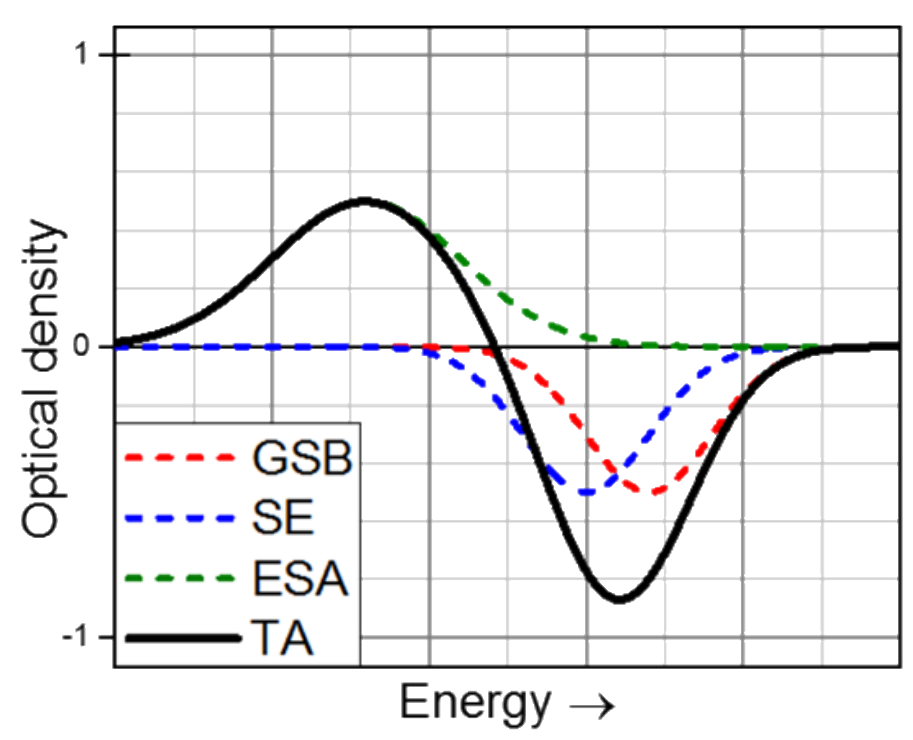

Figure 2.2. (A) possible transition upon introducing a probe pulse after a pump pulse. Violet arrow represents the pump, while dashed red arrow represents the ground state bleaching (GSB) signal and the blue is the stimulated emission (SE), the green arrow represents the excited state absorption (ESA). To the right (B) are the three signals corresponds to the three possible transitions in the left. Red is the GSB, blue is the SE, green is the ESA and the black solid line is the overall TA signal.

\subsubsection{Light source}

Ultrashort laser pulses have very high peak power. If amplified, the high peak power might burn laser gain media and optics. Thus, the peak power has to be reduced first then amplified, this is accomplished by stretching the pulses over time, so the peak power becomes significantly lower than it can be 
amplified and compressed in time. This process called Chirped Pulse Amplification (CPA).

\subsubsection{Laser head}

The femtosecond laser pulses are generated by the Clark-MXR CPA Series laser head. The laser consists of two levels. At the bottom level, a diode laser and a single-mode Erbium-doped fiber (SErF) oscillator are located, the diode laser is with an output wavelength of $980 \mathrm{~nm}$ and a power of $\sim 200 \mathrm{~mW}$. The SErF fiber laser is pumped by the diode laser. The output of the SErF fiber oscillator has a wavelength of $775 \mathrm{~nm}$ and a pulse energy of less than $1 \mathrm{~nJ}$.

At the top level, a pulse stretcher, a frequency-doubled Nd:YAG pump laser, a Ti:Sa regenerative amplifier, and a pulse compressor are located. All together represents the CPA that amplifies the bottom level output.

First, the output pulse of $\sim 100 \mathrm{fs}$ is stretched in time 200 times, i.e., to $\sim 20$ ps by a grating pair. Then, the stretched pulses are fed as the seed into the regenerative amplifier (RGA) cavity on the top level. The Ti:Sapphire regenerative amplifier is pumped by a Q-switched frequency-doubled Nd:YAG laser. After the injection of the seed into the Ti:Sa crystal. The intensity of the pulse increases every round trip inside the cavity. After several round trips in the cavity, the pulse energy reaches saturation. Therefore, the pulse exits the cavity at saturation (pulse energy $\sim 1 \mathrm{~W}$ at $1 \mathrm{kHz}$ repetition rate), and then compressed to $\sim 150 \mathrm{fs}$ pulse duration time by a pair of gratings in the pulse 
compressor. The amplified and temporally compressed pulses exit the CPA laser head and are used as the fundamental for further frequency conversions.

\subsubsection{Wavelength Tuning (frequency conversions)}

Since the fundamental output is a fixed frequency $(775 \mathrm{~nm})$, further frequency conversion methods are employed.

In the current experiment, nonlinear optical parametric amplification (NOPA), second harmonic generation (SHG), and white light generation (WL) are employed to the fundamental output. Linear optical processes are the processes where the input laser frequency is maintained in the output. While in nonlinear processes, frequency is changed, i.e., different frequencies of the input and the output. NOPA and SHG were used as pump sources, while the generated white light serves as a probe.

\subsection{Pump sources}

None linear processes include $\mathrm{n}^{\text {th }}$ harmonic generation, where $\mathrm{n}$ photons of a certain wavelength $(\lambda)$ are absorbed by a media, and emitted wavelength from that media would be equal to $(\lambda / n)$. Second-harmonics (SHG) of the fundamental output was used as one of the pump sources in this experiment; wavelength was about $388 \mathrm{~nm}$.

The second pump source in our experiment was a $490 \mathrm{~nm}$ laser pulses, generated from via NOPA box in our experimental setup. In NOPA, frequency 
is generated by pump and seed lasers overlapped spatially and temporally in a barium borate (BBO) crystal. The angle between the pump and the optical axis of the crystal is the phase-matching angle. When the SHG of the fundamental $(388 \mathrm{~nm})$ is used as the pump source, supercontinuum "white light" is used as the seed source, and phase matching angle can be tuned, the amplified signal can be obtained at the desired wavelength in the range of (460 $-680 \mathrm{~nm}$ ). NOPA output was optimized to $\sim 490 \mathrm{~nm}$ in the current experiment.

\subsection{Probe source}

When a strong, ultrafast laser pulse passes a medium, several nonlinear processes occur. Collectively, these processes cause a series of spectral broadening of the original pulse, which results in a broadband pulse, ranging from UV to near IR. The resulting laser called the supercontinuum white light source. ${ }^{81}$ In our experiment, a portion of the fundamental of the CPA at 775 $\mathrm{nm} \sim 25 \mathrm{~mW}$ was focused on BBO crystal to generate stable white light of 400 $-700 \mathrm{~nm}$ used as a probe in our experiment.

\subsubsection{Experimental setup}

Four main component boxes in the femtosecond laser system used in this experiment: CPA laser head, NOPA, SHG, and Transient absorption PumpProbe Spectrometer (TAPPS).

As shown in Figure 2.3, the CPA laser head generates a fundamental output (Red arrows). The output is split into the NOPA and the SHG (Blue 
arrows). In the NOPA, the fundamental goes through two stages of amplification and a pulse compressor. The Output of the NOPA was used as a pump source (Green arrow). The supercontinuum white light is generated in the TAPPS Box and used as a probe (Rainbow colored arrows).

In pump-probe, the pump and the probe beams go through different beam paths, and each path involves a retro-reflector. The retro-reflector for the probe beam is on a rail track can be adjusted manually. The retro-reflector of the pump beam is fixed on a motorized translation stage by the data acquisition program. Therefore, the delay time between the pump and probe can be calculated from the difference in the length of the two beam paths, which in turn is determined by the software. 


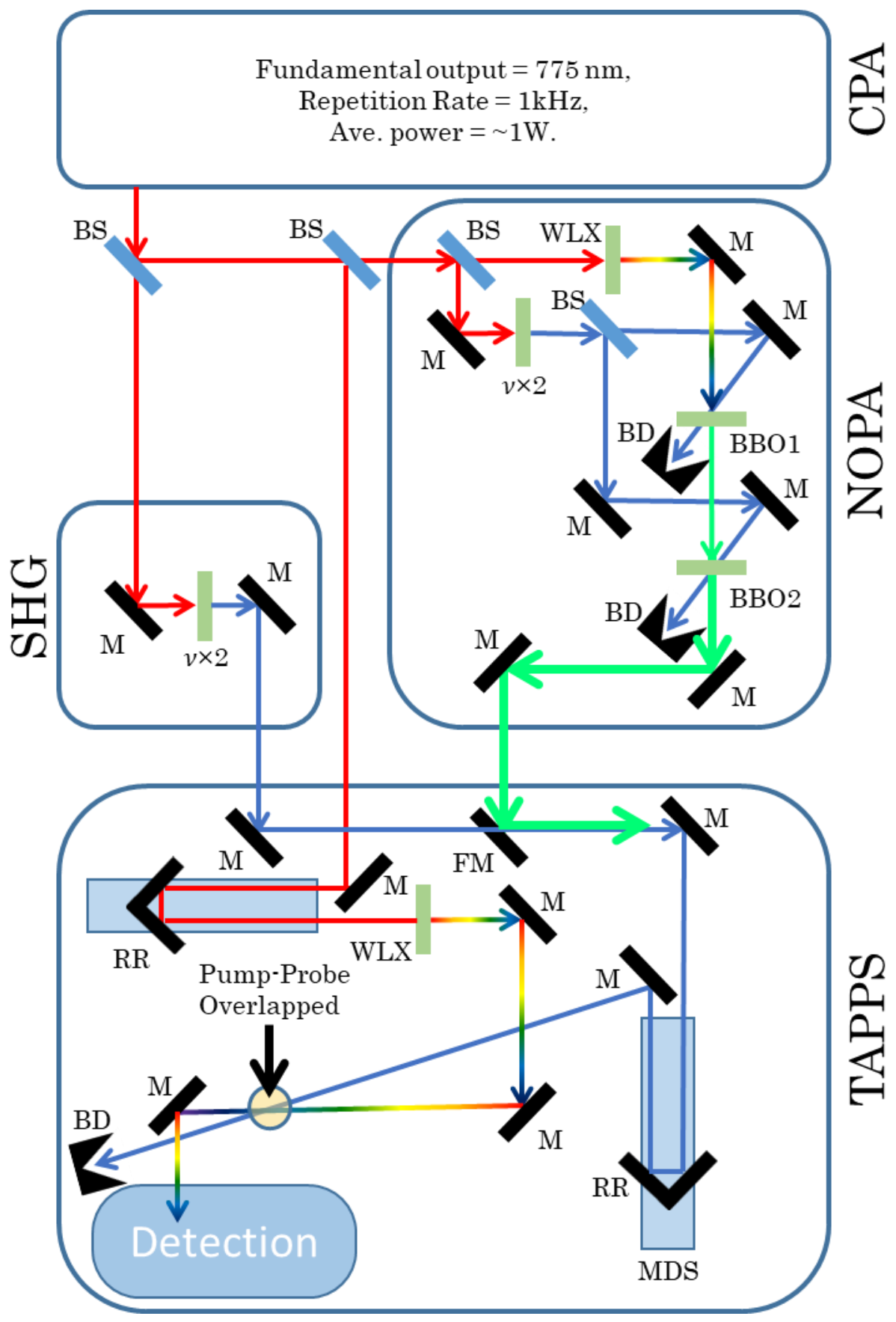

Figure 2.3. A schematic illustrates our experimental layout. CPA, NOPA, SHG and TAPPS are shown in the figure. Red arrows represent the fundamental, blue is the SHG output, green is the NOPA output and the rainbow colored is whit light. M: mirror, BS: beam splitter, WLX: white light crystal, BBO: NOPA crystal, BD: beam dumber, $v \times 2$ : SHG crystal, FM: flipping mirror, RR: retro reflector, MDS: motorized delay stage. Some optical components like irises and filters and some others are omitted for simplicity. The pump and probe are overlapped in the sample (yellow circle). 


\subsection{Cavity ring-down Principle}

\subsubsection{Absorption Spectroscopy}

Absorption can be measured according to Beer-Lambert Law:

$$
A=\frac{I_{0}}{I}=10^{\sigma l c}
$$

where $A$ is the absorbance, $I_{0}$ is the initial light intensity, $I$ is the transmitted light intensity, $\sigma$ is the absorption cross section, $l$ is the absorption path length, and $c$ is the concentration.

When absorption is weak, two methods can be used to obtain a spectrum; increasing the concentration, or the absorption path length. However, in the case of radical target species, for example, increasing the concentration might cause side reactions. Peroxy radicals, for example, have a very low absorption cross section in the IR region $(\tilde{\mathrm{A}} \leftarrow \tilde{\mathrm{X}}$ Transition discussed in chapter 4). increasing the concentration will initiate the peroxy radicals self-reaction, which will cause the presence of other unwanted peroxy radicals, and makes the spectra complicated.

On the other hand, increasing the absorption path length is impossible for technical reasons. In this experiment, we generate our target species (peroxy radicals) using the photolysis of precursors (discussed in section 2.2.4). This also requires the photolysis to be extended along the detection axis to generate the target species. If a long reaction cell is used, the photolysis beam 
will be too long. Hence its fluence will be very low, and target radicals would not be produced in the reaction cell.

Since neither long-pass absorption spectroscopy nor increasing the radical concentrations are possible (in our experiment), special techniques must be employed to overcome such a problem. Cavity ringdown spectroscopy (CRDS)82-85 is a suitable experimental technique for the detection of small concentrations and low absorption cross section materials.

\subsubsection{CRDS principle}

In CRDS, high-reflectivity mirrors are used to make an optical cavity. Due to high reflectivity, if a laser is injected into the cavity, a small amount of light will be transmitted through the mirrors. The leaked intensity of the transmitted light is detected, and it is proportional to the intensity of light in the cavity. The overall intensity of the transmitted laser reaches the detector decays exponentially, due to the loss of the laser intensity each round trip in the cavity. This observed decay is called the ringdown curve. The bouncing of the laser light between the cavity mirrors increases the absorption pathlength (1), which improves the sensitivity of the experiment significantly without increasing concentration or cell length. Figure 2.4 illustrates the CRDS principle. 
A
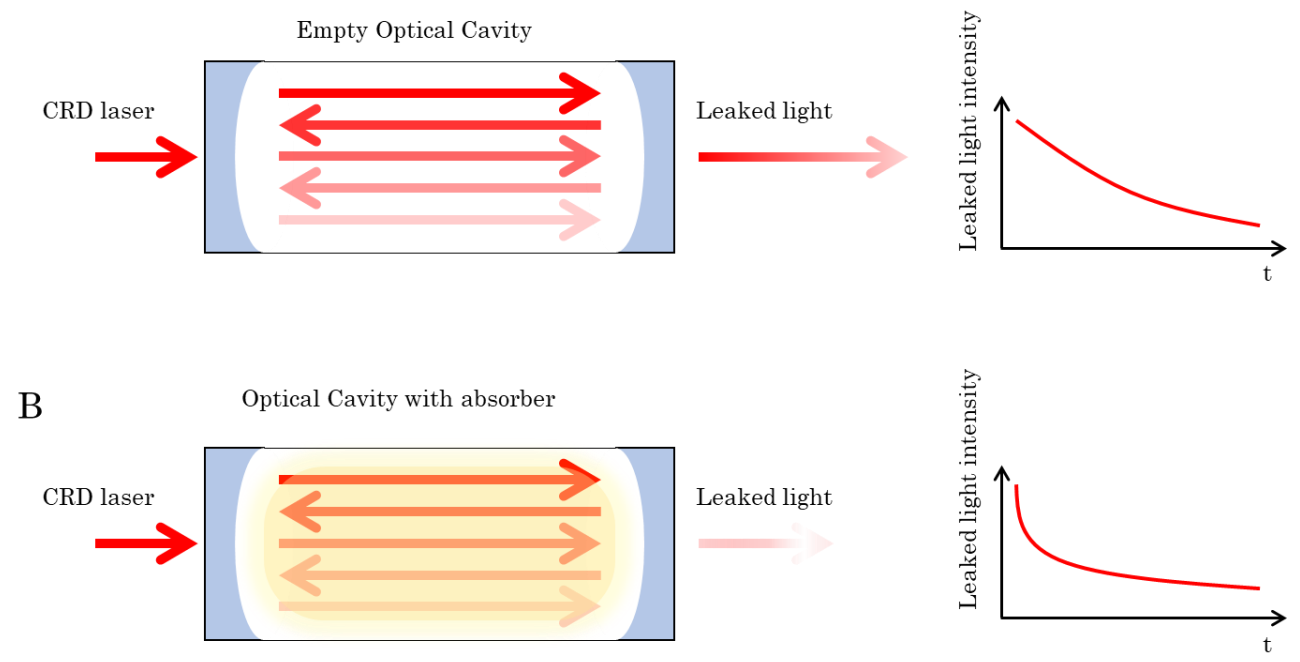

Figure 2.1. Illustration of the CRDS principle, when light is injected to an empty cavity (A-left), the laser will bounce back and forth between cavity mirrors, the leaked light intensity is recorded with time (A-right) and result in an exponential decay curve called ring down curve, exponential fitting result is the ring down time. If an absorber is introduced in the cavity (B-left), the leaked intensity decreases, hence ring down time decrease (B-Right). Absorption per pass is calculated from the difference in the ring down times.

Ideally, the ring down curve should consist of one exponential decay, indicating only one laser mode injected into the cavity. However, if pulsed lasers were used, this is usually not the case, where multi modes are injected into the cavity, and multi-exponential decay is expected.

Adapting Beer-Lambert law to calculate the loss of the intensity due to the cavity (eq.2.3),

$$
\mathrm{I}_{\text {cavity }}=\mathrm{I}_{0} \cdot \mathrm{e}^{\left[-\left(\frac{\text { Loss }}{\text { Reflection }}\right) \cdot\left(\frac{\# \text { of reflections }}{\text { Round trip }}\right) \cdot(\# \text { of Round trips })\right]}
$$

If $\mathrm{R}$ were the reflectivity of the mirrors, then, 


$$
\text { loss per reflection }=1-\mathrm{R}
$$

The number of round trips in time ( $\mathrm{t}$ ) would be given by (eq.2.5)

$$
\text { \# of Round trips }=\frac{\mathrm{t} \cdot \mathrm{c}}{2 \mathrm{~L}}
$$

Where (L) is the length of the ring-down cavity, and (c) is the speed of light.

Since the cavity consists of two mirrors, then the number of reflections per round trip is equal to two. By substituting equations 2.4 and 2.5 in equation 2.3 results in Eq. 2.6, which describes the loss of the intensity of laser light in the empty cavity.

$$
\mathrm{I}_{\text {cavity }}=\mathrm{I}_{0} \cdot \mathrm{e}^{\left[-(1-\mathrm{R}) \cdot\left(\frac{\mathrm{t} \cdot \mathrm{c}}{\mathrm{L}}\right)\right]}
$$

The amount of time it takes for the light decay is called "empty cavity ring-down" time $\left(\tau_{0}\right)$ defined as the time for the light intensity to decay to the 1/e of the initial intensity $\left(\mathrm{I}_{0}\right)$ and given by:

$$
\tau_{0}=\frac{\mathrm{L}}{\mathrm{c} .(1-\mathrm{R})}
$$

Similarly, the loss of intensity due to the absorber (sample) can be described by:

$$
\begin{gathered}
\mathrm{I}_{\text {sample }}=\mathrm{I}_{0} \cdot \mathrm{e}^{\left[-\left(\frac{\text { Loss }}{\text { Round trip }}\right) \cdot(\# \text { of Round trips })\right]} \\
\mathrm{I}_{\text {sample }}=\mathrm{I}_{0} \cdot \mathrm{e}^{\left[-(2 \sigma \mathrm{nl}) \cdot\left(\frac{\mathrm{t} \cdot \mathrm{c}}{2 \mathrm{~L}}\right)\right]}
\end{gathered}
$$


Where (l) is the effective path length. Combining equations 2.8 and 2.7 results in

$$
I=I_{0} \cdot e^{\left[-[(1-R)+(2 \sigma n l)] \cdot\left(\frac{t c c}{2 L}\right)\right]}
$$

The ring-down time of the cavity with the absorbing sample, $\tau_{a b s}$, is given by

$$
\tau_{\mathrm{abs}}=\frac{\mathrm{L}}{\mathrm{c.} \cdot(1-\mathrm{R})+\sigma \mathrm{nl}]}
$$

The quantity ( $\sigma \mathrm{nl}$ ) is the Absorption (A) of a sample, then Combining equations 2.7 and 2.10 results in

$$
\mathrm{A}=\frac{\mathrm{L}}{\mathrm{c}}\left(\frac{1}{\tau_{\mathrm{abs}}}-\frac{1}{\tau_{0}}\right)
$$

CRDS is based on measuring the decay rate of the light trapped in a high finesse optical cavity. Therefore, the ability to accurately measure the ringdown time $\tau$ will limit CRDS sensitivity. Upon introduction of a small absorption per pass, into a cavity, the empty cavity ringdown time $\tau_{\text {empty }}$ will decrease by $\Delta \tau$,

$$
\Delta \tau=\tau_{a b s}-\tau_{0}
$$

And according to equation 2.11,

$$
A=(1-R)\left(\frac{\Delta \tau}{\tau_{\mathrm{abs}}}\right)
$$


Strong absorption will result in a short ring-down time $\tau_{\mathrm{abs}}$ leading to the decrease in accuracy of the absorption measurements. Higher mirrors reflectivity $\mathrm{R}$ will result in longer ringdown times and higher sensitivity. It is also evident that sensitivity depends on relative measurement error, $\frac{\Delta \tau}{\tau}$.

The sensitivity of the pulsed CRDS apparatus can be substantially increased by ensuring a single-mode excitation in the cavity and matching laser to $\mathrm{TEM}_{00}$ mode. Cavity ring-down spectroscopy has a major advantage over traditional spectroscopic techniques. Measurement of decay rate in the time domain is intrinsically more accurate than observing small intensity changes on top of the strong laser pulse background. Therefore, unlike most pulsed, direct absorption methods, the cavity ringdown is insensitive to the fluctuation of probe pulse intensity.

For a typical flow cell CRDS experimental setup, $\mathrm{L}=\mathrm{l}=0.5 \mathrm{~m}, \mathrm{R}=$ $99.995 \%$, the empty-cavity ring-down time is $33 \mu \mathrm{s}$, and the effective path length is $10 \mathrm{~km}$. A minimum detectable absorption coefficient of $\sim 0.3 \mathrm{ppm}$ per pass can be achieved.

\subsubsection{CRDS Cavity alignment}

A will aligned ringdown cavity is necessary for the maximum ring-down curve, which leads to better signal to noise ratio. Since the invisible IR beam is going to be injected into the cavity, cavity alignment faces lots of difficulties. A colinear HeNe laser beam used to align the cavity. The first step is to ensure 
that the HeNe laser is overlapped with the IR beam just before the cavity. This is done by directing the visible fundamental exiting out of the Raman shifter cell into two irises using a set of two mirrors; this will automatically align the IR beam. Then before the set of mirrors and the irises, the HeNe laser beam is centered on the same irises using a different set of mirrors. It is very important not to touch the mirrors used in the IR alignment.

The HeNe is then directed through the cell, using the optics and mirrors after the two irises, aligned onto the center of both ends. Although CRD mirrors are transparent to visible light, there will be a reflection of the $\mathrm{HeNe}$ from both surfaces of the mirror. The brighter spot corresponds to the inside surface of the mirror, which is the important surface for the cavity alignment. This reflection spots of the two mirrors should be aligned to overlap with the original HeNe beam. This is done by centering the back reflection onto a white card with a pinhole smaller or equal to the diameter of the beam. After finishing the alignment, the exit beam of the cavity must be aligned to the detector to observe the ringdown curve and fine-tune the alignment. The detector should be connected to an oscilloscope. A detailed, step by step procedure is explained in appendix A.

\subsubsection{Radical generation via photolysis}

Two main methods can be used to produce the peroxy radicals via photolysis, first; Direct photolysis of the halogenated precursor, where the 
halogen is at the target position of the radical, in the presence of oxygen and nitrogen buffer gas. ${ }^{86}$ The photolysis wavelength (photon energy) is chosen based on the halogen-carbon bond energy. The criteria for choosing a photolysis wavelength depend on the experiment itself, such as the absorption cross section of the precursors and halogen substitution on the precursors. Target radicals are formed according to the following chemical equations:

$$
\begin{gathered}
\mathrm{R}-\mathrm{X} \stackrel{\mathrm{h} v(\lambda=266 \text { or } 193 \mathrm{~nm})}{\longrightarrow} \mathrm{R} \bullet+\mathrm{X} \bullet \\
\mathrm{R} \bullet+\mathrm{O}_{2} \rightarrow \mathrm{ROO} \bullet
\end{gathered}
$$

Nitrogen gas usually used as a carrier gas and acts as a third body in the reaction.

Second; radicals are produced via hydrogen abstraction by chlorine attack, ${ }^{87-88}$ produced in $193 \mathrm{~nm}$ photolysis of oxalyl chloride $(\mathrm{COCl})_{2}$ :

$$
\begin{gathered}
(\mathrm{COCl})_{2} \stackrel{\mathrm{hv}(\lambda=266 \text { or } 193 \mathrm{~nm})}{\longrightarrow} 2 \mathrm{CO}+\mathrm{Cl} \bullet \\
\mathrm{Cl} \bullet+\mathrm{R}-\mathrm{H} \rightarrow \mathrm{R} \bullet+\mathrm{HCl} \\
\mathrm{R} \bullet+\mathrm{O}_{2} \rightarrow \mathrm{ROO} \bullet
\end{gathered}
$$

\subsubsection{Light sources}

\subsubsection{Photolysis lasers}

In this experiment, two different lasers were used for photolysis. 


\section{ArF Excimer Laser}

ArF excimer laser (Lambda Physik, LPX 120i) was used to provide the $193 \mathrm{~nm}$ photons for photolysis. The laser is controlled via pc with a fiber optics connection.

First, the laser has to be filled with the correct gas ratio to obtain the max output. If necessary, the laser can be passivated with $\mathrm{F}_{2}$ gas and helium burned, to make sure the tube is clean and saturated with $\mathrm{F}_{2}$ gas. Otherwise, laser power could decrease rapidly. The complete procedure of the passivation, the hilum burn, gas filling are described in detail in appendix A.

The correct ratios for gas mixture to be filled in the laser head are summarized in table 2.1 .

Table 2.1. Gas mixture ratios for ArF excimer laser, output $193 \mathrm{~nm}$.

\begin{tabular}{ccc} 
Gas & Amount (mbar) & Total (mbar) \\
\hline$\% 5 \mathrm{~F}_{2}$ in He Balance & 130 & 130 \\
\hline $99.999 \% \mathrm{Ar}$ & 250 & 380 \\
\hline $99.999 \% \mathrm{Ne}$ & 2620 & 3000 \\
\hline \hline
\end{tabular}

After filling the excimer laser, the power was measured directly at the exit aperture and found to be $250 \mathrm{~mJ}$. The spot is a rectangular shape with 1 $\times 3 \mathrm{~cm}^{2}$ dimensions. Due to the absorption of the atmospheric oxygen and generation of ozone gas, the laser cannot travel freely in the space, it has to be 
contained, where ozone generation prevented for health and safety, a vacuumed tube with two glass windows along the whole travel distance was prepared to prevent ozone generation in the atmosphere of the lab. On the other hand, the output power of the laser will decrease drastically after about 1-meter travel distance due to the absorption. The vacuumed tube also helped in maintaining the power of the laser.

The laser then directed to the absorption cell and re-shaped to a rectangular shape of $12 \times 0.5 \mathrm{~cm}^{2}$ by using cylindrical diversion lens, where the curvature axis was parallel to the laser spot in order to expand the beam in the horizontal axis, then it was followed by another cylindrical conversion lens perpendicular to the first lens to stretch the beam on the vertical axis. This increases the photolysis efficiency and the absorption length of the produced radicals. After reshaping the beam, the fluence of the $193 \mathrm{~nm}$ photolysis laser was $\sim 15 \mathrm{~mJ} / \mathrm{cm}^{2}$.

\section{Nd:YAG fourth harmonic}

Also, the fourth harmonic of an Nd:YAG laser (Spectra-Physics, QuantaRay Pro-270, $\lambda=266 \mathrm{~nm}$ ) was used as the photolysis source. Similar to the excimer laser mentioned above, a set of diverging and converging cylindrical lenses was used to reshape the photolysis-laser beam cross section to a $12 \times 0.5$ $\mathrm{cm}^{2}$ rectangle. Fluences of both 266 and $193 \mathrm{~nm}$ photolysis lasers were similar for comparison in control experiments $\sim 15 \mathrm{~mJ} / \mathrm{cm}^{2}$. 


\subsubsection{CRD Laser}

The laser light used in probing our target species was generated by several consecutive processes. First, the second harmonic of an Nd:YAG laser (Spectra-Physics, Quanta-Ray Pro-270) was used to pump a tunable dye laser (Sirah, PrecisionScan). Then the output of the dye laser was focused at the center of an $\mathrm{H}_{2}$ cell to generate near-IR radiation by Raman shifting. Figure 2.5 shows a Schematic for the CRD laser source used in the current experiment.

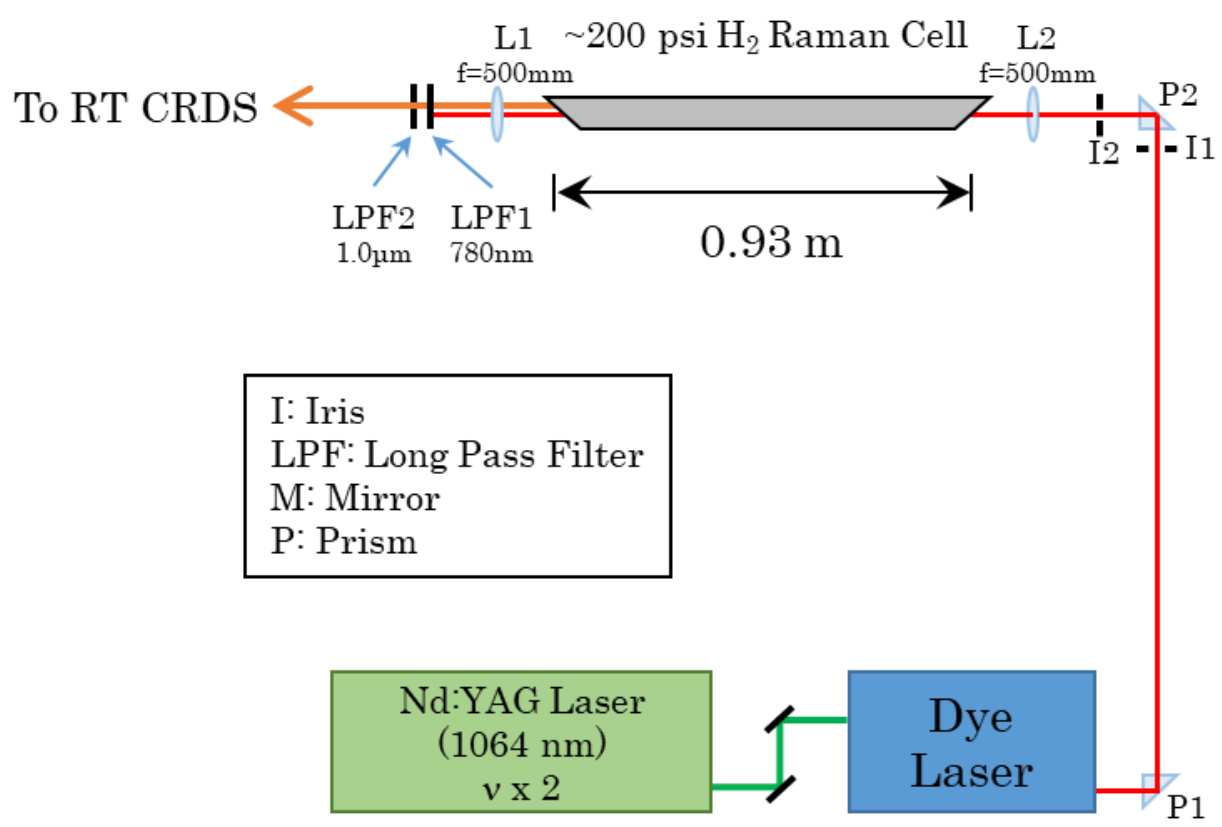

Figure 2.5. A schematic illustrates the CRD light source used, the dye laser is pumped by second harmonics of the Nd:YAG laser then focused on the hydrogen Raman cell to generate IR laser.

The pyrromethene and DCM dyes were used to cover the wavelength range of $\sim 585$ to $\sim 670 \mathrm{~nm}$. The visible output from the dye laser with pulse energy $\sim 90 \mathrm{~mJ}$ was directed to Raman shifter cell to generate a laser beam in 
the wavelength range of $\lambda=1.15-1.55 \mu \mathrm{m}$. The fundamental, the anti-Stokes, and the first Stokes radiations were blocked by two long-pass filters (cutoffs = 780 and $1000 \mathrm{~nm}$ ). The transmitted second Stokes radiation was collimated by a telescope consisting of two confocal lenses $(f=7.5$ and $2.5 \mathrm{~cm})$. A $100 \mu \mathrm{m}$ diameter pinhole was placed at the focal point for mode selection. The resulting second Stokes radiation (1-2 mJ) was directed through the reaction cell.

The linewidth of the visible fundamental from the dye-laser measured $0.06 \mathrm{~cm}^{-1}$ using a spectrometer (HighFinesse, WS-7). The linewidth of the nearIR radiation is limited by the pressure and Doppler broadenings in the $\mathrm{H}_{2}$ cell to about $1 \mathrm{GHz} .{ }^{89-90}$ Figure 2.6 is a schematic that shows our laser sources along with the reaction cell. 


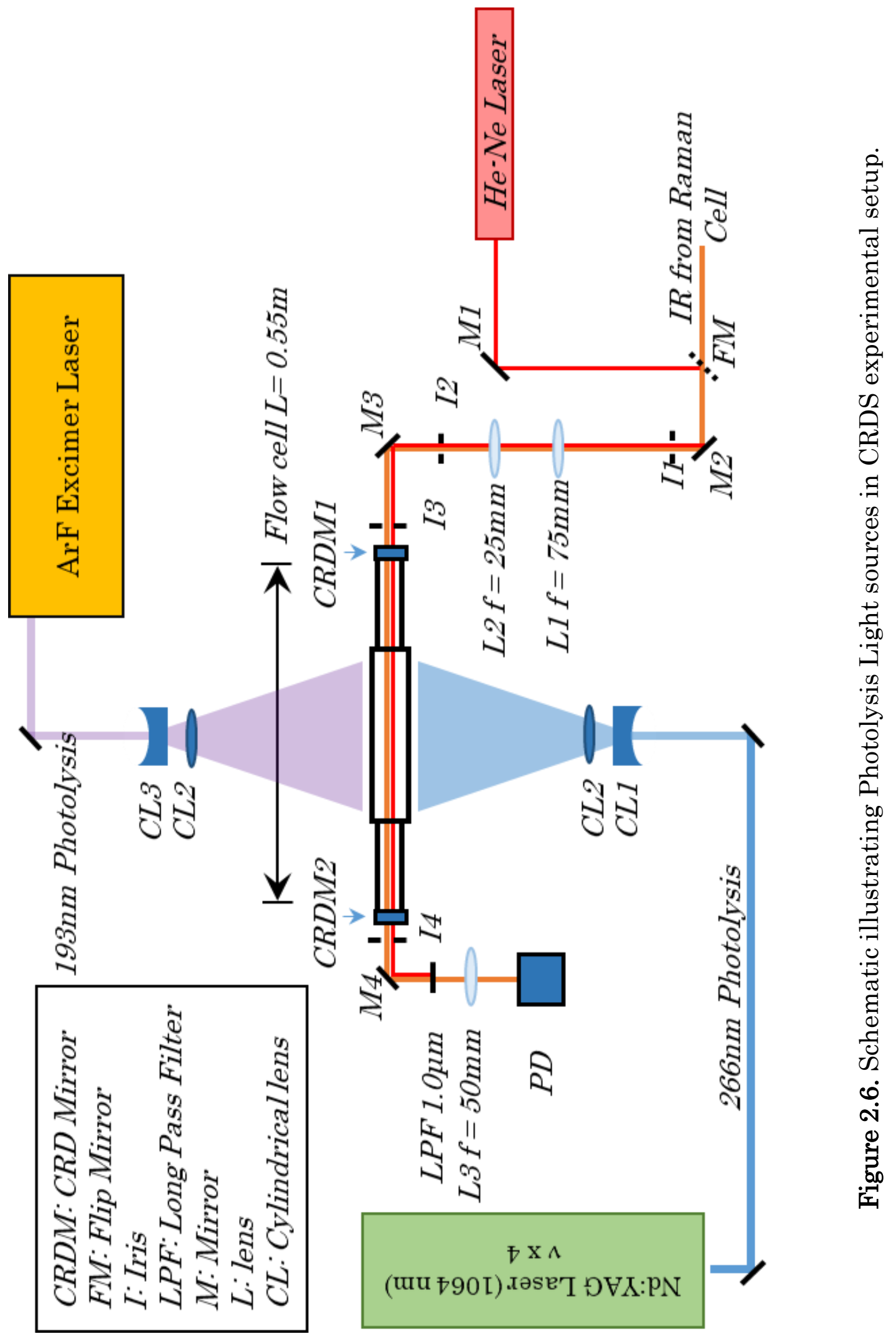




\section{Raman Shifting cell}

Raman effect on materials will cause frequency conversion of the incident light from one wavelength to another. When light enters a medium, the molecules of that medium can be excited to its excited states. When the excited molecules relax back to their ground state, they can emit light of higher frequency (anti-Stokes), the same frequency (Rayleigh), or lower frequency (Stokes). The frequency shift between the incident and shifted photons is dependent on the medium used. Hydrogen gas has a frequency shift of 4155.2 $\mathrm{cm}^{-1}$. Therefore, the stock shifted generated from our fundamental will cover the range of $1.15-1.55 \mu \mathrm{m}$. Figure 2.7 illustrates the Raman shifting process for hydrogen.

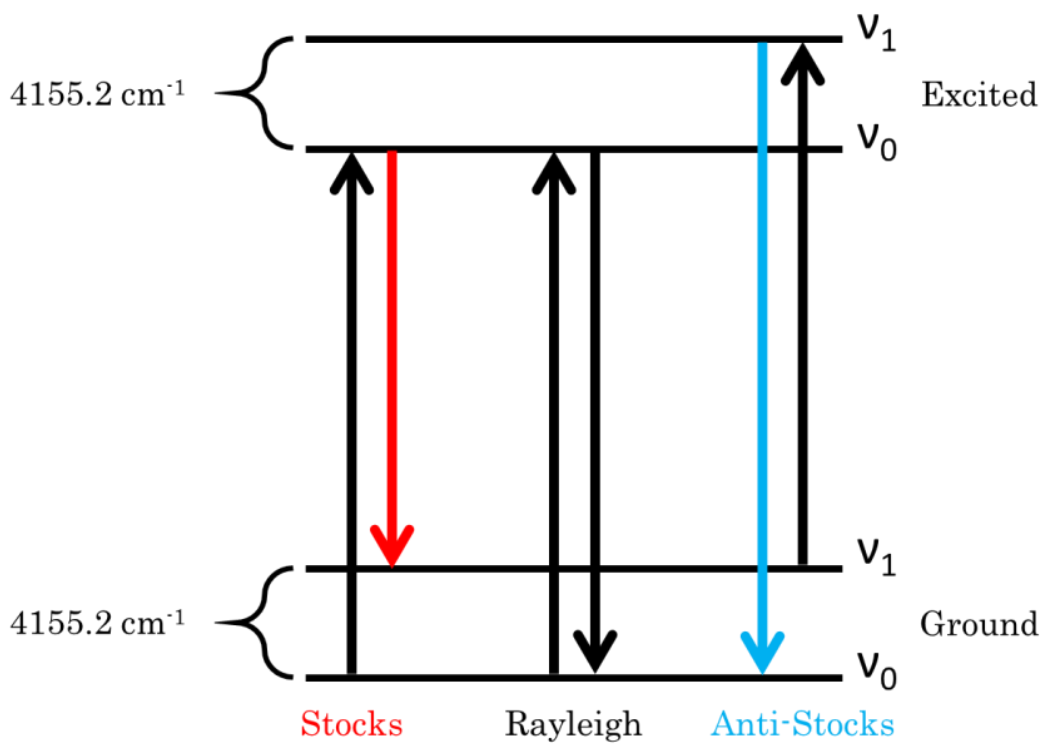

Figure 2.7. Energy diagram illustrating the Raman effect. If molecules were excited, it relaxes back to the ground state with lower frequency (Stokes), or with the same frequency (Rayleigh), or of higher frequency than the input (anti-Stokes). 


\subsubsection{Room-Temperature reaction cell}

A $20 \mathrm{~cm}$ stainless-steel reaction cell was used in the current experiment..,91 The cell has two rectangular UV-grade quartz side windows for the photolysis laser beam. CRD mirrors (Los Gatos Research, $R>99.99 \%$ ) was mounted on commercially available mounts, mounted on a $15 \mathrm{~cm}$ long arms on both sides. The arms are attached to the cell body to form a ring-down cavity.

The reaction cell was connected to a vacuum pump with a needle valve to control the pumping speed and total pressure in the reaction cell. CRD mirrors and quartz windows were purged by $\mathrm{N}_{2}$ flow continuously to prevent contamination. Figure 2.8 illustrates the reaction cell, along with the gas delivery system.

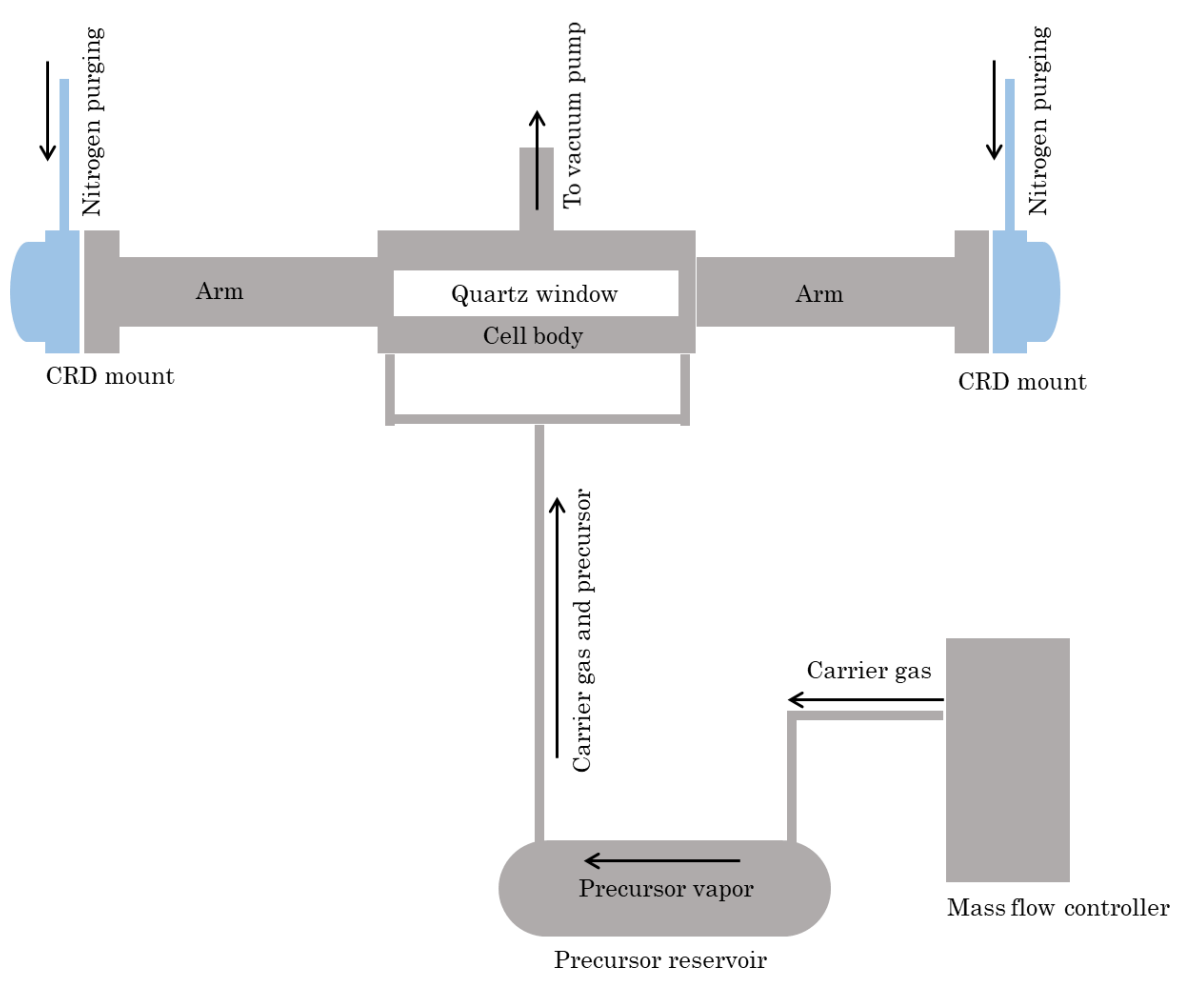

Figure 2.8. Reaction cell setup and gas flow diagram. 
Precursors were stored in stainless steel reservoirs. The flow of the carrier above the liquid samples and carried their vapors into the stainlesssteel reaction cell. Saturation vapor pressures were controlled and stabilized by immersing precursors reservoirs in a 1:1 water-ethylene glycol bath $\left(-10^{\circ} \mathrm{C}\right)$. A gas delivery system with mass flow controllers (MKS, 247D and GE50A) was used to control the flow rates by controlling the flow of the carrier gases.

\subsubsection{Detection, Timing control, and delay generation}

The Transmitted CRD laser through the cavity was focused on and detected by an InGaAs amplified photodiode (Thorlabs, PDA10CS). The signal was acquired with an oscilloscope (Tektronix, DPO3014). The ring-down curves were averaged 32 times at each laser wavelength before being sent to a $\mathrm{PC}$ via a USB port. A home-built LabVIEW program was used for data processing. The averaged ring-down curves of up to 100,000 data points were fit to a biexponential decay function. The longer of the two fit time constants was used to calculate the fractional absorption (in ppm/pass). If necessary, ring-down curves sent to the $\mathrm{PC}$ or determined ring-down times at each wavelength were further averaged by the LabVIEW program.

CRD laser, Photolysis laser, and the oscilloscope were synchronized using a delay generator (Stanford Research System, DG645) at a $20 \mathrm{~Hz}$ repetition rate. The LabVIEW program controlled the on/off of the excimer laser and wavelength scan of the dye laser buy sending a TTL trigger pulse to 
an and gate, that combines the PC TTL pulse and the trigger of the delay generator. Sirah laser was running on a triggered scan mode, Sirah wavelength change was triggered at the falling edge of the PC TTL pulse each off event. Figure 2.9 shows the timing control sequence of all instruments. In the figure, (A) represents the TTL out of the PC generated by the LabVIEW program, (B) is the TTL generated by the delay generator, sent to both Nd:YAG and oscilloscope, (C) TTL Out of the and gate, sent to the excimer laser. The output of the and gate is generated by adding the TTL signals from A and B, and (D) represent the wavelength change event of the dye laser triggered at the falling edge of the TTL pulses on trace A. each laser pulse on trace B represents a ring-down event. The time separation of each laser pulse is determined by the repetition rate, while the TTL pulse duration generated from the PC (trace A in figure 2.9) is determined by the averaging of the ring down traces multiplied by the repetition rate. The Sirah laser will change the wavelength at the falling edge of the TTL signal generated on trace A and will park at that wavelength until all measurements are done. 


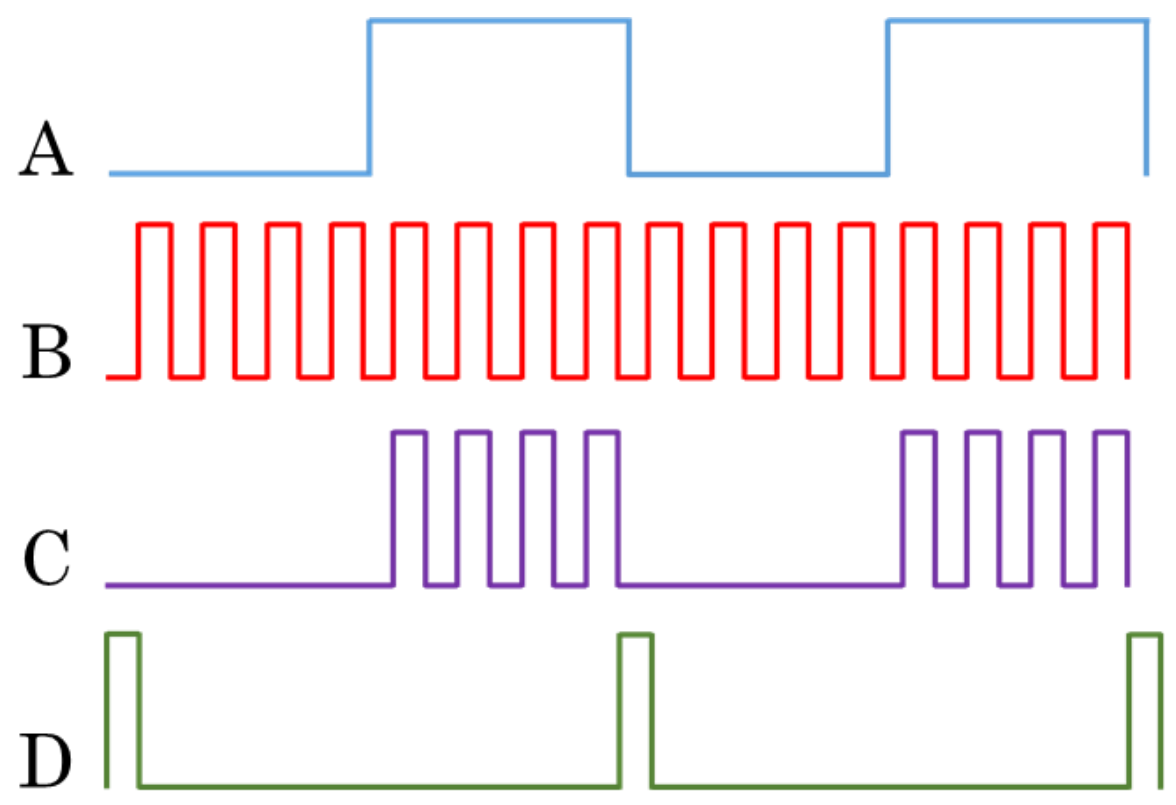

Figure 2.9. Timing control and synchronization of all instruments in the current experiment. (A) represent the TTL out of the PC generated by the LabVIEW program, (B) is the TTL generated by the delay generator, sent to both Nd:YAG and oscilloscope, (C) TTL Out of the and gate, sent to the excimer laser. The output of the and gate is generated adding the TTL signals from A and B, and (D) represent the wavelength change event of the dye laser triggered at the falling edge of the TTL pulses on trace A. 
CHAPTER 3

ULTRAFAST EXCITON DYNAMICS IN SHAPE-

CONTROLLED METHYLAMMONIUM LEAD

BROMIDE PEROVSKITE NANOSTRUCTURES:

\section{EFFECT OF QUANTUM CONFINEMENT ON CHARGE CARRIER RECOMBINATION}

\subsection{Introduction}

It is well known that the morphology of photovoltaic materials affects both the steady-state and time-resolved spectra significantly. Especially, the electron-hole interaction and local charge carrier lifetimes in perovskite thin films are impacted by their microstructure. ${ }^{92-93}$ The quantum confinement effect is manifested in both the UV-visible absorption and the photoluminescence (PL) spectra of perovskite nanostructures.

Although thin-film perovskites have been extensively studied by TA and other ultrafast spectroscopic techniques, ${ }^{42}$ charge carrier dynamics in lowerdimensional perovskites, especially one-dimensional nanowires and nanorods, were not investigated in a great detail. ${ }^{94-102}$ 
In this chapter, femtosecond TA spectroscopy studies on the photoinduced dynamics of a series of methylammonium lead bromide $\left(\mathrm{CH}_{3} \mathrm{NH}_{3} \mathrm{PbBr}_{3}\right)$ nanostructures: nanocrystals (NCs, 0D), nanowires (NWs, 1D), and nanoplatelets (NPs, 2D). The exciton Bohr radius ( $\mathrm{a}_{\mathrm{B}}$ ) of $\mathrm{CH}_{3} \mathrm{NH}_{3} \mathrm{PbBr}_{3}$ perovskite was calculated to be $1.36 \mathrm{~nm},{ }^{103-104}$ using the effective masses of electrons and holes, comparable or smaller than the dimensions of the nanostructures. Our perovskite nanostructures are therefore not in the strong confinement regime, in which the electron and hole exist predominantly as separate particles rather than excitons. ${ }^{105}$

Information on photoinduced dynamics has been derived from the analysis of the TA spectra. Specifically, sub-picosecond hot electron and hole cooling has been observed, while the ensuing recombination process is on timescales of tens or hundreds of picoseconds depending on the initial charge carrier density. Time evolution of the state-filling, stimulated emission, and intraband absorption signals in the TA spectra are governed by these two processes, while bandgap renormalization induces a sub-picosecond sub-bandgap absorption signal. Comparison between fit values of peak centers and time constants of various photoinduced processes in all three nanostructures under different pump fluences, especially those of charge carrier recombination, demonstrates that structural and dimensional differences affect not only band structure but also charge carrier dynamics. 


\subsection{Experiment}

\section{Chemicals}

Lead(II) bromide $\left(\mathrm{PbBr}_{2}, 99.999 \%\right)$, methylamine solution $\left(\mathrm{CH}_{3} \mathrm{NH}_{2}, 2.0\right.$ $\mathrm{M}$ in THF), hydrobromic acid ( $\mathrm{HBr}, 48 \%)$, n-octylamine $\left(\mathrm{CH}_{3}\left(\mathrm{CH}_{2}\right)_{7} \mathrm{NH}_{2}, 99 \%\right)$, oleic acid (99\%), octanoic acid (99\%), 1-octadecene (1-ODE, 90\%), acetone (99.5\%, ACS reagent) and toluene (99.5\%, ACS reagent) were purchased from Sigma-Aldrich. N,N-dimethylformamide (DMF) was purchased from Fischer Scientific. Before use ODE was kept under a high vacuum overnight and transferred to the glovebox under $\mathrm{N}_{2}$ atmosphere. All solvents were purged with $\mathrm{N}_{2}$ for 30 min before use.

\section{Methylammonium and octyl ammonium bromide synthesis}

Bromide salts of methylamine and n-octylamine were prepared using a modified literature procedure. ${ }^{49,106}$

$\mathrm{HBr}(10.84 \mathrm{~mL}, 0.2 \mathrm{~mol})$ was added drop-wise to a solution of $\mathrm{CH}_{3} \mathrm{NH}_{2}$ (7.2 mL, $0.2 \mathrm{~mol})$ or $\mathrm{CH}_{3}\left(\mathrm{CH}_{2}\right)_{7} \mathrm{NH}_{2}(32.98 \mathrm{~mL}, 0.2 \mathrm{~mol})$ and ethanol $(20 \mathrm{~mL})$ at $0{ }^{\circ} \mathrm{C}$ under stirring. The stirring continued for $3 \mathrm{hrs}$ before the ethanol was removed by rotary evaporation. The solid was again dissolved in a minimum amount of ethanol and recrystallized by the addition of ether. The solid was then dried under vacuum, resulting in a snow-white crystalline material that was stored inside the glove box for further use. 


\section{NCS Synthesis}

In $\mathrm{CH}_{3} \mathrm{NH}_{3} \mathrm{PbBr}_{3} \mathrm{NCs}$ synthesis, a $120 \mu \mathrm{L}$ of oleic acid $(0.31 \mathrm{mmol})$ was dissolved in $2-\mathrm{mL}$ of $1-\mathrm{ODE}$ in a two-neck round bottom flask under $\mathrm{N}_{2}$. At 90 ${ }^{\circ} \mathrm{C}$, the reaction mixture was stirred for $45 \mathrm{~min}$. At this point, $0.0126 \mathrm{~g}(0.06$ mmol) of solid $\mathrm{CH}_{3}\left(\mathrm{CH}_{2}\right)_{6} \mathrm{CH}_{2} \mathrm{NH}_{3} \mathrm{Br}$ was added to the reaction mixture and stirred for an additional $30 \mathrm{~min}$ at $90{ }^{\circ} \mathrm{C}$. Separately, $0.0035 \mathrm{~g}(0.0315 \mathrm{mmol})$ of $\mathrm{CH}_{3} \mathrm{NH}_{3} \mathrm{Br}$ and $0.037 \mathrm{~g}(0.1 \mathrm{mmol})$ of $\mathrm{PbBr}_{2}$ were dissolved in 100 and 200 $\mu \mathrm{L}$ DMF, respectively. The $\mathrm{CH}_{3} \mathrm{NH}_{3} \mathrm{Br}$ solution was added first and then allowed to react for $60 \mathrm{sec}$ before the addition of the $\mathrm{PbBr}_{2}$ solution. The solution color turned light yellow, at $60 \mathrm{sec}, 0.5 \mathrm{~mL}$ reaction mixture was injected into $5 \mathrm{~mL}$ acetone to quench the reaction. The yellow solid was centrifuged at $5000 \mathrm{rpm}$ for $5 \mathrm{~min}$, collected, and then again dissolved in toluene. The solution was centrifuged at $5000 \mathrm{rpm}$ for $2 \mathrm{~min}$, and the translucent yellow supernatant was collected. The solution was dried under a high vacuum and stored in a $20^{-} \mathrm{mL}$ vial capped under nitrogen.

\section{NWs Synthesis}

A $120 \mu \mathrm{L}$ of oleic acid $(0.38 \mathrm{mmol})$ was dissolved in $6 \mathrm{~mL}$ of 1 -ODE in a two-neck round bottom flask under $\mathrm{N}_{2}$. At $90{ }^{\circ} \mathrm{C}$, the reaction mixture was stirred for $45 \mathrm{~min}$. At this point, $0.0126 \mathrm{~g}(0.06 \mathrm{mmol})$ of solid $\mathrm{CH}_{3}\left(\mathrm{CH}_{2}\right)_{6} \mathrm{CH}_{2} \mathrm{NH}_{3} \mathrm{Br}$ was added to the reaction mixture. Then, stirred for an additional $30 \mathrm{~min}$ at $90{ }^{\circ} \mathrm{C}$. Separately, $0.0044 \mathrm{~g}(0.032 \mathrm{mmol})$ of $\mathrm{CH}_{3} \mathrm{NH}_{3} \mathrm{Br}$ 
and $0.037 \mathrm{~g}(0.1 \mathrm{mmol})$ of $\mathrm{PbBr}_{2}$ were dissolved in 100 and $200 \mu \mathrm{L} \mathrm{DMF}$, respectively. The $\mathrm{CH}_{3} \mathrm{NH}_{3} \mathrm{Br}$ solution was added first and then allowed to react for $60 \mathrm{sec}$ before the addition of the $\mathrm{PbBr}_{2}$ solution. The solution color turned light yellow, and after $240 \mathrm{sec} 0.5 \mathrm{~mL}$ reaction mixture was injected into $5 \mathrm{~mL}$ of acetone to quench the reaction. We followed the same purification procedure as described for $\mathrm{CH}_{3} \mathrm{NH}_{3} \mathrm{PbBr}_{3} \mathrm{NCs}$.

\section{NPS Synthesis}

$\mathrm{CH}_{3} \mathrm{NH}_{3} \mathrm{PbBr}_{3} \mathrm{NPs}$ were synthesized according to our published procedure. ${ }^{49}$ Briefly, $6 \mathrm{~mL}$ of ODE was stirred at $90{ }^{\circ} \mathrm{C}$ for $45 \mathrm{~min}$ and then $0.06 \mathrm{mmol}$ of solid $\mathrm{CH}_{3}\left(\mathrm{CH}_{2}\right)_{6} \mathrm{CH}_{2} \mathrm{NH}_{3} \mathrm{Br}$ was added to the reaction mixture and stirred for another $30 \mathrm{~min}$ at $90{ }^{\circ} \mathrm{C}$. Separately, $0.0044 \mathrm{~g}(0.032 \mathrm{mmol})$ of $\mathrm{CH}_{3} \mathrm{NH}_{3} \mathrm{Br}$ and $0.037 \mathrm{~g}(0.1 \mathrm{mmol})$ of $\mathrm{PbBr}_{2}$ were dissolved in 100 and $200 \mu \mathrm{L}$ DMF, respectively. The $\mathrm{CH}_{3} \mathrm{NH}_{3} \mathrm{Br}$ solution was added first and allowed to react for $60 \mathrm{sec}$ before the addition of the $\mathrm{PbBr}_{2}$ solution. The solution color turned light yellow, and after $60 \mathrm{sec}, 0.5 \mathrm{~mL}$ of the reaction mixture was injected into $5 \mathrm{~mL}$ acetone. We followed the same purification procedure as described for $\mathrm{CH}_{3} \mathrm{NH}_{3} \mathrm{PbBr}_{3} \mathrm{NCs}$.

TEM images of the perovskite samples are shown in Figure 3.1, while a histogram of size analysis of $\mathrm{CH}_{3} \mathrm{NH}_{3} \mathrm{PbBr}_{3} \mathrm{NCs}$ is given in Figure 3.2. The average dimensions of the nanostructures are as follows: NCs: $2.4 \pm 0.2 \mathrm{~nm}$ in 
diameter; NWs: $3.8 \pm 0.4 \mathrm{~nm}$ in diameter with a length range of 300 to $900 \mathrm{~nm}$;

NPs: $2.5 \pm 0.2$ in thickness with 20 to $50 \mathrm{~nm}$ in edge length.

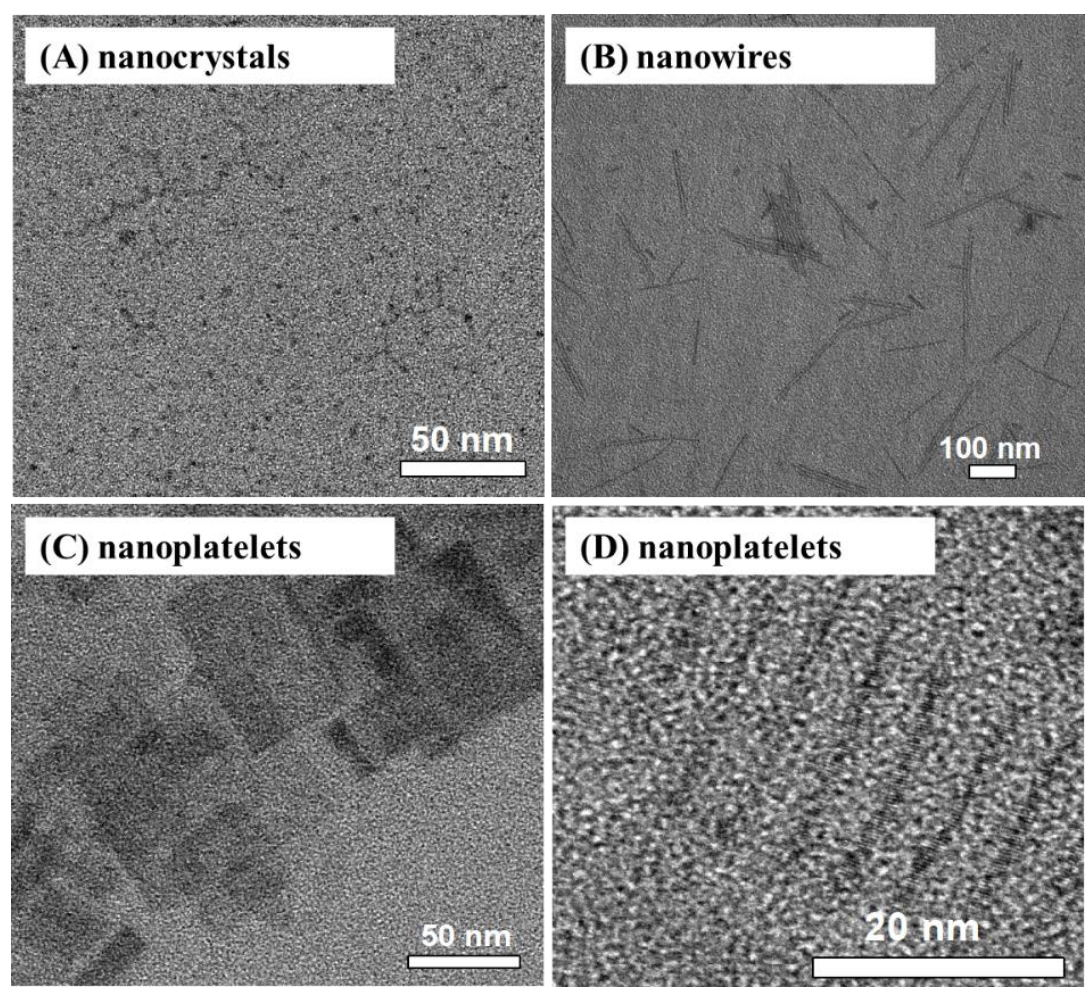

Figure 3.1. TEM images of perovskite nanostructures.

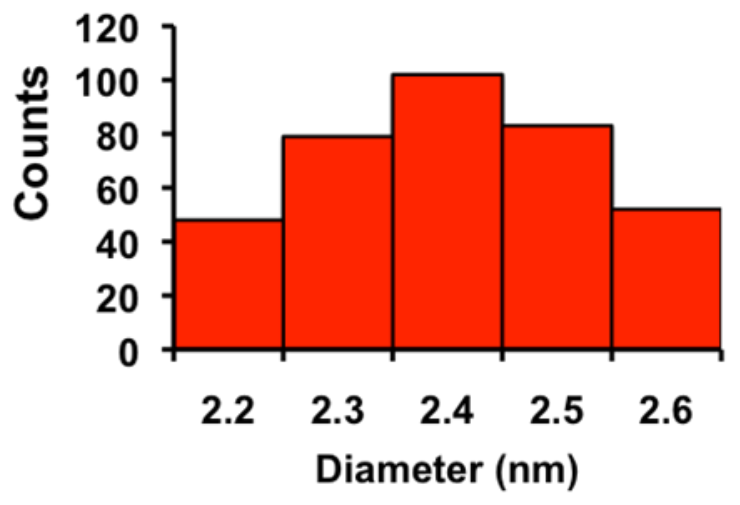

Figure 3.2. Histogram of size analysis of $\mathrm{CH}_{3} \mathrm{NH}_{3} \mathrm{PbBr}_{3} \mathrm{NCs}$ determined using the ImageJ software. Approximately $360 \mathrm{NCs}$ were counted for the analysis. The average size was determined to be $2.4 \pm 0.2 \mathrm{~nm}$. 
Steady-State Absorption and Photoluminescence Measurements.

Varian Cary 50 Scan UV-visible spectrophotometer was used to collect absorption spectra over the range of 300-800 $\mathrm{nm}$ using $1 \mathrm{~cm}$ quartz cuvettes. All spectra were collected in toluene, which was used as a background for these measurements. The PL emission spectra were acquired under illumination at $350 \mathrm{~nm}$ with a Cary Eclipse fluorescence spectrophotometer from Varian Instruments using $1 \mathrm{~cm}$ quartz cuvettes.

\section{Ultrafast transient absorption spectroscopy.}

Ultrafast TA pump-probe spectroscopy was employed to investigate the photoinduced processes in all three perovskite nanostructures. The samples were dissolved in toluene and contained in 1-mm-thick quartz cuvettes. The concentrations of the nanostructures were so adjusted that the transmission of the probe beam at the band-edge absorption peak was $10 \%$. The TA system ${ }^{107-}$ 108 (Clark-MXR, Shape Shifter) uses a regeneratively amplified mode-locked Ti:sapphire laser system (Clark-MXR, CPA, wavelength $=775 \mathrm{~nm}$, pulse duration $\lesssim 150 \mathrm{fs}$, pulse energy $1 \mathrm{~mJ}$ at $1 \mathrm{kHz}$ repetition rate). The fundamental output beam was split into three beams. One of them was frequency-doubled to $388 \mathrm{~nm}\left(\mathrm{~h} v_{\text {pump }}=3.2 \mathrm{eV}\right)$ and used as a pump source. The second beam was used to pump a non-collinear optical parametric amplifier (NOPA) and generate the second pump beam at $\sim 490 \mathrm{~nm}\left(\mathrm{~h} v_{\text {pump }}=2.5 \mathrm{eV}\right)$. Laser pulses from the NOPA were compressed to about $30 \mathrm{fs}$ pulse duration. 
Most of the measurements used the $388 \mathrm{~nm}$ pump beam in order to generate hot charge carriers, whereas the $490 \mathrm{~nm}$ beam was used for near-band-edge pumping. The other beam from the femtosecond amplifier was focused on a 3$\mathrm{mm}$-thick sapphire plate to generate a white-light continuum $(420-1600 \mathrm{~nm})$, which was used as the probe source. After the white-light generated, the 775 $\mathrm{nm}$ fundamental was filtered out by a laser mirror. The beams (pump and probe) were overlapped on the sample. Their polarizations are set to the magic angle. While the probe beam was focused on the sample, the pump beam was off-focused to a size of $\sim 0.5 \mathrm{~mm}$ in diameter on the sample for better coverage of the probe beam and to avoid sample damage and degradation. The probe beam was refocused into a spectrograph and detected in the wavelength range of 430-730 nm by a linear array CMOS detector (Imaging Solutions Group, LW-ELIS-1024A-1394). Before the sample, the pump beam was reflected by a retroreflector mounted on a computerized delay stage so that the delay time $(\Delta t)$ between the pump and the probe pulses can be scanned by moving the retroreflector. An optical chopper to modulate the pump beam at $500 \mathrm{~Hz}$ was used, half of the repetition rate of the femtosecond amplifier.

\subsection{Results and Discussion}

\subsubsection{Overview of the spectra}

\subsubsection{Steady-state Spectra}

UV-visible absorption and PL spectra of all three perovskite nanostructures are shown in Figure 3.3a. Peak centers, PL quantum yields, 
and radiative and non-radiative lifetimes are listed in Table 3.1. The morphology of the nanostructures affects their absorbance and PL quantum yields. PL quantum yield of NCs (16\%) is significantly lower than NWs (59\%) and NPs (44\%), which is attributed to the delocalization of the exciton wavefunction. Note that any unpassivated surface sites on the NCs would result in the formation of midgap trap states that also reduces PL quantum yield. However, no evidence of significant contribution from trap states was observed in our studies (see Section Role of the trap states of this chapter). This is because the small radius of curvature of NCs allows sufficient packing of surface passivating ligands by avoiding the steric hindrance, and hence prevents trap state formation. 


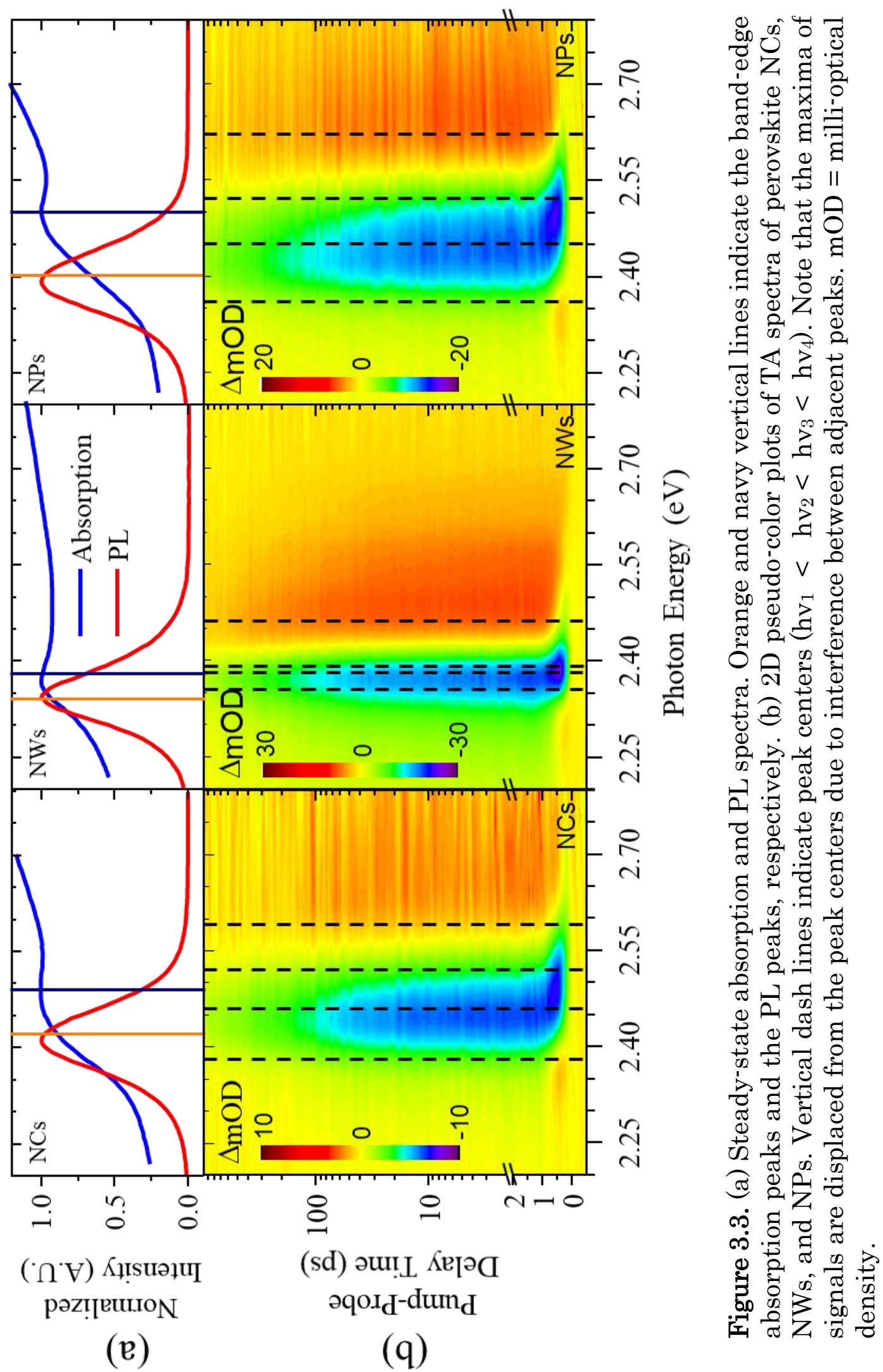


Limited dimensions of the nanostructures and the resulting quantum confinement lead to blue-shift of the absorption onsets and PL peaks (Figure 3.3a) compared to bulk $\mathrm{CH}_{3} \mathrm{NH}_{3} \mathrm{PbBr}_{3}$ perovskite single crystals $(\mathrm{Eg}=2.2$ eV).109-110 The blue-shifts are smaller for NWs because of their large lengths and hence, weaker quantum confinement along that dimension. The quantum confinement effect also results in a semi-discrete energy level structure, as evidenced by the prominent band-edge exciton peaks in the UV-visible absorption spectra that are assigned to $1 \mathrm{~s}(\mathrm{e})-1 \mathrm{~s}(\mathrm{~h})$ transitions..$^{111-112}$

All three UV-visible absorption spectra have sub-band-gap tails extended into the red region. By contrast, step onsets were observed in the absorption spectra of bulk $\mathrm{CH}_{3} \mathrm{NH}_{3} \mathrm{PbBr}_{3}$ perovskite single crystals. ${ }^{105,109-112}$ The tails observed in the present measurements follow the Urbach rule ${ }^{113}$ and decay exponentially. Urbach tails were observed in the absorption spectra of perovskite thin films ${ }^{114}$ as well as nanostructures of other semiconductors. ${ }^{115}$ Such exponential decay in the absorption edge of semiconductor materials can be explained using conduction electron-optical phonon interaction. ${ }^{116}$ However, Quantitative understanding and modeling of the Urbach-tails requires a temperature-dependence measurements ${ }^{114}$ and was not attempted in the present work. Nevertheless, it is worth noting that the UV-visible absorption spectrum of NWs possesses a much stronger tail than the other two nanostructures, indicating stronger electron-phonon interaction. Furthermore, in all three UV/visible absorption spectra, a background absorption is 
discernable, which is due to scattering from large superstructures (pearnecklace assemblies of NCs, bundles of NWs, and stacked NPs) that are present in the solutions. ${ }^{117-118}$

Table 3.1. Peak centers in UV-visible absorption (Abs.) and photoluminescence (PL) spectra, photoluminescence quantum yields $\left(\Phi_{\mathrm{FL}}\right)$, and radiative lifetimes $(\tau)$ of $\mathrm{CH}_{3} \mathrm{NH}_{3} \mathrm{PbBr}_{3}$ perovskite nanostructures.

\begin{tabular}{cccccccc} 
Nanostructure & & & & & & $\tau_{\text {fast }}$ & $\tau_{\text {slow }}$ \\
& $(\mathrm{nm})$ & $(\mathrm{eV})$ & $(\mathrm{nm})$ & $(\mathrm{eV})$ & $(\%)$ & $(\mathrm{ns})$ & \\
\hline $\mathrm{NCs}$ & 499 & 2.49 & 513 & 2.42 & $16 \pm 2$ & $9 \pm 2$ & $50 \pm 5$ \\
\hline $\mathrm{NWs}$ & 522 & 2.38 & 529 & 2.34 & $59 \pm 7$ & $11 \pm 1$ & $89 \pm 13$ \\
\hline $\mathrm{NPs}$ & 497 & 2.5 & 515 & 2.41 & $44 \pm 3$ & $10 \pm 1$ & $63 \pm 8$
\end{tabular}

Radiative $\left(\tau_{\mathrm{r}}\right)$ and non-radiative $\left(\tau_{\mathrm{nr}}\right)$ lifetimes were calculated from the measured PL lifetimes $\left(\tau_{\mathrm{PL}}\right)$ and quantum yields $\left(\Phi_{\mathrm{FL}}\right)$

For all three nanostructures, PL peaks demonstrate a Stokes shift with respect to the absorption peaks. It is attributed mainly to the removal of degeneracy in the band-edge exciton fine structure due to nanostructure asymmetry and the electron-hole exchange interaction. ${ }^{119-122}$ The energy difference between the band-edge absorption peak and the PL peak corresponds to the separation between the upper optically active (or "bright") levels with large oscillator strength and the lower optically passive (or "dark") levels in the exciton ground state. The magnitude of the redshift is strongly 
affected by the size and shape of the nanostructures. Comparing the three nanostructures, it is uniquely small for NWs because of their large length.

\subsubsection{Transient absorption Spectra}

TA spectra of $\mathrm{CH}_{3} \mathrm{NH}_{3} \mathrm{PbBr}_{3}$ perovskite nanostructures obtained with a $388 \mathrm{~nm}$ pump $\left(\mathrm{hv}_{\text {pump }}=3.2 \mathrm{eV}\right)$ and white-light probe beams are illustrated in Figure $3.3 \mathrm{~b}$ in comparison with their steady-state spectra. TA spectra in Figure $3.3 \mathrm{~b}$ were obtained with $20 \mathrm{\mu J} / \mathrm{cm}^{2}$ pump fluence, and the transmission of the pump beam was $\sim 50 \%$. TA spectra of all three nanostructures crystals, wires, and platelets share some common features. The high probe photon energy region is dominated by a broad positive signal (centered at $\mathrm{hv}_{4}$ in Figure 3.3b) corresponding to photoinduced intraband absorption. In the low probe photon energy region, a strong negative signal is observed, which may be attributed to state filling (SF) or stimulated emission (SE) or both. A more careful inspection reveals that the negative signal comprises a fast (sub-picosecond) component to the blue side (at $\mathrm{hv}_{3}$ ) and a slow component to the red side (at $\mathrm{hv}_{2}$ ) that lasts for the whole time window of detection ( $\left.1 \mathrm{~ns}\right)$. In addition, a weak and fast (sub-picosecond) positive photoinduced absorption (PIA) signal $\left(a t h v_{1}\right)$ is discernible to the red of the strong negative signal.

Although the four components are present in all three TA spectra, the peak centers and linewidths differ between nanostructures. First, the linewidth of the negative signal in the TA spectrum of NWs is significantly 
narrower than the other two. This coincides with the smaller Stokes shift of the PL peak of NWs with respect to their band-edge absorption peak. The other major difference between the three nanostructures is that the peak center of the slower negative signal $\left(a t \mathrm{hv}_{2}\right)$ in the TA spectra of NCs and NPs approximates the average of the centers of the band-edge absorption and PL peaks, whereas in the TA spectrum of NWs it is better aligned with the bandedge absorption peak. Because the centers of SF and SE are expected to match those of the band-edge absorption and PL peaks, respectively, the TA spectroscopic observations described above suggest that both SF and SE contribute significantly to the negative signal in the TA spectra of NCs and NPs, whereas that in the TA spectrum of NWs is dominated by SF. Although both SF and SE can induce negative signals in TA spectra, SE cannot occur unless the carrier density threshold is reached.123-124 Due to their larger dimensions and weaker quantum confinement, the SE threshold is significantly higher for NWs than NCs and NPs, which explains the blueshifted center and narrower linewidth of the slower negative signal in the TA spectrum of NWs.

\subsection{Transient Kinetics}

\subsubsection{Thermalization and cooling of hot charge carriers}

Transient kinetics of the three nanostructures at four-probe photon energies are shown in Figure 3.4. Rise times of the PIA signal near hv 1 and the $\mathrm{SF}$ signal near $\mathrm{hv}_{3}$ are limited by instrument response function (IRF) of the 
spectrometer (FWHM $\sim 350 \mathrm{fs}$ ). The decay of these two components of the TA spectra coincides with the continued growth of the SF/SE signal near hv2 and the intraband absorption signal near $\mathrm{hv}_{4}$, which reaches their maxima at $\sim 1$ ps and are followed by slow decay.

Early-stage photoinduced dynamics in semiconductor nanostructures have been well studied, ${ }^{125-126}$ and are illustrated in Figure 3.5 and summarized in figure 3.6. Photoexcitation above the bandgap creates hot electrons in the conduction band and hot holes in the valence band. After photoexcitation, electrons and holes thermalize to a quasi-equilibrium distribution, namely, a Fermi-Dirac distribution with a high charge carrier temperature, on a timescale $\left(\tau_{\text {th }}\right)$ much shorter than one ps. ${ }^{127-128}$ Charge carrier temperature could be determined by fitting the blue tail of the SF signal to the Boltzmann distribution. ${ }^{129}$ However, it was not attempted in the present work due to spectral congestion. Hot charge carriers then undergo re-distribution and cooling. The time constant for the cooling process $\left(\tau_{c}\right)$ has been found to be $\sim 230$ fs in the $\mathrm{CH}_{3} \mathrm{NH}_{3} \mathrm{PbI}_{3}$ perovskite thin film, ${ }^{129}$ which is close to the decay time at $\mathrm{hv}_{1}$ and $\mathrm{hv}_{3}$ and the rise time at $\mathrm{hv}_{2}$ and $\mathrm{hv}_{4}$.

Based on the kinetics model outlined above, the sub-picosecond negative signal at hv3 is attributed to $\mathrm{SF}$ due to hot electrons and holes at quasiequilibrium following charge carrier thermalization. Since the maxima of the high-temperature distribution are displaced from the band edges, the center of the $\mathrm{SF}$ signal so induced $\left(\mathrm{hv}_{3}\right)$ is blue-shifted from the band-edge absorption 
peak. The subsequent sub-picosecond decay at hv 3 as well as growth at $\mathrm{hv}_{2}$ and $\mathrm{hv}_{4}$ is attributed to the cooling of the hot charge carriers. As electrons and holes redistribute toward band edges, the center of the SF/SE signal moves toward lower energy (from hv $\mathrm{h}_{3}$ to $\mathrm{hv}_{2}$ ).

PIA signal at $\mathrm{hv}_{1}$ can be attributed to photoinduced bandgap renormalization (BGR). Interaction between hot electrons and holes induces new band edges and reduces the bandgap. Immediately following the photoexcitation, the states near the new band edges are not fully occupied. Transitions into these states lead to PIA at $\mathrm{hv}_{1}$, which decays at the same rate as the SF signal at hv ${ }_{3}$ due to charge carrier cooling. 


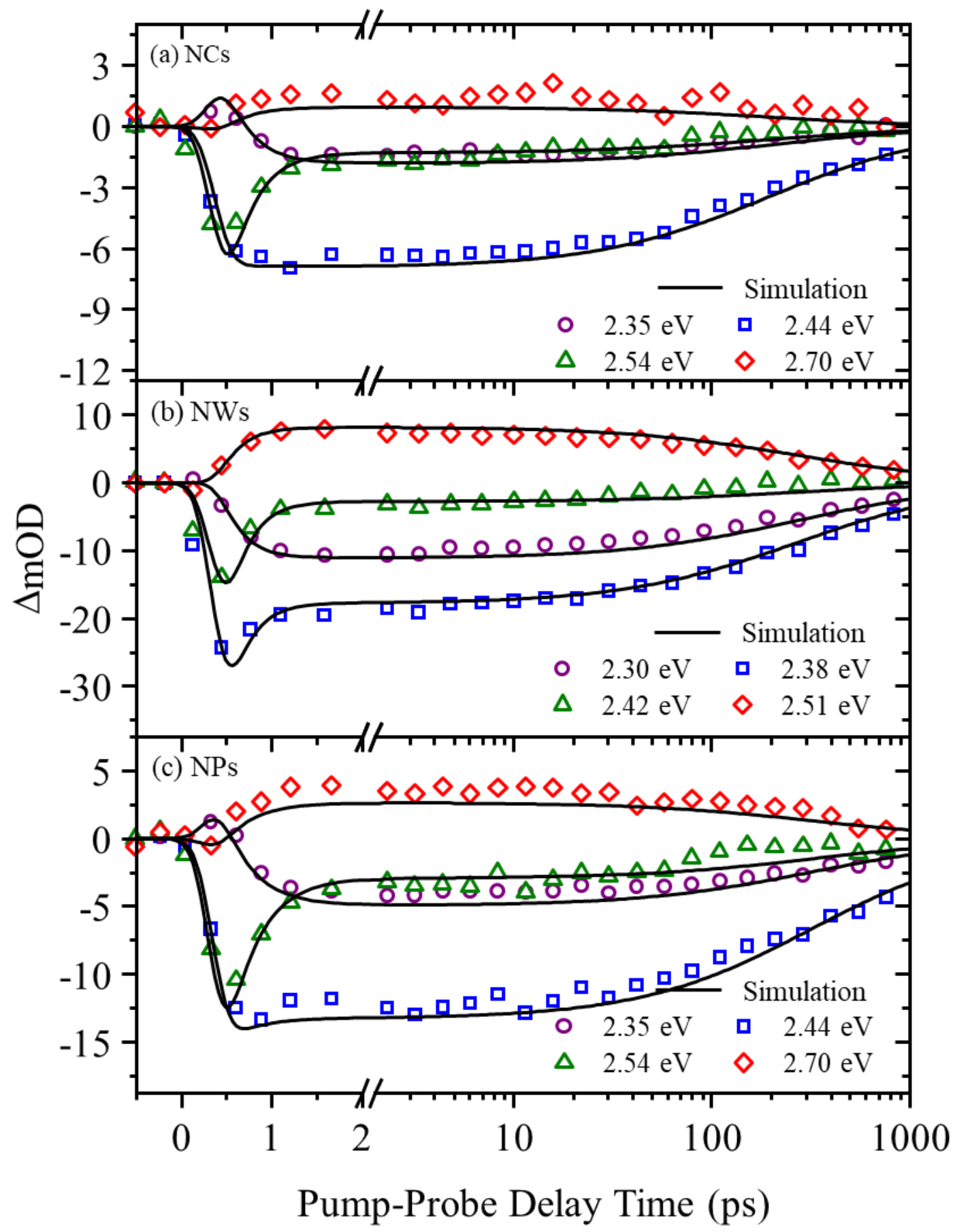

Figure 3.4. Transient kinetics of (a) NCs, (b) NWs, and (c) NPs at four probe photon energies. Thin lines are simulations using parameters determined in global fitting. 
Other photoinduced processes such as electro-absorption from the electric field of photo-generated correlated charge pairs may also cause subband-gap PIA. ${ }^{130-131}$ The current attribution of PIA at $\mathrm{hv}_{1}$ to BGR is supported by an important control experiment using a pump beam with lower photon energy $\left(h v_{\text {pump }}=2.5 \mathrm{eV}\right)$. TA spectra and transient kinetics at $h v_{\text {pump }}=2.5 \mathrm{eV}$ in comparison with $\mathrm{hv}_{\text {pump }}=3.2 \mathrm{eV}$ is shown in figure 3.5. The positive PIA feature at $\mathrm{hv}_{1}$ disappears with this near-band-edge pump photon energy, a phenomenon that was also observed in the TA spectra of $\mathrm{CH}_{3} \mathrm{NH}_{3} \mathrm{PbI}_{3}$ perovskite thin films ${ }^{128}$ and used to support the hypothesis of BGR. Bi-exciton interaction may also induce sub-band-gap PIA. ${ }^{132-134}$ However, it is a less plausible explanation than BGR because of the relatively low exciton density in the three nanostructures. Figure 3.6 summarizes the photoinduced processes that occur in the perovskite nanostructures upon excitation. 

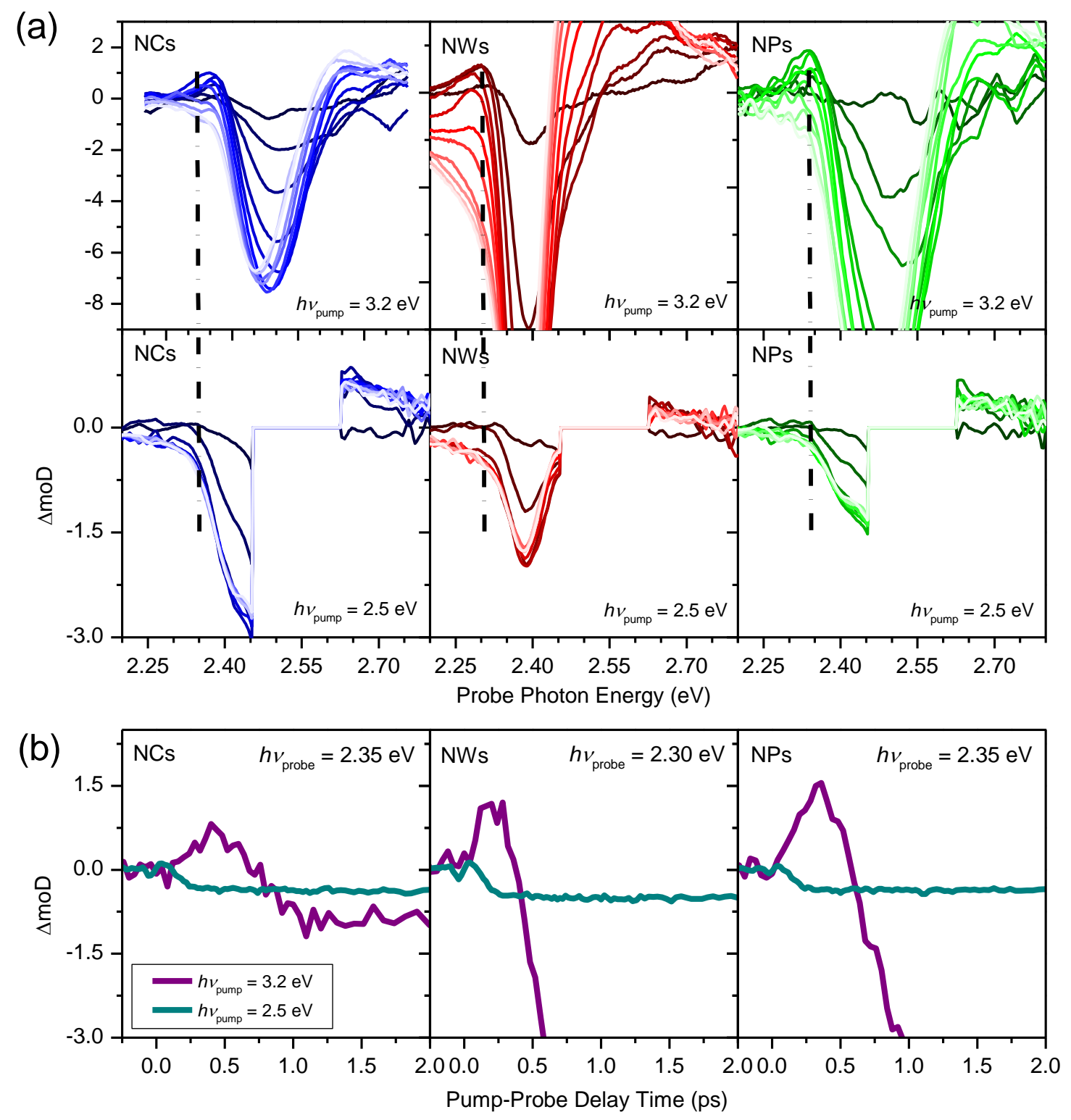

Figure 3.5. (a) Comparison between TA spectra with two pump photon energies: $3.2 \mathrm{eV}$ (top) and $2.5 \mathrm{eV}$ (bottom). The pump-probe delay times $(\Delta \mathrm{t})$ are between 0 and $1 \mathrm{ps}$. Vertical dash lines indicate the center of the photo-induced absorption (PIA) signal $\left(h v_{1}\right)$. (b) Comparison between transient kinetics at $h v_{1}$ with the two pump photon energies. Note that the PIA signal is absent with $\mathrm{h} v_{\text {pump }}=2.5 \mathrm{eV}$. 


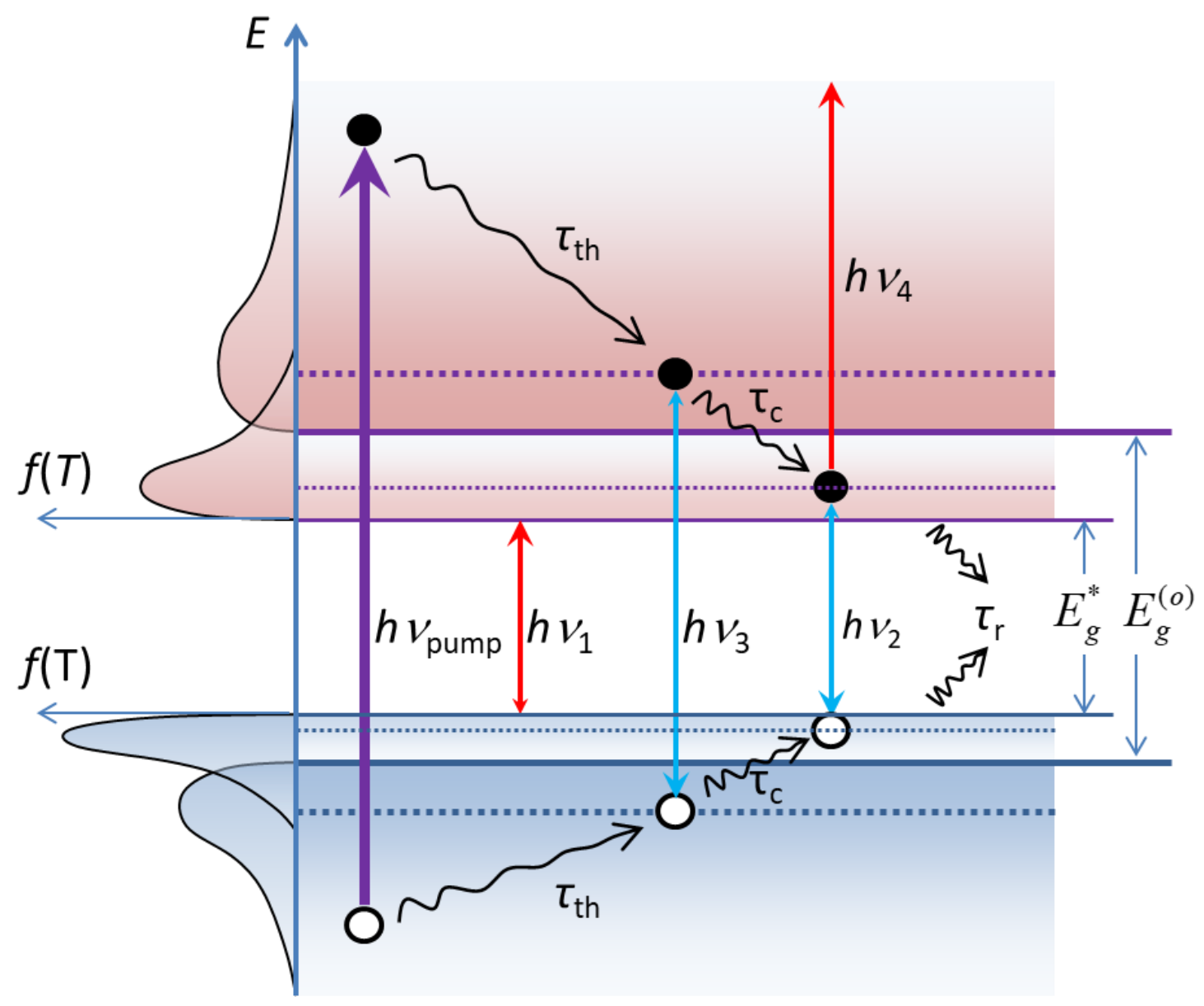

Figure 3.6. Energy diagram and photoinduced processes in perovskite nanostructures. Straight arrows mark the photon energies of the pump beam and the peaks in the TA spectra. Wavy arrows indicate charge carrier relaxation processes. Thick and thin solid horizontal lines are the original and renormalized band gaps, respectively. $\mathrm{f}(\mathrm{T})$ : charge carrier distribution.

\subsubsection{Charge recombination process}

The thermalization and cooling of hot charge carriers are followed by the recombination process that is responsible for the decay of both the SF/SE signal at $\mathrm{hv}_{2}$ and the intraband absorption signal at $h v_{4}$. Both decay processes can be well reproduced by a hyperbolic function, suggesting second-order kinetics. By 
contrast, fitting the decay processes to either first- or third-order kinetics introduces systematic errors (see Figure 3.7). The density of the charge carriers in the recombination process can, therefore, be written as:

$$
[n](\Delta t)=\frac{\left[n_{0}\right]}{1+\Delta t / \tau_{1 / 2, r}}=\frac{\left[n_{0}\right]}{1+\left[n_{0}\right] k_{r} \Delta t}
$$

Where $[\mathrm{n}](\Delta \mathrm{t})$ is the charge carrier density at delay time $\Delta \mathrm{t},\left[\mathrm{n}_{0}\right]$ is the initial density, and $\mathrm{k}_{\mathrm{r}}$ is the effective rate constant of the bimolecular reaction. The half-time is $\tau_{1 / 2, \mathrm{r}}=1 /\left(\left[\mathrm{n}_{0}\right] \mathrm{k}_{\mathrm{r}}\right)$.

Under our experimental conditions, namely a pump fluence of $20 \mu \mathrm{J} / \mathrm{cm}^{2}$ and $\sim 50 \%$ absorption for the pump beam, the charge carrier density is estimated to be $\sim 2 \times 10^{18} \mathrm{~cm}^{-3}$. 
(a)

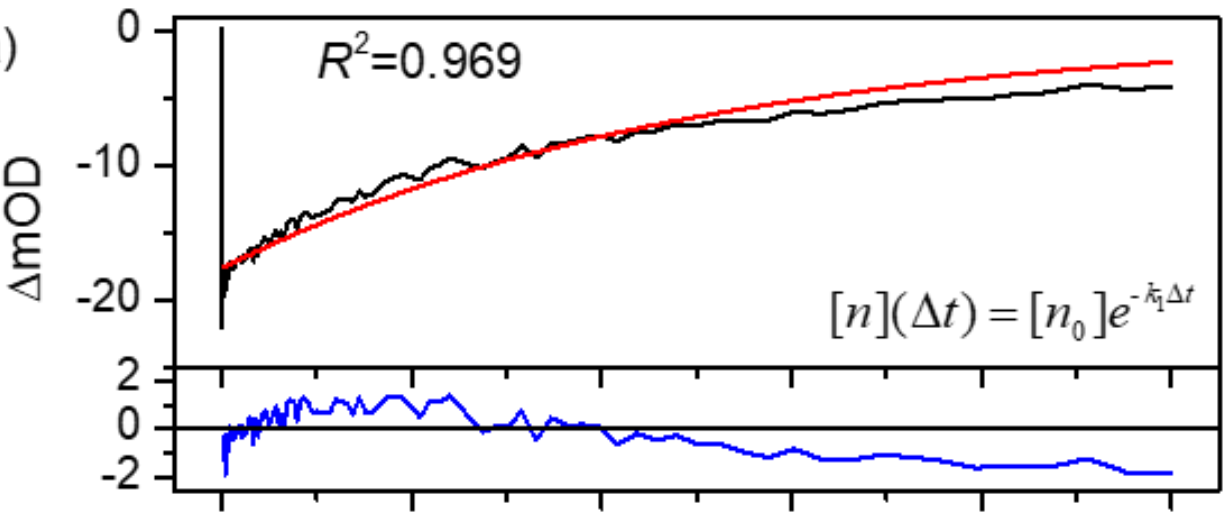

(b)

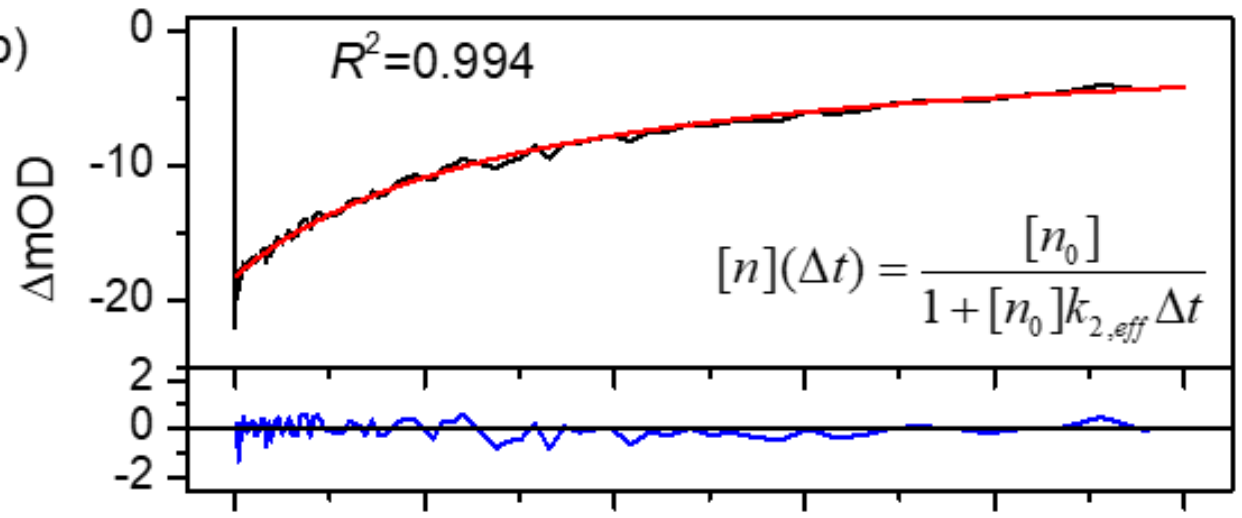

(c)

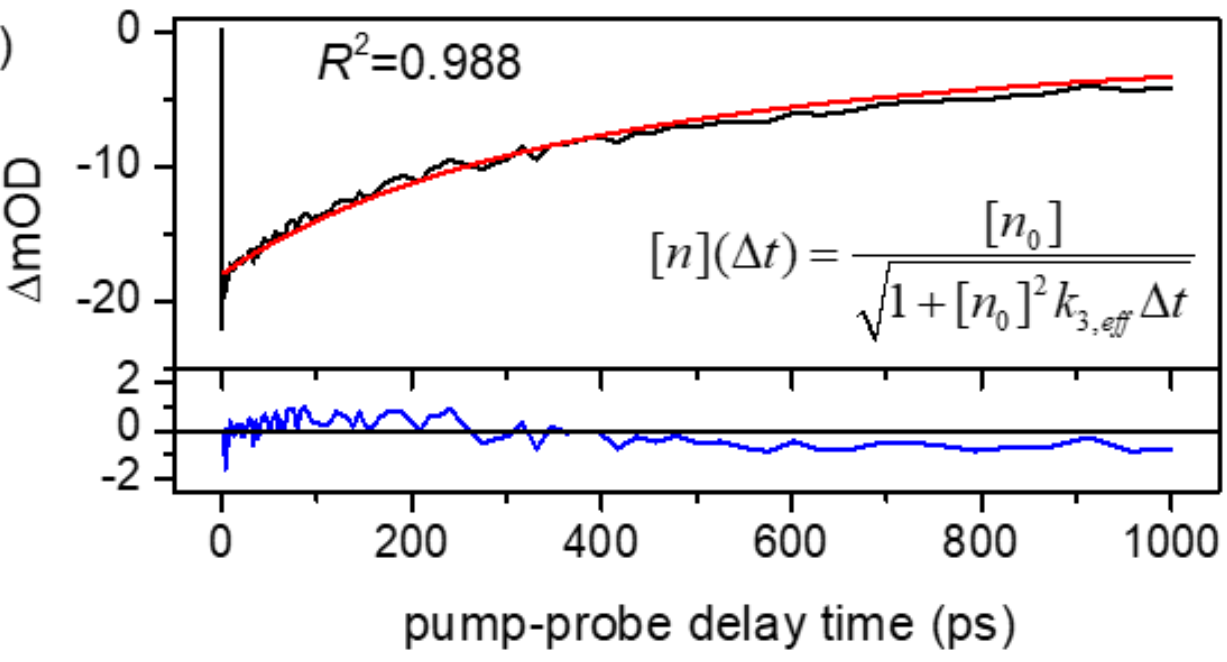

Figure 3.7. Decay curve at hv2 of the TA spectrum of NWs at $20 \mu \mathrm{J} / \mathrm{cm}^{2}$ pump fluence fit to the concentration profile of the reactant of (a) a first order reaction (for geminate recombination); (b) a second-order reaction (for non-geminate recombination), and (c) a third-order reaction (for Auger recombination) along with residuals. The secondorder reaction model gives the best fit while systematic errors are introduced using the other two. Similar observations have been made for other nanostructures and/or at another pump fluences. 
By fitting the decay curves of the SF/SE signal at $\mathrm{hv}_{2}$, the half-times of the charge carrier recombination process $\left(\tau_{1 / 2, \mathrm{r}}\right)$ is determined to be 191, 293, and 332 ps for NCs, NWs, and NPs, respectively. Both the decay function and timescale of the observed TA kinetics suggest that charge recombination in the investigated perovskite nanostructures is a bimolecular process that obeys the rate law of a second-order reaction. The experimentally determined recombination rate is significantly faster than that in perovskite thin films, but still, three orders of magnitude slower than the Langevin rates determined by assuming free electron-hole annihilation. ${ }^{135-136}$

Charge recombination processes in perovskite thin films have been extensively investigated and reviewed (see Ref. 138 and references therein). First-order (monomolecular geminate) recombination, second-order (bimolecular non-geminate) recombination, and third-order (Auger recombination) may all occur in thin films depending on the charge carrier density.40,137 Given the relatively low charge carrier densities in our experiment, the absence of multiexciton Auger recombination is well justified. However, the absence of geminate recombination, a monomolecular process, is puzzling at first glance. The timescale of geminate recombination is $\sim 0.1 \mu \mathrm{s}^{135}$ and two orders of magnitude longer than the time window of our ultrafast measurements ( $1 \mathrm{~ns})$. Therefore, geminate recombination, if present, would manifest itself as a constant offset in our TA spectra, which was not observed. Previously, the trap-assisted monomolecular recombination process at a few 
nanoseconds was observed in the TA spectra of $\mathrm{CH}_{3} \mathrm{NH}_{3} \mathrm{PbI}_{3}$ thin films. ${ }^{137}$ Such a slow decay component is not present in our TA kinetics either.

The absence of a geminate recombination process in the TA kinetics, however, is not unexpected. By correlating femtosecond TA and X-ray absorption measurements, Zheng et al. ${ }^{97}$ have revealed the photoinduced formation of strong-coupling polarons in $\mathrm{CH}_{3} \mathrm{NH}_{3} \mathrm{PbBr}_{3}$ nanoparticles due to carrier-lattice interaction. Such strong coupling polarons comprise the net lattice distortion and the self-trapped charge carriers. Hybrid density functional theory (DFT) calculations predict that electrons are more readily trapped as polarons than holes in charged isolated $\mathrm{CH}_{3} \mathrm{NH}_{3} \mathrm{PbBr}_{3}$ clusters. ${ }^{138}$ Electrons and holes are therefore separated spatially in the nanostructures. Based on their experimental and computational observations, Zheng et al. ${ }^{97}$ predicted that in $\mathrm{CH}_{3} \mathrm{NH}_{3} \mathrm{PbBr}_{3}$ nanostructures, (i) geminate recombination is less probable, while (ii) rates of the non-geminate and Auger recombination processes depend on charge carrier concentrations.

The fact that the charge recombination process observed at hv2 and $h v_{4}$ in the present TA measurement can be modeled using just a second-order bimolecular process verifies their first prediction. Their second prediction has been verified by pump-fluence dependence measurements, discussed in the next section of this chapter. 


\subsection{Pump fluence dependence study}

Pump fluence dependence measurements can provide critical information about the nature of photoinduced processes. ${ }^{40,131}$ Transient kinetics near hvs under different pump fluences $\left(\mathrm{F}_{\text {pump }}\right)$ are compared in Figure 3.8. Rise times of the $\mathrm{SF}$ signal $(\sim 500 \mathrm{fs}$, defined as from time zero to its maximum), is comparable to the IRF so that change in the charge carrier thermalization time constant $\left(\tau_{\text {th }}\right)$ due to different $F_{\text {pump }}$, i.e., different initial charge carrier densities $\left[\mathrm{n}_{0}\right]$, was not detected.

The difference in the charge carrier cooling rate $\left(\mathrm{k}_{\mathrm{c}}\right)$ detected under different pump fluences is moderate but ineligible. Using the model outlined in Section 3.4 and illustrated in Figure 3.6 Kinetic model, the sub-picosecond decay at $\mathrm{hv}_{3}$, as well as the growth at $\mathrm{hv}_{2}$ and $\mathrm{hv}_{4}$, is due to the cooling process. The decay time constant at $\mathrm{hv}_{3}$ increases from $\sim 200$ fs at low pump fluence to $\sim 400$ fs at high pump fluence (with IRF included). Decrease of the cooling rate upon high pump fluence, i.e., high initial charge carrier density, has been observed in perovskites thin films and was attributed to a hot phonon bottleneck effect,127-128 which was also observed in inorganic crystalline semiconductors. 139 


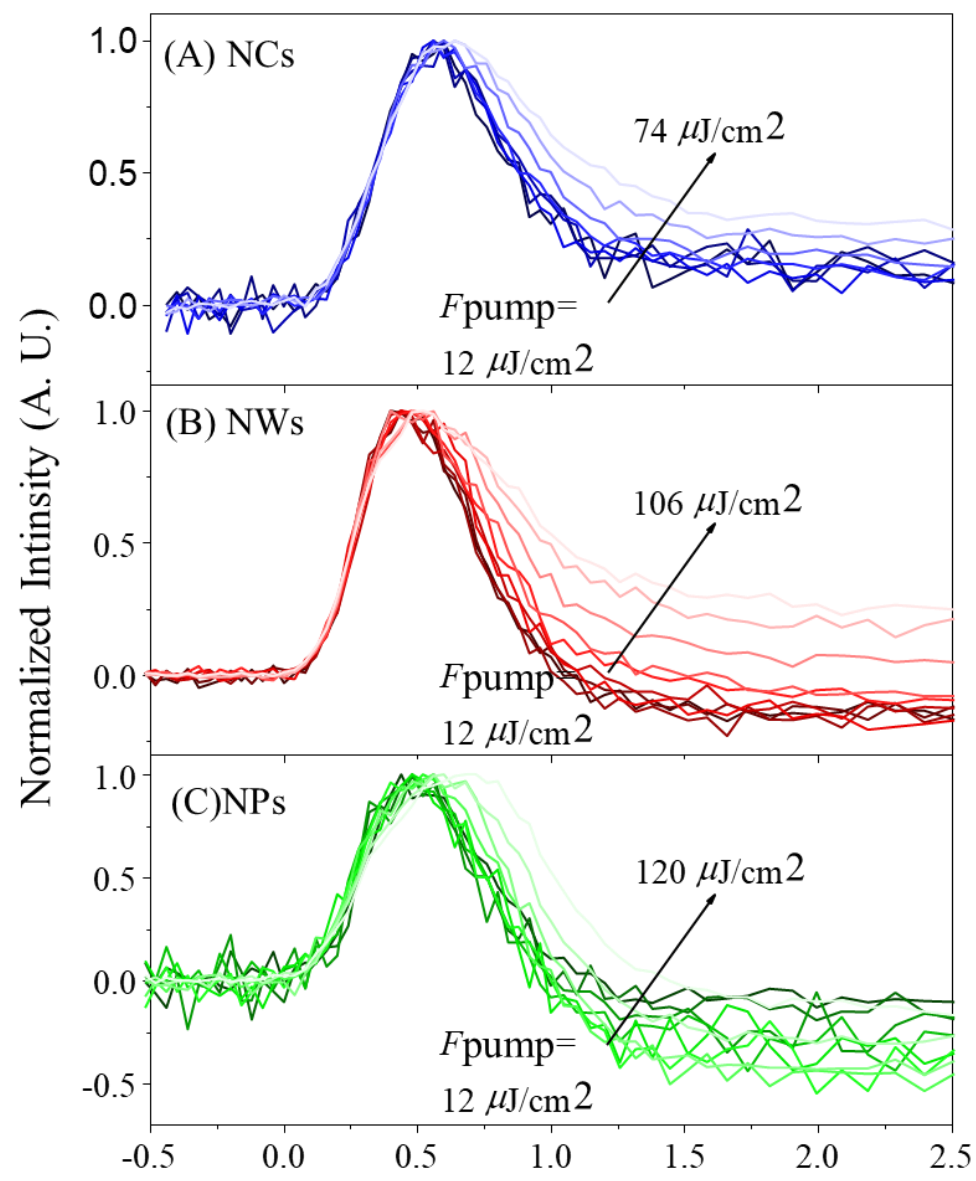

Pump-Probe Delay Time (ps)

Figure 3.8. Transient kinetics of (a) NCs, (b) NWs, and (c) NPs at hv 3 under different pump fluences. The TA signals are normalized to its maximum at $\sim 0.5 \mathrm{ps}$. 
At high $\left[n_{0}\right]$, optical phonons may be reabsorbed by charge carriers, leading to the reduced cooling rate. Decay time at hvs owing to charge carrier cooling $\left(\tau_{c}\right)$ increases moderately under higher pump fluences, i.e., with higher initial charge carrier densities $\left[\mathrm{n}_{0}\right]$ (see Figure 3.8). Decay processes between $750 \mathrm{fs}$ and $5 \mathrm{ps}$ for all three samples under different pump fluences are fit to an exponential decay function. The rate constants $\mathrm{k}_{\mathrm{c}}$ determined in the fittings are plotted as a function of $\left[\mathrm{n}_{0}\right]$ in Figure 3.9. As described above, $\left[\mathrm{n}_{0}\right]$ is estimated to be $\sim 2 \times 10^{18} \mathrm{~cm}^{-3}$ for a pump fluence of $20 \mu \mathrm{J} / \mathrm{cm}^{2}$ and assumed to be linearly proportional to the maximum of the strong SF/SE signal at $\mathrm{hv}_{2}$. Similar values for $\left[\mathrm{n}_{0}\right]$ were obtained using maxima at other peak wavelengths.

A linear relation is observable between $\mathrm{k}_{\mathrm{c}}$ and $\left[\mathrm{n}_{0}\right]$ for all three samples, which can be written as:

$$
k_{c}=k_{c}^{(0)}+\chi_{c}\left[n_{0}\right]
$$

Where $k_{c}^{(0)}$ is the rate constant at the limit of zero charge carrier, and $\mathrm{X}_{\mathrm{c}}$ is the linearity coefficient. And $X_{c}$ of all three perovskite nanostructures studied for the three samples are determined in weighted linear fittings and listed in Table 3.2. Comparing three nanostructures, $\mathrm{kc}$ of $\mathrm{NCs}$ is the most sensitive to $\left[\mathrm{n}_{0}\right]$ due to stronger quantum confinement. 
Rate constants reported in Figure 3.9 and Table 3.2 demonstrate the influence of the pump fluence and hence, the initial charge carrier density on the cooling process, which is attributed to the hot phonon bottleneck effect.

Table 3.2. Charge carrier cooling rate constants at the zerodensity limit $\left(k_{c}^{(0)}\right)$ and linearity coefficients $X_{c}$ of $\mathrm{CH}_{3} \mathrm{NH}_{3} \mathrm{PbBr}_{3}$ perovskite nanostructures determined in fitting the transient kinetics.

\begin{tabular}{ccc} 
Nanostructure & $k_{c}^{(0)}$ & $\mathrm{Xc}$ \\
& $\left(\mathrm{ps}^{-1}\right)$ & $\left(\mathrm{cm}^{3} \mathrm{ps}^{-1}\right)$ \\
\hline $\mathrm{NCs}$ & $4.94(13)$ & $-0.47(3)$ \\
\hline $\mathrm{NWs}$ & $4.45(11)$ & $-0.26(2)$ \\
\hline $\mathrm{NPs}$ & $5.02(16)$ & $-0.25(2)$ \\
\hline \hline
\end{tabular}

However, the rate constants determined in the fittings mentioned earlier, especially those at low pump fluences, are not an accurate measure of the charge carrier cooling process for three reasons:

(1) The cooling process is coupled to the thermalization process. Overall TA signal at hv 3 obeys the rule for the reaction intermediate of a consecutive two-step reaction;

(2) The observed TA signal is convolved with IRF, a timescale of which (FWHM $350 \mathrm{fs}$ ) is comparable to the decay constants at hv3 $(<450 \mathrm{fs})$; 
(3) The long-delay-time baseline at $\mathrm{hv}_{3}$ is affected by the strong signals at $h_{2}$ and $h_{4}$ and varies when the pump fluence is changed. An accurate determination of kc requires TA measurements with significantly smaller IRF. In addition, fitting of the experimentally obtained spectra to a comprehensive model that combines all involved processes - instead of a single exponential decay - is necessary.

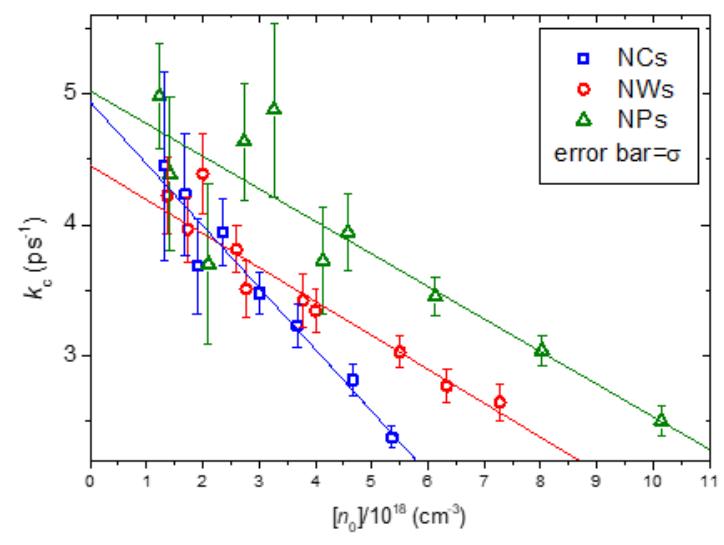

Figure 3.9. Charge carrier cooling rate constants $\left(\mathrm{k}_{\mathrm{c}}\right)$ for $\mathrm{NCs}$ (blue squares), NWs (red circles) and NPs (green triangles) at different initial charge carrier density $\left[\mathrm{n}_{0}\right]$. Error bars represent one standard deviation (rms) of fitting the decay signal at hv 3 to an exponential function. Solid lines are fittings to a linear function. Data points are weighted by $1 / \sigma^{2}$ in the fittings.

Compared to thermalization and cooling processes, the half-time for the charge carrier recombination $\left(\tau_{1 / 2, \mathrm{r}}\right)$ shows a much stronger dependence on the pump fluence. By fitting the decay process at $\mathrm{hv}_{2}$ to Equation 2, shown in figure 3.10, $\tau_{1 / 2, \mathrm{r}}$ under different pump fluences can be determined (Figure 3.11a). It decreases from $\sim 500 \mathrm{ps}$ at high pump fluence to $\sim 50 \mathrm{ps}$ at low pump fluence. 
Also determined in the fitting are the maxima of the SF/SE signal at hv2 (Figure $3.11 b)$. Since $\tau_{1 / 2, r}=1 /\left(\left[n_{0}\right] k_{r}\right)$, the half-time is affected by not only the effective rate constant $\left(\mathrm{k}_{\mathrm{r}}\right)$ but also the initial carrier density following photoexcitation $\left(\left[\mathrm{n}_{0}\right]\right)$. The estimated value of $\left[\mathrm{n}_{0}\right]$ of our samples is $\left[\mathrm{n}_{0}\right] \sim 2 \times 10^{18}$ $\mathrm{cm}^{-3}$ for a pump fluence of $20 \mathrm{\mu J} / \mathrm{cm}^{2}$ and assumed to be linearly proportional to the maximum of the SF/SE signal at $\mathrm{hv} 2$.

Numerical values of $\mathrm{k}_{\mathrm{r}}$ at different $\left[\mathrm{n}_{0}\right]$ can, therefore, be determined and are illustrated in Figure 3.12. Quality of the fittings again suggests that the contribution of multiphoton absorption and Auger recombination to the observed ultrafast dynamics is negligible. 


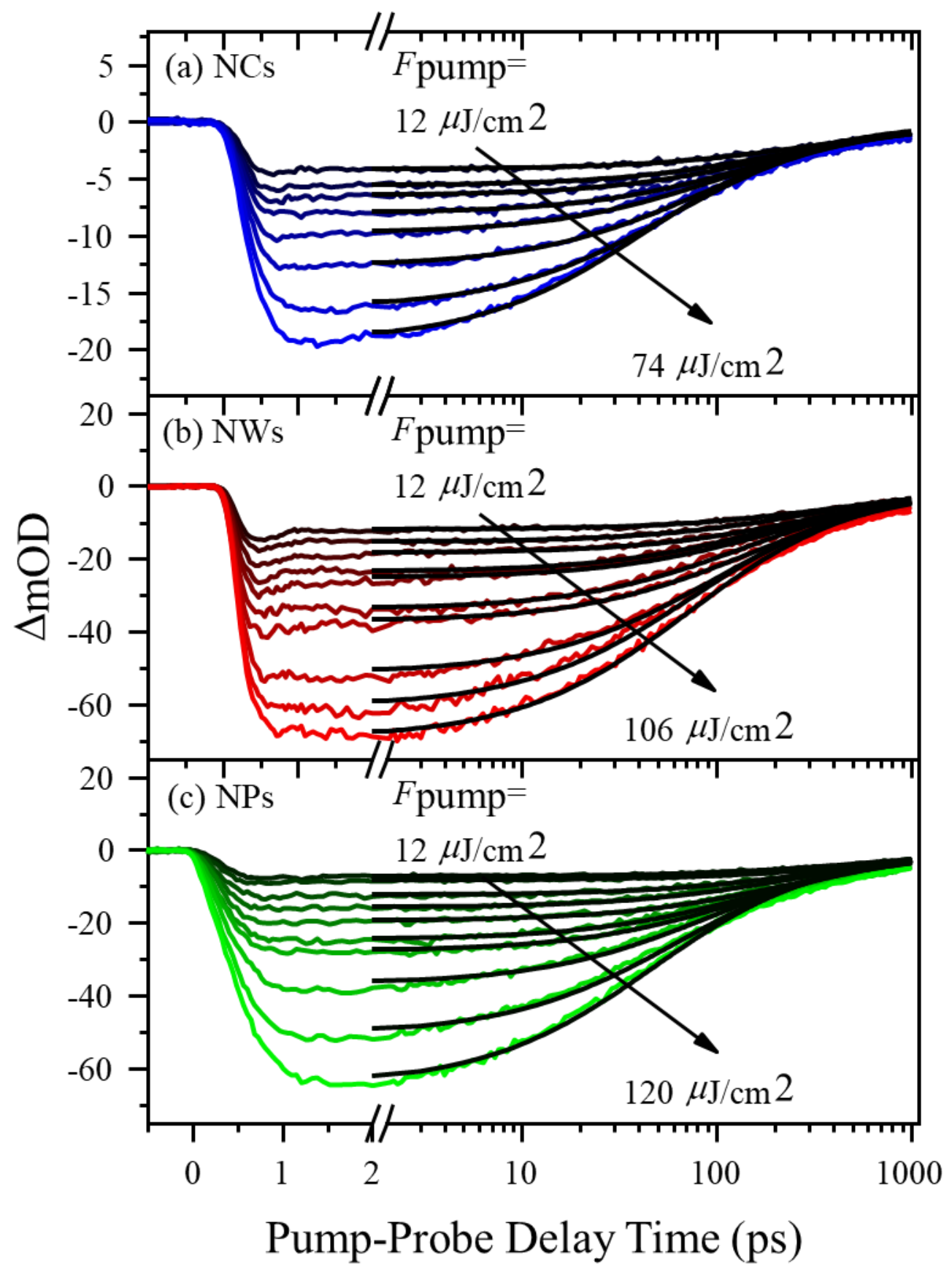

Figure 3.10. Transient kinetics of (a) NCs, (b) NWs, and (c) NPs at hv 2 under different pump fluences. Thin lines are fittings to Equation 3.1. 


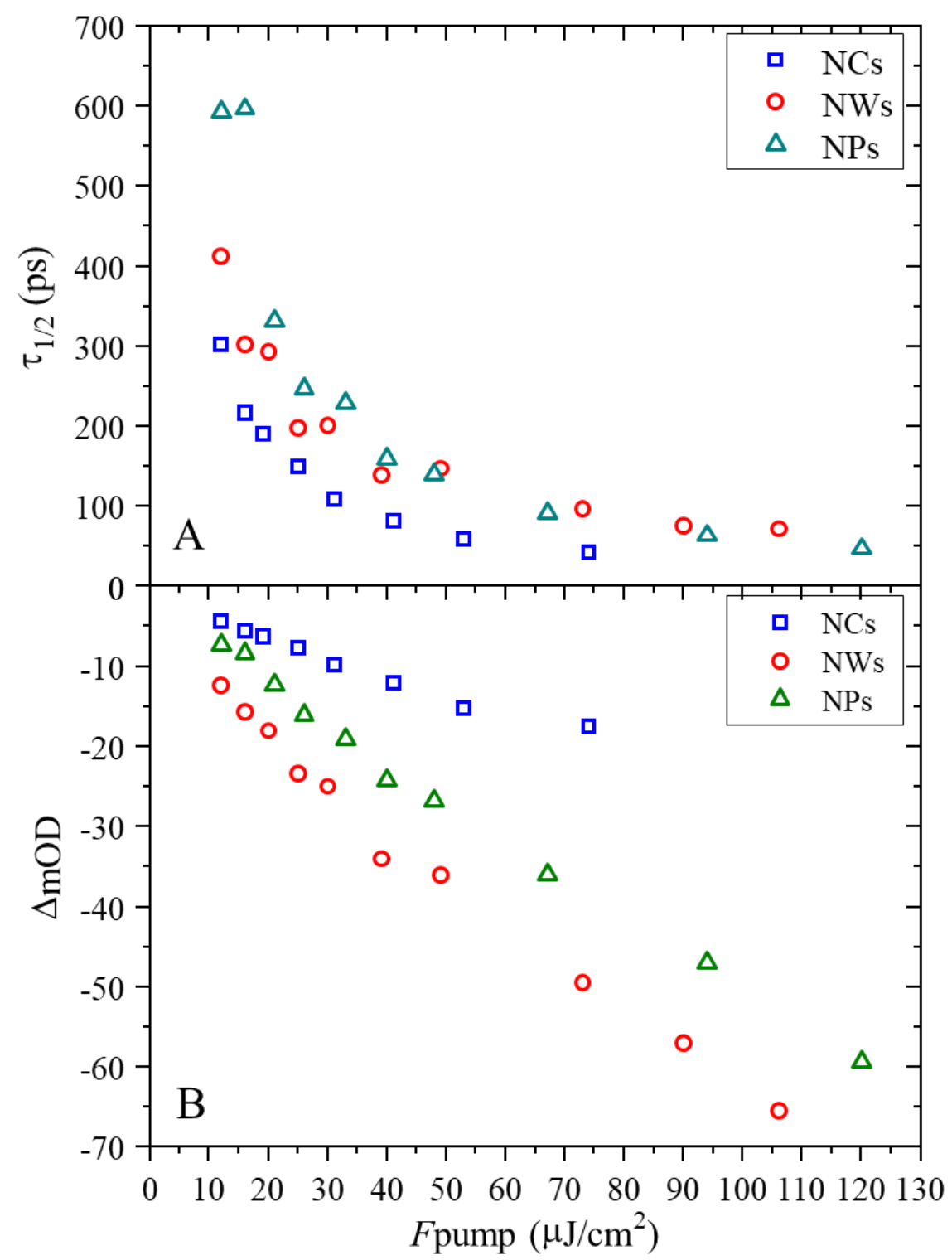

Figure 3.11. (a) Half-time of charge carrier recombination $\left(\tau_{1 / 2, r}\right)$ and $(b)$ maximum SF signal at hv2 under different pump fluences $\left(\mathrm{F}_{\text {pump }}\right)$ for NCs (blue squares), NWs (red circles) and NPs (green triangles). 
For all three nanostructures, a linear relationship between $\mathrm{k}_{\mathrm{r}}$ and $\left[\mathrm{n}_{0}\right]$ was determined. However, the sensitivity of the rate constant $k_{r}$ to the initial charge carrier density $\left[\mathrm{n}_{0}\right]$ is different between the nanostructures. The recombination rate of NWs is independent of $\left[n_{0}\right]$, while those of NCs and NPs vary according to $\left[\mathrm{n}_{0}\right]$. Comparing between the latter two, $\mathrm{k}_{\mathrm{r}}$ of the NCs shows a significantly stronger dependence on $\left[n_{0}\right]$. The effective rate-constant $k_{r}$ can be written as:

$$
k_{r}=k_{r}^{(0)}+\chi_{r}\left[n_{0}\right]
$$

where $k_{r}^{(0)}$ is the effective rate constant at the limit of zero charge carrier, and $\mathrm{Xr}_{\mathrm{r}}$ is the linearity coefficient. and $\mathrm{Xr}$ of all three perovskite nanostructures are listed in Table 3.3.

Table 3.3. Charge carrier recombination rate constants at the zero-density limit $\left(\boldsymbol{k}_{\boldsymbol{r}}^{(\mathbf{0})}\right)$ and linearity coefficients $\mathrm{Xr}$ of $\mathrm{CH}_{3} \mathrm{NH}_{3} \mathrm{PbBr}_{3}$ perovskite nanostructures determined in fitting the transient kinetics.

Nanostructure

\begin{tabular}{cc}
$k_{r}^{(0)}$ & $\mathrm{Xr}$ \\
$\left(10^{-21} \mathrm{~cm}^{3} \mathrm{ps}^{-1}\right)$ & $\left(10^{-30} \mathrm{~cm}^{6} \mathrm{ps}^{-1}\right)$ \\
\hline $2.21(10)$ & $3.07(31)$ \\
\hline $1.81(4)$ & $0^{\mathrm{a}}$ \\
\hline $1.30(6)$ & $0.84(14)$
\end{tabular}

a. Fixed. 


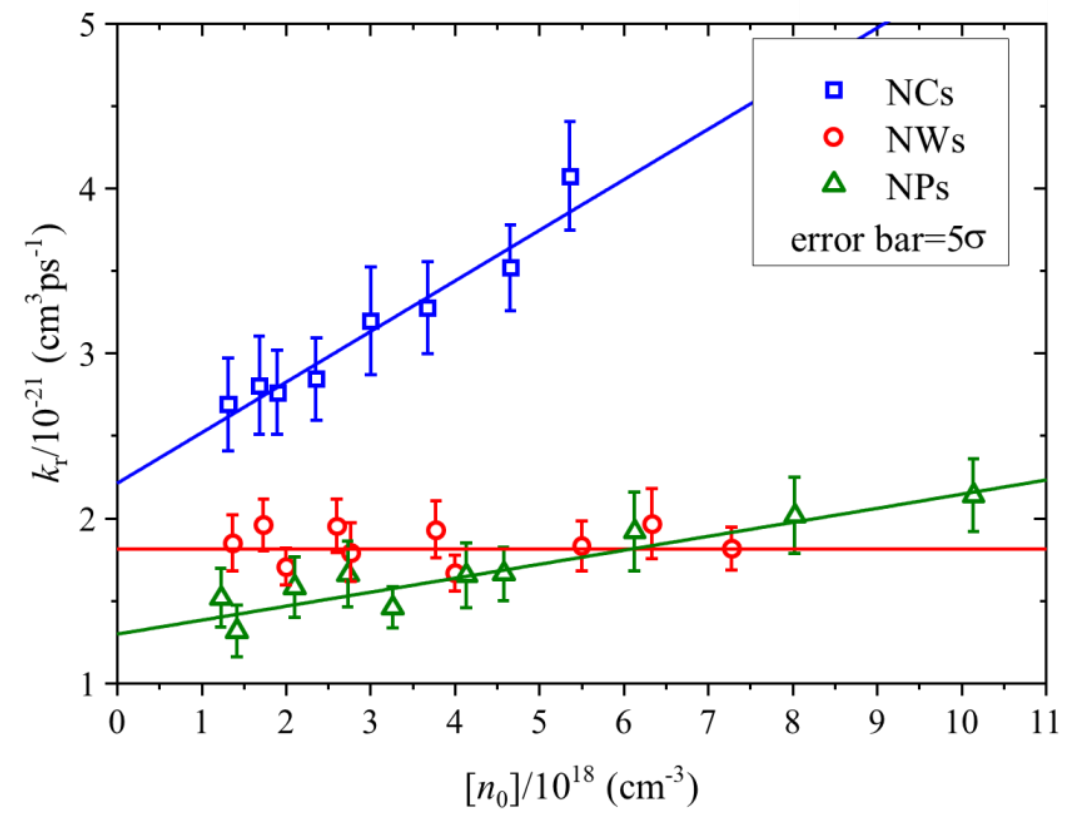

Figure 3.12. Effective charge carrier recombination rate constant $\left(\mathrm{k}_{\mathrm{r}}\right)$ for NCs (blue squares), NWs (red circles) and NPs (green triangles) at different initial charge carrier density $\left[\mathrm{n}_{0}\right]$. Error bars represent five times standard deviation ( $\mathrm{rms}$ ) of fitting the decay of SF/SE signal at $\mathrm{hv}_{2}$ to Equation 3.1. Solid lines are fittings to Equation 3.3.

It is well known that quantum confinement affects charge carrier recombination. In previous works on Auger recombination in CdSe quantum dots, ${ }^{140-141}$ it was observed that the relaxation time of electron-hole pairs demonstrates a cubic size dependence, i.e., is proportional to the volume of the quantum dots. Our volumes of the nanostructures are fixed while the initial charge carrier density is tuned by changing the pump fluence. As in previous works on CdSe quantum dots, the density dependence of charge carrier recombination rate constant $\mathrm{k}_{\mathrm{r}}$ in the perovskite nanostructures is attributed 
to quantum confinement effect even though the mechanism of recombination is different (non-geminate recombination vs. Auger recombination). In nanostructures, electron and hole wave functions are forced to overlap, which reduces dielectric screening and increases the reaction rate. The significant difference between $\mathrm{Xr}$ of different nanostructures can, therefore, be explained by different sizes.

\subsection{The role of trap states}

Trap states, when they exist, may significantly affect both the steadystate and ultrafast spectra. In general, nanostructures have better surface quality owing to the presence of surface ligands. Recently, 3D and 2D lead iodide perovskites, ${ }^{16}$ weak and broad sub-band-gap negative signal has been observed in the TA spectra and attributed to SF of trap states. It was found that the growth of the trap-state SF signal is delayed from the band-edge SF signal by a relaxation time of $\sim 0.5 \mathrm{ps}$. It was also reported that the excitonic traps were enhanced in the quantum-confined 2D NPs. No such trap-state SF signal was observed in our TA spectra. Another experimental evidence for the absence of substantial trap state effects in our perovskite nanostructures comes from the PL spectra. Trap states cause asymmetry of the PL peak, ${ }^{16}$ whereas PL spectra of the perovskite structures are symmetric. On the other hand, if trap states exist, the fluorescence lifetimes should show very long lifetimes. However, lifetimes measured for our samples and found to be in the 100s ns, See figure 3.13 . 

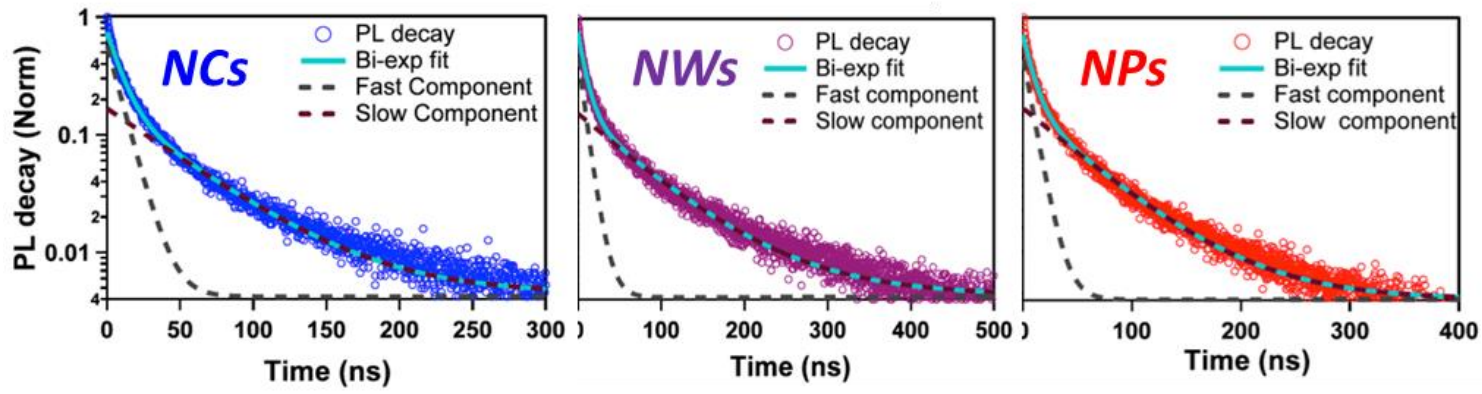

Figure 3.13. Fluorescence life times for the three nanostructures, decay fitting results in a nano-second decay lifetime, which means there is no trap states in the system. Figure was reproduced from reference [49] with permission.

\subsection{Global fitting}

It is possible to simulate the TA spectra in both the frequency and the time domains using a global model, taking into account the photoinduced processes discussed in preceding sub-sections:

$$
\Delta O D(h v, \Delta t)=\left\{\begin{array}{c}
\sum_{i=1,3} A_{i}\left(h v_{i}\right) e^{-\frac{\Delta t}{\tau_{c}}}+ \\
\sum_{i=2,4} A_{i}\left(h v_{i}\right)\left[-e^{-\frac{\Delta t}{\tau_{c}}}+\frac{1}{1+\frac{\Delta t}{\tau_{1 / 2, r}}}\right]
\end{array}\right\} \otimes \operatorname{IRF}(\Delta t)
$$

The first term in the curly brackets describes the decay of the subpicosecond positive $\left(a t h v_{1}\right)$ and negative $\left(\mathrm{at} \mathrm{hv}_{3}\right)$ components. The second term describes the growth and decay of the long-lasting negative $\left(a t h v_{2}\right)$ and positive $\left(\mathrm{at} \mathrm{hv}_{4}\right)$ components. The growth time constant of the long-lasting components is fixed to the decay time constant of the sub-picosecond components (тc). 
Kinetics of the long-lasting components follows that of the intermediate in a consecutive two-step reaction with a first-order reaction preceding a second-order one. The exact solution to the differential rate equations that govern this type of reaction is complicated Fortunately, because the growth time $\left(\tau_{c}\right)$ is more than two orders of magnitude shorter than the decay time $\left(\tau_{1 / 2, r}\right)$, one can approximate the accurate solution with summation of the exact solutions to the first- and the second-order reactions, which are the first and the second terms in the squared brackets, respectively.

The TA kinetics is convolved with IRF:

$$
I R F(t)=\frac{Y_{I R F}}{\sigma_{I R F} \sqrt{2 \pi}} e^{-\frac{1}{2}\left(\frac{t}{\sigma_{I R F}}\right)^{2}}
$$

Where $\sigma=150 \mathrm{fs}$ (corresponding to a FWHM of $355 \mathrm{fs}$ ) is fixed in the global fitting. It has been found that the Lorentzian line shape best reproduces the TA spectra in the frequency domain:

$$
A_{i}(h v)=\frac{\frac{A_{i} \gamma_{i}}{(2 \pi)}}{\left(\frac{\gamma_{i}}{2}\right)^{2}+\left(h v-h v_{i}\right)^{2}}
$$

where $v_{\mathrm{i}}$ is the center frequency, and $\gamma_{\mathrm{i}}$ is the full width at half maximum (FWHM) of the line shape. It has been employed to fit the TA spectra of all three nanostructures under $20 \mu \mathrm{J} / \mathrm{cm}^{2}$ pump fluence $\left(\left[\mathrm{n}_{0}\right] \sim 2 \times 10^{18} \mathrm{~cm}^{-3}\right)$.

The resulted fit parameters are listed in Table 3.4. The Global fits of transient kinetics are shown in Figure 3.4, while figure 3.14 shows the global 
fitting on the frequency domain. In both frequency and the time domains, the experimentally obtained results are well reproduced, which substantiates the spectroscopic and dynamic model.

Radiative $\left(\tau_{r}\right)$ and non-radiative $\left(\tau_{n r}\right)$ lifetimes were calculated from the measured PL lifetimes $\left(\tau_{\mathrm{PL}}\right)$ and quantum yields $\left(\Phi_{\mathrm{FL}}\right)$. In brief, both $\tau_{\mathrm{PL}}$ and $\Phi_{\mathrm{FL}}$ are determined by radiative and non-radiative channels so that: $\frac{1}{\tau_{P L}}=\frac{1}{\tau_{r}}+\frac{1}{\tau_{n r}}$ and $\Phi_{F L}=$ $\frac{\tau_{n r}}{\tau_{r}+\tau_{n r}}$. Therefore, $\tau_{\mathrm{r}}$ and $\tau_{\mathrm{nr}}$ can be calculated using the experimentally determined values of TPL and $\Phi_{\mathrm{FL}}$.

Table 3.4. Peak centers (hv), linewidths $(\gamma),{ }^{\mathrm{a}}$ and time constants of charge carrier cooling $\left(\tau_{c}\right)$ and recombination $\left(\tau_{1 / 2, \mathrm{r}}\right)$ processes determined in the global fittings of TA spectra of the perovskite nanostructures.

\begin{tabular}{ccccccccccc} 
Nanostructure & $h v_{1}$ & $\gamma_{1}$ & $h v_{2}$ & $\gamma_{2}$ & $h v_{3}$ & $\gamma_{3}$ & $h v_{4}$ & $\gamma_{4}$ & $\tau_{\mathrm{c}}$ & $\tau_{1 / 2, \mathrm{r}}$ \\
& $(\mathrm{eV})$ & $(\mathrm{eV})$ & $(\mathrm{eV})$ & $(\mathrm{eV})$ & $(\mathrm{eV})$ & $(\mathrm{eV})$ & $(\mathrm{eV})$ & $(\mathrm{eV})$ & $(\mathrm{ps})$ & $(\mathrm{ps})$ \\
\hline NCs & 2.4 & 0.1 & 2.5 & 0.1 & 2.5 & 0.1 & 2.6 & 0.2 & 0.3 & 139 \\
\hline NWs & 2.4 & 0.1 & 2.4 & 0.1 & 2.4 & 0.1 & 2.5 & 0.2 & 0.2 & 264 \\
\hline NPs & 2.4 & 0.2 & 2.5 & 0.2 & 2.5 & 0.2 & 2.6 & 0.2 & 0.3 & 318 \\
\hline \hline
\end{tabular}

a. $\gamma_{1}, \gamma_{2}$, and $\gamma_{3}$ are fixed to the same values in the global fit. 

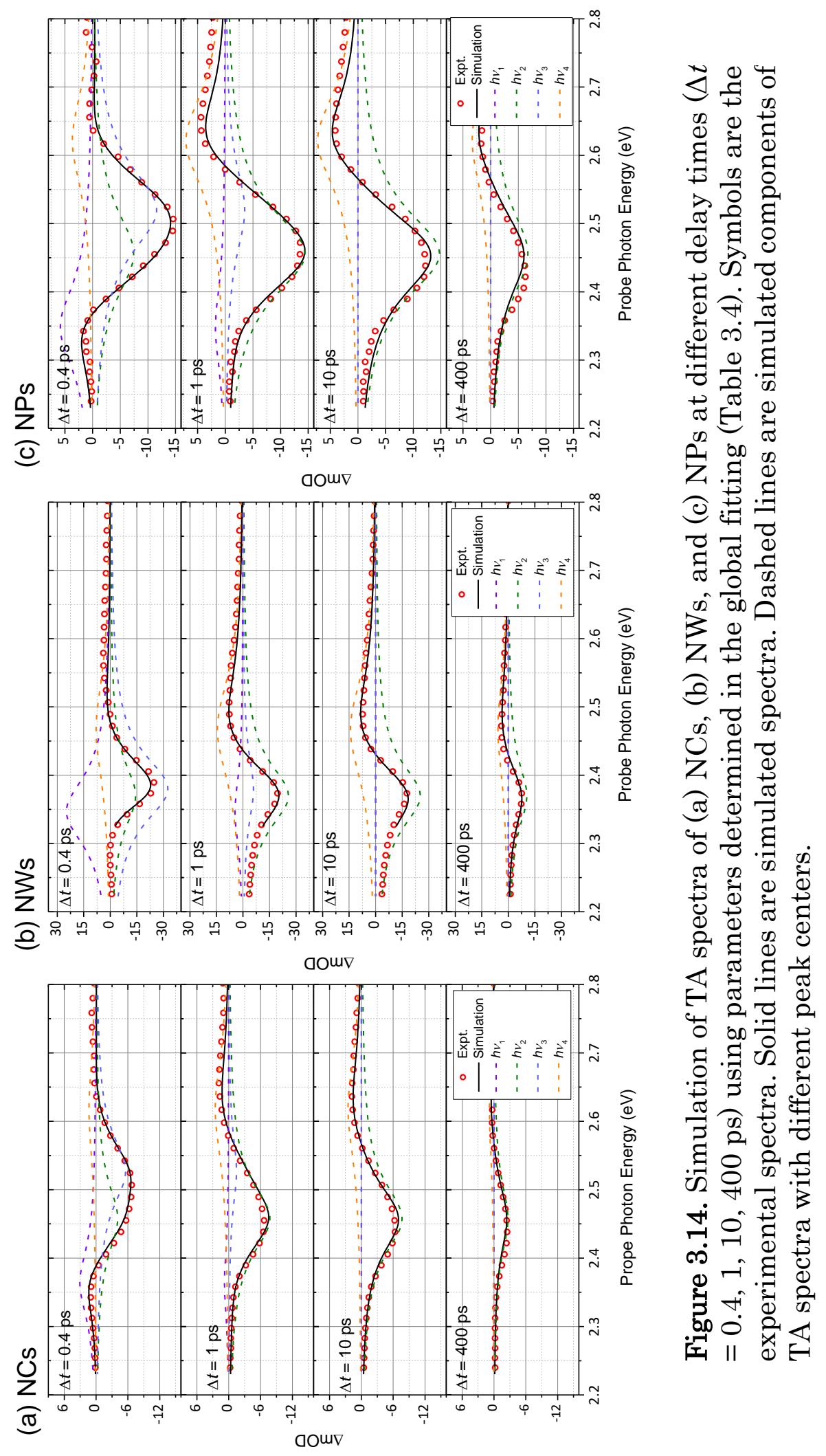


\subsection{Conclusions}

TA spectra of three $\mathrm{CH}_{3} \mathrm{NH}_{3} \mathrm{PbBr}_{3}$ perovskite nanostructures under different pump fluences have been obtained and analyzed. The TA spectra consist of four main features: (1) a sub-band-gap positive PIA signal centered at $\mathrm{hv}_{1}$ induced by BGR; (2) a negative signal at $\mathrm{hv}_{2}$ due to band-edge SF; (3) a blue-shifted SF signal at $\mathrm{hv}_{3}$ owing to the quasi-equilibrium following charge carrier thermalization; and (4) a positive intraband absorption signal centered at $\mathrm{hv}_{4}$. For NCs and NPs, SE also contributes to the negative signal at hv2, whereas for NWs, SE signal is insignificant due to higher carrier density threshold.

Simulation and fitting of the transient kinetics provided a quantitative description of the photoinduced processes. It has been revealed in the ultrafast spectroscopy investigations that photoexcitation in the interband continuum is followed by an instantaneous charge carrier thermalization to a quasiequilibrium distribution, as evidenced by the blue-shifted SF peak that appears within the IRF (FWHM $350 \mathrm{fs}$ ). This SF signal is accompanied by a sub-band-gap PIA signal attributed to photoexcitation-induced transient BGR. The subsequent cooling of hot charge carriers occurs at a sub-picosecond time scale $\left(\tau_{c} \sim 300 \mathrm{fs}\right)$ and is responsible for the decay of the blue-shifted SF and the PIA signals, as well as the growth of a new longer-lasting SF signal, a closelying SE signal (in the TA spectra of $\mathrm{NCs}$ and $\mathrm{NPs}$ ), and an intraband absorption signal in the blue region. The ensuing decay of the SF/SE and the 
intraband absorption signals follows second-order reaction kinetics, which suggests that the observed charge recombination is dominated by the nongeminate recombination process.

In the pump-fluence-dependence investigations, rate constants of charge recombination in the perovskite nanostructures demonstrate strikingly different dependence on the initial charge carrier density upon photoexcitation. The effective rate constants of the NCs and NPs demonstrate a linear relationship with the initial density of the charge carriers, with the former having a much larger linearity coefficient while that of the NWs remains constant.

Absence of observed monomolecular geminate recombination and dependence of the rate of the bimolecular non-geminate recombination on the charge carrier density are attributed to the formation of strong-coupling polarons and self-trapping of electrons. Both observations were predicted by previous X-ray absorption measurements and DFT calculations. Differences between the nanostructures suggest a strong effect of quantum confinement on the recombination process. With same pump fluence and hence similar charge carrier density, both the TA spectrum (in the frequency domain) and transient kinetic (in the time domain) of NWs are significantly different compared to NCs and NPs due to weaker quantum confinement and less overlap between the electron and hole wave functions. Such size and shape dependences may be utilized for the rational selection of appropriate 
nanomaterials for the fabrication of high-efficiency solar cells and lightemitting diodes. 
CHAPTER 4

DIRECT OBSERVATION OF TETRAHYDROFURANYL

(THF-YL) AND TETRAHYDROPYRANYL (THP-YL)

PEROXY RADICALS, A BIOFUEL COMBUSTION

REACTION INTERMEDIATES, VIA CAVITY RING-

\section{DOWN SPECTROSCOPY}

\subsection{Introduction}

Peroxy (ROO•) radicals are one of the most important families of reaction intermediates in low-temperature combustion of fuels. The addition of $\mathrm{O}_{2}$ to a hydrocarbon radical $(\mathrm{R} \bullet)$ forms a peroxy radical and initiates the chain reactions. ${ }^{52-53}$ Peroxy radicals also play a key role in atmospheric chemistry. The initial attack of the hydroxyl radicals $(\cdot \mathrm{OH})$ on hydrocarbons, which may be either biogenic or anthropogenic, and the subsequent addition of oxygen lead to the production of peroxy radicals. In the atmosphere, peroxy radicals are converted to the alkoxy radicals $(\mathrm{RO} \bullet)$ mainly via reaction with NO. $54-55$ 
As alternatives to fossil fuels or as fuel additives, their advantages include renewability and reduced climatic impact. ${ }^{142}$ In contrast to traditional petroleum-based fuels, most biofuels are oxygenated, containing oxygen as an additional element in their molecular structure. Specifically, prototypical cyclic ethers, including tetrahydrofuran (THF) and tetrahydropyran (THP), are important structural building blocks for lignocellulosic biofuels. Therefore, the study of the combustion chemistry of THF and THP, as well as other oxygenated biofuels, has significant scientific and societal importance.

Compared to cyclic alkanes such as cyclopentane and cyclohexane, the combustion chemistry of THF and THP are complicated by several thermodynamic features. Dissociation energies of $\mathrm{C}-\mathrm{O}$ bonds in the tetrahydrofuranyl (THF-yl) and tetrahydropyranyl (THP-yl) radicals are weaker than C-C bands in the cyclopentyl and cyclohexyl radicals. THF-yl and THP-yl are, therefore, susceptible to ring-opening. The oxygen atom also weakens the adjacent $\mathrm{C}-\mathrm{H}$ bonds causing the $\mathrm{B}$-scission pathways to have lower barriers than corresponding cyclic alkanes. Low-temperature oxidation of THF and THP has been studied in combined experimental and computational investigations. ${ }^{20,143-144}$ Mass spectra and photoionization efficiency spectra of many intermediates and products in $\mathrm{THF}^{143}$ and $\mathrm{THP}^{20}$ combustion have been reported. Recently, oxygen addition reaction of the THF-yl radical was investigated by monitoring time-resolved infrared absorption of the $\cdot \mathrm{OH}$ and $\mathrm{HO}_{2} \bullet$ radicals. ${ }^{144}$ However, many unanswered questions invite further 
spectroscopic and kinetic studies. In particular, none of the combustion reaction intermediates of THF and THP have been observed with spectral resolution. Consequentially, no conformer-selective kinetic measurements have been performed.

In this chapter, the laser spectroscopic investigations of the tetrahydrofuranyl peroxy (THFOO•) and tetrahydropyranyl peroxy (THPOO•) radicals will be reported, important reaction intermediates in the oxidation of THF and THP, respectively. The cavity ring-down (CRD) technique ${ }^{145}$ was employed for the direct detection of these peroxy radicals. Aided by quantum chemical calculations, spectral simulation of partially resolved vibronic structure in the experimentally obtained CRD spectra allows identification of molecular carriers of observed transitions.

\subsection{Experimental}

The $\widetilde{\mathrm{A}} \leftarrow \widetilde{\mathrm{X}}$ electronic transitions of THFOO• and THPOO• in the near IR region of 1.15-1.55 $\mathrm{\mu m}$ were recorded by CRD spectroscopy. Absorbance recorded with the photolysis laser off, i.e., absorbance due to precursors, was subtracted from that with the photolysis laser on to obtain net absorbance due to the produced radicals. Kinetic measurements at observed spectral peaks were also performed. In kinetic measurements, the delay time between the photolysis laser pulse and the CRD laser pulse was varied, and absorption was recorded as a function of the delay time. 
The CRD spectroscopy apparatus is described in chapter 2 of in details. For Radical generation via photolysis, ArF excimer laser (Lambda Physik, LPX 120i) was used to provide the $193 \mathrm{~nm}$ photons for photolysis. Also, the fourth harmonic of an Nd:YAG laser (Spectra-Physics, Quanta-Ray Pro-270, $\lambda=266$ $\mathrm{nm}$ ) was used as the photolysis source. A set of diverging and converging cylindrical lenses was used to reshape the photolysis laser beam cross section to a $12 \times 0.5 \mathrm{~cm}^{2}$ rectangle. This increases the photolysis efficiency and the absorption length of the produced radicals. Fluences of both 266 and $193 \mathrm{~nm}$ photolysis lasers were $\sim 15 \mathrm{~mJ} / \mathrm{cm}^{2}$. The spectra were obtained with $193 \mathrm{~nm}$ and $266 \mathrm{~nm}$ photolysis.

The stainless-steel reaction cell has two $20 \mathrm{~cm}$ long rectangular UVgrade quartz side windows for the photolysis laser beam and two $15 \mathrm{~cm}$ long arms on both sides. CRD mirrors (Los Gatos Research, $\mathrm{R}>$ 99.99\%) are mounted on the arms to form a ring-down cavity. The reaction cell was connected to a vacuum pump with a needle valve to control the pumping speed and total pressure in the reaction cell. CRD mirrors and quartz windows were purged by $\mathrm{N}_{2}$ flow continuously to prevent contamination.

Precursors (THF or THP) and $(\mathrm{COCl})_{2}$ were stored in stainless steel reservoirs. $\mathrm{N}_{2}$ flew above the liquid samples and carried their vapors into the stainless-steel reaction cell. Saturation vapor pressures were controlled and stabilized by immersing both precursors and $(\mathrm{COCl})_{2}$ reservoirs in a 1:1 waterethylene glycol bath $\left(-10^{\circ} \mathrm{C}\right)$. A gas delivery system with mass flow controllers 
(MKS, 247D and GE50A) was used to control the flow rates $\left[(\mathrm{COCl})_{2}\right.$ in $\mathrm{N}_{2}$ : 200-300 sccm; precursors in $\left.\mathrm{N}_{2}: 200-300 \mathrm{sccm} ; \mathrm{O}_{2}: 1000-1500 \mathrm{sccm}\right]$. The total pressure in the reaction cell was $\sim 150$ Torr, while partial pressure of the $\mathrm{N}_{2}$ purge gas was controlled by a needle valve to $\sim 50$ Torr. Nitrogen also acted as a third body to remove excess energy from the nascent radicals.

The second harmonic generation of an Nd:YAG laser (Spectra-Physics, Quanta-Ray Pro-270) was used to pump a tunable dye laser (Sirah, PrecisionScan). The pyrromethene and DCM dyes were used to cover the wavelength range of $\sim 585$ to $\sim 670 \mathrm{~nm}$. The visible output from the dye laser (pulse energy 90 mJ) was focused at the center of the $\mathrm{H}_{2}$ cell $(\mathrm{l}=93 \mathrm{~cm}, \mathrm{P}=$ 220 psi) to generate near-IR radiation by Raman shifting. The fundamental, the anti-Stokes, and the first Stokes radiations were blocked by two long-pass filters $($ cutoffs $=780$ and $1000 \mathrm{~nm})$. The transmitted second Stokes radiation $(\Lambda=1.15-1.55 \mu \mathrm{m})$ was collimated by a telescope consisting of two confocal lenses $(f=7.5$ and $2.5 \mathrm{~cm}$ ). A $100 \mu \mathrm{m}$ diameter pinhole was placed at the focal point for mode selection. The resulting second Stokes radiation (1-2 mJ) was directed through the reaction cell and used as the CRD laser to excite the $\tilde{A} \leftarrow$ $\tilde{X}$ transition of the peroxy radicals. The linewidth of the visible fundamental from the dye laser was measured to be $0.06 \mathrm{~cm}^{-1}$ using a spectrometer (HighFinesse, WS-7). The linewidth of the near-IR radiation is limited by the pressure and Doppler broadenings in the $\mathrm{H}_{2}$ cell to about $1 \mathrm{GHz}$. 
Transmission of the CRD laser was focused on and detected by an InGaAs amplified photodiode (Thorlabs, PDA10CS). The signal was acquired with an oscilloscope (Tektronix, DPO3014). The empty-cavity ring-down time $\left(\tau_{0}\right)$ was $\sim 150 \mu$ s. Typically, the ring-down curves were averaged 32 times at each laser wavelength before being sent to a PC via a USB port. A home-built LabVIEW program was used for data processing. The averaged ring-down curves of up to 100,000 data points were fit to a bi-exponential decay function. The longer of the two fit time constants was used to calculate the fractional absorption (in $\mathrm{ppm} / \mathrm{pass}$ ). If necessary, ring-down curves sent to the $\mathrm{PC}$ and/or determined ring-down times at each wavelength were further averaged by the LabVIEW program. Ring-down signal was collected at each wavelength with the excimer photolysis laser on and off. The LabVIEW program controlled the on/off of the excimer laser and wavelength scan of the dye laser, while the experimental apparatus was synchronized by a pulse delay generator (Stanford Research System, DG645) at a $20 \mathrm{~Hz}$ repetition rate.

The kinetic measurements were done by parking the dye laser wavelength at the absorption peak and photolysis-CRD probe delay was varied using the delay generator.

THFOO• and THPOO• radicals were produced by Cl-initiated oxidation of THF and THP. Chlorine atoms were produced by $193 \mathrm{~nm}$ or $266 \mathrm{~nm}$ photolysis of oxalyl chloride ${ }^{146}(\mathrm{COCl})_{2}$ : 


$$
(\mathrm{COCl})_{2} \stackrel{\mathrm{h} v(\lambda=266 \text { or } 193 \mathrm{~nm})}{\longrightarrow} 2 \mathrm{CO}+\mathrm{Cl} \cdot
$$

THF-yl and THP-yl radicals were produced via hydrogen abstraction of corresponding precursors (THF or THP) by chlorine attack,

$$
\mathrm{Cl} \bullet+\mathrm{R}-\mathrm{H} \rightarrow \mathrm{R} \bullet+\mathrm{HCl}
$$

Reaction (3.8) was followed by oxygen addition to form the peroxy radicals:

$$
\mathrm{R} \bullet+\mathrm{O}_{2} \rightarrow \mathrm{ROO} \bullet
$$

\subsection{Conformational search}

The heterocyclic nature of the target radicals leads to a complicated conformational structure. Conformational behaviors of both five ${ }^{147-150}$ and sixmembered rings ${ }^{150}$ have been studied extensively. Fewer studies have been done on the conformational behaviors of heterocyclic rings, including THF and THP. In the case of THFOO• and THPOO• peroxy radicals, the addition of the dioxygen moiety leads to conformational landscapes with labyrinthine complexity.

The conformational search was done as follows. First, conformational structures of the ring were taken into account. Then the regioisomers were determined according to different positions for the addition of the dioxygen moiety on the ring with respect to the ring oxygen atom. Next, possible 
orientations of the peroxy group with respect to the ring (axial and equatorial) were considered.

Finally, three possible orientations of the $\mathrm{OOCH}$ dihedral angle were included: gauche with the terminal peroxy oxygen towards the ring oxygen $(G)$, trans $(\mathrm{T})$, and gauche with the terminal oxygen away from the ring oxygen (G').

\subsubsection{THFOO• Conformers}

Conformational behavior of THF is a subject of extensive experimental and computational studies. It has been generally accepted that THF has two optically distinguishable stable conformers, namely, the bent and twisted structures, although there are disagreements on their relative energies. ${ }^{151-153}$ The bent and twisted structures are derivatives from the "envelope" and the "half-chair" ring structures of cyclopentane, respectively. The former conformer of THF belongs to the $\mathrm{C}_{2}$ point group, while the latter belongs to the $\mathrm{C}_{\mathrm{s}}$ point group. They are close in energy and connected by pseudo-rotation with low barriers $\left(<100 \mathrm{~cm}^{-1}\right)$. Therefore, these two conformers have comparable populations at room temperature.

For both the bent and twisted conformations, there exist two unique positions on the ring for the $\mathrm{O}_{2}$ addition, namely, $a$ and $B$, with respect to the oxygen atom. When the two possible orientations of the peroxy group (axial and equatorial) and the three possible $\mathrm{OOCH}$ dihedral angles are also taken into account, there are $2 \times 2 \times 2 \times 3=24$ initial geometries for THFOO . 


\subsubsection{THPOO• Conformers}

THP has 38 basic conformations: two chairs, six boats, six twistedboats, twelve half-chairs, and twelve envelopes. ${ }^{154-155}$ The two chairs are equivalent to each other. They have significantly lower energy than the other conformers and are separated from them by high barriers $(\sim 10 \mathrm{kcal} / \mathrm{mol}) .{ }^{155-156}$ For THPOO•, conformers with the chair ring structure are $\sim 6 \mathrm{kcal} / \mathrm{mol}$ lower in energy than the other stable conformers, which have the twist-boat ring structure. Since the barrier is considered high at current experimental conditions, other ring conformers are not expected to be populated under our conditions. However, the possibility of presenting the twisted boat conformers was tested via quantum chemical calculations and found that these conformers have high energies, and they are not expected to be populated at room temperature. Hence, we will only observe chair conformers. See section (Quantum Chemical calculation) for relative energy calculations.

There are three unique positions with respect to the oxygen atom on the six-membered ring of THP ( $\alpha, B$, and $\gamma$ ). For a or B-THPOO• regioisomers, six optically distinguishable conformers are possible because the peroxy group can have axial or equatorial orientations and the $\mathrm{OOCH}$ dihedral angle can be $\mathrm{G}$, T, or G'. For the $\gamma$-THPOO regioisomer, there are only four optically distinguishable conformers because the $\mathrm{G}$ and G' conformers are mirror images 
of each other. In total, there are $6+6+4=16$ initial structures. They are named similar to THFOO•

\subsection{Quantum Chemical calculations}

No quantum chemical calculations on these two radicals have been reported yet, to the best of our knowledge. However, a comprehensive survey of the conformational landscapes of THFOO• and THPOO• was not attempted. Instead, the goals of the computational investigation were to determine the lowest-energy stable conformers, to optimize their geometries, and to calculate their molecular constants for spectral simulation.

Density functional theory (DFT) calculations for geometry optimization and vibrational frequencies of ground-state THFOO and THPOO• conformers were carried out at the B3LYP/6-31+G(d) level of theory using Gaussian 09 software package. ${ }^{157}$ The Complete Basis Set (CBS)-QB3 composite method was also employed to predict the relative energies of the conformers. The results are close to those determined in DFT calculations. Time-dependent (TD)DFT calculations at the same level were employed for calculations of excited-state molecules. Both the vertical and adiabatic excitation energies for the $\tilde{A} \leftarrow \tilde{X}$ transition were determined from the calculations. The Calculations results are summarized in Table 4.1 for THFOO• and Table 4.2 for THPOO•. Also, vibronic transitions Franck-Condon factors (FCF) were calculated using 
the ezSpectrum program. ${ }^{158}$ Comparison of DFT and CBS-QB3 are listed in tables 4.3 and 4.4 for THFOO and THPOO, respectively.

Table 4.1. Calculated relative energies, Boltzmann distribution factors (at $300 \mathrm{~K})$, relative populations, and the adiabatic $\tilde{A}-\tilde{X}$ excitation energies ( $\left.\Delta E^{\tilde{A}-\tilde{X}}\right)$ for the lowest-energy conformers of THFOO•. Boltzmann distribution factors are normalized for each Regio isomer. Population Ratio for $\alpha$ and $B$ was set to $82.5-17.5$.

\begin{tabular}{lccccc} 
Conformer & $\begin{array}{c}\text { No. of } \\
\text { enantiomers }\end{array}$ & $\begin{array}{c}\text { relative } \\
\text { energy } \\
\left(\mathbf{c m}^{-1}\right)\end{array}$ & $\begin{array}{c}\text { Boltzmann } \\
\text { distribution } \\
\text { factor }\end{array}$ & $\begin{array}{c}\text { Relative } \\
\text { population } \\
(\%)\end{array}$ & $\begin{array}{c}\Delta E^{\widetilde{A}-\widetilde{X}} \\
\left(\mathbf{c m}^{-1}\right)\end{array}$ \\
\hline a-Ax-T & 2 & 345 & 0.06 & 5.3 & 7129 \\
\hline a-Ax-G & 2 & 122 & 0.22 & 17.8 & 7134 \\
\hline a-Ax-G' & 2 & 0 & 0.42 & 34.6 & 7648 \\
\hline a-Eq-T & 2 & 370 & 0.06 & 4.6 & 7086 \\
\hline a-Eq-G & 2 & 195 & 0.15 & 12.0 & 7070 \\
\hline a-Eq-G' & 2 & 267 & 0.10 & 8.1 & 7380 \\
\hline B-Ax-T & 2 & 2560 & 0.01 & 0.2 & 7418 \\
\hline B-Ax-G & 2 & 2001 & 0.29 & 5.0 & 7717 \\
\hline B-Ax-G' & 2 & 2154 & 0.12 & 2.2 & 7610 \\
\hline B-Eq-T & 2 & 2331 & 0.05 & 0.8 & 7638 \\
\hline B-Eq-G & 2 & 2023 & 0.25 & 4.5 & 7658 \\
\hline B-Eq-G' & 2 & 2010 & 0.27 & 4.8 & 7523 \\
\hline \hline
\end{tabular}


Table 4.2. Calculated relative energies, Boltzmann distribution factors (at $300 \mathrm{~K})$, relative populations, and the adiabatic $\tilde{A}-\tilde{X}$ excitation energies ( $\left.\Delta E^{\tilde{A}-\tilde{X}}\right)$ for the lowest-energy conformers of THPOO• Boltzmann distribution factors are normalized for each Regio isomer. Population Ratio for $\alpha$ and $B$ and $\gamma$ was set to $0: 2: 1$.

\begin{tabular}{lccccc} 
Conformer & $\begin{array}{c}\text { No. of } \\
\text { enantiomers }\end{array}$ & $\begin{array}{c}\text { relative } \\
\text { energy } \\
\left(\mathbf{c m}^{-1}\right)\end{array}$ & $\begin{array}{c}\text { Boltzmann } \\
\text { distribution } \\
\text { factor }\end{array}$ & $\begin{array}{c}\text { Relative } \\
\text { population } \\
\text { (\%) }\end{array}$ & $\begin{array}{c}\Delta \boldsymbol{E}^{\widetilde{A}-\widetilde{X}} \\
\left(\mathbf{c m}^{-1}\right)\end{array}$ \\
\hline $\mathrm{a}-\mathrm{Ax}-\mathrm{T}$ & 2 & 707 & 0.02 & 0 & 6402 \\
\hline $\mathrm{a}-\mathrm{Ax}-\mathrm{G}$ & 2 & 30 & 0.42 & 0 & 6749 \\
\hline $\mathrm{a}-\mathrm{Ax}-\mathrm{G}^{\prime}$ & 2 & 0 & 0.53 & 0 & 7362 \\
\hline $\mathrm{a}-\mathrm{Eq}-\mathrm{T}$ & 2 & 1106 & 0.00 & 0 & 7165 \\
\hline $\mathrm{a}-\mathrm{Eq}-\mathrm{G}$ & 2 & 707 & 0.02 & 0 & 7138 \\
\hline $\mathrm{a}-\mathrm{Eq}-\mathrm{G}^{\prime}$ & 2 & 921 & 0.01 & 0 & 7837 \\
\hline B-Ax-T & 2 & 3001 & - & - & - \\
\hline B-Ax-G & 2 & 1584 & 0.20 & 13.1 & 7615 \\
\hline B-Ax-G' & 2 & 1637 & 0.15 & 10.1 & 7512 \\
\hline B-Eq-T & 2 & 1634 & 0.13 & 8.4 & 7498 \\
\hline B-Eq-G & 2 & 1443 & 0.35 & 23.1 & 7422 \\
\hline B-Eq-G' & 2 & 1587 & 0.18 & 12.1 & 7417 \\
\hline$\gamma-\mathrm{Ax}-\mathrm{T}$ & 1 & 2127 & 0.00 & 0.1 & 6940 \\
\hline$\gamma-\mathrm{Ax}-\mathrm{G}$ & 2 & 1205 & 0.26 & 8.7 & 7431 \\
\hline$\gamma-\mathrm{Eq}-\mathrm{T}$ & 1 & 1283 & 0.09 & 2.9 & 7604 \\
\hline$\gamma-\mathrm{Eq}-\mathrm{G}$ & 2 & 1029 & 0.65 & 21.7 & 7513 \\
\hline \hline (a) Tran & 2 & & & & \\
\hline
\end{tabular}

(a) Transition state. 
Table 4.3. Relative energies, Boltzmann factors, and relative populations of THFOO - conformers calculated at the B3LYP/6-31+G(d) level of theory and using the CBS-QB3 composite method.

\begin{tabular}{ccccccc}
$\begin{array}{c}\text { THFOO- } \\
\text { Conformers }\end{array}$ & $\begin{array}{c}\text { Relative energy } \\
\left(\mathrm{cm}^{-1}\right)\end{array}$ & \multicolumn{2}{c}{$\begin{array}{c}\text { Boltzmann } \\
\text { distribution factor }\end{array}$} & $\begin{array}{c}\text { Relative } \\
\text { population } \\
\text { (\%) }\end{array}$ \\
& CBS-QB3 & B3LYP & CBS-QB33 & B3LYP & CBS-QB3 & B3LYP \\
\hline$a-$ Ax-T & 293 & 345 & 0.11 & 0.06 & 9.4 & 5.3 \\
\hline$\alpha-\mathrm{Ax}-\mathrm{G}$ & 201 & 122 & 0.18 & 0.22 & 14.6 & 17.8 \\
\hline$a-\mathrm{Ax}-\mathrm{G}^{\prime}$ & 0 & 0 & 0.46 & 0.42 & 38.2 & 34.6 \\
\hline$a-\mathrm{Eq}-\mathrm{T}$ & 369 & 370 & 0.08 & 0.06 & 6.5 & 4.6 \\
\hline$\alpha-\mathrm{Eq}-\mathrm{G}$ & 324 & 195 & 0.1 & 0.15 & 8.1 & 12 \\
\hline$a-\mathrm{Eq}-\mathrm{G}^{\prime}$ & 396 & 267 & 0.07 & 0.1 & 5.7 & 8.1 \\
\hline$b-\mathrm{Ax}-\mathrm{T}$ & 2954 & 2560 & 0.02 & 0.01 & 0.3 & 0.2 \\
\hline$b-\mathrm{Ax}-\mathrm{G}$ & 2411 & 2001 & 0.23 & 0.29 & 4.1 & 5 \\
\hline$b-\mathrm{Ax}-\mathrm{G}^{\prime}$ & 2583 & 2154 & 0.1 & 0.12 & 1.8 & 2.2 \\
\hline$b-\mathrm{Eq}-\mathrm{T}$ & 2568 & 2331 & 0.11 & 0.05 & 1.9 & 0.8 \\
\hline$b-\mathrm{Eq}-\mathrm{G}$ & 2396 & 2023 & 0.25 & 0.25 & 4.4 & 4.5 \\
\hline$b-\mathrm{Eq}-\mathrm{G}^{\prime}$ & 2364 & 2010 & 0.29 & 0.27 & 5.1 & 4.8 \\
\hline \hline & & & & & & \\
\hline
\end{tabular}


Table 4.4. Relative energies, Boltzmann factors, and relative populations of THPOO $B$ and $\gamma$ conformers calculated at the B3LYP/6-31+G(d) level of theory and using the CBS-QB3 composite method.

\begin{tabular}{ccccccc}
$\begin{array}{c}\text { THPOO- } \\
\text { Conformers }\end{array}$ & $\begin{array}{c}\text { Relative energy } \\
\left(\mathbf{c m}^{-1}\right)\end{array}$ & \multicolumn{2}{c}{$\begin{array}{c}\text { Boltzmann } \\
\text { distribution factor }\end{array}$} & $\begin{array}{c}\text { Relative population } \\
\text { (\%) }\end{array}$ \\
& CBS-QB3 & B3LYP & CBS-QB3 & B3LYP & CBS-QB3 & B3LYP \\
\hline$b-\mathrm{Ax}-\mathrm{G}$ & 448 & 555 & 0.27 & 0.2 & 18.1 & 13.1 \\
\hline$\beta-\mathrm{Ax}-\mathrm{G}^{\prime}$ & 480 & 608 & 0.23 & 0.15 & 15.4 & 10.1 \\
\hline$b-\mathrm{Eq}-\mathrm{T}$ & 592 & 605 & 0.14 & 0.13 & 9 & 8.4 \\
\hline$b-\mathrm{Eq}-\mathrm{G}$ & 470 & 414 & 0.24 & 0.35 & 16.2 & 23.1 \\
\hline$b-\mathrm{Eq}-\mathrm{G}$ & 620 & 558 & 0.12 & 0.18 & 7.9 & 12.1 \\
\hline$\gamma-\mathrm{Ax}-\mathrm{T}$ & 973 & 1098 & 0 & 0 & 0.1 & 0.1 \\
\hline$\gamma-\mathrm{Ax}-\mathrm{G}$ & 25 & 176 & 0.38 & 0.26 & 12.8 & 8.7 \\
\hline$\gamma-\mathrm{Eq}-\mathrm{T}$ & 186 & 254 & 0.18 & 0.09 & 5.9 & 2.9 \\
\hline$\gamma-\mathrm{Eq}-\mathrm{G}$ & 0 & 0 & 0.43 & 0.65 & 14.5 & 21.7 \\
\hline \hline
\end{tabular}

THFOO •: The addition of the $-\mathrm{OO}$ moiety lowers the symmetry of the molecule so that all conformers of THFOO• belong to the $\mathrm{C} 1$ point group. After performing DFT calculations for the 24 conformers, we found that if two initial geometries have the same $\mathrm{OO}$ position and $\mathrm{OOCH}$ dihedral angle but different ring structures (bent and twisted) and orientation of the peroxy group (axial and equatorial), they converge to enantiomers of each other upon geometry optimization. Figure 4.1 illustrates the 12 resulting optically distinguishable stable conformers of THFOO• They are named according to the position of the 
-OO moiety on the ring ( $\alpha$ or $B$ ), the axial/equatorial orientation of the peroxy group, and the $\mathrm{OOCH}$ dihedral angle.
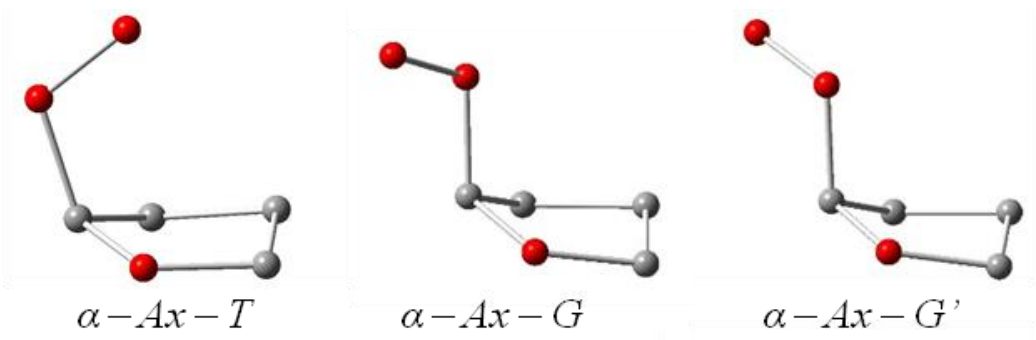

$\alpha-A x-G$

$\alpha-A x-G^{\prime}$
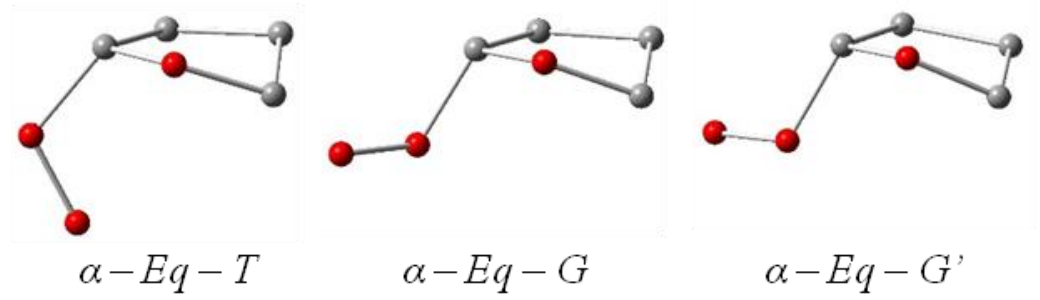

$$
\alpha-E q-G
$$

$\alpha-E q-G^{\prime}$
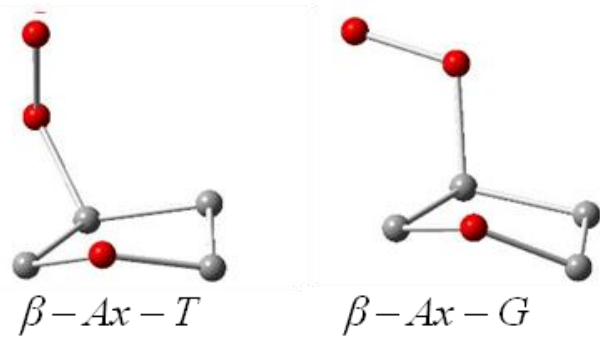

$\beta-A x-G$
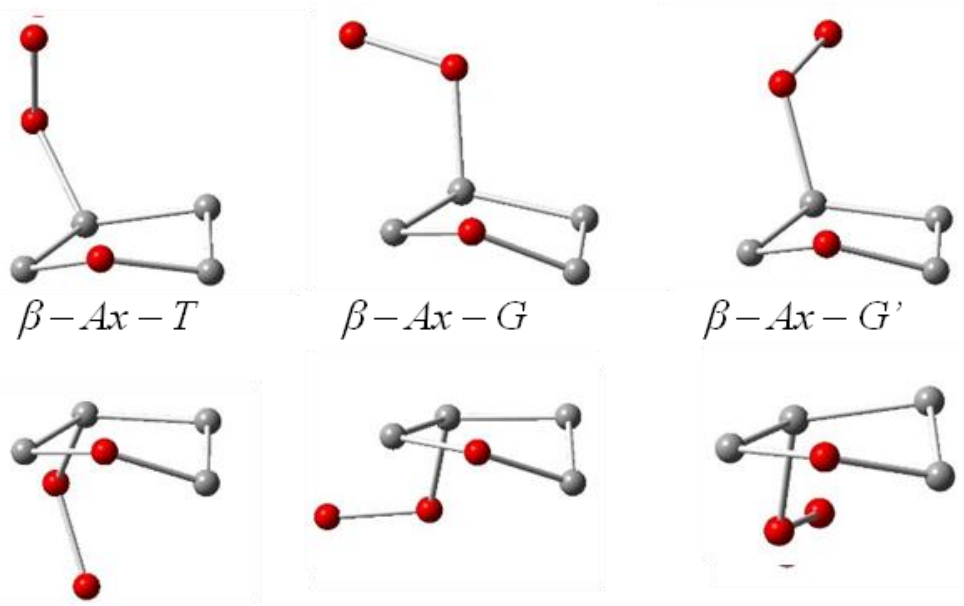

$$
\beta-E q-T
$$

$\beta-E q-G$

$\beta-E q-G^{\prime}$

Figure 4.1. Lowest-energy conformers of the THFOO• radical, ground state geometries optimized by DFT calculations. Hydrogen atoms are omitted for clarity.

THPOO: Since only low-energy conformers have significant concentrations under the current experimental conditions, only chair conformations of THPOO- are expected to present in the reaction cell. 
However, we calculated for all possible chair conformers in addition to twisted boat conformers to make sure they will not contribute to the spectra. The relative energies for twisted conformers are listed in table 4.5. These energies are relative to the lowest-energy conformer of the respective isomer, which has the boat ring structure. Optimized geometries are shown in figure 4.2.

Table 4.5. Relative energies of selected THPOO• conformers with the twist-boat ring structure.

\begin{tabular}{ccc}
$\begin{array}{c}\text { Ring } \\
\text { Conformation }\end{array}$ & Conformer & $\begin{array}{c}\text { Relative Energy a,b } \\
\left(\mathbf{c m}^{-1}\right)\end{array}$ \\
\hline \multirow{2}{*}{ Twist-Boat } & $b-\mathrm{Ax}-\mathrm{G}$, & 2113 \\
\cline { 2 - 3 } & $\beta-\mathrm{Eq}-\mathrm{G}$, & 2092 \\
\cline { 2 - 3 } & $\gamma-\mathrm{Ax}-\mathrm{G}$ & 2312 \\
\cline { 2 - 3 } & $\gamma-\mathrm{Eq}-\mathrm{G}$ & 2007
\end{tabular}

\footnotetext{
a. Relative to the lowest-energy conformer of the respective isomer, which has the boat ring structure.

b. With zero-point-energy corrections.
}

One of the chair conformers, the $\mathrm{B}-\mathrm{Ax}-\mathrm{T}$ structure, converges to a transition state upon geometry optimization, which has an $\mathrm{OOCH}$ dihedral angle of $145.8^{\circ}$. Figure 4.3 shows the PES scan along the OCCH Dihedral angle of the $\mathrm{B}-\mathrm{Ax}$ conformers. This is due to the steric interaction between the ring oxygen and the terminal peroxy oxygen. This transition state connects the B$A x-G$ and the $B-A x-G$ ' conformers. Thus, the total number of optically distinguishable stable conformers is reduced to 15 conformers. Figure 4.4 
illustrates the 15 resulting optically distinguishable stable conformers of THPOO•

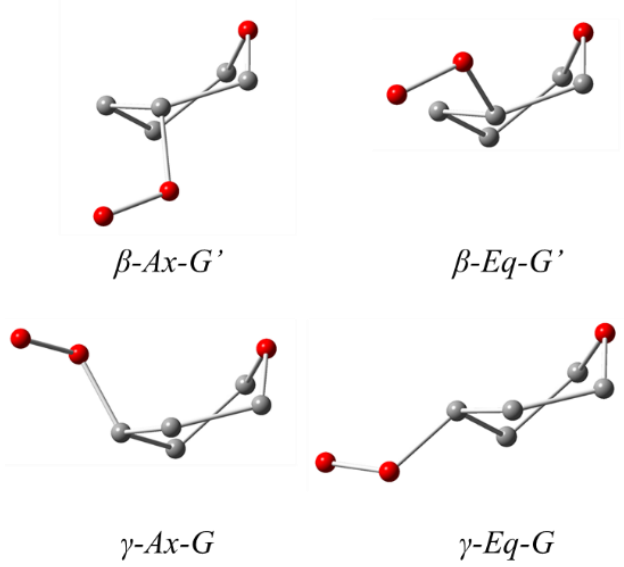

Figure 4.2. Optimized geometries using UB3LYP calculated for twisted boat conformers.

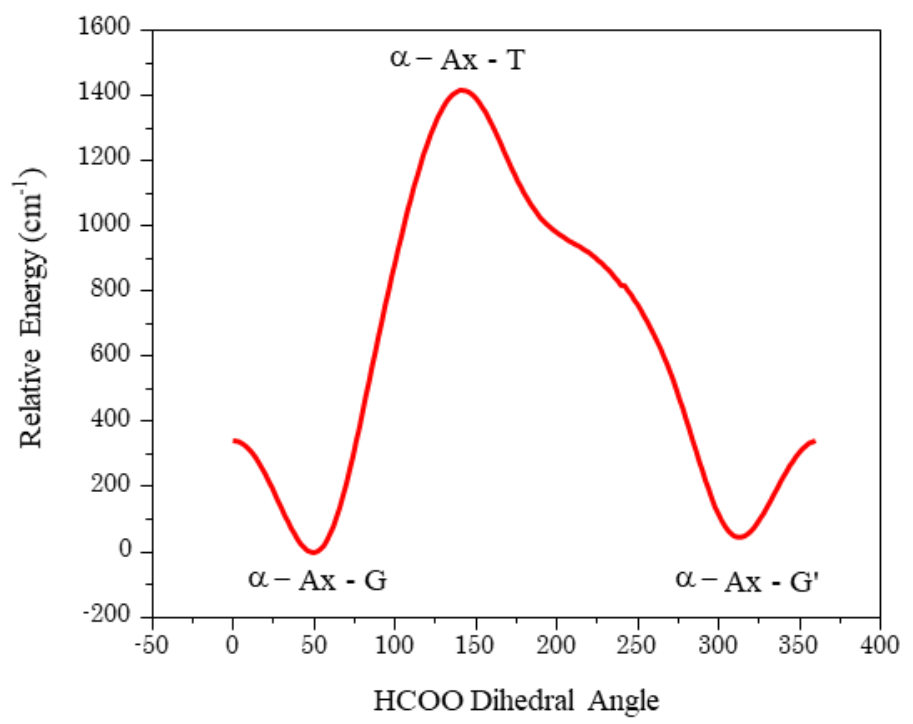

Figure 4.3. PES scan along $\mathrm{OCCH}$ Dihedral angle of the $B-A x$ conformers 

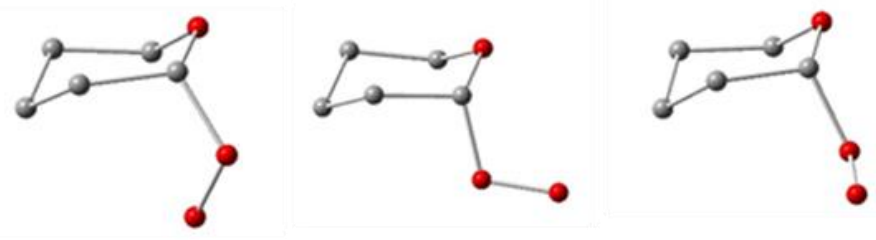

$a-A x-T$

$a-A x-G$

$a-A x-G^{\prime}$
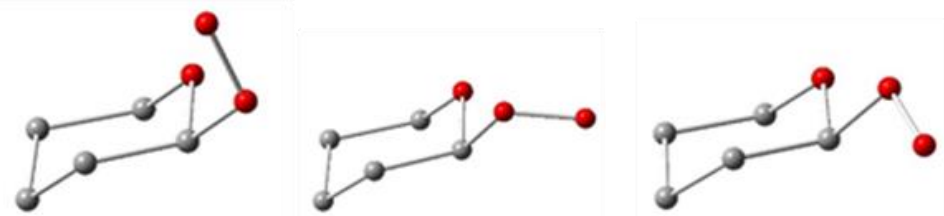

$a-E q-T$

$a-E q-G$

$a-E_{q}-G^{\prime}$
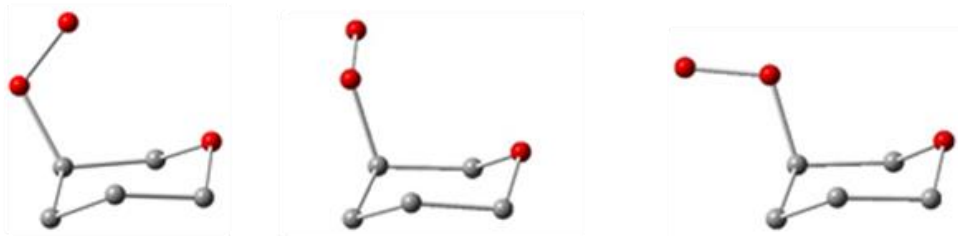

$\beta-A x-T$

$$
\beta-A x-G
$$$$
\beta-A x-G^{\prime}
$$
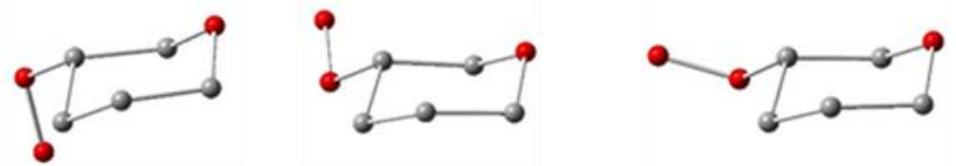

$\beta-E q-T$

$\beta-E q-G$

$\beta-E q-G^{\prime}$

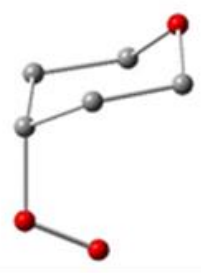

$y-A x-T$

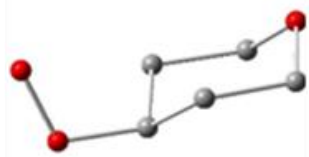

$y-E q-T$

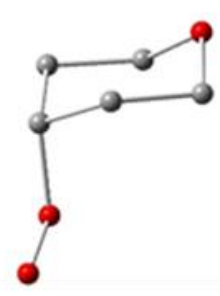

$y-A x-G$

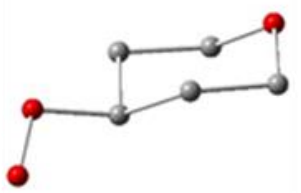

$y-E q-G$

Figure 4.4. Lowest-energy conformers of the THPOO• radical. All such conformers have the "chair" ring structure. ground state geometries optimized by DFT calculations. Hydrogen atoms are omitted for clarity. Note that $B-A x-T$ is a transition state. 


\subsection{Intensity Calculations and Spectral simulation}

\subsubsection{Intensity calculations}

The simulation of experimentally observed spectra uses the calculated adiabatic electronic transition frequencies and vibrational frequencies of each conformer. For intensity calculations, several factors were taken into account:

(1) Population ratio of regioisomers ( $\alpha-$ and $B-$ THFOO $\bullet ;-, \beta-$, and $\gamma-$ THPOO•). If the reaction rate for $\mathrm{O}_{2}$ addition is significantly faster than the lifetimes of the THF-yl, and THP-yl radicals, it is reasonable to assume that the population ratio of different regioisomers of THFOO• or THPOO• is equal to that of THF-yl or THP-yl radicals. This is, in turn, determined by the branching ratio of hydrogen abstraction by the $\mathrm{Cl}$ atom at different positions on the ring. Rotavera et al. ${ }^{20}$ calculated the distribution of initial $a-,, B-$, and $\gamma-\mathrm{THP}-\mathrm{yl}$ radicals produced in the $\mathrm{THP}+\mathrm{Cl} \rightarrow \mathrm{THP}-\mathrm{yl}+\mathrm{HCl}$ reaction using the structure-activity relationship (SAR) ${ }^{159-160}$ on a per-CH bond basis. Their results suggest that $\mathrm{a}-\mathrm{THP}-\mathrm{yl}$ has the greatest branching ratio of $76 \%$, while those of the $8-$ and $\gamma-\mathrm{THP}-\mathrm{yls}$ are $16 \%$ and $8 \%$, respectively. ${ }^{20}$

Adopting the SAR used in Reference 20, relative populations of $\alpha-$ and $8-\mathrm{THF}-$ $\mathrm{yl}$ radicals produced in the $\mathrm{THF}+\mathrm{Cl} \rightarrow \mathrm{THF}-\mathrm{yl}+\mathrm{HCl}$ reaction were calculated to be $82.5 \%$ and $17.5 \%$, respectively. 
(2) Relative populations of different conformers of each regioisomer. Given the relatively high temperature and pressure in the reaction cell, conversion between different low-energy conformers of a certain isomer is estimated to be significantly faster than the timescale of CRD measurements. It is therefore assumed that relative populations of all conformers of each regioisomer follow the Boltzmann distribution.

(3) The number of equivalent structures of each conformer. This is equal to the number of enantiomers for THFOO- and THPOO due to their low symmetry. The $\gamma-\mathrm{Eq}-\mathrm{T}$ and $\gamma-\mathrm{Ax}-\mathrm{T}$ conformers of THPOO belong to the $\mathrm{C}_{\mathrm{s}}$ point group. They each have a single conformation. Other conformers of THPOO• and all conformers of THFOO $\bullet$ belong to the $\mathrm{C}_{1}$ point group and have two enantiomers.

(4) Oscillator strength of the $\widetilde{\mathrm{A}} \leftarrow \widetilde{\mathrm{X}}$ electronic transition for each conformer. This is assumed to be the same for all regioisomers and conformers of THFOO• or THPOO• because the electron promotion is localized to the OO moiety.

(5) FCFs of the vibronic transitions. FCF calculations suggest that only the origin $\left(v^{\prime}=0 \leftarrow v^{\prime \prime}=0\right)$ transitions and transitions to $\tilde{A}$-state vibrational levels with OO-stretch characters have considerable FCFs. 
Relative transition intensities of all conformers of THFOO and THPOO- are calculated as the product of factors listed above. Under the current experimental conditions, the rotational structure of the free radicals cannot be resolved. Instead, rotational contours with a width of about $15 \mathrm{~cm}^{-1}$ were recorded. Therefore, transition intensities are convoluted by a Gaussian line shape with a full width at half maximum (FWHM) of $15 \mathrm{~cm}^{-1}$ to generate the simulated spectra. The simulations of THFOO and THPOO conformers are plotted in figures 4.5 and 4.6 , respectively.
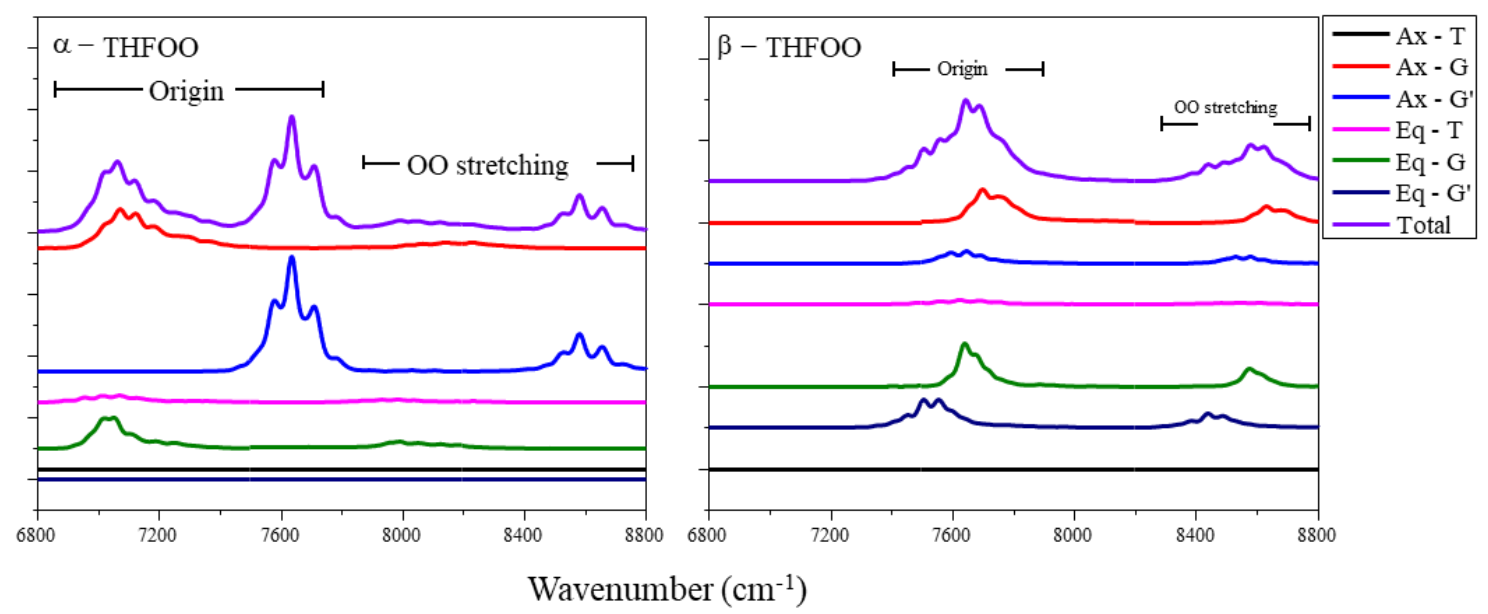

Figure 4.5. Simulated spectra of a (left) and B (right) THFOO conformers. Overall spectrum of is calculated as summation of spectra of all conformers weighted by their relative populations. All spectra are offsetted for clarity. 

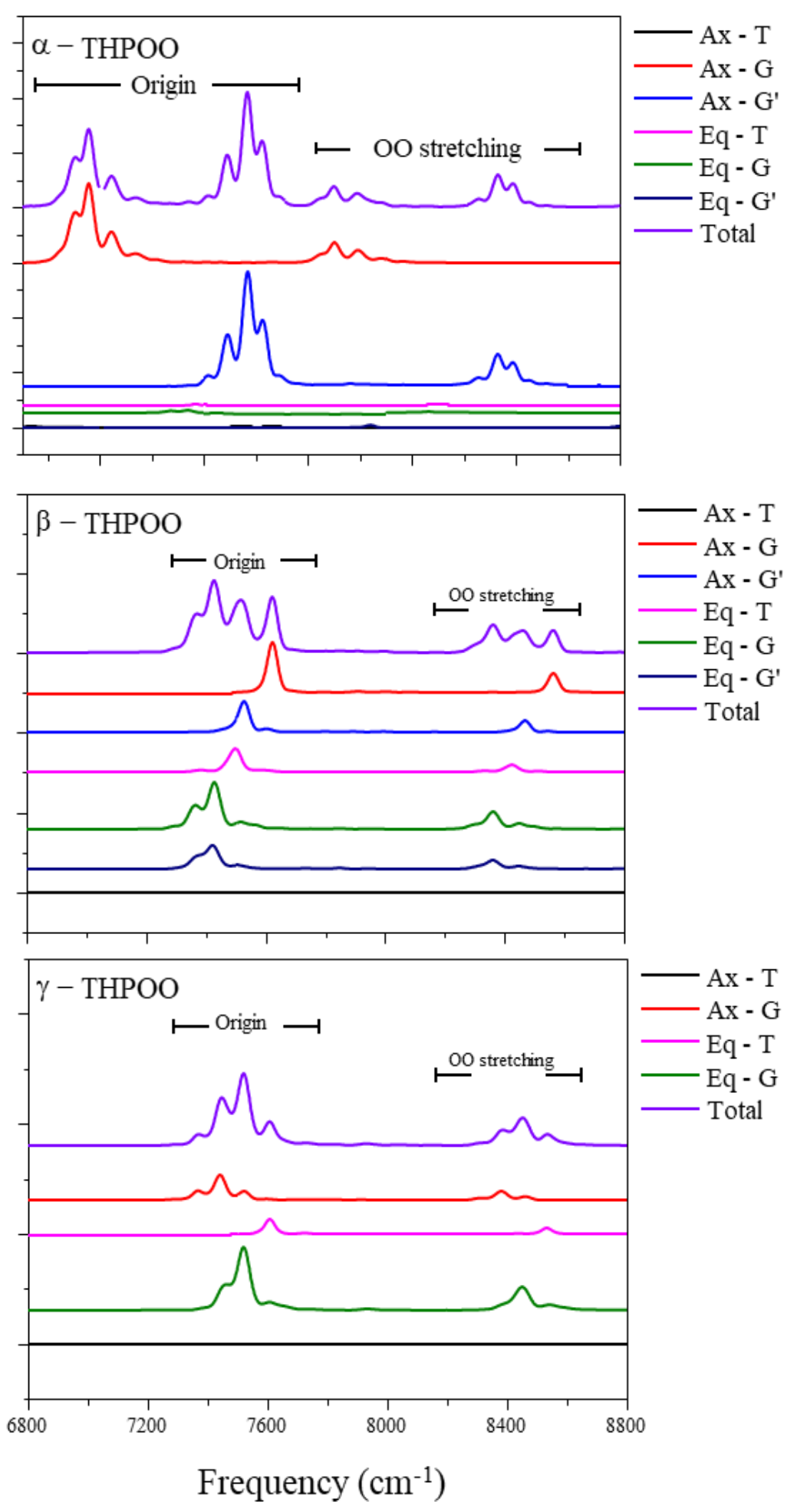

Figure 4.6. Simulated spectra of $\alpha$ (Top) and $\beta$ and $\gamma$ (Bottom) THPOO conformers. Overall spectrum of is calculated as summation of spectra of all conformers weighted by their relative populations. All spectra are offsetted for clarity. 


\subsubsection{Spectral simulations (Python data analysis)}

Since both THFOO and THPOO• peroxy radicals had a large number of conformers, and several calculations and preparations have to be done for each conformer before simulation, which is time-consuming, a python program was written to do all necessary pre-simulation operations. The program consists of three main subprograms. Preinstallation and pre-requirements to use the program are given in appendix $\mathrm{A}$, along with the code and detailed explanation for the script. Figures 4.5 and 4.6 above were obtained using the python program.

\subsection{Results and Discussion}

\subsubsection{Overview of the spectra}

CRD spectra (obtained by using $193 \mathrm{~nm}$ photolysis) of THF and THP precursors are shown in figure $4.7 \mathrm{a}$ and $4.7 \mathrm{~b}$ respectively in comparison with spectra of the peroxy absorption, subtraction of the "photolysis-off" spectra (correspond only for the precursor) from the "photolysis-on" spectra (corresponds to precursor and peroxy radicals) generates the target radicals absorption spectra. In the obtained spectra, for both THFOO• and THPOO•, there are two main groups of peaks, the lower-energy set of peaks (Group A) can be assigned to the origin bands and the higher-energy one (Group B) to the $\mathrm{OO}$ stretch bands, if compared to the simulated spectra. They are centered 
at 7650 and $8600 \mathrm{~cm}^{-1}$ for THFOO•, and 7500 and $8400 \mathrm{~cm}^{-1}$ for THPOO• All peaks in Groups A and B depend on the presence of oxygen and oxalyl chloride in the reaction cell.

Also, another relatively narrow peaks observed in our spectra between 6800-7200 $\mathrm{cm}^{-1}$, labeled $\mathrm{x}, \mathrm{y}$, and $\mathrm{z}$ for THFOO• and $\mathrm{x}, \mathrm{y}$ for THPOO . These peaks were also observed in CRD spectra previously obtained using $\mathrm{Cl}-$ initiated oxidation of cyclopentane and cyclohexane. Although all three peaks depend on the presence of oxygen in the reaction cell, kinetic measurements showed that these peaks have lifetimes of more than $50 \mathrm{~ms}$ (the maximum delay time allowed by our experimental setup). This is significantly longer than the measured lifetimes of $\sim 0.5-10 \mathrm{~ms}$ for peaks in Groups A and B, which are relatively broad and attributed to peroxy radicals. (See Figure 4.8 for measured decay kinetics.) Although a definitive identification of molecular carriers of Peaks $\mathrm{x}, \mathrm{y}$, and $\mathrm{z}$ is not available at this stage, they are attributed to stable molecules rather than free radicals because of the lifetime measurements.

TD-DFT calculations overestimate both the electronic transition frequencies and the $\mathrm{OO}$ stretch frequencies. Only one scaling factor was used for all isomers and conformers of THFOO• or THPOO•, to better simulate the experimental spectra, although its value is different for these two molecules (0.885 for THFOO• and 0.865 for THPOO•). Numerical values of the scaling factors are determined so that the maxima in the simulated spectra match those in the experimental one. A common vibrational scaling factor of 0.920 is 
adopted for the $\mathrm{OO}$ stretch vibrational frequencies of both THFOO and $\mathrm{T}$ $\mathrm{HPOO} \cdot$

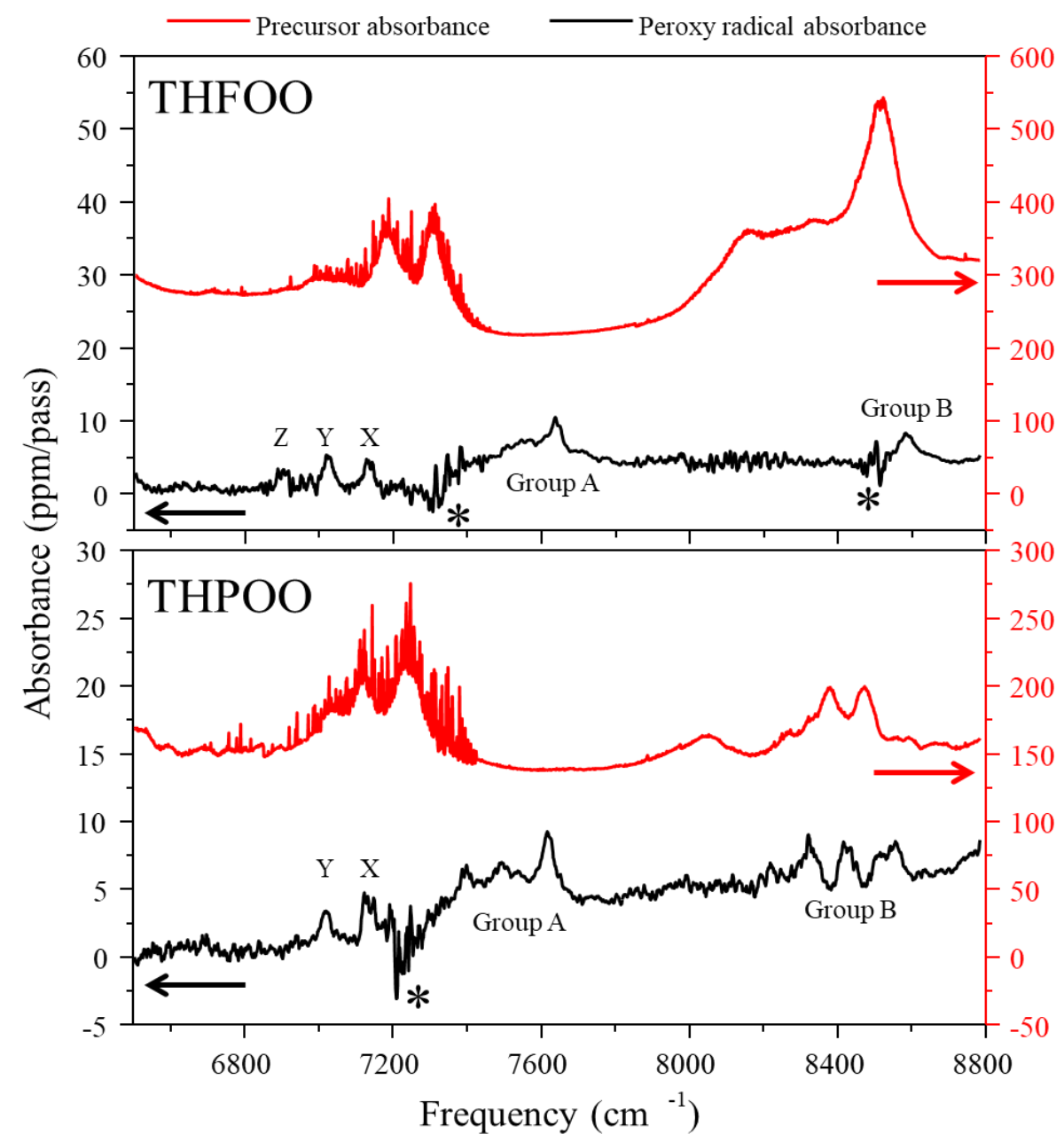

Figure 4.7. CRD spectra of THF (top) and THP (bottom) precursors (red traces) in comparison with those of the THFOO • and THPOO $\cdot$ radicals (black traces), respectively. Radical spectra are offsetted for clarity. Dips labeled with asterisks are due to strong precursor absorption. 


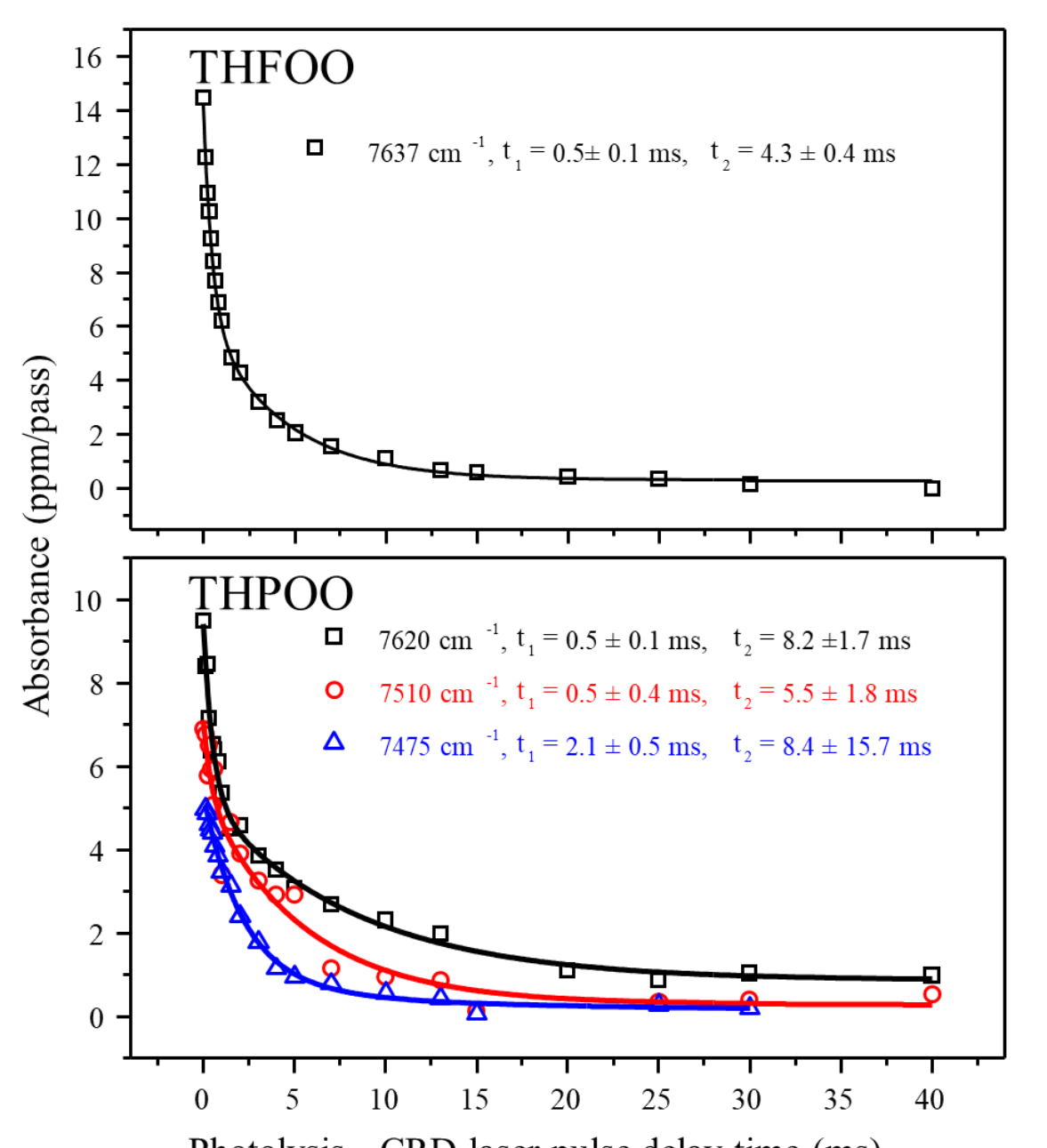

Photolysis - CRD laser pulse delay time (ms)

Figure 4.8. Decay kinetic of THFOO• (top) and THPOO• (bottom) radicals measured at different peaks in their CRD spectra. The decay curves are fit to a bi-exponential function with fit time constants given in the figures.

\subsubsection{Artificial dips in the spectra}

An artificial-dips in the CRD spectra, which are labeled with asterisks in figure 4.7, appears due to strong precursor absorption, where the loss in precursor concentration due to the generation of the peroxy radicals will also be detected by CRDS. 
THP or THF precursor strong absorption contaminates observed transitions of peroxy radicals in the $\mathrm{OO}$ stretch region. For the THPOO•, the signal at $8400 \mathrm{~cm}^{-1}$ is strongly overlapping with the precursor absorption, as seen in figure 4.5. Subtracting the photolysis-off spectrum from the photolysis-on one may introduce negative features (dips) in the net spectrum resulting from depletion of the precursor by photolysis. Hence, this contamination could be just dips from the precursor subtraction rather than a structure of OO stretching, especially the simulation predicts such structure and strong features. However, kinetic measurements were carried out in the OO stretch region to confirm that all strong features vanish within $15 \mathrm{~ms}$ (see Figures 4.8 and 4.9). Therefore, absorption peaks in this region are attributed to free radicals rather than precursor molecules. 


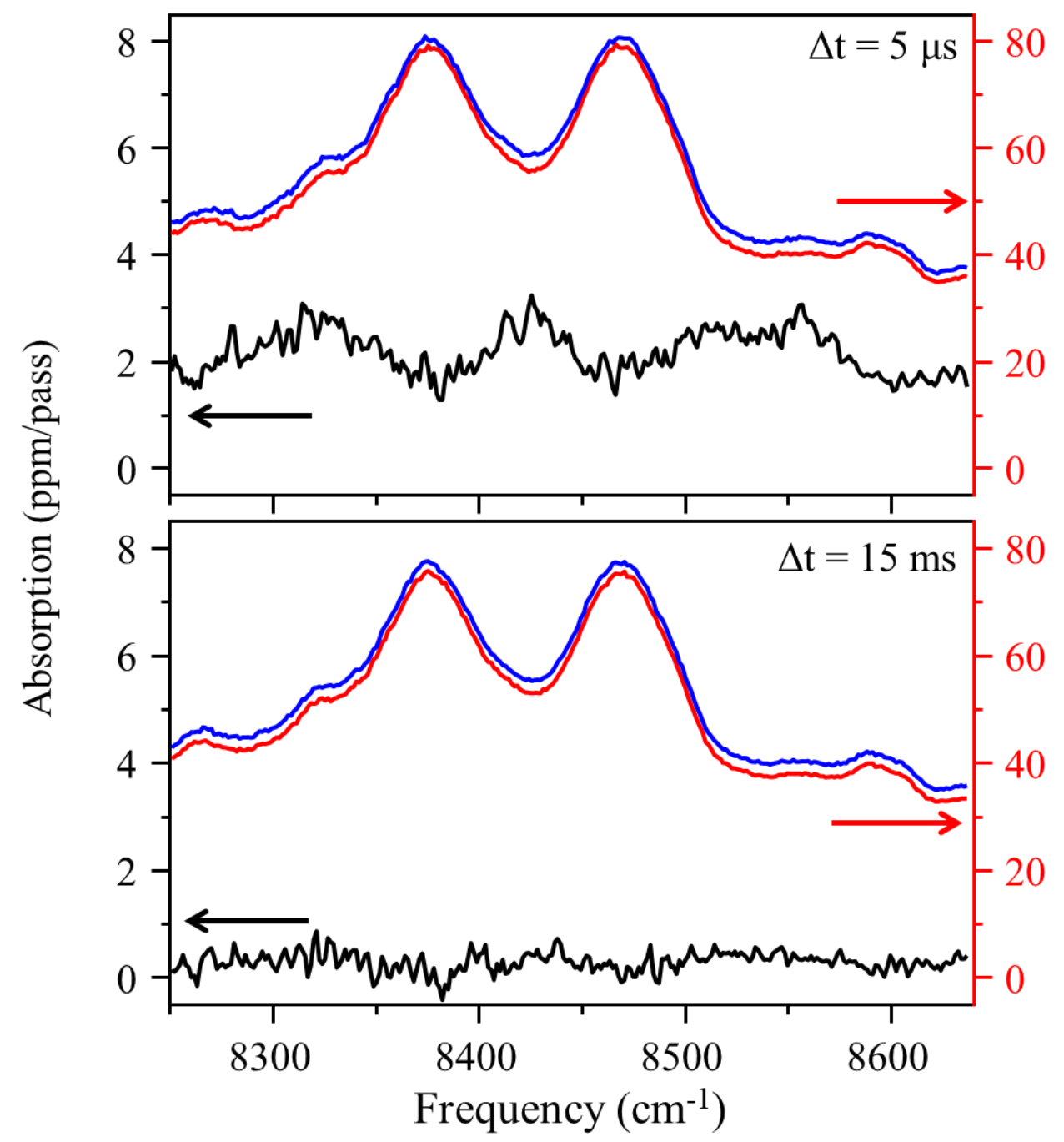

Figure 4.9. CRD spectra recorded with $5 \mu \mathrm{s}$ (top) and $15 \mathrm{~ms}$ (bottom) delay times between the photolysis and the CRD laser pulses $(\Delta \mathrm{t})$. Absorption peaks are assigned to OO stretch of THPOO• radicals.

\subsubsection{THP and THF Absorption cross-section at $193 \mathrm{~nm}$.}

The use of $193 \mathrm{~nm}$ photolysis of Oxalyl Chloride $(\mathrm{COCl})_{2}$ to produce $\mathrm{Cl} \bullet$ atoms that initiate the chemistry. According to the Mainz UV cross-section database, the absorption cross-section of $(\mathrm{COCl})_{2}$ is similar to that of THP and 
THF at $193 \mathrm{~nm}$. Since the concentration of THF and THP is comparable to the concentration of $(\mathrm{COCl})_{2}$ in the reaction mixture, the photolytic products of THF or THP would also be comparable to the $\mathrm{Cl}$ atom production. This means that there are numerous radical products of photolysis of THF and THP at 193 $\mathrm{nm}$, including $\mathrm{H}$, allyl, vinoxy, formyl, hydroxymethyl, methyl, and the ringopened isomers. ${ }^{161-162}$ All of them are reactive and may form other peroxy radicals or react with each other or with stable molecules, i.e., the expected spectra will have a very complex structure. However, to confirm that the observed spectra correspond only for the target radicals, we did use a $266 \mathrm{~nm}$ laser (fourth harmonics of an Nd:YAG laser) as our photolysis source. The recorded CRD spectra in the origin band region with $266 \mathrm{~nm}$ photolysis are shown in figure $4.10 \mathrm{a}$ and $4.10 \mathrm{~b}$ for both THFOO and THFOO•, respectively, in comparison with those obtained using $193 \mathrm{~nm}$ photolysis. As demonstrated in that figure, spectra obtained using $193 \mathrm{~nm}$, and $266 \mathrm{~nm}$ photolysis lasers have almost identical spectral features in terms of frequencies and shapes of the absorption peaks. Eventually, using 193 shows a butter signal to noise ratio.

However, it is possible that radicals other than peroxies were produced in the reaction cell upon $193 \mathrm{~nm}$ photolysis. ${ }^{161-162}$ The fact that we did not observe new peaks using $193 \mathrm{~nm}$ photolysis compared to $266 \mathrm{~nm}$ photolysis suggests that electronic transitions of these free radicals are not in the same wavelength region of THPOO• and THFOO• 


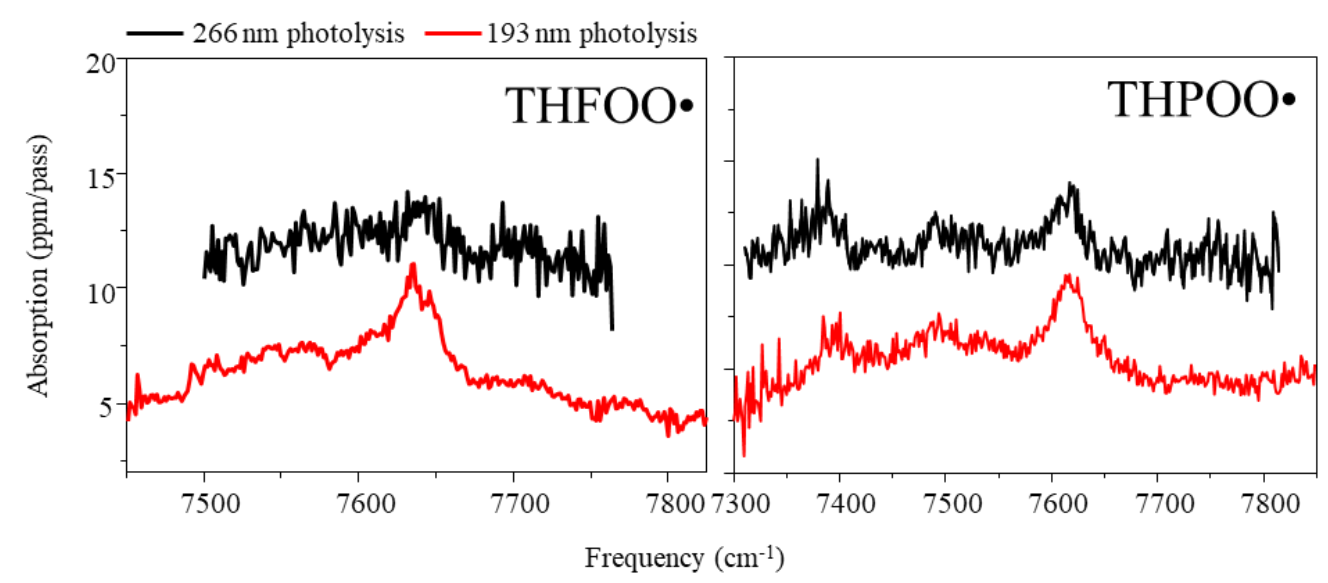

Figure 4.10. CRD spectra obtained by $266 \mathrm{~nm}$ (black) and $193 \mathrm{~nm}$ (red) photolysis of (a) THF and (b) THP in the presence of excessive $\mathrm{O}_{2}$.

On the other hand, peroxy radicals other than THFOO and THPOO• may also be produced in the presence of oxygen, including the methyl peroxy $\left(\mathrm{CH}_{3} \mathrm{OO} \bullet\right)$ and the allyl peroxy $\left(\mathrm{H}_{2} \mathrm{C}=\mathrm{CH}-\mathrm{CH}_{2} \mathrm{OO} \bullet\right)$ radicals. ${ }^{161-162} \tilde{\mathrm{A}} \leftarrow \tilde{\mathrm{X}}$ transitions of these two peroxy radicals have been obtained in previous CRD spectroscopy measurements. The origin band of $\mathrm{CH}_{3} \mathrm{OO} \bullet$ is at $7383 \mathrm{~cm}^{-1}$, while conformers of $\mathrm{H}_{2} \mathrm{C}=\mathrm{CH}-\mathrm{CH}_{2} \mathrm{OO} \bullet$ have strong origin band transitions at 7440 $\mathrm{cm}^{-1}$ and $\sim 7600 \mathrm{~cm}^{-1} .{ }^{163}$ Absorption peaks at these three frequencies was not observed in the CRD spectrum, which confirms that the resulting spectra correspond only to the target radicals.

\subsection{Ring-Opening.}

a-THP-yl is expected to have a fast dissociation rate because of its weak $\mathrm{Ca}^{\prime}-\mathrm{O}$ bond. As a result, the spectra of THPOO- will not be observed. The assumption of decomposition of a-THP-yl is still a topic of debate. 
Unimolecular decomposition rate coefficient for a-THP-yl peroxy was calculated to be about 0.01 per second at $300 \mathrm{~K}$. in other words, the addition of $\mathrm{O}_{2}$ should dominate the decomposition of alpha-THP-yl peroxy. Therefore, this isomer should also dominate the experimental spectrum.

However, similar to a-THP-yl, a-THPOO• is also expected to have a fast dissociation rate because of its weak $\mathrm{Ca}^{\prime}-\mathrm{O}$ bond. The assumption for $\mathrm{a}-$ THP-yl dissociation applies to a-THPOO• as well.

The weakest $\mathrm{CO}$ bond in a-THPOO•, $\mathrm{Ca}^{\prime} \mathrm{O}$, has dissociation energy of only $\sim 10 \mathrm{kcal} / \mathrm{mol}$ (at $0 \mathrm{~K}$ ) calculated using the CBS-QB3 method. This is due to the repulsion between the lone pairs on the ring oxygen atom and the bonding pair in the $\mathrm{Ca}^{\prime} \mathrm{O}$ bond. Electron is withdrawn from the electron-rich $\mathrm{Ca}^{\prime} \mathrm{O}$ bond toward the electron-deficient $\mathrm{CaO}$ bond through the ring oxygen is energetically favorable, and dissociation of the $\mathrm{Ca}^{\prime} \mathrm{O}$ bond forms a stable $\mathrm{Ca}=$ O bond. As a result, a-THPOO regioisomers are susceptible to ring-opening. We, therefore, assume that a-THPOO radicals have lifetimes significantly shorter than the ring-down time $(\sim 150 \mu \mathrm{s})$ as well as the delay time between the photolysis laser pulse and the CRD laser pulse (tens of microseconds) so that they were not detected under our experimental conditions. We further assume that the population ratios of the $\beta-$, and $\gamma$-THPOO radicals are determined by that of the nascent $8-$, and $\gamma-$ THP-yls $(2: 1)$. An attempt of simulation for THPOO radicals was also carried out using a 76:16:8 ratio 
shown in figure 4.11. The predicted peaks in the region of $6600-7000 \mathrm{~cm}^{-1}$ due to the a-regioisomers are absent in the experiment spectrum.

Bond dissociation energies for ring-opening of the THFOO • radical were also calculated using the CBS-QB3 method. The dissociation energy of the weakest $\mathrm{Ca}^{\prime} \mathrm{O}$ bond in $\mathrm{a}-\mathrm{THFOO} \cdot$ regioisomers was found to be $\sim 83 \mathrm{kcal} / \mathrm{mol}$ (at $0 \mathrm{~K}$ ), which is significantly larger than that of the a-THPOO radical $(\sim 10$ $\mathrm{kcal} / \mathrm{mol}$ ). The stronger $\mathrm{Ca}^{\prime} \mathrm{O}$ bond in THFOO - compared to THPOO• is attributed to a $\angle \mathrm{Ca}{ }^{\prime} \mathrm{OCa}$ bond angle that is much closer to that of a tetrahedral polygon $\left(\angle \mathrm{Ca}^{\prime} \mathrm{OCa}=110^{\circ}\right.$ in $\alpha-\mathrm{THFOO} \bullet$ vs. $116^{\circ}$ in $\left.\alpha-\mathrm{THPOO} \bullet\right)$ and, hence, a much smaller angle strain. Ring-opening is, therefore, less likely for THFOO• under the current experimental conditions. Thus, a population ratio of 82.5:17.5 is used in the simulation of the THFOO• spectrum. Bond dissociation energy (BDE) of the lowest-energy conformers of all regioisomers of THPOO• and THFOO• calculated using the CBS-QB3 method are summarized in table 4.6 . 


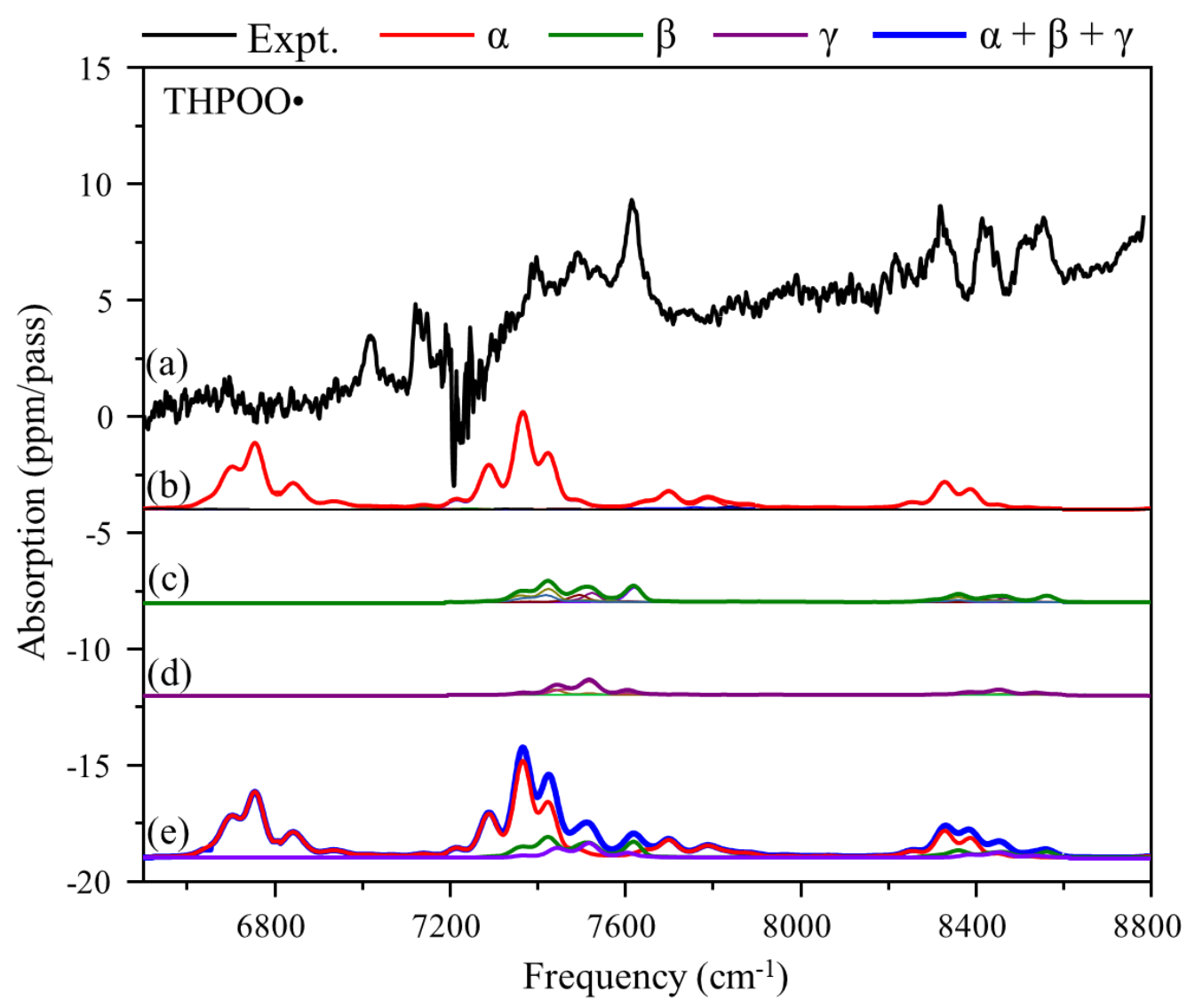

Figure 4.11. (a) CRD spectrum of the THPOO• radical. (b) Simulated spectrum of a-THPOO•. (c) Simulated spectrum of B-THPOO•(d) Simulated spectrum of $\gamma$-THPOO . Thin lines in (b), (c) and (d) are simulated spectra of conformers, while the thick line is their superposition weighted by concentrations. (e) Superposition of the simulated spectra of all regioisomers with a weight of $76.0 \%$ for aTHPOO•, $16.0 \%$ for $6-\mathrm{THPOO} \cdot$, and $8.0 \%$ for $\gamma$-THPOO• Peaks predicted in the region of $6600-7000 \mathrm{~cm}^{-1}$ due to the a-regioisomer are absent in the experiment spectrum. 
Table 4.6. Bond dissociation energy (BDE) of the lowestenergy conformers of all regioisomers of THPOO and THFOO • using the CBS-QB3 method.

\begin{tabular}{cccc} 
Radical & Conformer & Bond & $\begin{array}{c}\mathrm{BDE} \\
(\mathrm{Kcal} / \mathrm{mol})\end{array}$ \\
\hline \multirow{2}{*}{ THPOO $•$} & $\mathrm{a}-\mathrm{Ax}-\mathrm{G}$ & $\mathrm{Ca}^{\prime}-\mathrm{O}$ & 10.3 \\
\cline { 2 - 4 } & $6-\mathrm{Eq}-\mathrm{G}$ & $\mathrm{Ca}-\mathrm{O}$ & 82.8 \\
\cline { 2 - 4 } & $\gamma-\mathrm{Eq}-\mathrm{G}$ & $\mathrm{Ca}-\mathrm{C} 6$ & 84.7 \\
\hline \multirow{2}{*}{ THFOO $•$} & $\mathrm{a}-\mathrm{Ax}-\mathrm{G}^{\prime}$ & $\mathrm{Ca}-\mathrm{O}$ & 82.5 \\
\cline { 2 - 4 } & $6-\mathrm{Eq}-\mathrm{G}^{\prime}$ & $\mathrm{Ca}-\mathrm{O}$ & 79.6 \\
\hline \hline
\end{tabular}

The frequency-scaled spectra of each conformer along with experimental ones are plotted in Figure 4.12 THFOO•, and Figure 4.13 for THPOO•. Simulated spectra of all conformers of each regioisomer are combined to generate spectra of the isomers. Superposition of simulated spectra of regioisomers weighted by their estimated relative populations yields the simulated spectra of the THFOO• and THPOO • radicals. As demonstrated in the figures, the simulation reproduces the experimental spectra well. A spectral simulation of the THPOO• radical with its a-regioisomer included was also attempted. The predicted strong peaks around $6750 \mathrm{~cm}^{-1}$ due to the a-regioisomer is absent in the experimental spectrum, which confirms our assumption that the aregioisomer is not produced in the reaction cell. 


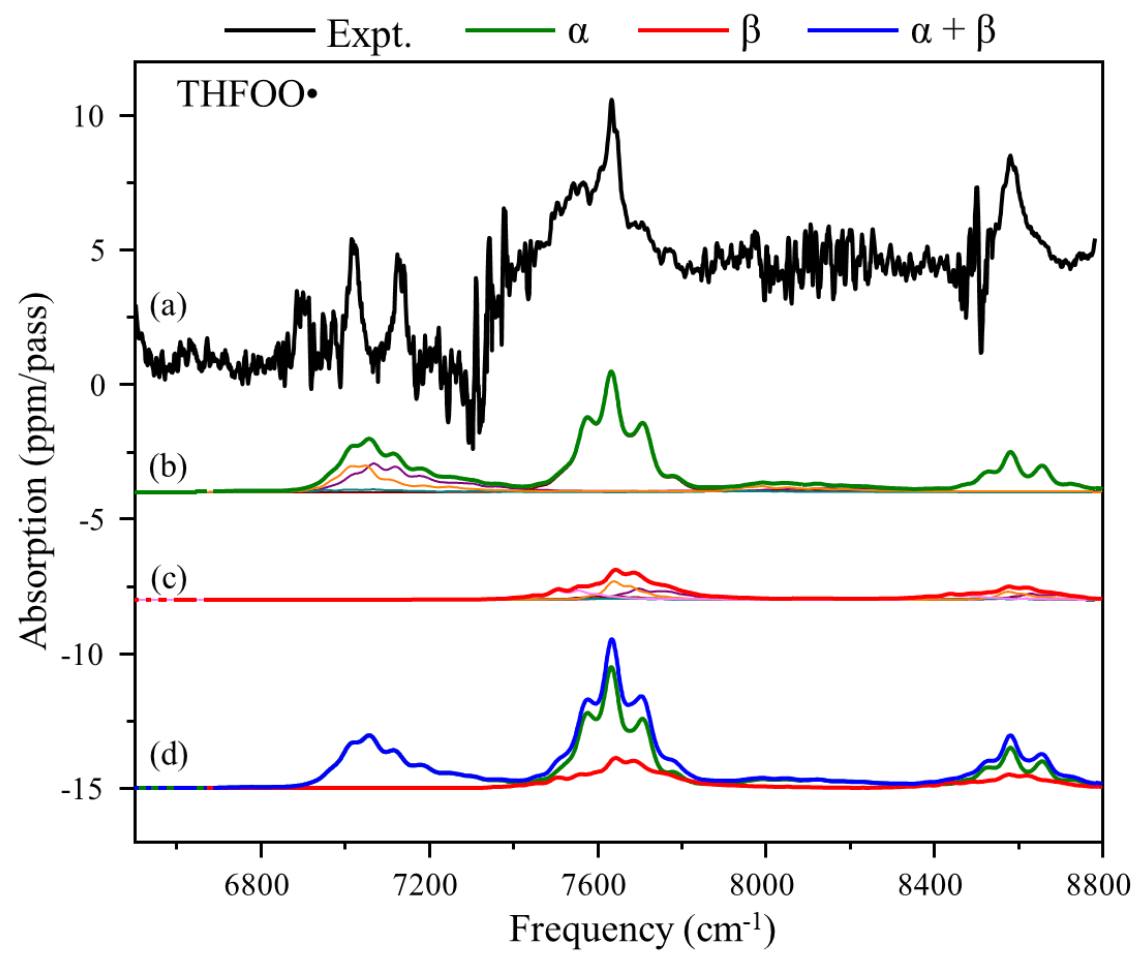

Figure 4.12. (a) CRD spectrum of the THFOO• radical. (b) Simulated spectrum of a-THFOO•. (c) Simulated spectrum of B-THFOO• Thin lines in (b) and (c) are simulated spectra of conformers, while the thick line is their superposition weighted by concentrations. (d) Superposition of the simulated spectra of both regioisomers with a weight of $82.5 \%$ for a-THFOO• and $17.5 \%$ for $B-$ THFOO• 


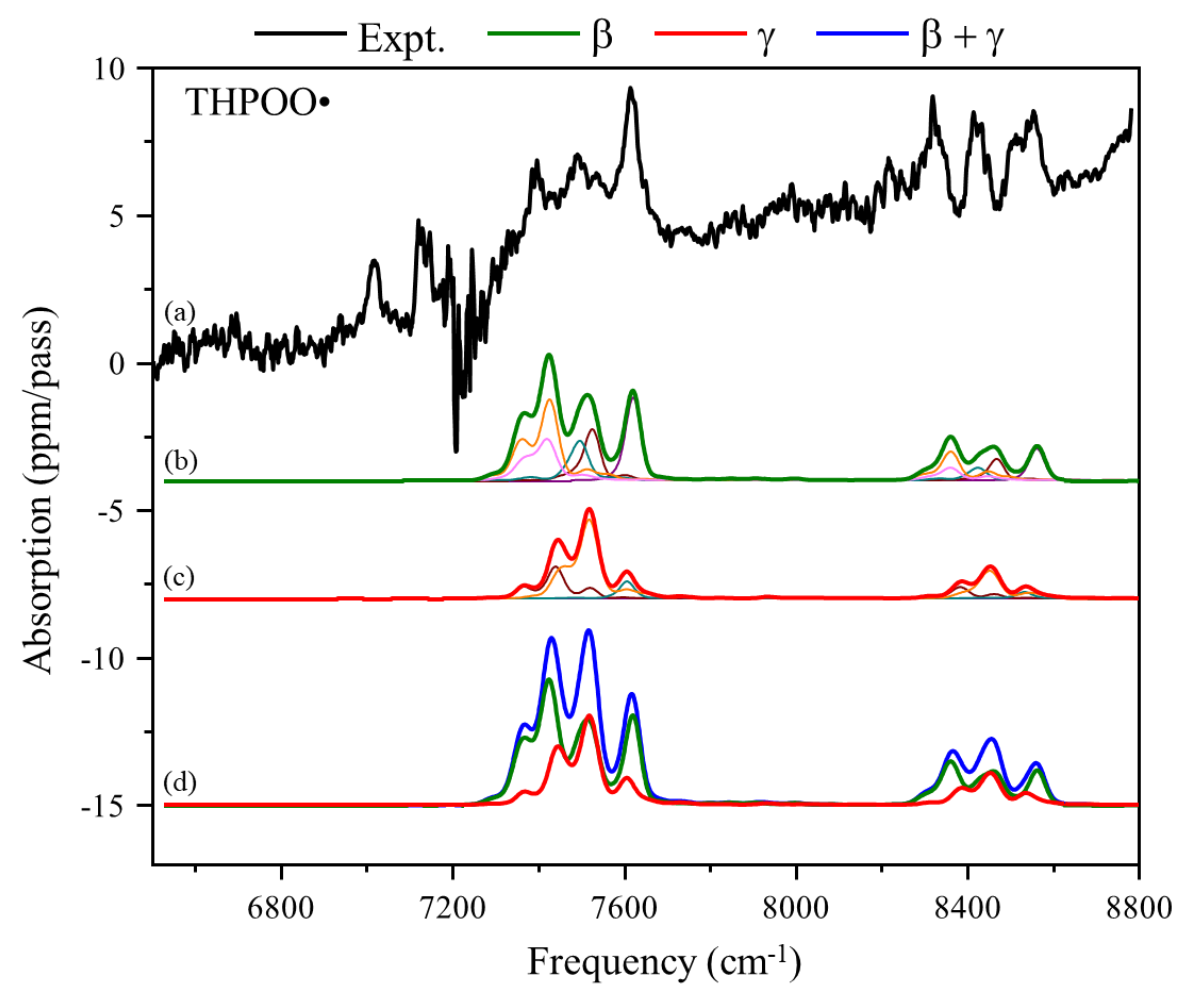

Figure 4.13. (a) CRD spectrum of the THPOO• radical. (b) Simulated spectra of B-THPOO•. (c) Simulated spectra of $\gamma-$ THPOO•. Thin lines in (b) and (c) are simulated spectra of conformers, while the thick line is their superposition weighted by concentrations. (d) Superposition of the simulated spectra of $B-$ and $\gamma$-THPOO - regioisomers with a weight ratio of $2: 1$.

\subsection{Conclusions}

In summary, the THF-yl, and THP-yl peroxy radicals were produced in a reaction cell using $\mathrm{Cl}$-initiated oxidation of THF and THP. The peroxy radicals were detected by CRD spectroscopy of their $\tilde{A} \leftarrow \tilde{X}$ electronic transitions. Quantum chemical calculations predict complex conformational structures of both peroxy radicals. Simulation of transition intensities incorporates the relative populations of the regioisomers and conformers, 
oscillator strengths of the electronic transitions, and FCFs. Frequency-scaled simulation reproduces experimentally obtained spectra of both THFOO• and THPOO•. The strongest peaks in the experimentally observed spectra are assigned to the origin bands and the $\mathrm{OO}$ stretch bands. The simulation also confirms the absence of $\alpha-\mathrm{THPOO} \cdot$ under the current experimental conditions due to ring-opening. Other regioisomers of THFOO and THPOO• have lifetimes between 0.5 and $10 \mathrm{~ms}$ under the current experimental conditions. CRD spectra obtained consist of superimposing rotational contours of multiple regioisomers and conformers. 


\section{CHAPTER 5}

\section{OVERALL CONCLUSIONS}

Measuring dynamics and photophysical properties of materials, molecules, and free radicals are quite essential for understanding their chemical and physical behaviors. Discussed above, two potential renewable energy sources materials and reactive species, perovskite nanomaterials, and peroxy radicals. Overall, the studies presented in this dissertation is in importance for the atmospheric and combustion reactions chemistry, as well as reducing pollution and saving energy.

For implementing new materials as renewable energy sources, kinetics, dynamics photochemical, and physical processes of such material should be understood. Different laser spectroscopy techniques can be employed for this purpose since it is considered a powerful tool for monitoring and probing such processes.

The ultrafast exciton dynamics for methylammonium lead bromide $\left(\mathrm{CH}_{3} \mathrm{NH}_{3} \mathrm{PbBr}_{3}\right)$ nanostructures were studied using transient absorption laser spectroscopy, different charge carrier processes were observed. 
The spectra show four main features that correspond to tow main processes; early-stage dynamics (BGR), evident by a positive PIA signal, and a negative signal due to band-edge SF. Late-stage dynamics (charge recombination) evident by the long-lived SF and a positive intraband absorption signal.

The photoexcitation in the interband continuum is followed by an instantaneous charge carrier thermalization to a quasi-equilibrium distribution, as evidenced by the blue-shifted SF. This SF signal is accompanied by a sub-band-gap PIA signal attributed to photoexcitationinduced transient BGR. The subsequent cooling of hot charge carriers occurs at a sub-picosecond time scale $\left(\tau_{\mathrm{c}} \sim 300 \mathrm{fs}\right)$ and is responsible for the decay of the blue-shifted SF and the PIA signals, as well as the growth of a new longerlasting SF signal, a close-lying SE signal (in the TA spectra of NCs and NPs), and an intraband absorption signal in the blue region. The decay of the SF/SE and the intraband absorption signals follows second-order reaction kinetics, which suggests that the observed charge recombination is dominated by the non-geminate recombination process.

The recombination rate constants demonstrate different dependence on the initial charge carrier density upon photoexcitation. The effective rate constants of the NCs and NPs demonstrate a linear relationship with the initial density of the charge carriers, with the former having a much larger linearity coefficient while that of the NWs remains constant. 
With the same pump fluence and hence similar charge carrier density, both the frequency domain and time domain of NWs are significantly different compared to NCs and NPs due to weaker quantum confinement and less overlap between the electron and hole wave functions. Such size and shape dependences may be utilized for the rational selection of appropriate nanomaterials for the fabrication of high-efficiency solar cells and lightemitting diodes.

THF-yl and THP-yl peroxy radicals were produced in a reaction cell using Cl-initiated oxidation of THF and THP. The peroxy radicals were detected by CRD spectroscopy of their $\tilde{A} \leftarrow \tilde{X}$ electronic transitions. Quantum chemical calculations predict complex conformational structures of both peroxy radicals. Simulation of transition intensities incorporates the relative populations of the regioisomers and conformers, oscillator strengths of the electronic transitions, and FCFs. Frequency-scaled simulation reproduces experimentally obtained spectra of both THFOO • and THPOO • The strongest peaks in the experimentally observed spectra are assigned to the origin bands and the $\mathrm{OO}$ stretch bands. The simulation also confirms the absence of $\mathrm{a}-$ THPOO under the current experimental conditions due to ring-opening. Other regioisomers of THFOO and THPOO have lifetimes between 0.5 and $10 \mathrm{~ms}$ under the current experimental conditions. CRD spectra obtained consist of superimposing rotational contours of multiple regioisomers and conformers. 


\section{CHAPTER 6}

\section{FUTURE STUDIES}

TA spectrum (in the frequency domain) and transient kinetic (in the time domain) of NWs are significantly different compared to NCs and NPs due to weaker quantum confinement and less overlap between the electron and hole wave functions. This suggests a strong effect of quantum confinement on the recombination process.

A series of size or shape-controlled samples can be tested for further information about the effect of quantum confinement on the transient kinetics occurring in perovskite materials. Hence, the selection of appropriate nanomaterials for the fabrication of high-efficiency solar cells and lightemitting diodes.

The complicated spectra obtained by CRDS for those THP-yl and THF-

yl peroxy radicals are due to the spectral congestion as well as the multiple conformers that contribute to the spectra. High-resolution jet cooled CRDS can be utilized using supersonic jet expansion and narrow linewidth lasers to obtain spectral resolution. Also, pre halogenated precursors can be used for obtaining single-isomer spectra. 


\section{REFERENCES}

1. Telfah, H.; Jamhawi, A.; Teunis, M. B.; Sardar, R.; Liu, J., Ultrafast exciton dynamics in shape-controlled methylammonium lead bromide perovskite nanostructures: Effect of quantum confinement on charge carrier recombination. The Journal of Physical Chemistry C 2017, 121 (51), 2855628565.

2. Telfah, H.; Liu, J.; Jamhawi, A.; Strain, J.; Teunis, M.; Sardar, R. In Ultrafast transient absorption spectroscopy investigation of excited state dynamics of methyl ammonium lead bromide perovskite nanostructures, Abstracts of Papers of The American Chemical Society, American Chemical Society 1155 16th St, Nw, Washington, DC 20036 USA: 2017.

3. Telfah, H.; Reza, M. A.; Alam, J.; Paul, A. C.; Liu, J., Direct observation of tetrahydrofuranyl and tetrahydropyranyl peroxy radicals via cavity ringdown spectroscopy. The Journal of Physical Chemistry Letters 2018, 9 (16), 4475-4480.

4. Telfah, H.; Reza, M. A.; Paul, A. C.; Liu, J. In Observation of the $\widetilde{A} \leftarrow \widetilde{X}$ electronic transitions of tetrahydropyranyl and tetrahydrofuranyl peroxy radicals by room-temperature cavity ring-down spectroscopy, 73rd International Symposium on Molecular Spectroscopy, 2018.

5. Panwar, N.; Kaushik, S.; Kothari, S., Role of renewable energy sources in environmental protection: A review. Renewable and Sustainable Energy Reviews 2011, 15 (3), 1513-1524.

6. Liu, M.; Johnston, M. B.; Snaith, H. J., Efficient planar heterojunction perovskite solar cells by vapour deposition. Nature 2013, 501 (7467), 395.

7. Tan, Z.-K.; Moghaddam, R. S.; Lai, M. L.; Docampo, P.; Higler, R.; Deschler, F.; Price, M.; Sadhanala, A.; Pazos, L. M.; Credgington, D., Bright light-emitting diodes based on organometal halide perovskite. Nature Nanotechnology 2014, 9 (9), 687.

8. $\quad$ Fang, Y.; Dong, Q.; Shao, Y.; Yuan, Y.; Huang, J., Highly narrowband perovskite single-crystal photodetectors enabled by surface-charge recombination. Nature Photonics 2015, $9(10), 679$.

9. Z Zhu, H.; Fu, Y.; Meng, F.; Wu, X.; Gong, Z.; Ding, Q.; Gustafsson, M. V.; Trinh, M. T.; Jin, S.; Zhu, X., Lead halide perovskite nanowire lasers with low lasing thresholds and high quality factors. Nature Materials 2015, 14 (6), 636. 10. Miyata, A.; Mitioglu, A.; Plochocka, P.; Portugall, O.; Wang, J. T.-W.; Stranks, S. D.; Snaith, H. J.; Nicholas, R. J., Direct measurement of the exciton binding energy and effective masses for charge carriers in organic-inorganic tri-halide perovskites. Nature Physics 2015, 11 (7), 582. 
11. Stranks, S. D.; Eperon, G. E.; Grancini, G.; Menelaou, C.; Alcocer, M. J.; Leijtens, T.; Herz, L. M.; Petrozza, A.; Snaith, H. J., Electron-hole diffusion lengths exceeding 1 micrometer in an organometal trihalide perovskite absorber. Science 2013, 342 (6156), 341-344.

12. Kumawat, N. K.; Dey, A.; Kumar, A.; Gopinathan, S. P.; Narasimhan, K.; Kabra, D., Band Gap Tuning of $\mathrm{CH}_{3} \mathrm{NH}_{3} \mathrm{~Pb}\left(\mathrm{Br}_{1-\mathrm{x}} \mathrm{Cl}_{\mathrm{x}}\right)_{3}$ Hybrid Perovskite for Blue Electroluminescence. ACS Applied Materials \& Interfaces 2015, 7 (24), 13119-13124.

13. Kojima, A.; Teshima, K.; Shirai, Y.; Miyasaka, T., Organometal halide perovskites as visible-light sensitizers for photovoltaic cells. Journal of the American Chemical Society 2009, 131 (17), 6050-6051.

14. Shin, S. S.; Yeom, E. J.; Yang, W. S.; Hur, S.; Kim, M. G.; Im, J.; Seo, J.; Noh, J. H.; Seok, S. I., Colloidally prepared La-doped $\mathrm{BaSnO}_{3}$ electrodes for efficient, photostable perovskite solar cells. Science 2017, 356 (6334), 167-171. 15. Castelli, I. E.; Pandey, M.; Thygesen, K. S.; Jacobsen, K. W., Band-gap engineering of functional perovskites through quantum confinement and tunneling. Physical Review B 2015, 91 (16), 165309.

16. Wu, X.; Trinh, M. T.; Niesner, D.; Zhu, H.; Norman, Z.; Owen, J. S.; Yaffe, O.; Kudisch, B. J.; Zhu, X.-Y., Trap states in lead iodide perovskites. Journal of the American Chemical Society 2015, 137 (5), 2089-2096.

17. Zhang, W.; Anaya, M.; Lozano, G.; Calvo, M. E.; Johnston, M. B.; Míguez, H. n.; Snaith, H. J., Highly efficient perovskite solar cells with tunable structural color. Nano Letters 2015, 15 (3), 1698-1702.

18. Yin, W.-J.; Shi, T.; Yan, Y., Unusual defect physics in $\mathrm{CH}_{3} \mathrm{NH}_{3} \mathrm{PbI}_{3}$ perovskite solar cell absorber. Applied Physics Letters 2014, 104 (6), 063903.

19. Yang, Y.; Yang, M.; Li, Z.; Crisp, R.; Zhu, K.; Beard, M. C., Comparison of recombination dynamics in $\mathrm{CH}_{3} \mathrm{NH}_{3} \mathrm{PbBr}_{3}$ and $\mathrm{CH}_{3} \mathrm{NH}_{3} \mathrm{PbI}_{3}$ perovskite films: influence of exciton binding energy. The Journal of Physical Chemistry Letters 2015, 6 (23), 4688-4692.

20. Rotavera, B.; Savee, J. D.; Antonov, I. O.; Caravan, R. L.; Sheps, L.; Osborn, D. L.; Zádor, J.; Taatjes, C. A., Influence of oxygenation in cyclic hydrocarbons on chain-termination reactions from $\mathrm{R}+\mathrm{O}_{2}$ : tetrahydropyran and cyclohexane. Proceedings of the Combustion Institute 2017, 36 (1), 597-606.

21. West, R. M.; Kunkes, E. L.; Simonetti, D. A.; Dumesic, J. A., Catalytic conversion of biomass-derived carbohydrates to fuels and chemicals by formation and upgrading of mono-functional hydrocarbon intermediates. Catalysis Today 2009, 147 (2), 115-125.

22. Rienstra-Kiracofe, J. C.; Allen, W. D.; Schaefer, H. F., The $\mathrm{C}_{2} \mathrm{H}_{5}+\mathrm{O}_{2}$ reaction mechanism: high-level ab initio characterizations. The Journal of Physical Chemistry A 2000, 104 (44), 9823-9840.

23. Pilling, M. J., Interactions between theory and experiment in the investigation of elementary reactions of importance in combustion. Chemical Society Reviews 2008, 37 (4), 676-685. 
24. Criegee, R., Mechanism of ozonolysis. Angewandte Chemie International Edition in English 1975, 14 (11), 745-752.

25. Beames, J. M.; Liu, F.; Lu, L.; Lester, M. I., Ultraviolet spectrum and photochemistry of the simplest Criegee intermediate $\mathrm{CH}_{2} \mathrm{OO}$. Journal of the American Chemical Society 2012, 134 (49), 20045-20048.

26. Taatjes, C. A.; Meloni, G.; Selby, T. M.; Trevitt, A. J.; Osborn, D. L.; Percival, C. J.; Shallcross, D. E., Direct observation of the gas-phase Criegee intermediate $\left(\mathrm{CH}_{2} \mathrm{OO}\right)$. Journal of the American Chemical Society 2008, 130 (36), 11883-11885.

27. Su, Y.-T.; Huang, Y.-H.; Witek, H. A.; Lee, Y.-P., Infrared absorption spectrum of the simplest Criegee intermediate $\mathrm{CH}_{2} \mathrm{OO}$. Science 2013, 340 (6129), 174-176.

28. Johnson, D.; Marston, G., The gas-phase ozonolysis of unsaturated volatile organic compounds in the troposphere. Chemical Society Reviews 2008, $37(4), 699-716$.

29. Kim, H.-S.; Lee, C.-R.; Im, J.-H.; Lee, K.-B.; Moehl, T.; Marchioro, A.; Moon, S.-J.; Humphry-Baker, R.; Yum, J.-H.; Moser, J. E., Lead iodide perovskite sensitized all-solid-state submicron thin film mesoscopic solar cell with efficiency exceeding 9\%. Scientific Reports 2012, 2, 591.

30. Burschka, J.; Pellet, N.; Moon, S.-J.; Humphry-Baker, R.; Gao, P.; Nazeeruddin, M. K.; Grätzel, M., Sequential deposition as a route to highperformance perovskite-sensitized solar cells. Nature 2013, 499 (7458), 316.

31. Noel, N. K.; Abate, A.; Stranks, S. D.; Parrott, E. S.; Burlakov, V. M.; Goriely, A.; Snaith, H. J., Enhanced photoluminescence and solar cell performance via Lewis base passivation of organic-inorganic lead halide perovskites. ACS Nano 2014, 8(10), 9815-9821.

32. Zhou, H.; Chen, Q.; Li, G.; Luo, S.; Song, T.-b.; Duan, H.-S.; Hong, Z.; You, J.; Liu, Y.; Yang, Y., Interface engineering of highly efficient perovskite solar cells. Science 2014, 345 (6196), 542-546.

33. Park, N.-G., Perovskite solar cells: an emerging photovoltaic technology. Materials Today 2015, 18 (2), 65-72.

34. Chart, N. E., NREL Efficiency Chart.(2018). ed.

35. Im, J.-H.; Lee, C.-R.; Lee, J.-W.; Park, S.-W.; Park, N.-G., 6.5\% efficient perovskite quantum-dot-sensitized solar cell. Nanoscale 2011, 3 (10), 40884093.

36. Lee, M. M.; Teuscher, J.; Miyasaka, T.; Murakami, T. N.; Snaith, H. J., Efficient hybrid solar cells based on meso-superstructured organometal halide perovskites. Science 2012, 338 (6107), 643-647.

37. Liu, D.; Kelly, T. L., Perovskite solar cells with a planar heterojunction structure prepared using room-temperature solution processing techniques. Nature Photonics 2014, 8(2), 133.

38. Xing, G.; Mathews, N.; Lim, S. S.; Yantara, N.; Liu, X.; Sabba, D.; Grätzel, M.; Mhaisalkar, S.; Sum, T. C., Low-temperature solution-processed wavelength-tunable perovskites for lasing. Nature Materials 2014, 13 (5), 476. 
39. Piatkowski, P.; Cohen, B.; Ramos, F. J.; Di Nunzio, M.; Nazeeruddin, M. K.; Grätzel, M.; Ahmad, S.; Douhal, A., Direct monitoring of ultrafast electron and hole dynamics in perovskite solar cells. Physical Chemistry Chemical Physics 2015, 17 (22), 14674-14684.

40. Manser, J. S.; Kamat, P. V., Band filling with free charge carriers in organometal halide perovskites. Nature Photonics 2014, 8(9), 737.

41. Xing, G.; Mathews, N.; Sun, S.; Lim, S. S.; Lam, Y. M.; Grätzel, M.; Mhaisalkar, S.; Sum, T. C., Long-range balanced electron-and hole-transport lengths in organic-inorganic $\mathrm{CH}_{3} \mathrm{NH}_{3} \mathrm{PbI}_{3}$. Science 2013, 342 (6156), 344-347.

42. Marchioro, A.; Brauer, J. C.; Teuscher, J.; Grätzel, M.; Moser, J.-E. In Photoinduced processes in lead iodide perovskite solid-state solar cells, Physical Chemistry of Interfaces and Nanomaterials XII, International Society for Optics and Photonics: 2013; p 881108.

43. Ahmed, G. H.; Liu, J.; Parida, M. R.; Murali, B.; Bose, R.; AlYami, N. M.; Hedhili, M. N.; Peng, W.; Pan, J.; Besong, T. M., Shape-tunable charge carrier dynamics at the interfaces between perovskite nanocrystals and molecular acceptors. The Journal of Physical Chemistry Letters 2016, 7 (19), 3913-3919.

44. Wu, X.; Trinh, M. T.; Zhu, X.-Y., Excitonic many-body interactions in two-dimensional lead iodide perovskite quantum wells. The Journal of Physical Chemistry C 2015, 119 (26), 14714-14721.

45. Lindblad, R.; Bi, D.; Park, B.-'w.; Oscarsson, J.; Gorgoi, M.; Siegbahn, H.; Odelius, M.; Johansson, E. M.; Rensmo, H. k., Electronic structure of $\mathrm{TiO}_{2} / \mathrm{CH}_{3} \mathrm{NH}_{3} \mathrm{PbI}_{3}$ perovskite solar cell interfaces. The Journal of Physical Chemistry Letters 2014, 5 (4), 648-653.

46. Zhu, Z.; Ma, J.; Wang, Z.; Mu, C.; Fan, Z.; Du, L.; Bai, Y.; Fan, L.; Yan, H.; Phillips, D. L., Efficiency enhancement of perovskite solar cells through fast electron extraction: the role of graphene quantum dots. Journal of the American Chemical Society 2014, 136 (10), 3760-3763.

47. De Bastiani, M.; D’Innocenzo, V.; Stranks, S. D.; Snaith, H. J.; Petrozza, A., Role of the crystallization substrate on the photoluminescence properties of organo-lead mixed halides perovskites. APL Materials 2014, 2 (8), 081509.

48. Grancini, G.; Marras, S.; Prato, M.; Giannini, C.; Quarti, C.; De Angelis, F.; De Bastiani, M.; Eperon, G. E.; Snaith, H. J.; Manna, L., The impact of the crystallization processes on the structural and optical properties of hybrid perovskite films for photovoltaics. The Journal of Physical Chemistry Letters 2014, 5 (21), 3836-3842.

49. Teunis, M. B.; Jana, A.; Dutta, P.; Johnson, M. A.; Mandal, M.; Muhoberac, B. B.; Sardar, R., Mesoscale growth and assembly of bright luminescent organolead halide perovskite quantum wires. Chemistry of Materials 2016, 28 (14), 5043-5054.

50. Lightfoot, P. D.; Cox, R.; Crowley, J.; Destriau, M.; Hayman, G.; Jenkin, M.; Moortgat, G.; Zabel, F., Organic peroxy radicals: kinetics, spectroscopy and 
tropospheric chemistry. Atmospheric Environment. Part A. General Topics 1992, 26 (10), 1805-1961.

51. Pilling, M. J., Low-temperature combustion and autoignition. Elsevier: 1997; Vol. 35.

52. Zádor, J.; Taatjes, C. A.; Fernandes, R. X., Kinetics of elementary reactions in low-temperature autoignition chemistry. Progress in Energy and Combustion Science 2011, 37(4), 371-421.

53. Osborn, D. L., Reaction mechanisms on multiwell potential energy surfaces in combustion (and atmospheric) chemistry. Annual Review of Physical Chemistry 2017, 68, 233-260.

54. Orlando, J. J.; Tyndall, G. S., Laboratory studies of organic peroxy radical chemistry: an overview with emphasis on recent issues of atmospheric significance. Chemical Society Reviews 2012, 41 (19), 6294-6317.

55. Orlando, J. J.; Tyndall, G. S.; Wallington, T. J., The atmospheric chemistry of alkoxy radicals. Chemical reviews 2003, 103 (12), 4657-4690.

56. Carstensen, H.-H.; Naik, C. V.; Dean, A. M., Detailed Modeling of the Reaction of $\mathrm{C}_{2} \mathrm{H}_{5}+\mathrm{O}_{2}$. The Journal of Physical Chemistry A 2005, 109 (10), 2264-2281.

57. Curran, H. J.; Gaffuri, P.; Pitz, W. J.; Westbrook, C. K., A comprehensive modeling study of n-heptane oxidation. Combustion and Flame 1998, 114 (12), 149-177.

58. Wang, S.; Miller, D. L.; Cernansky, N. P.; Curran, H. J.; Pitz, W. J.; Westbrook, C. K., A flow reactor study of neopentane oxidation at 8 atmospheres: experiments and modeling. Combustion and Flame 1999, 118 (3), 415-430.

59. Knox, J. H., The interpretation of cool flame and low-temperature combustion phenomena. Photochemistry and Reaction Kinetics (ed. PG Ashmore, FS Dainton \& TM Sugden) 1967, 250-286.

60. Glassman, I., Combustion, Academic Press Orlando FL. 1987.

61. Gaffuri, P.; Faravelli, T.; Ranzi, E.; Cernansky, N. P.; Miller, D.; d'Anna, A.; Ciajolo, A., Comprehensive kinetic model for the low-temperature oxidation of hydrocarbons. AIChE Journal 1997, 43 (5), 1278-1286.

62. Just, G. M.; Sharp, E. N.; Zalyubovsky, S. J.; Miller, T. A., Cavity ringdown spectroscopy of the $\widetilde{\mathrm{A}} \leftarrow \widetilde{\mathrm{X}}$ electronic transition of the phenyl peroxy radical. Chemical Physics Letters 2006, 417(4-6), 378-382.

63. Morley, C., A fundamentally based correlation between alkane structure and octane number. Combustion Science and Technology 1987, 55 (4-6), 115123.

64. Warnatz, J.; Maas, U.; Dibble, R. W., Physical and chemical fundamentals, modeling and simulation, experiments, pollutant formation. Springer, Berlin Frenklach M, Wang H (1990) Detailed kinetic modelling of soot particle nucleation and growth. Proceedings of the Combustion Institute 2006, 23, 1559-1566. 
65. Westbrook, C., Chemistry behind engine knock. Chemistry and Industry 1992, 562-6.

66. Guenther, A.; Hewitt, C. N.; Erickson, D.; Fall, R.; Geron, C.; Graedel, T.; Harley, P.; Klinger, L.; Lerdau, M.; McKay, W., A global model of natural volatile organic compound emissions. Journal of Geophysical Research: Atmospheres 1995, 100 (D5), 8873-8892.

67. Sobanski, N.; Schuladen, J.; Schuster, G.; Lelieveld, J.; Crowley, J. N., A five-channel cavity ring-down spectrometer for the detection of $\mathrm{NO}_{2}, \mathrm{NO}_{3}$, $\mathrm{N}_{2} \mathrm{O}_{5}$, total peroxy nitrates and total alkyl nitrates. Atmospheric Measurement Techniques 2016, 9, 5103-5118.

68. Roehl, C. M.; Orlando, J. J.; Tyndall, G. S.; Shetter, R. E.; Vazquez, G. J.; Cantrell, C. A.; Calvert, J. G., Temperature dependence of the quantum yields for the photolysis of $\mathrm{NO}_{2}$ near the dissociation limit. The Journal of Physical Chemistry 1994, 98 (32), 7837-7843.

69. Besler, B. H.; Sevilla, M. D.; MacNeille, P., Ab initio studies of hydrocarbon peroxyl radicals. The Journal of Physical Chemistry 1986, 90 (24), 6446-6451.

70. Bair, R. A.; Goddard III, W. A., Ab initio studies of the structures of peroxides and peroxy radicals. Journal of the American Chemical Society 1982, 104 (10), 2719-2724.

71. Aplincourt, P.; Ruiz-López, M. F.; Assfeld, X.; Bohr, F., Structure of isolated and solvated peroxyl radicals. Journal of Computational Chemistry 1999, 20 (10), 1039-1048.

72. Boyd, S. L.; Boyd, R. J.; Barclay, L. R. C., A theoretical investigation of the structures and properties of peroxyl radicals. Journal of the American Chemical Society 1990, 112 (15), 5724-5730.

73. Brinck, T.; Lee, H.-N.; Jonsson, M., Quantum chemical studies on the thermochemistry of alkyl and peroxyl radicals. The Journal of Physical Chemistry A 1999, 103 (35), 7094-7104.

74. Jafri, J. A.; Phillips, D. H., Ground and lower excited states of methylperoxy radical, $\mathrm{CH}_{3} \mathrm{O}_{2}$ : a computational investigation. Journal of the American Chemical Society 1990, 112 (7), 2586-2590.

75. Stark, M. S., Addition of peroxyl radicals to alkenes and the reaction of oxygen with alkyl radicals. Journal of the American Chemical Society 2000, $122(17), 4162-4170$.

76. Weisman, J. L.; Head-Gordon, M., Origin of substituent effects in the absorption spectra of peroxy radicals: Time dependent density functional theory calculations. Journal of the American Chemical Society 2001, 123 (47), 11686-11694.

77. Murrells, T. P.; Jenkin, M. E.; Shalliker, S. J.; Hayman, G. D., Lasser flash photolysis study of the UV spectrum and kinetics of reactions of $\mathrm{HOCH}_{2} \mathrm{CH}_{2} \mathrm{O}_{2}$ radicals. Journal of the Chemical Society, Faraday Transactions 1991, 87(15), 2351-2360. 
78. Wallington, T.; Dagaut, P.; Kurylo, M., UV absorption cross sections and reaction kinetics and mechanisms for peroxy radicals in the gas phase. Chemical Reviews 1992, 92 (4), 667-710.

79. Villenave, E.; Lesclaux, R., Kinetics of the cross reactions of $\mathrm{CH}_{3} \mathrm{O}_{2}$ and $\mathrm{C}_{2} \mathrm{H}_{5} \mathrm{O}_{2}$ radicals with selected peroxy radicals. The Journal of Physical Chemistry 1996, 100 (34), 14372-14382.

80. Newman, S. M.; Lane, I. C.; Orr-Ewing, A. J.; Newnham, D. A.; Ballard, J., Integrated absorption intensity and Einstein coefficients for the $\mathrm{O}_{2}$ a ${ }^{1} \Delta_{\mathrm{g}}-\mathrm{X}$ ${ }^{3} \Sigma_{\mathrm{g}}-(0,0)$ transition: A comparison of cavity ringdown and high resolution Fourier transform spectroscopy with a long-path absorption cell. The Journal of Chemical Physics 1999, 110 (22), 10749-10757.

81. Alfano, R. R., The supercontinuum laser source. 1989.

82. Berden, G.; Peeters, R.; Meijer, G., Cavity ring-down spectroscopy: Experimental schemes and applications. International Reviews in Physical Chemistry 2000, 19 (4), 565-607.

83. O'Keefe, A.; Deacon, D. A., Cavity ring-down optical spectrometer for absorption measurements using pulsed laser sources. Review of Scientific Instruments 1988, 59 (12), 2544-2551.

84. Vallance, C., Innovations in cavity ringdown spectroscopy. New Journal of Chemistry 2005, 29 (7), 867-874.

85. Brown, S. S., Absorption spectroscopy in high-finesse cavities for atmospheric studies. Chemical Reviews 2003, 103 (12), 5219-5238.

86. Eskola, A.; Carr, S.; Blitz, M.; Pilling, M.; Seakins, P., Kinetics and yields of $\mathrm{OH}$ radical from the $\mathrm{CH}_{3} \mathrm{OCH}_{2}+\mathrm{O}_{2}$ reaction using a new photolytic source. Chemical Physics Letters 2010, $487(1-3), 45-50$.

87. Eskola, A.; Carr, S.; Shannon, R.; Wang, B.; Blitz, M.; Pilling, M.; Seakins, P.; Robertson, S., Analysis of the Kinetics and Yields of $\mathrm{OH}$ Radical Production from the $\mathrm{CH}_{3} \mathrm{OCH}_{2}+\mathrm{O}_{2}$ Reaction in the Temperature Range 195$650 \mathrm{~K}$ : an Experimental and Computational study. The Journal of Physical Chemistry A 2014, 118 (34), 6773-6788.

88. Michaela, J.; Nava, D.; Payne, W.; Stiefb, L., Rate constants for the reaction of atomic chlorine with methanol and dimethyl ether from 200 to 500 K. The Journal of Chemical Physics 1979, 70 (8), 3652-3656.

89. Owyoung, A., High-resolution cw stimulated Raman spectroscopy in molecular hydrogen. Optics Letters 1978, 2 (4), 91-93.

90. Submann, R.; Weber, T.; Riedle, E.; Neusser, H., Frequency shifting of pulsed narrow-band laser light in a multipass Raman cell. Optics Communications 1992, 88 (4-6), 408-414.

91. Reza, M. A.; Telfah, H.; Xu, R.; Liu, J., Room-temperature cavity ringdown spectroscopy of methylallyl peroxy radicals. The Journal of Physical Chemistry A 2019, 123 (16), 3510-3517.

92. Grancini, G.; Kandada, A. R. S.; Frost, J. M.; Barker, A. J.; De Bastiani, M.; Gandini, M.; Marras, S.; Lanzani, G.; Walsh, A.; Petrozza, A., Role of 
microstructure in the electron-hole interaction of hybrid lead halide perovskites. Nature Photonics 2015, 9 (10), 695.

93. de Quilettes, D. W.; Vorpahl, S. M.; Stranks, S. D.; Nagaoka, H.; Eperon, G. E.; Ziffer, M. E.; Snaith, H. J.; Ginger, D. S., Impact of microstructure on local carrier lifetime in perovskite solar cells. Science 2015, 348 (6235), 683686.

94. Tyagi, P.; Arveson, S. M.; Tisdale, W. A., Colloidal organohalide perovskite nanoplatelets exhibiting quantum confinement. The Journal of Physical Chemistry Letters 2015, 6 (10), 1911-1916.

95. Luo, B.; Pu, Y.-C.; Yang, Y.; Lindley, S. A.; Abdelmageed, G.; Ashry, H.; Li, Y.; Li, X.; Zhang, J. Z., Synthesis, optical properties, and exciton dynamics of organolead bromide perovskite nanocrystals. The Journal of Physical Chemistry C 2015, 119 (47), 26672-26682.

96. Sichert, J. A.; Tong, Y.; Mutz, N.; Vollmer, M.; Fischer, S.; Milowska, K. Z.; García Cortadella, R.; Nickel, B.; Cardenas-Daw, C.; Stolarczyk, J. K., Quantum size effect in organometal halide perovskite nanoplatelets. Nano Letters 2015, 15 (10), 6521-6527.

97. Zheng, K.; Abdellah, M.; Zhu, Q.; Kong, Q.; Jennings, G.; Kurtz, C. A.; Messing, M. E.; Niu, Y.; Gosztola, D. J.; Al-Marri, M. J., Direct experimental evidence for photoinduced strong-coupling polarons in organolead halide perovskite nanoparticles. The Journal of Physical Chemistry Letters 2016, 7 (22), 4535-4539.

98. Chung, H.; Jung, S. I.; Kim, H. J.; Cha, W.; Sim, E.; Kim, D.; Koh, W. K.; Kim, J., Composition-dependent hot carrier relaxation dynamics in cesium lead halide $\left(\mathrm{CsPbX}_{3}, \mathrm{X}=\mathrm{Br}\right.$ and I) perovskite nanocrystals. Angewandte Chemie International Edition 2017, 56 (15), 4160-4164.

99. Bouduban, M. E.; Burgos-Caminal, A.; Ossola, R.; Teuscher, J.; Moser, J.-E., Energy and charge transfer cascade in methylammonium lead bromide perovskite nanoparticle aggregates. Chemical Science 2017, 8 (6), 4371-4380. 100. Papagiorgis, P.; Protesescu, L.; Kovalenko, M. V.; Othonos, A.; Itskos, G., Long-lived hot carriers in formamidinium lead iodide nanocrystals. The Journal of Physical Chemistry C 2017, 121 (22), 12434-12440.

101. Wang, L.; Williams, N. E.; Malachosky, E. W.; Otto, J. P.; Hayes, D.; Wood, R. E.; Guyot-Sionnest, P.; Engel, G. S., Scalable ligand-mediated transport synthesis of organic-inorganic hybrid perovskite nanocrystals with resolved electronic structure and ultrafast dynamics. ACS Nano 2017, 11 (3), 2689-2696.

102. Butkus, J.; Vashishtha, P.; Chen, K.; Gallaher, J. K.; Prasad, S. K.; Metin, D. Z.; Laufersky, G.; Gaston, N.; Halpert, J. E.; Hodgkiss, J. M., The evolution of quantum confinement in $\mathrm{CsPbBr}_{3}$ perovskite nanocrystals. Chemistry of Materials 2017, 29 (8), 3644-3652.

103. Harrison, P.; Valavanis, A., Quantum wells, wires and dots: theoretical and computational physics of semiconductor nanostructures. John Wiley \& Sons: 2016. 
104. Koutselas, I.; Ducasse, L.; Papavassiliou, G. C., Electronic properties of three-and low-dimensional semiconducting materials with $\mathrm{Pb}$ halide and $\mathrm{Sn}$ halide units. Journal of Physics: Condensed Matter 1996, 8 (9), 1217.

105. Einevoll, G., Confinement of excitons in quantum dots. Physical Review $B 1992,45(7), 3410$.

106. Teunis, M. B.; Lawrence, K. N.; Dutta, P.; Siegel, A. P.; Sardar, R., Pure white-light emitting ultrasmall organic-inorganic hybrid perovskite nanoclusters. Nanoscale 2016, 8 (40), 17433-17439.

107. Pandit, B.; Luitel, T.; Cummins, D. R.; Thapa, A. K.; Druffel, T.; Zamborini, F.; Liu, J., Spectroscopic investigation of photoinduced chargetransfer processes in $\mathrm{FTO} / \mathrm{TiO}_{2} / \mathrm{N} 719$ photoanodes with and without covalent attachment through silane-based linkers. The Journal of Physical Chemistry A 2013, 117 (50), 13513-13523.

108. Xie, Y.; Teunis, M. B.; Pandit, B.; Sardar, R.; Liu, J., Molecule-like CdSe nanoclusters passivated with strongly interacting ligands: energy level alignment and photoinduced ultrafast charge transfer processes. The Journal of Physical Chemistry C 2015, 119 (5), 2813-2821.

109. Saidaminov, M. I.; Abdelhady, A. L.; Murali, B.; Alarousu, E.; Burlakov, V. M.; Peng, W.; Dursun, I.; Wang, L.; He, Y.; Maculan, G., High-quality bulk hybrid perovskite single crystals within minutes by inverse temperature crystallization. Nature Communications 2015, 6, 7586.

110. Shi, D.; Adinolfi, V.; Comin, R.; Yuan, M.; Alarousu, E.; Buin, A.; Chen, Y.; Hoogland, S.; Rothenberger, A.; Katsiev, K., Low trap-state density and long carrier diffusion in organolead trihalide perovskite single crystals. Science 2015, 347 (6221), 519-522.

111. Ithurria, S.; Tessier, M.; Mahler, B.; Lobo, R.; Dubertret, B.; Efros, A. L., Colloidal nanoplatelets with two-dimensional electronic structure. Nature Materials 2011, 10 (12), 936.

112. Wang, Y.; Herron, N., Nanometer-sized semiconductor clusters: materials synthesis, quantum size effects, and photophysical properties. The Journal of Physical Chemistry 1991, 95 (2), 525-532.

113. Urbach, F., The long-wavelength edge of photographic sensitivity and of the electronic absorption of solids. Physical Review 1953, 92 (5), 1324.

114. Singh, S.; Li, C.; Panzer, F.; Narasimhan, K.; Graeser, A.; Gujar, T. P.; Kohler, A.; Thelakkat, M.; Huettner, S.; Kabra, D., Effect of thermal and structural disorder on the electronic structure of hybrid perovskite semiconductor $\mathrm{CH}_{3} \mathrm{NH}_{3} \mathrm{PbI}_{3}$. The Journal of Physical Chemistry Letters 2016, 7(15), 3014-3021.

115. Xu, J.; Zhuang, X.; Guo, P.; Zhang, Q.; Huang, W.; Wan, Q.; Hu, W.; Wang, X.; Zhu, X.; Fan, C., Wavelength-converted/selective waveguiding based on composition-graded semiconductor nanowires. Nano Letters 2012, 12 (9), 5003-5007.

116. Mahan, G., Phonon-broadened optical spectra: Urbach's rule. Physical Review 1966, 145 (2), 602. 
117. Liu, Y.-H.; Wang, F.; Wang, Y.; Gibbons, P. C.; Buhro, W. E., Lamellar assembly of cadmium selenide nanoclusters into quantum belts. Journal of the American Chemical Society 2011, 133 (42), 17005-17013.

118. Sun, S.; Yuan, D.; Xu, Y.; Wang, A.; Deng, Z., Ligand-mediated synthesis of shape-controlled cesium lead halide perovskite nanocrystals via reprecipitation process at room temperature. ACS Nano 2016, 10 (3), 36483657.

119. Chamarro, M.; Gourdon, C.; Lavallard, P.; Lublinskaya, O.; Ekimov, A., Enhancement of electron-hole exchange interaction in CdSe nanocrystals: A quantum confinement effect. Physical Review B 1996, 53 (3), 1336.

120. Efros, A. L.; Rosen, M.; Kuno, M.; Nirmal, M.; Norris, D. J.; Bawendi, M., Band-edge exciton in quantum dots of semiconductors with a degenerate valence band: Dark and bright exciton states. Physical Review B 1996, 54 (7), 4843.

121. Chamarro, M.; Dib, M.; Voliotis, V.; Filoramo, A.; Roussignol, P.; Gacoin, T.; Boilot, J.; Delerue, C.; Allan, G.; Lannoo, M., Interplay of coulomb, exchange, and spin-orbit effects in semiconductor nanocrystallites. Physical Review B 1998, 57 (7), 3729.

122. Perez-Conde, J.; Bhattacharjee, A.; Chamarro, M.; Lavallard, P.; Petrikov, V.; Lipovskii, A., Photoluminescence Stokes shift and exciton fine structure in CdTe nanocrystals. Physical Review B 2001, 64 (11), 113303.

123. Couteau, C.; Larrue, A.; Wilhelm, C.; Soci, C., Nanowire lasers. Nanophotonics 2015, 4 (1), 90-107.

124. Ma, Y.; Guo, X.; Wu, X.; Dai, L.; Tong, L., Semiconductor nanowire lasers. Advances in Optics and Photonics 2013, 5 (3), 216-273.

125. Shah, J., Ultrafast spectroscopy of semiconductors and semiconductor nanostructures. Springer Science \& Business Media: 2013; Vol. 115.

126. Yang, Y.; Ostrowski, D. P.; France, R. M.; Zhu, K.; Van De Lagemaat, J.; Luther, J. M.; Beard, M. C., Observation of a hot-phonon bottleneck in leadiodide perovskites. Nature Photonics 2016, 10 (1), 53.

127. Shah, J.; Leite, R., Radiative recombination from photoexcited hot carriers in GaAs. Physical Review Letters 1969, 22 (24), 1304.

128. Shank, C.; Fork, R.; Leheny, R.; Shah, J., Dynamics of photoexcited GaAs band-edge absorption with subpicosecond resolution. Physical Review Letters 1979, 42 (2), 112.

129. Price, M. B.; Butkus, J.; Jellicoe, T. C.; Sadhanala, A.; Briane, A.; Halpert, J. E.; Broch, K.; Hodgkiss, J. M.; Friend, R. H.; Deschler, F., Hotcarrier cooling and photoinduced refractive index changes in organic-inorganic lead halide perovskites. Nature Communications 2015, 6, 8420.

130. Deschler, F.; Price, M.; Pathak, S.; Klintberg, L. E.; Jarausch, D.-D.; Higler, R.; Hüttner, S.; Leijtens, T.; Stranks, S. D.; Snaith, H. J., High photoluminescence efficiency and optically pumped lasing in solutionprocessed mixed halide perovskite semiconductors. The Journal of Physical Chemistry Letters 2014, 5 (8), 1421-1426. 
131. Chen, K.; Barker, A. J.; Morgan, F. L.; Halpert, J. E.; Hodgkiss, J. M., Effect of carrier thermalization dynamics on light emission and amplification in organometal halide perovskites. The Journal of Physical Chemistry Letters 2014, $6(1), 153-158$.

132. Klimov, V.; Hunsche, S.; Kurz, H., Biexciton effects in femtosecond nonlinear transmission of semiconductor quantum dots. Physical Review $B$ 1994, $50(11), 8110$.

133. Klimov, V. I., Spectral and dynamical properties of multiexcitons in semiconductor nanocrystals. Annual Review of Physical Chemistry 2007, 58, 635-673.

134. Castañeda, J. A.; Nagamine, G.; Yassitepe, E.; Bonato, L. G.; Voznyy, O.; Hoogland, S.; Nogueira, A. F.; Sargent, E. H.; Cruz, C. H. B.; Padilha, L. A., Efficient biexciton interaction in perovskite quantum dots under weak and strong confinement. ACS Nano 2016, 10 (9), 8603-8609.

135. Wehrenfennig, C.; Eperon, G. E.; Johnston, M. B.; Snaith, H. J.; Herz, L. M., High charge carrier mobilities and lifetimes in organolead trihalide perovskites. Advanced Materials 2014, 26 (10), 1584-1589.

136. Savenije, T. J.; Ponseca Jr, C. S.; Kunneman, L.; Abdellah, M.; Zheng, K.; Tian, Y.; Zhu, Q.; Canton, S. E.; Scheblykin, I. G.; Pullerits, T., Thermally activated exciton dissociation and recombination control the carrier dynamics in organometal halide perovskite. The Journal of Physical Chemistry Letters 2014, 5 (13), 2189-2194.

137. Sum, T. C.; Mathews, N.; Xing, G.; Lim, S. S.; Chong, W. K.; Giovanni, D.; Dewi, H. A., Spectral features and charge dynamics of lead halide perovskites: origins and interpretations. Accounts of Chemical Research 2016, 49 (2), 294-302.

138. Neukirch, A. J.; Nie, W.; Blancon, J.-C.; Appavoo, K.; Tsai, H.; Sfeir, M. Y.; Katan, C.; Pedesseau, L.; Even, J.; Crochet, J. J., Polaron stabilization by cooperative lattice distortion and cation rotations in hybrid perovskite materials. Nano Letters 2016, 16 (6), 3809-3816.

139. Rosenwaks, Y.; Hanna, M.; Levi, D.; Szmyd, D.; Ahrenkiel, R.; Nozik, A., Hot-carrier cooling in GaAs: quantum wells versus bulk. Physical Review $B$ 1993, 48 (19), 14675.

140. Klimov, V. I.; Mikhailovsky, A. A.; McBranch, D.; Leatherdale, C. A.; Bawendi, M. G., Quantization of multiparticle Auger rates in semiconductor quantum dots. Science 2000, 287 (5455), 1011-1013.

141. Achermann, M.; Hollingsworth, J. A.; Klimov, V. I., Multiexcitons confined within a subexcitonic volume: Spectroscopic and dynamical signatures of neutral and charged biexcitons in ultrasmall semiconductor nanocrystals. Physical Review B 2003, 68 (24), 245302.

142. Kohse-Höinghaus, K.; Obwald, P.; Cool, T. A.; Kasper, T.; Hansen, N.; Qi, F.; Westbrook, C. K.; Westmoreland, P. R., Biofuel combustion chemistry: from ethanol to biodiesel. Angewandte Chemie International Edition 2010, 49 (21), 3572-3597. 
143. Antonov, I. O.; Zádor, J.; Rotavera, B.; Papajak, E.; Osborn, D. L.; Taatjes, C. A.; Sheps, L., Pressure-dependent competition among reaction pathways from first-and second- $\mathrm{O}_{2}$ additions in the low-temperature oxidation of tetrahydrofuran. The Journal of Physical Chemistry A 2016, 120 (33), 65826595.

144. Chen, M.-W.; Rotavera, B.; Chao, W.; Zádor, J.; Taatjes, C. A., Direct measurement of $\bullet \mathrm{OH}$ and $\mathrm{HO}_{2} \bullet$ formation in $\bullet \mathrm{R}+\mathrm{O}_{2}$ reactions of cyclohexane and tetrahydropyran. Physical Chemistry Chemical Physics 2018, 20 (16), 10815-10825.

145. Busch, K. W.; Busch, M. A., Cavity-ringdown spectroscopy: an ultratrace-absorption measurement technique. ACS Publications: 1999.

146. Baklanov, A. V.; Krasnoperov, L. N., Oxalyl chloride a clean source of chlorine atoms for kinetic studies. The Journal of Physical Chemistry A 2001, 105 (1), 97-103.

147. Cremer, D. t.; Pople, J., General definition of ring puckering coordinates. Journal of the American Chemical Society 1975, 97 (6), 1354-1358.

148. Cremer, D.; Pople, J. A., Molecular orbital theory of the electronic structure of organic compounds. XXIII. Pseudorotation in saturated fivemembered ring compounds. Journal of the American Chemical Society 1975, $97(6), 1358-1367$.

149. Lambert, J. B.; Papay, J. J.; Khan, S. A.; Kappauf, K. A.; Magyar, E. S., Conformational analysis of five-membered rings. Journal of the American Chemical Society 1974, 96 (19), 6112-6118.

150. Schmude Jr, R.; Harthcock, M.; Kelly, M.; Laane, J., Calculation of kinetic energy functions for the ring-puckering vibration of asymmetric fivemembered rings. Journal of Molecular Spectroscopy 1987, 124 (2), 369-378.

151. Mamleev, A.; Gunderova, L.; Galeev, R., Microwave spectrum and hindered pseudorotation of tetrahydrofuran. Journal of Structural Chemistry 2001, 42 (3), 365-370.

152. Melnik, D. G.; Gopalakrishnan, S.; Miller, T. A.; De Lucia, F. C., The absorption spectroscopy of the lowest pseudorotational states of tetrahydrofuran. The Journal of chemical physics 2003, 118 (8), 3589-3599.

153. Rayón, V. M.; Sordo, J. A., Pseudorotation motion in tetrahydrofuran: An ab initio study. The Journal of chemical physics 2005, 122 (20), 204303.

154. Hough, L., Stereochemistry of Carbohydrates: by JF Stoddart (University of Sheffield, England), Wiley-Interscience, New York, 1971, Elsevier: 1972.

155. Ionescu, A. R.; Bérces, A.; Zgierski, M. Z.; Whitfield, D. M.; Nukada, T., Conformational pathways of saturated six-membered rings. A static and dynamical density functional study. The Journal of Physical Chemistry A 2005, 109 (36), 8096-8105.

156. Stortz, C. A., Conformational pathways of simple six-membered rings. Journal of Physical Organic Chemistry 2010, 23 (12), 1173-1186. 
157. Frisch, M. J.; Trucks, G. W.; Schlegel, H. B.; Scuseria, G. E.; Robb, M. A.; Cheeseman, J. R.; Scalmani, G.; Barone, V.; Petersson, G. A.; Nakatsuji, H.; Li, X.; Caricato, M.; Marenich, A. V.; Bloino, J.; Janesko, B. G.; Gomperts, R.; Mennucci, B.; Hratchian, H. P.; Ortiz, J. V.; Izmaylov, A. F.; Sonnenberg, J. L.; Williams; Ding, F.; Lipparini, F.; Egidi, F.; Goings, J.; Peng, B.; Petrone, A.; Henderson, T.; Ranasinghe, D.; Zakrzewski, V. G.; Gao, J.; Rega, N.; Zheng, G.; Liang, W.; Hada, M.; Ehara, M.; Toyota, K.; Fukuda, R.; Hasegawa, J.; Ishida, M.; Nakajima, T.; Honda, Y.; Kitao, O.; Nakai, H.; Vreven, T.; Throssell, K.; Montgomery Jr., J. A.; Peralta, J. E.; Ogliaro, F.; Bearpark, M. J.; Heyd, J. J.; Brothers, E. N.; Kudin, K. N.; Staroverov, V. N.; Keith, T. A.; Kobayashi, R.; Normand, J.; Raghavachari, K.; Rendell, A. P.; Burant, J. C.; Iyengar, S. S.; Tomasi, J.; Cossi, M.; Millam, J. M.; Klene, M.; Adamo, C.; Cammi, R.; Ochterski, J. W.; Martin, R. L.; Morokuma, K.; Farkas, O.; Foresman, J. B.; Fox, D. J. Gaussian 16 Rev. C.01, Wallingford, CT, 2016.

158. Mozhayskiy, V. A.; Krylov, A. I. ezSpectrum.

159. Atkinson, R., A structure-activity relationship for the estimation of rate constants for the gas-phase reactions of $\mathrm{OH}$ radicals with organic compounds. International Journal of Chemical Kinetics 1987, 19 (9), 799-828.

160. Aschmann, S. M.; Atkinson, R., Rate constants for the gas-phase reactions of alkanes with $\mathrm{Cl}$ atoms at $296 \pm 2 \mathrm{~K}$. International Journal of Chemical Kinetics 1995, $27(6), 613-622$.

161. Diaz, Z.; Doepker, R. D., Gas-phase photolysis of tetrahydrofuran at 147.0 and $123.6 \mathrm{~nm}$. The Journal of Physical Chemistry 1978, 82 (1), 10-15. 162. Lee, S.-H., Dynamics of multi-channel dissociation of tetrahydrofuran photoexcited at $193 \mathrm{~nm}$ : distributions of kinetic energy, angular anisotropies and branching ratios. Physical Chemistry Chemical Physics 2010, 12 (11), 2655-2663.

163. Pushkarsky, M. B.; Zalyubovsky, S. J.; Miller, T. A., Detection and characterization of alkyl peroxy radicals using cavity ringdown spectroscopy. The Journal of Chemical Physics 2000, 112 (24), 10695-10698. 


\section{APPENDIX A}

\section{OPTICAL CAVITY ALIGNMENT AND LASERS}

\section{MAINTENANCE}

\section{A.1 CRDS Cavity alignment}

Usually, when the CRD beam is visible, the cavity is aligned using the same beam by matching the reflection of the beam from the HR coated face of the mirror with the original beam spot, indicating a perpendicular beam to the mirror (parallel to the cavity axis). However, using the same beam has some difficulties, the beam will be entirely blocked by the mirrors, where the end mirror has to be aligned first while the front mirror is unmounted to allow seeing the reflection, then one can mount the front mirror and align it independently, in this case one cannot see the reflection of the end mirror since it is already blocked by the front mirror, and walking the cavity becomes completely dependent on ring down curve is seen on the oscilloscope which in most of the cases is not obtained immediately. This method becomes even more difficult in the case of invisible laser beams - like IR laser beam - using the same beam since reflection needs an IR viewer or IR card. Using a visible reference beam is very useful to overcome such difficulties. 
The visible reference beam must be chosen based on the center of HR mirrors, where the mirrors are transparent to the reference beam, so its reflections still visible even when both mirrors are mounted. A HeNe laser beam is the best choice for such purpose, however, in some cases when the center of the mirrors is close to HeNe wavelength and can cover broad region - depending on the coating and IR region of interest, HeNe laser might be entirely blocked by the mirrors, in this case using a green laser pointer or other laser source away from the center of the mirrors is useful.

Below is a complete ring-down cavity alignment we developed based on our experimental setup. Note that mirrors and optical elements are labeled according to the CRD beam path; the definitions of optics can be varied based on different experimental setups.

Our ring-down cavity is aligned using a HeNe laser beam $(632.8 \mathrm{~nm})$ unidirectional with the CRD laser beam. It is critical to make sure that both beams are $100 \%$ overlapping before the cavity. This is done by using a pair of Irises and mirrors just before the cavity. Also, one wants to make sure that beams go through the center of the cavity, where the CRD mirrors are perpendicular to the reference HeNe laser beam, i.e., the axis of the cavity. Then, since the IR beam overlaps with the reference beam, the IR beam will be automatically aligned when the reference beam is aligned. The alignment procedure has many steps, as described below. All steps are based on figure A.1 and must be followed strictly to achieve the maximum 
ring-down time and signal level. Some of the steps below are specific for our setup. General steps are the same for any experimental setup. One can ignore those steps that are irrelevant to his setup. The optical arrangement can vary from setup to another, while the general procedure remains the same.

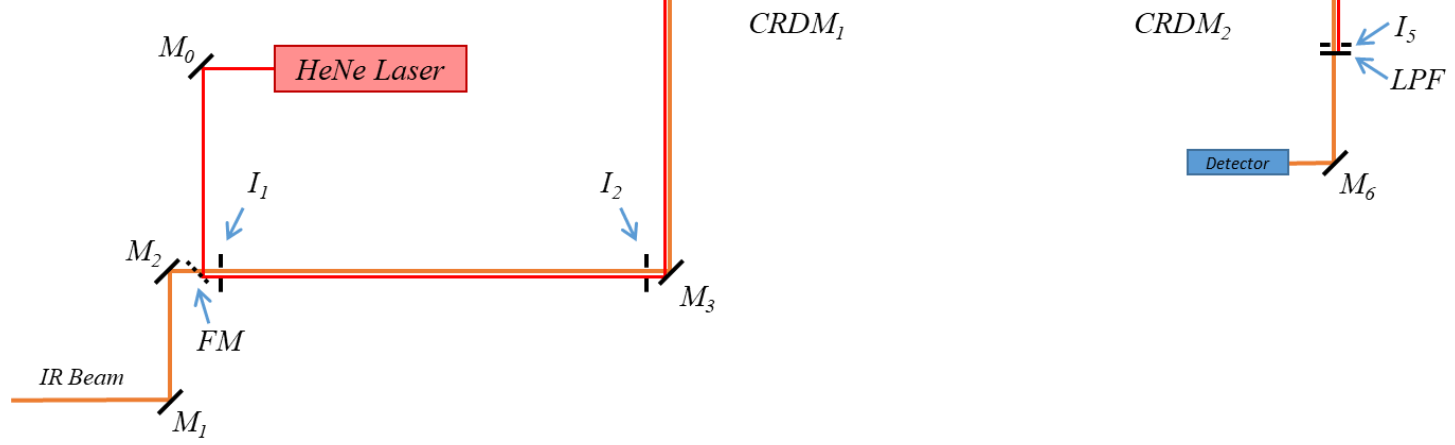

Figure A.1. Beam paths diagram for both IR and HeNe lasers. Beams are shifted for clarity. (CRDM) Cavity Ring Down Mirror, (FM) Flipping Mirror, (I) Iris, (M) Mirror, (LPF) Long Pass Filter.

The first step in the alignment process must be aligning the CRD beam to the optical cavity, i.e., the CRD cavity should be the reference point for the whole alignment process. The height of the beams must be adjusted based on the cavity height. All irises are defined after aligning the CRD beam to the center axis of the cavity.

1. CRD laser beam alignment to the cavity center axis. 
This process is not necessary for the everyday experiments. It should be performed when the CRDS system needs major re-alignment, e.g., when the CRD laser beam is re-aligned significantly, or optical elements changed.

\section{Important!}

- Use a beam dumber to block the laser beams just before the detector to avoid detector damage.

- Use minimum visible and IR laser power for alignment. Especially in the case of the green laser beam.

- Guide all beams using the holes on the optical table. This makes the process easier.

- When setting up irises, fix them as close as possible to mirrors. This will make sure the corresponding mirror will not change the beam alignment much on the closer iris.

1.1. Unmount the CRD mirrors from the cavity. Flip down the flip mirror if it is already installed in the setup.

1.2. Use four mirrors before the cavity to direct the beam towards the cavity, and 1 or 2 mirrors after the cavity to direct the beam to the detector. The beam path is shown in figure A.1. Note that the arrangement of the four mirrors could vary from setup to another based on available space on the optical table.

Mirrors are labeled $\mathrm{M}_{1}-\mathrm{M}_{6}$ in figure A.1, two mirrors $\left(\mathrm{M}_{1}\right.$ and $\left.\mathrm{M}_{2}\right)$ are needed for aligning the beam in the "overlap area" between $\mathrm{M}_{2}$ and $\mathrm{M}_{3}$, the 
second pair of mirrors $\left(M_{3}\right.$ and $\left.M_{4}\right)$ will be used for aligning the beam to the center axis of the cavity between Mirrors $\mathrm{M}_{4}$ and $\mathrm{M}_{5}$, while Mirrors $\mathrm{M}_{5}$ and $\mathrm{M}_{6}$ are used to align the beam to the detector.

1.3. Direct the IR beam to the cavity, make sure the beam is horizontal (parallel to the optical table), and match the height of the center axis of the cavity. one can use a ruler to measure the heights. Make sure the beam hits the center of the
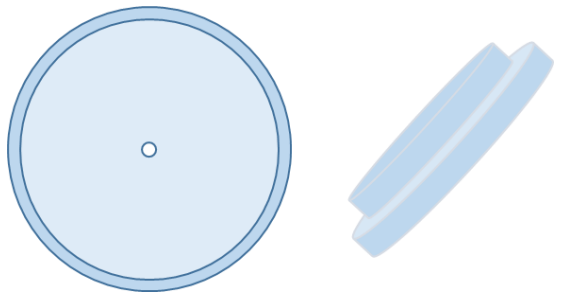

Figure A.2. Beam alignment mirrors; this will give more freedom for the further alignment steps. tool machined using an acrylic disk to match the cavity arm end.

Steps $1.4-1.6$ are to define the center axis of the cavity

1.4. Use a beam alignment tool, a plastic disk that fits into the opening of each arm of the cavity cell or chamber and has a small round hole at the center for the laser beam to go through (Figure A. 2), to define the beam into the center axis of the ring-down cavity. At this stage, one needs to use an IR card or viewer to be able to see the laser beam. 
1.5. Put the cavity alignment tool into the arm opening closer to Iris $\mathrm{I}_{3}$ (lefthand side in Figure A.1). Steer the IR laser beam through the center hole of the tool using Mirror $\mathrm{M}_{3}$.

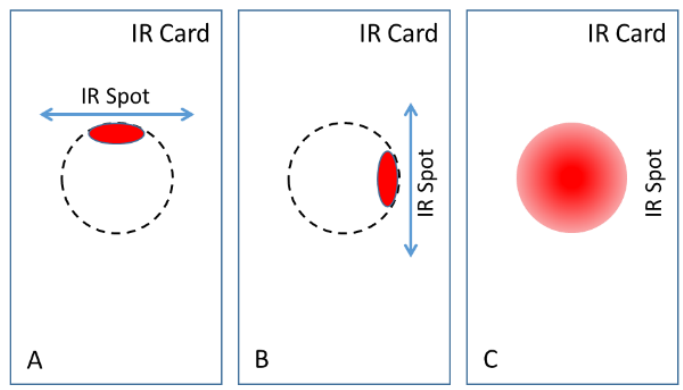

Figure A.3. Alignment of the IR spot on the IR card using the Beam

1.6. Put the cavity alignment tool into alignment tool, dashed line is the foot print of the IR spot if it is the arm opening closer to $\mathrm{Iris}_{4} \mathrm{I}_{4}$ rightcentered on the axis of the cavity and iris $\mathrm{I}_{4} . \quad \mathrm{A}$ is the vertical hand side in Figure A.1). Steer the IR centering, B is the horizontal laser beam through the center hole of centering and $\mathrm{C}$ is aligned spot.

the tool using Mirror $\mathrm{M}_{4}$. Use an IR viewer to visualize the IR beam on the beam alignment tool hole. For better alignment, one may use an IR card, move the IR laser beam vertically (horizontally) by adjusting the Mirror $\mathrm{M}_{4}$ so that only a very small portion of the beam goes through the center hole of the tool (Figure A.3). One then adjusts the

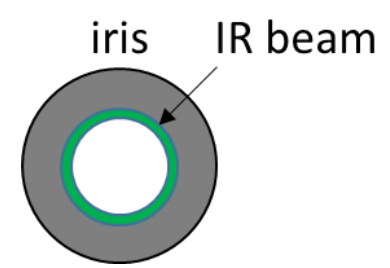

Figure A.4. Visualizing Mirror $\mathrm{M}_{4}$ horizontally (vertically) to the IR spot on the Irises using an IR viewer. the make sure that the IR laser beam is green ring is what is seen when the IR beam is centered horizontally (vertically). One centered on the iris and finally moves Mirror $\mathrm{M}_{4}$ vertically the iris is partially closed. (horizontally) to the center. 
1.7. If necessary, repeat steps 1.5 and 1.6 until the IR laser beam is centered at both ends.

1.8. Use mirror $\mathrm{M}_{3}$ to center the beam on the front cavity end and mirror $\mathrm{M}_{4}$ for the rear cavity end, repeat the steps until the beam is centered on both ends. Now, the IR beam is aligned to the center axis of the cavity.

1.9. Adjust the position and height of Irises $\mathrm{I}_{3}$ and $\mathrm{I}_{4}$ so that the $\mathrm{HeNe}$ laser beam is centered on both irises. The center axis of the cavity is now defined by Irises $\mathrm{I}_{3}$ and $\mathrm{I}_{4}$.

1.10. Define irises $I_{1}, I_{2}$, and $I_{5}$ based on the IR beam. Make sure to leave some space between mirror $\mathrm{M}_{3}$ and Iris $\mathrm{I}_{3}$ to setup the flip mirror. Now all irises are defined based on the center axis of the cavity. Use an IR viewer to look at the IR laser beam at Irises while defining them. (See Figure A.3, seen through an IR viewer).

2. Alignment of the HeNe laser beam:

This step must take place after aligning the IR beam since the HeNe Laser beam is aligned based on the irises defined in the previous step. HeNe must be aligned in the center of irises $\mathrm{I}_{1}$ and $\mathrm{I}_{2}$. Once the HeNe is Centered on these irises, it will be colinear with the IR beam. The HeNe laser beam should be automatically centered on the Irises $I_{3}-I_{5}$, and in the center axis of the cavity since all irises are positioned based on the IR beam. (see steps $1.4-1.9)$ 


\section{Important!}

- Block the IR beam before mirror $\mathrm{M}_{2}$ for safety.

- For the HeNe Alignment, use ONLY mirror $\mathrm{M}_{0}$ and the flip mirror FM; otherwise, steps $1.4-1.8$ needs to be redone.

2.1. Setup at least two mirrors, including the flip mirror, to steer the HeNe laser beam. The flip mirror will intercept the IR beam just before iris $\mathrm{I}_{1}$.

2.2. Make sure that the He-Ne laser beam is centered at Iris $\mathrm{I}_{1}$ and Iris $\mathrm{I}_{2}$. Use mirror $\mathrm{M}_{0}$ to center the HeNe beam on iris $\mathrm{I}_{1}$ and use FM to center the HeNe beam on iris $\mathrm{I}_{2}$.

2.3. Repeat step 2.2 until the HeNe laser is centered on irises $\mathrm{I}_{1}$ and I2. This makes sure that the HeNe laser beam overlaps with the IR laser beam afterward. Now both beams are overlapping. Any optical alignment for HeNe will be automatically reflected on the IR beam.

When the center axis of the cavity is already defined by irises $I_{3}$ and $I_{4}$, and both IR and HeNe beams are overlapping, steps $2.4-2.9$ is applied for detector alignment.

2.4. Steer the He-Ne laser beam to the center of Iris $\mathrm{I}_{3}$ using Mirror $\mathrm{M}_{3}$.

2.5. Steer the He-Ne laser beam to the center of Iris $\mathrm{I}_{4}$ using Mirror $\mathrm{M}_{4}$. 
2.6. If necessary, repeat steps 2.4 and 2.5 until the HeNe laser beam is centered at both irises $\mathrm{I}_{3}$ and $\mathrm{I}_{4}$. Now, the $\mathrm{IR}$ beam is following the HeNe beam; any alignment on the HeNe will be reflected on the IR beam.

2.7. Unblock the IR beam (keep the detector blocked), use a power sensor just before the cavity and measure the IR power. Usually, $\sim 3$ $\mathrm{mJ}$ is sufficient for the CRDS experiment. Decrease the IR laser power to minimum detectable. One can also use an IR viewer. The IR power should be decreased so much that it can be barely seen with the IR viewer. Remove the black cardboard in front of the photodiode.

2.8. Adjust Mirror $\mathrm{M}_{5}$ to maximize the signal ( $\sim 10 \mathrm{~ns}$ pulse duration) on the oscilloscope. If cw laser is used, one can set up a chopper for better visualization on the scope. The detection system (Iris $I_{5}$, LongPass Filter, and the photodiode) should be parallel to the IR beam and at the same height for the max signal.

3. Alignment of the ring-down cavity:

The IR beam should be blocked for Steps 3.2-3.14.

3.1. Make sure that both beams (IR and HeNe laser beams) are centered on irises $I_{1}$ and $I_{2}$, i.e., both beams are overlapping.

3.2. Steps $2.4-2.6$ are applied here. 
3.3. Place the cavity alignment card, a white card that has several pinholes with different diameters, between Mirrors $\mathrm{M}_{3}$ and $\mathrm{M}_{4}$. Adjust its position so that the HeNe laser beam goes through a hole that is slightly smaller than the laser beam and is perfectly centered.

3.4. The rear CRD mirror (further from the HeNe laser, CRD Mirror 2 in Figure A.1) can now be mounted with the high-reflectivity (HR) coated surface inside of the cavity. If only one wedged CRD mirror for the cavity, it should be used at the front end to minimize the deflection of the HeNe laser beam (see Step 4.1).

3.5. Use the adjustment screws of $\left(\mathrm{CRDM}_{2}\right)$ mirror mount to steer the reflection of the HeNe laser beam from the coated surface back to the used pinhole on the beam alignment card. The reflection should be perfectly centered at the pinhole so that a ring around it can be seen.

If CRDM is wedged, two reflection beam spots, from two surfaces of the CRD mirror, can be seen. Usually, the reflection from the HR surface is stronger and larger. To identify the reflection beam spots, one can rotate the mirror along the axis of the ring-down cavity. The reflection from the HR surface is almost 
stationary, while the other reflected beam spot, by the other surface of the mirror, rotates around the first reflected beam (See Figure A.5).

3.6. One now can see several rings around the reflected beam from the HR surface of the CRD mirror on the beam alignment tool. If they are not co-centered, use mirror $\mathrm{M}_{4}$ to co-center them (Figure A.6a). This makes sure that the $\mathrm{HeNe}$ beam hits the Figure A.6. Ring structure seen on the cavity alignment card. (A) comparasion between centered and off-centered reflection, (B) picture of centered ring structure of reflection.

center of CRD mirror 2.

Very often, the ring structure couldn't be observed. In this case, a single circular spot of the reflection on the alignment card should be observed, see figure A.7. If the spot was not circular, move the beam horizontally and vertically using mirror $\mathrm{M}_{4}$ until one sees a defined circular reflection.

3.7. If the rings were not co-centered (or the reflection spot was not circular), then step 3.6 slightly moves the center position of the reflection. Therefore, Step 3.5 need to be repeated to achieve a perfect co-centered beam pattern around the pinhole on the beam alignment card (Figure A.6b). 
3.8. Once the rear CRD mirror is aligned, the front mirror (closer to the HeNe laser, CRD Mirror $\mathrm{CRDM}_{1}$ in Figure A.1) can be mounted with the HR coated surface inside of the cavity.

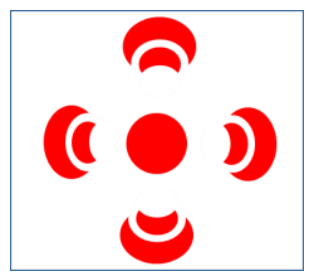

Figure A.7. Reflection spot if ring structure was not observed.

3.9. Use the adjustment screws of $\left(\mathrm{CRDM}_{2}\right)$ mirror mount to steer the reflection of the HeNe laser beam from the coated surface back very close to the used pinhole on the beam alignment card. When both CRDMs are mounted, one can see not only the direct reflection from the HR surfaces of CRD Mirrors 1 and 2 but also the second-round reflection: The transmitted portion of the laser beam from $\mathrm{CRDM}_{1}$ will be reflected by $\mathrm{CRDM}_{2}$, a portion of the second reflection will be transmitted again through $\mathrm{CRDM}_{1}$ to the beam alignment card. The second-round reflection beam spot is smaller and weaker than the direct reflection spots. One may see the third- or even fourth-round reflection, depending on the intensity of the HeNe laser beam.

3.10. Use the adjustment screws of CRD mirrors 1 and 2 to align all three reflection beams vertically (see Figure A.8a). Reflection from 
CRD Mirror 1 is centered at the pinhole. The distance between the centers of the direct reflection spots should approximately equal to the distance between the

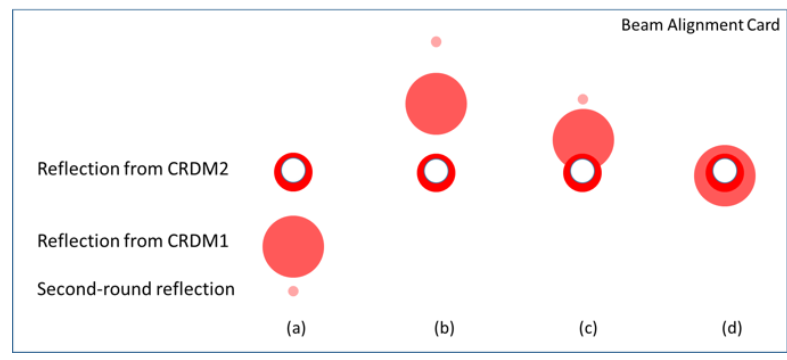

Figure A.8. First and second round reflections when both CRDM are mounted. the second reflection is twice further than the first and travels twice faster.

centers of the CRD Mirror 2 reflection and the second-round reflection. One can use the vertical adjustment screw of CRD Mirror2 to move its own direct reflection beam spot and the second-round reflection beam spot vertically, above and below the pinhole (Figure A.8a and $8 b)$. These two configurations should be symmetric to each other.

If not, adjust "walk" the vertical adjustment screws of both CRD mirrors alternately to achieve symmetry.

3.11. After the symmetry is achieved, use the vertical adjustment screw of CRD Mirror 2 to overlap all reflection beams spots with the pinhole.

3.12. Place a white card after the cavity. A ring-structure interference pattern may be observed. Optimize the interference pattern by adjusting the adjustment screws of the two CRD mirrors alternately. 
4. Ring-down cavity Fine adjustments.

4.1. Flip down the flip mirror. The HeNe laser can now be blocked. If the aforementioned preliminary optimization steps are all well performed, a ring-down curve should be immediately observed on the oscilloscope.

4.2. If the ring-down curve contains many high-frequency oscillations, multiple modes in the transmitted beam are detected. One can narrow irises along the IR beam path and/or slightly adjust Mirror 5 to eliminate the multi-mode signal. Note that M5 should not be adjusted significantly at this stage. Over-adjustment it may mislead the following adjustment steps.

Steps 5.3-5.8 are to fine adjust the ring-down cavity. Multi-exponential fit in the LabVIEW program should be selected (usually with two components), and one should monitor the ring-down time of the slowest component (longer ring down time).

The goal of optimization should be to increase the ring-down time, not to increase the magnitude of the ring-down signal. Indeed, when ring-down time increases, the magnitude decreases because the transmission is stretched in time;

4.3. Adjust the vertical adjustment screw of CRD Mirror 1 to increase the ring-down time. 
4.4. The ring-down time can be further increased by "walking" the vertical adjustment screws of both CRD mirrors: one adjusts the vertical screw of one of the CRDS mirrors, and then adjust the vertical screw of the other CRD mirror to increase the ring-down time. If the optimized signal has longer ring-down time than previous, one should continue adjusting the first mirror in the same direction; Otherwise, one reverses the direction of adjustment, until the maximum ring-down time is reached.

4.5. Walk all adjustment screws of the CRD mirrors to increase the ring-down time. Always walk the corresponding screws of the two mounts.

4.6. Walk Mirrors 3 and 4 to increase the ring-down time.

4.7. Repeat Steps 4.5-4.7 to maximize the ring-down time.

A good ring-down signal should have long ring-down time, large magnitude, and smooth decay curve, which implies a single mode. The beam profile of the transmitted CRD laser beam can be illustrated by a CCD camera. Single TEM00 mode is desired.

4.8. A telescope and 50 or $100 \mu \mathrm{m}$ diameter pinhole may be added at the con-focal point of the telescope, for mode selection.

It is essential to follow all steps strictly. If one does not perform each and every step accurately, one might be trapped in a local maximum. When the global maximum is reached, all ring-down mirror adjustment screws 
become very sensitive. It is recommended to lock these screws for stability and reproducibility, especially when the vacuum pump is running.

\section{A.2 Excimer laser LPX120i maintenance.}

a. Changing mirrors

Mirrors need to be changed if the operation wavelength of the laser is changed, i.e., gases and ration. For example, when changing from 248 to 193.

- Fill the laser with helium slightly above atmospheric pressure (say 1.05 - $1.1 \mathrm{~atm})$, although the extra pressure will leak out once mirrors are removed. However, it will limit humidity and unwanted gases from entering the chamber.

- Immediately seal the laser tube with a rubber stopper.

- If the rear mirror is transparent, then you can easily align the cavity with helium-neon laser, however, if only mirrors were removed but the mounts kept on the laser, there should be no change in cavity alignment.

- After changing mirrors, re-passivation is required, and helium burn is recommended.

Do both Helium Burn and Re-passivation when (i) there is a leak in the gas line or the laser tube, or (ii) the laser tube is exposed to ambient air, e.g., after mirror cleaning, or (iii) switching from $\mathrm{F}$ to $\mathrm{Cl}$ and vice versa.

b. Helium burns 
- Connect Helium tank to "Inert".

- Go to "Menu", bring the "Helium Burn" tag to the front. HV should be 16-18 kV. A low voltage of $16 \mathrm{kV}$ is preferred. The repetition rate should be $3 \mathrm{~Hz}$. Helium pressure should be 1600 mbar. All other gas pressures should be zero.

- Quit Menu and go back to the main menu.

- Make sure that both the big and small valves of the Helium tank are open.

- Make sure that "Option>Low Light" is chosen. Otherwise, the laser will stop firing because it cannot detect any laser beam.

- Go to Gas >New Fill. Refill the laser tube. It will stop automatically at slightly above 1600 mbar. Quit Gas and go back to the main menu.

- Press "Start".

The gas chamber needs to be evacuated, refilled, and Helium burn started repetitively. For each round, one needs to choose "Gas $>$ New Fill" and then "Start".

Gradually increase the repetition rate after each refill, e.g., one may start with a low repetition rate $(5 \mathrm{~Hz})$ to a high repetition rate $(25 \mathrm{~Hz})$. Begin with a time duration of $\sim 10$ minutes and then gradually increase the duration of laser-firing, one may use the following process:

First run $5 \mathrm{~Hz}, 16 \mathrm{kV}$, for $10 \mathrm{~min}$;

Second run $10 \mathrm{~Hz}, 16 \mathrm{kV}$, for $20 \mathrm{~min}$; 
Third run $15 \mathrm{~Hz}, 16 \mathrm{kV}$, for $30 \mathrm{~min}$;

Fourth run $20 \mathrm{~Hz}, 16 \mathrm{kV}$, for $30 \mathrm{~min}$;

Fifth run $25 \mathrm{~Hz}, 16 \mathrm{kV}$, for $30 \mathrm{~min}$;

Sixth run $25 \mathrm{~Hz}, 16 \mathrm{kV}$, for $30 \mathrm{~min}$;

Seventh run $25 \mathrm{~Hz}, 16 \mathrm{kV}$, for 30 min or longer.

To test if no further helium burns are needed, put a white paper close to the output coupler to see the color of the discharge. First, the discharge looks white, then, it turns pink, the standard color of Helium discharge.

c. Re-passivation

Re-passivation is recommended when the laser tube is warm, for example, immediately after the Helium burn.

Stage (1) Soaking:

Soaking has to be done manually. First, add 150 mbar of $5 \% \mathrm{~F}_{2}$ in $\mathrm{He}$. Then add 1,200 mbar Helium. Then, leave the laser overnight. Stage (2) Re-passivation.

- Connect Helium tank to "Inert" and $\mathrm{F}_{2}$ in Helium to "halogen".

- Go to "Menu", bring the "Re-passivation" tag to the front. HV should be $16 \mathrm{kV}$. The repetition rate should be $5 \mathrm{~Hz}$. However, if an external trigger is chosen in the menu, the external trigger repetition rate overrules. $\mathrm{F}_{2}$ in Helium pressure should be 30 mbar. Helium pressure should be 2000 mbar. All other gas pressures should be zero.

- Quit Menu and go back to the main menu. 
Make sure that both the big and small valve of both the Helium and the F2 in Helium tanks are open. Also, make sure that "Option>Low Light" is chosen. Otherwise, the laser will stop firing because it cannot detect any laser beam.

- Go to Gas $>$ New Fill. Refill the laser tube. The software will add 30 mbar of $5 \% \mathrm{~F}_{2}$ and 2000 mbar Helium. It will stop automatically at slightly above 2030 mbar.

- Quit Gas and go back to the main menu.

- Press "Start".

The laser will fire at $16 \mathrm{kV} \mathrm{HV}$ and $5 \mathrm{~Hz}$ repetition rate. Put a white paper at the exit. One should see a red beam. If one sees arcing, stop the firing immediately. Evacuate the gases, let the laser cool down, and refill it with less $\mathrm{F}_{2}$. If it still arc, increase the $\mathrm{HV}$ to 17 or $18 \mathrm{kV}$ and see if the arcing is still there.

At the first fill, the red color fades out very quickly. When it fades out, add a little bit more 5\% fluorine ( 20 mbar). ("Bump" the fluorine.) 
Repeat "bumping" the fluorine until the red color lasts for more than 10,000 laser shots.

When the red color lasts for more than 10,000 laser shots, stop adding $5 \% \mathrm{~F}_{2}$. Instead, evacuate the laser tube and refill it with $30 \mathrm{mbar} 5 \% \mathrm{~F}_{2}$ and 2,000 (please confirm) mbar Helium.

After several refills, the red color should stay for longer than 90,000 laser shots. Refill the laser about ten times at the 90,000 shots level. The laser is now ready. 


\section{APPENDIX B}

\section{PYTHON PROGRAMS FOR DATA ANALYSIS}

\section{First, parameters extraction and initial calculations:}

Usually, we use gaussian for our quantum chemical calculations. Hence, this program was coded to handle Gaussian output files only. The first run will ask the user for several necessary information, including; scaling factors, temperature, branching ratios (previously calculated by SAR, see above) if more than one isomer exists. Then it looks up the files to extract the necessary parameters from Gaussian output files of both ground and excited states using "keywords", these keywords will ensure the extraction of the final optimized values. The values extracted from the ground state files include Hartree-Fock energies, and then, it zero-point corrects these energies, then it extracts the optimized rotational constants. From the excited state file, similarly to the ground state, it extracts optimized rotational constants, zero-point corrected Hartree-Fock energies vertical excitations, and electron dipole moments. The adiabatic excitations and oscillator strengths are calculated using the parameters extracted from both ground and excited states. All the above-extracted parameters are along with calculated quantities that are then sorted and stored in a table. 
Once all conformers are done, Boltzmann distribution and branching ratios are calculated saved as a text file to be used in the next step of the program. A schematic diagram shows the structure chart of the first subprogram is shown in figure B.1a.

Second, Franck-Condon factors (FCFs) calculations

FCFs calculations are done by eZspectrum software. This software requires an input file to be prepared before running the software. This python subprogram prepares the input file of eZspectrum software using the table generated above and Gaussian output file similarly to the first step above and then calls the eZspectrum software to perform FCFs calculations. A stick plot will be generated and saved in a text file for each conformer to be used in the last step. A schematic diagram shows the structure chart of the second subprogram is shown in figure B.1b.

\section{Third, spectra convolution with Gaussian or Lorentzian line shapes}

After necessary parameters and quantities are collected and calculated, and the stick plots generated in the previous step, the third program will generate convoluted spectra for each conformer and generate overall spectra using the superposition of all conformers weighted by their populations 
generated in the first step. Figure B.1c shows the structure chart of the third step program.

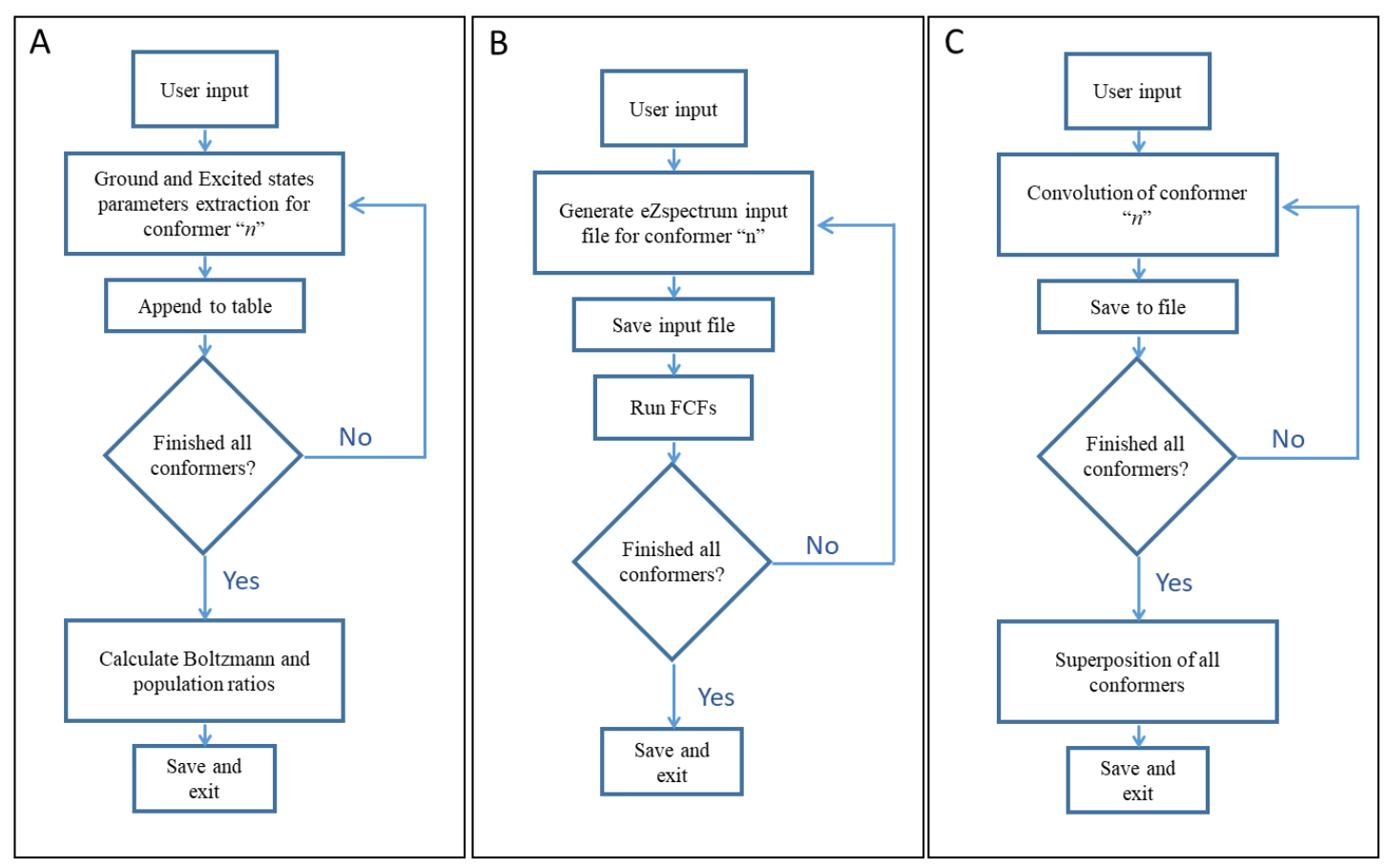

Figure B.1. Flow chart for the three python programs used in data analysis, gaussian parameter extraction (A), FCFs Calculations (B) and spectra convolution (C). 
Python code:

The code was written using Python 3.7 environments. It is specific to the experiments conducted in our lab. However, it can be modified if needed.

Prerequisites:

Python 3 environment has to be installed on the computer. The program eZspectrum.exe should be in the same folder that contains Gaussian output files and the atomic masses.xml associated with the eZspectrum program. All files will be generated in the same folder.

The gaussian output file has to be ".log" extension, or the code can be modified to ".out" and the naming of the files should follow the following criteria:

A first character is an integer number indicating the isomer number. The second character is a lower-case letter indicating different conformers, and the last character has to be an upper-case letter indicating its electronic state. In this code, "X" is used for the ground state, "A or B" for the excited state. However, these criteria can be changed on the code as well.

In the second step program, the code between the yellow-highlighted areas is a modified code of the associated XML file generation that comes with the eZspectrum software package (reference [158]). 


\section{First step code:}

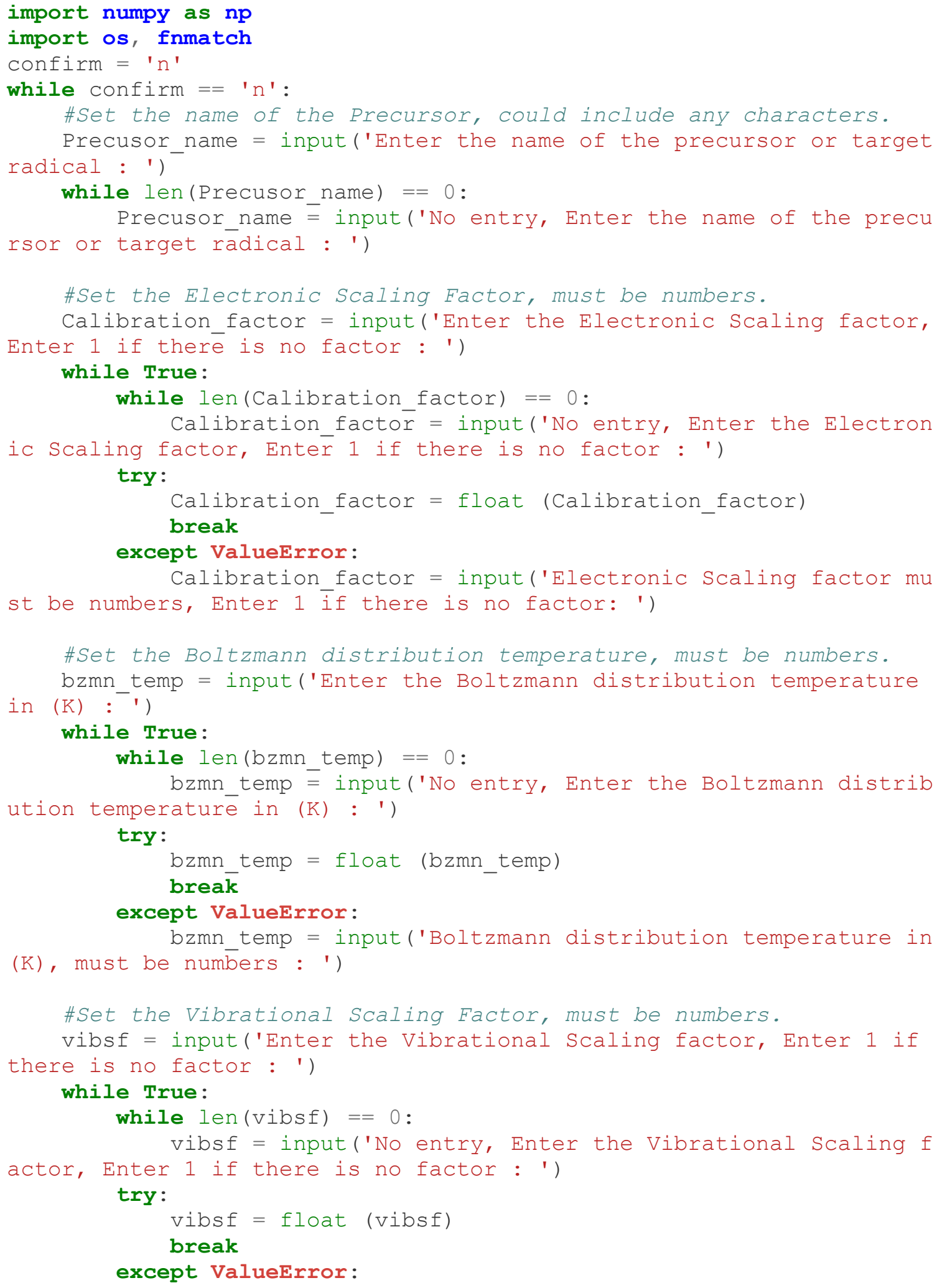


vibsf = input('Vibrational Scaling factor must be number

s, Enter 1 if there is no factor: ')

\#Set the isomers, must be numbers

numberofisomers = input('Enter the number of isomers : ')

while True:

while len (numberofisomers) $==0$ :

somers : ') numberofisomers = input('No entry, Enter the number of $i$

try:

numberofisomers = int (numberofisomers)

break

except ValueError:

numberofisomers = input('Input must be integers, please enter the number of isomers: ')

\# Set branching ratio for each isomer.

if int (numberofisomers) $>1$ :

Branching_ratio $=n p \cdot z e r o s(i n t(n u m b e r o f i s o m e r s)$ )

for $i$ in range (int (numberofisomers)) :

isomernumber $=\operatorname{str}(i+1)$

branchingRatio = input('Enter the branching ratio for is

omer ' + isomernumber + ' : ')

while True:

while len (branchingRatio) $==0$ :

branchingRatio = input('No entry, Enter the bran

ching ratio for isomer '+isomernumber+' : ')

try:

Branching_ratio[i] = branchingRatio

break

except ValueError:

a number, please re-enter: ')

branchingRatio = input ('Branching ratio must be

else:

Branching_ratio $=1$

use_f = input('Do you want to use the oscillator strength? Y or $\mathrm{N}:$ : )

while len (use f) $==0$ :

use_f = input('No entry, Enter if you want to use the oscill

ator strength? Y or $\mathrm{N}$ : ')

exit1 = ' $\mathrm{n}$ '

while exit1 $==$ ' $n$ ':

if (use_f $=={ }^{\prime} Y^{\prime}$ ) or (use_ $f=={ }^{\prime} \mathrm{Y}^{\prime}$ ) or (use_f $==\mathrm{A}^{\prime}$ ) ) or (us e_f $==$ 'n' $\left.^{\prime}\right)$ :

else:

exit1 $=$ ' $y$ '

use $f=$ input('Enter if you want to use the oscillator s trength? must be $\mathrm{Y}$ or $\mathrm{N}$ : ' ')

exit1 $=$ ' $n$ '

confirm = input('please confirm the above parameters, type $y$ if correct, $\mathrm{n}$ if need to change: ') 


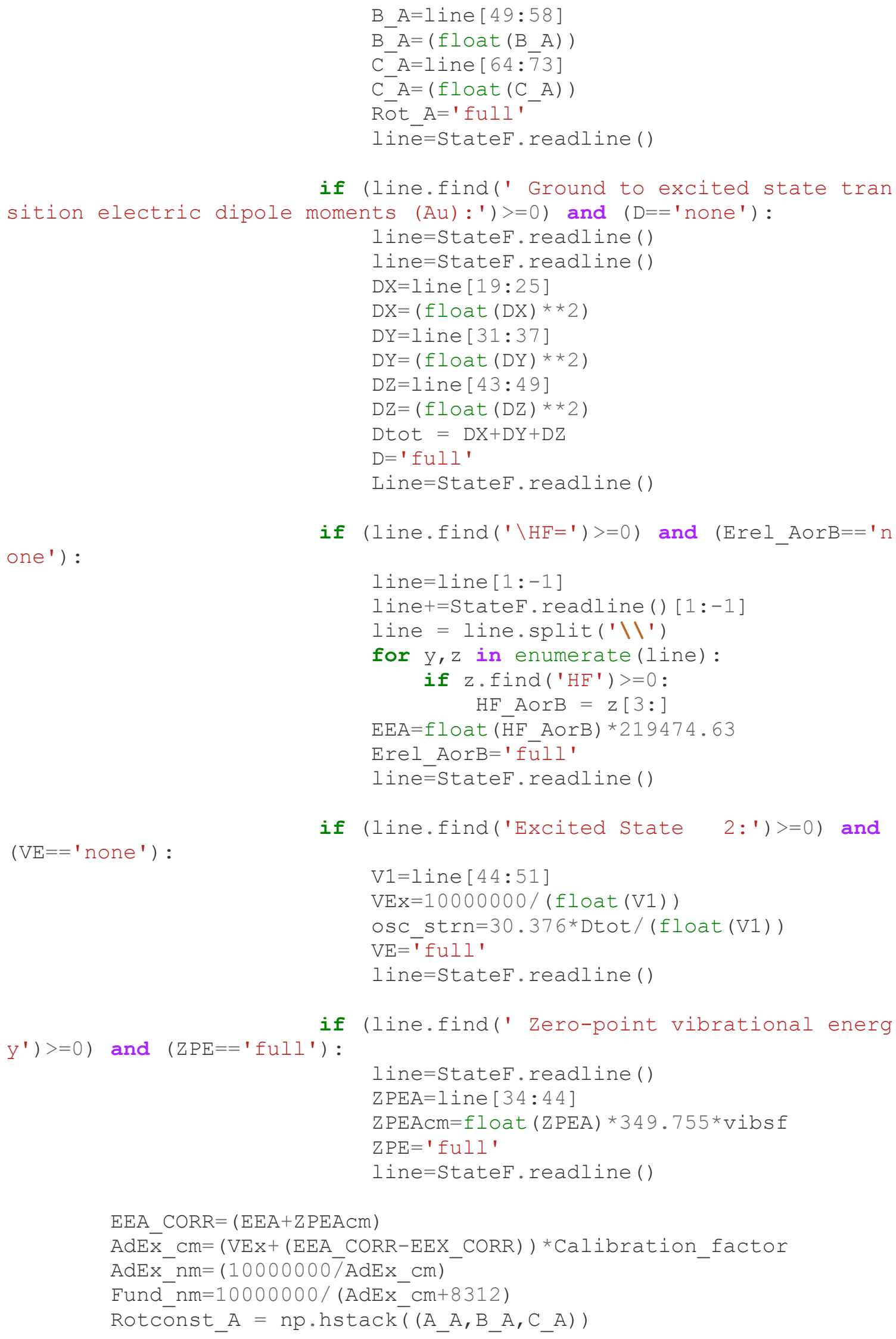




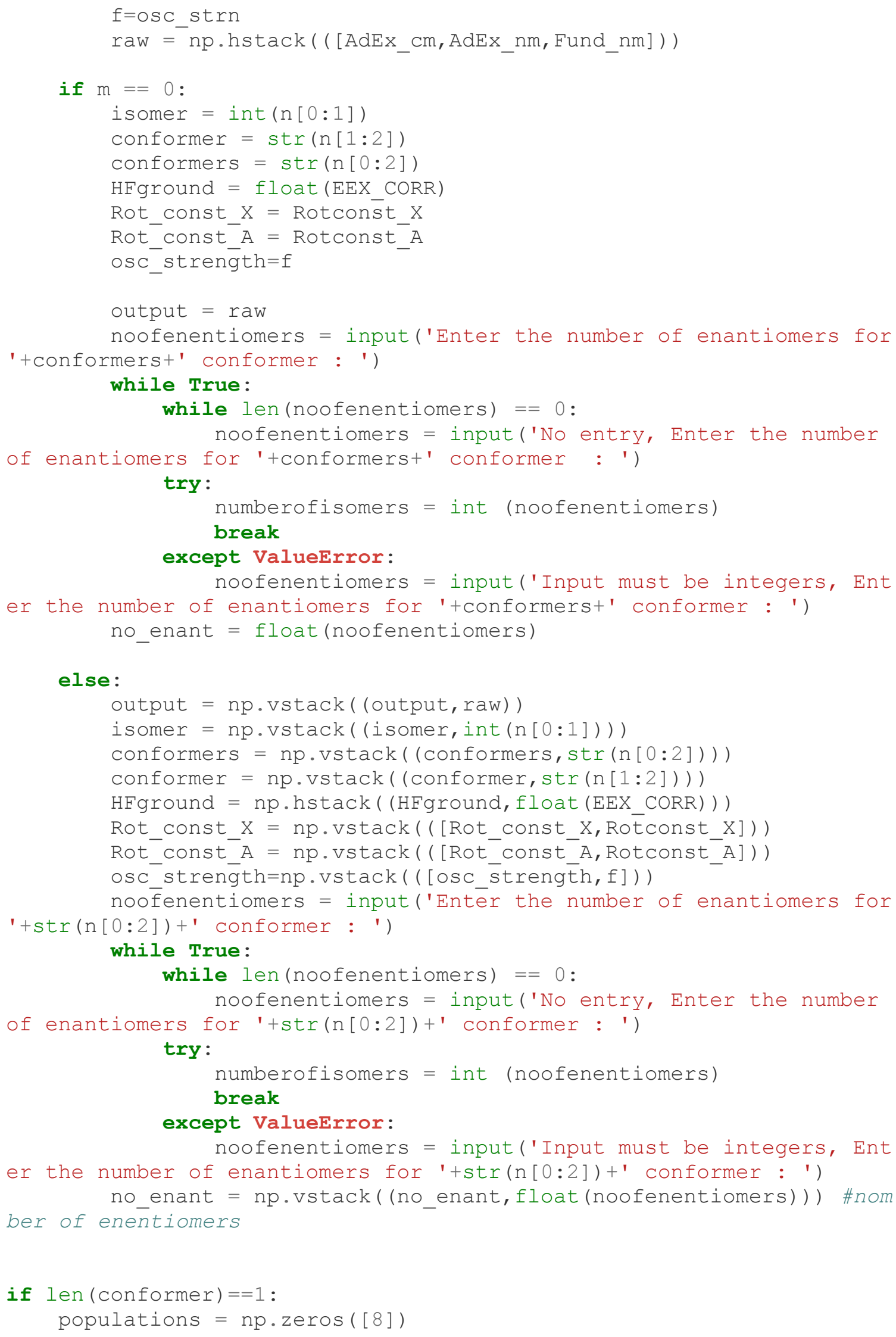




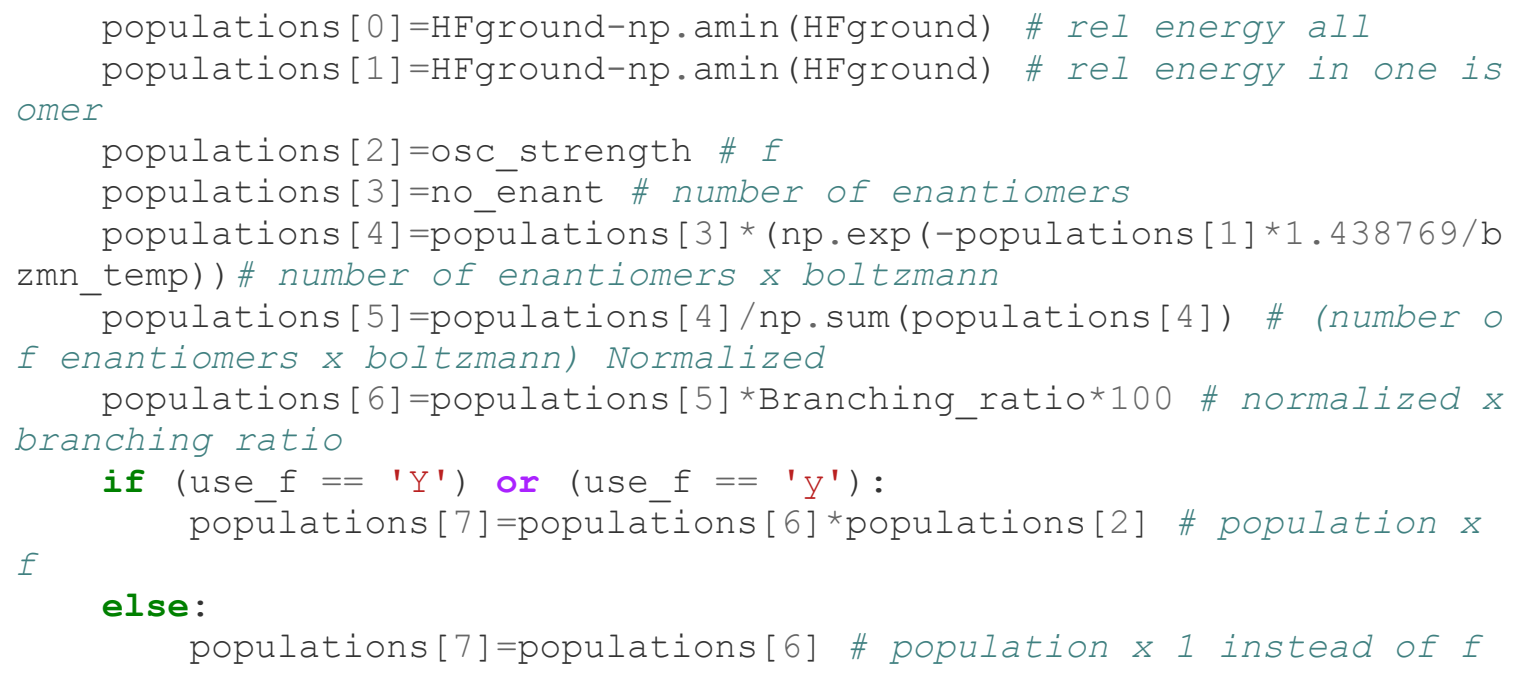




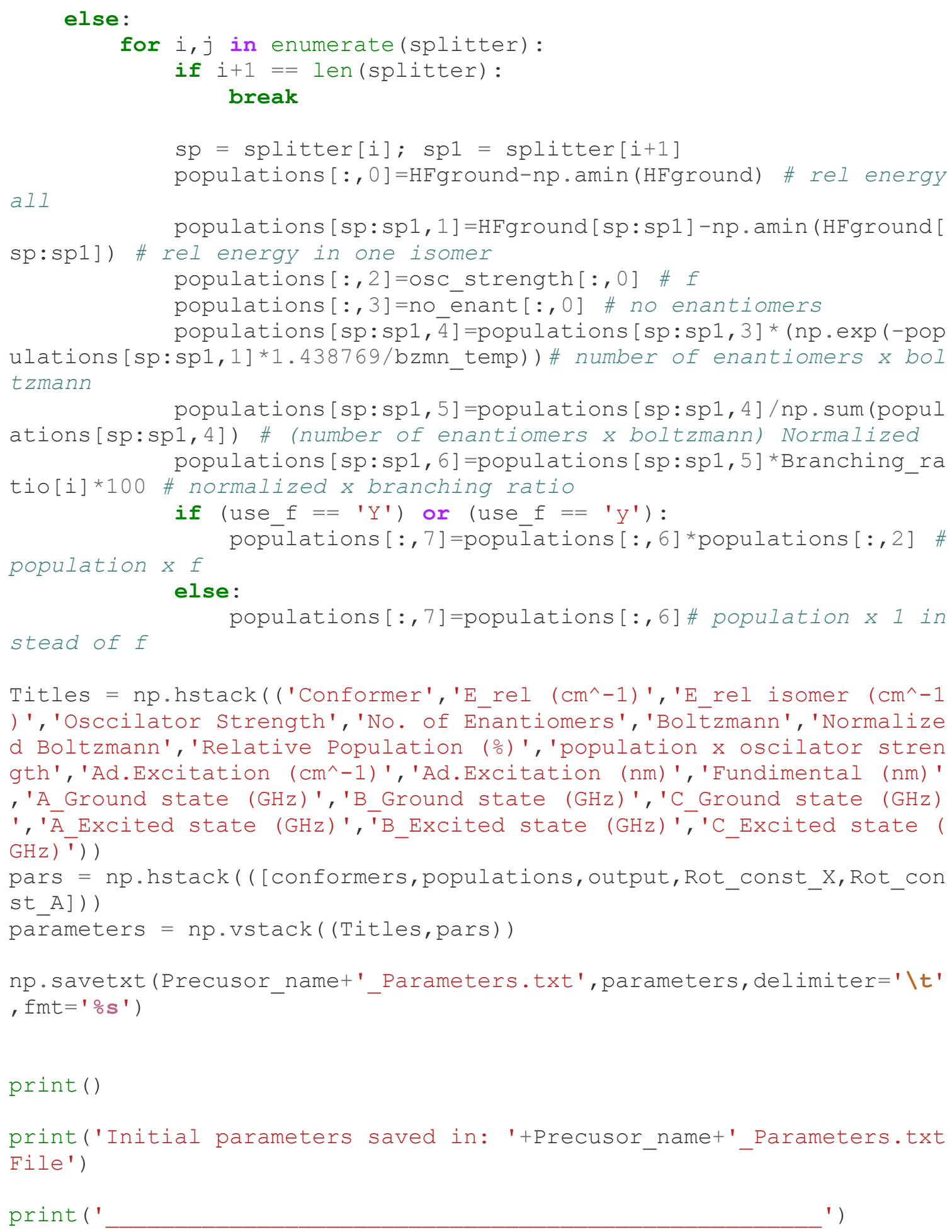




\section{second step code:}

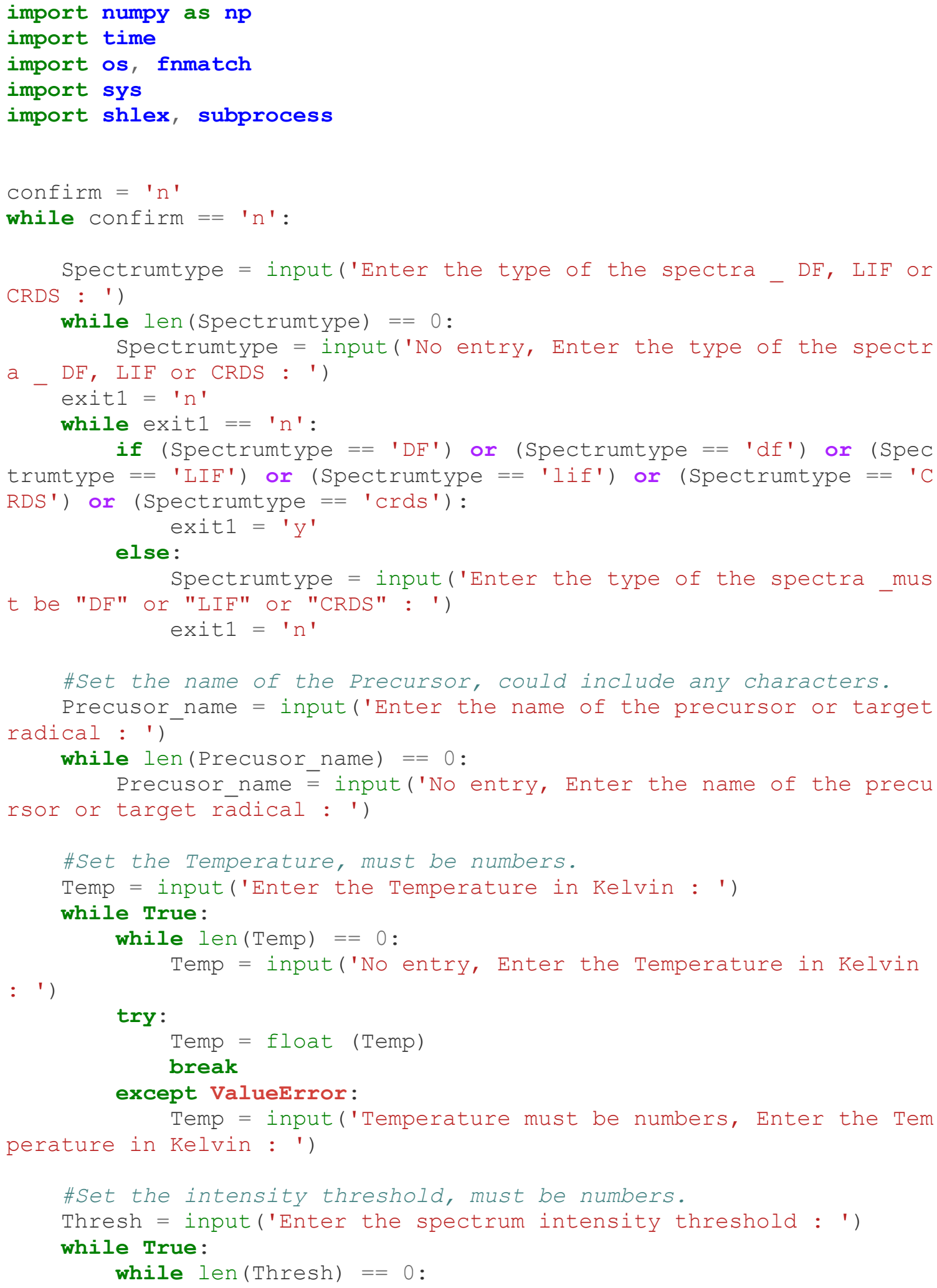




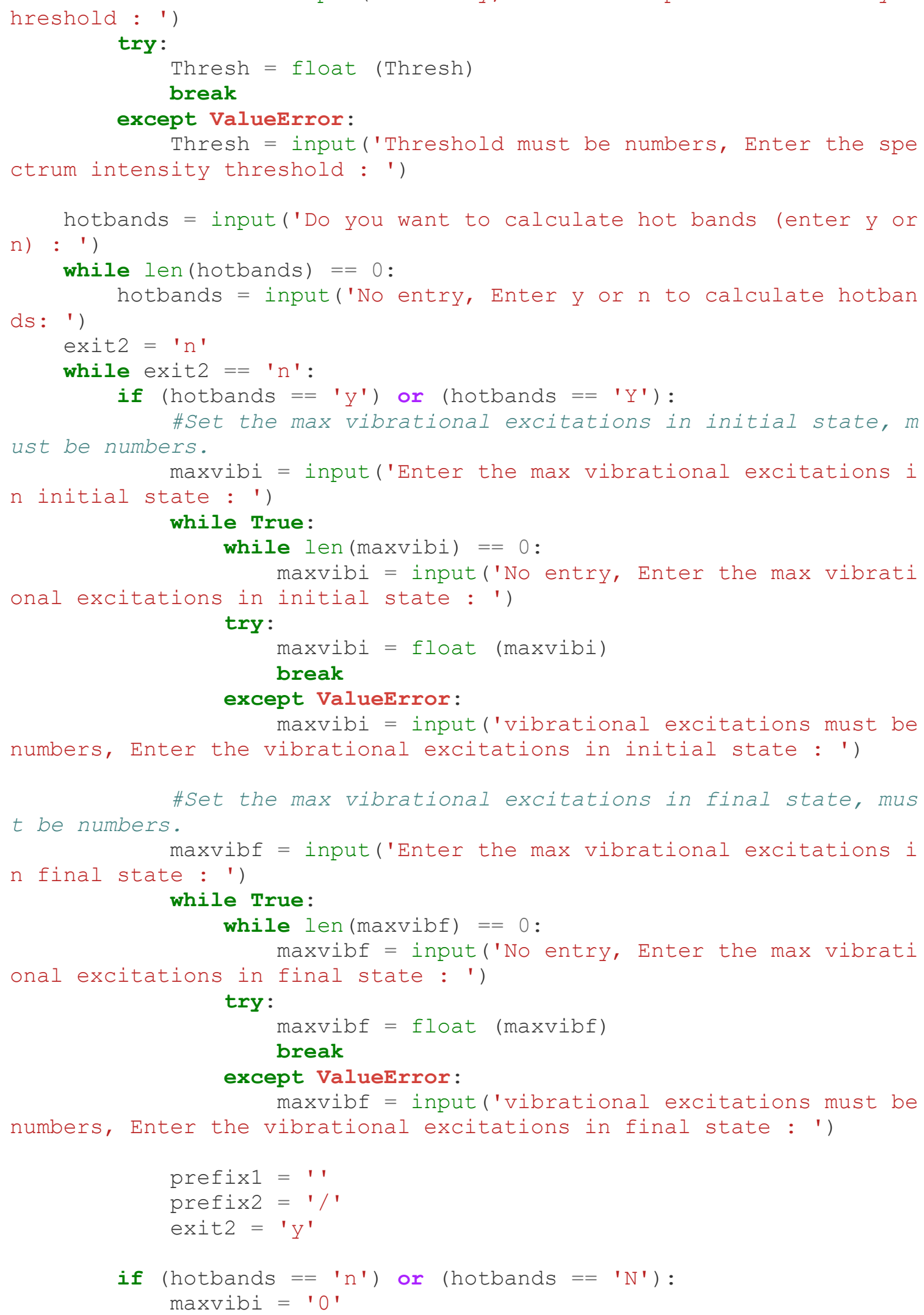


n final state : ')

maxvibf = input('Enter the max vibrational excitations $i$

while True:

while len (maxvibf) $==0$ :

maxvibf = input('No entry, Enter the max vibrati

onal excitations in final state : ')

try:

maxvibf = float (maxvibf)

break

except ValueError:

maxvibf = input ('vibrational excitations must be numbers, Enter the vibrational excitations in final state : ')

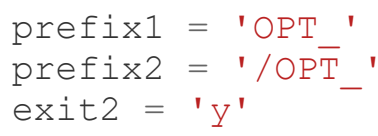

confirm = input('please confirm the above parameters, type y if correct, $\mathrm{n}$ if need to change: ')

while len (confirm) $==0$ :

confirm = input('No entry, confirm the above parameters, typ e $y$ if correct, $n$ if need to change: ')

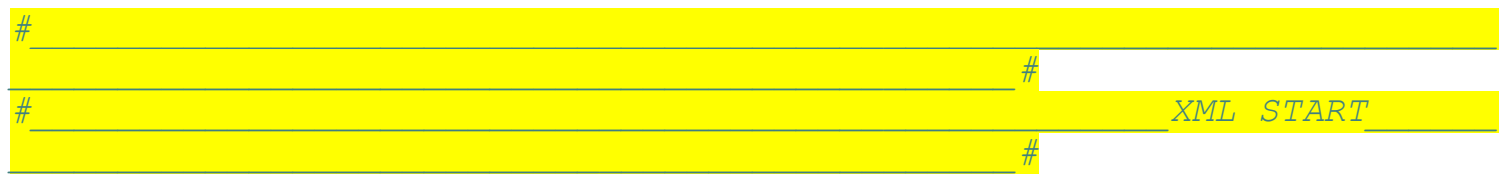

def XML (xml_filename, ai_filenames, run_type):

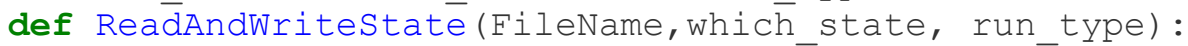

\# $=====>$ read parameters from input files

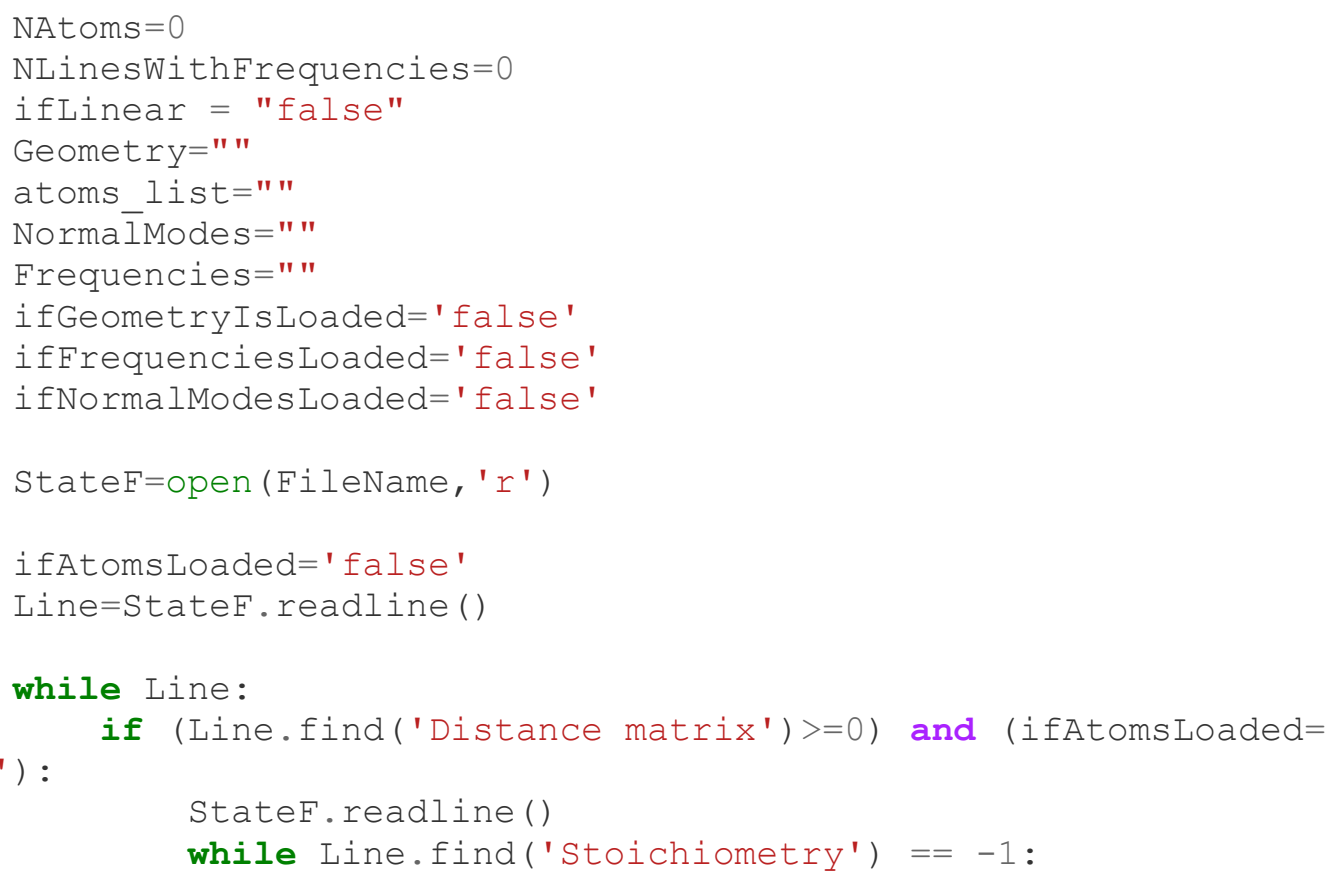




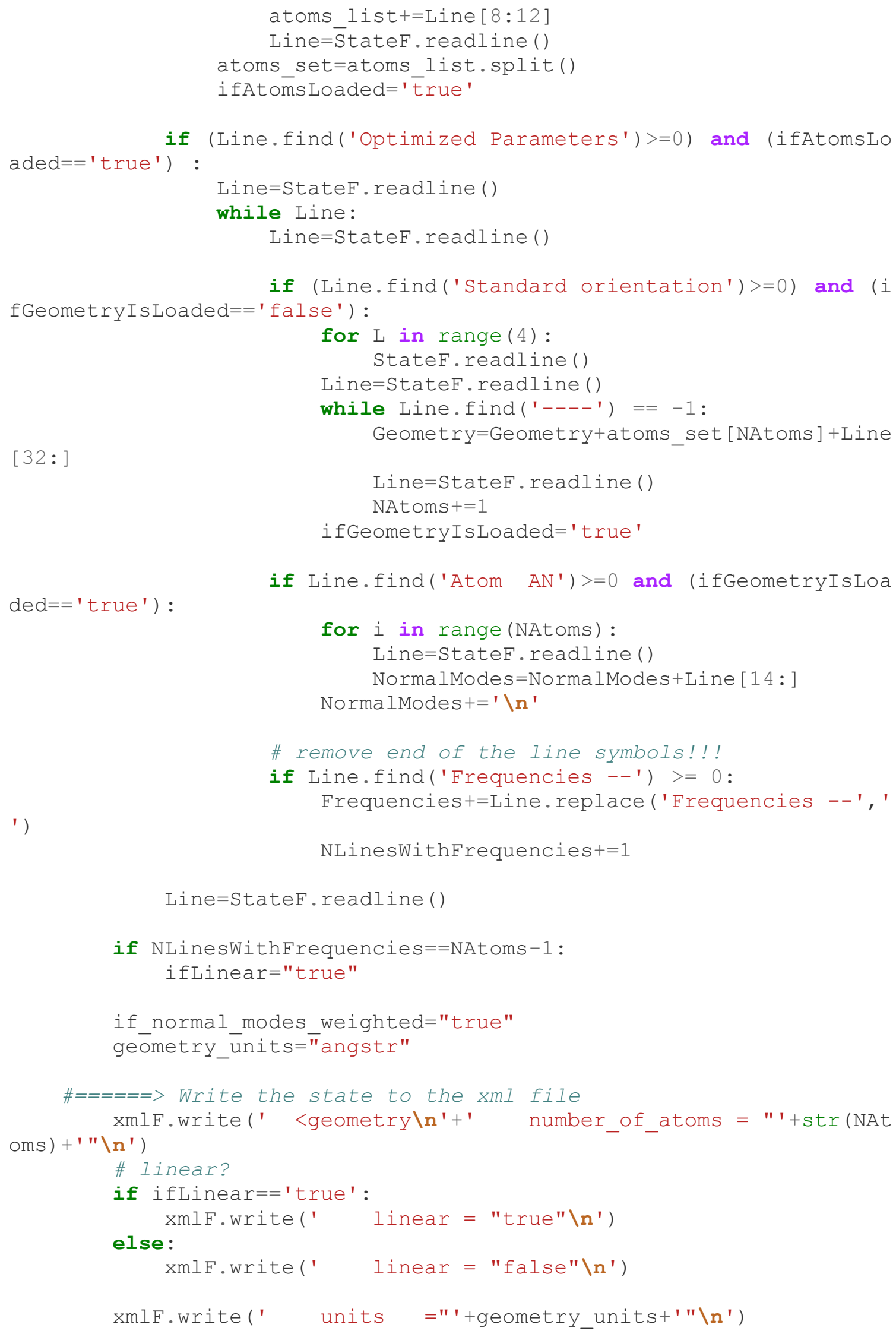




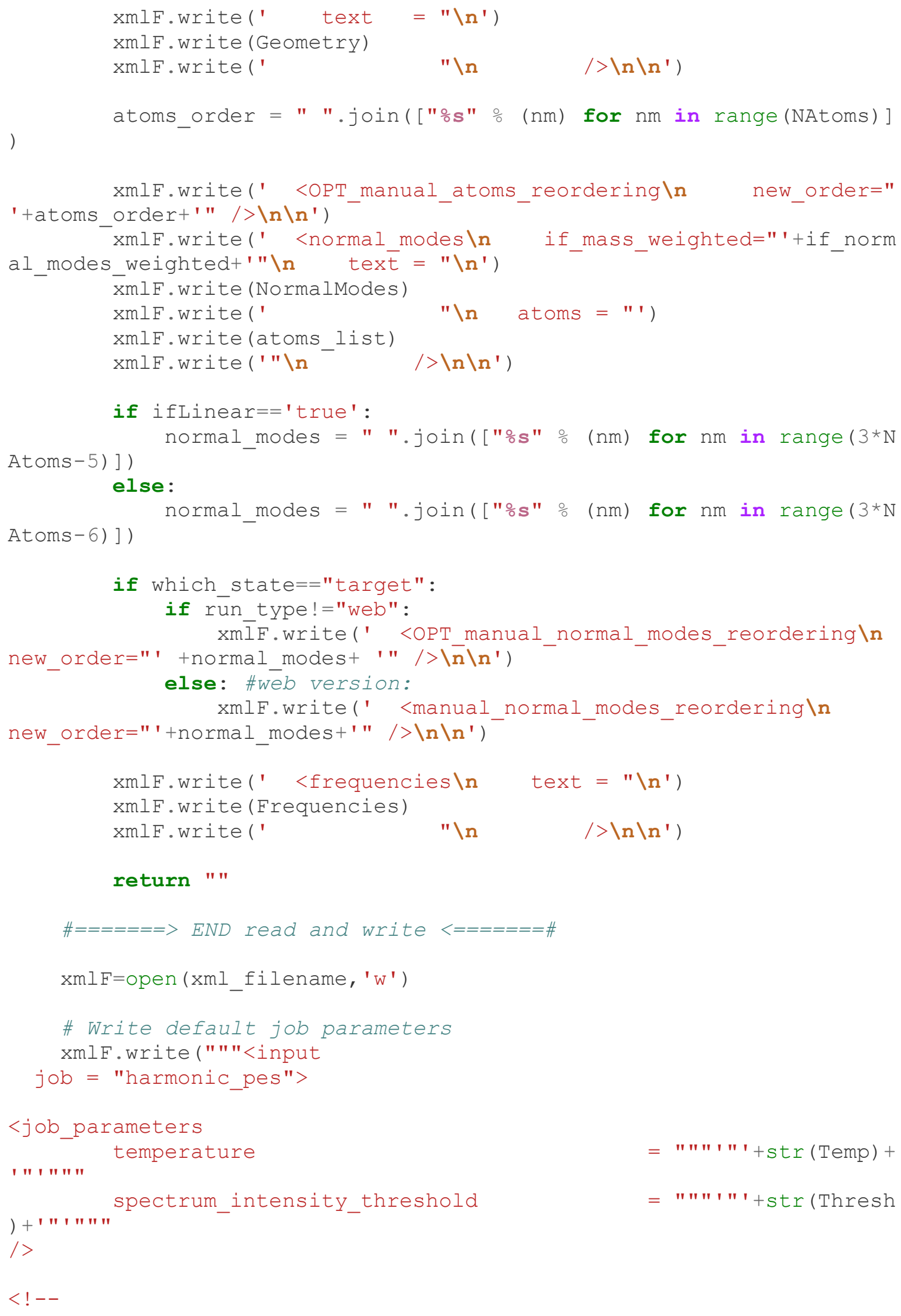


Tags which start with "OPT " will be ignored.

To include these optional keywords please "uncomment" by removin 9

"OPT_" from the start and the corresponding end tag (if present)

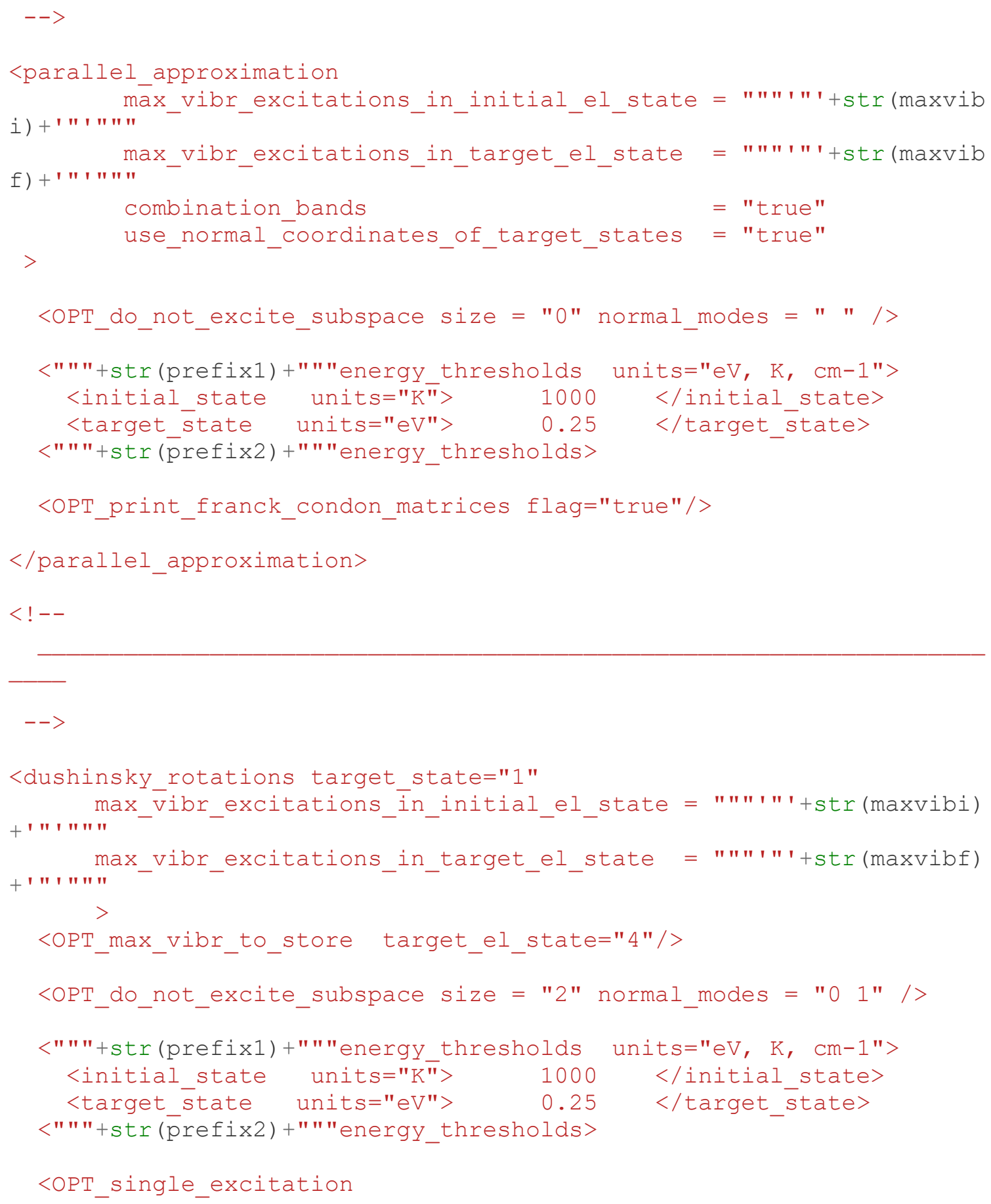




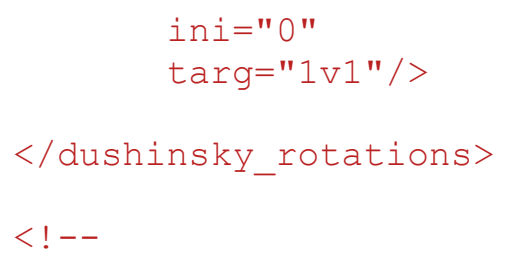




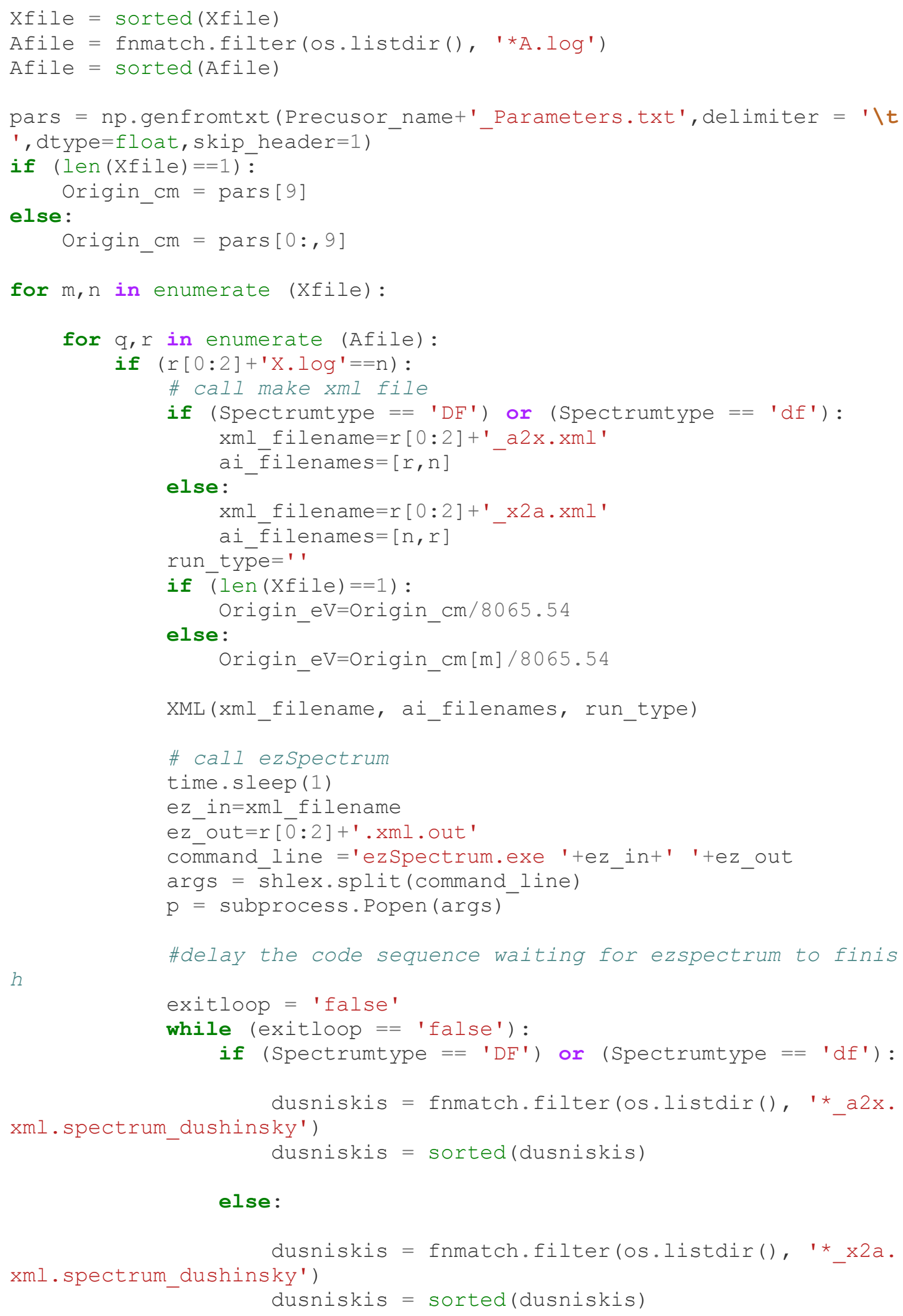




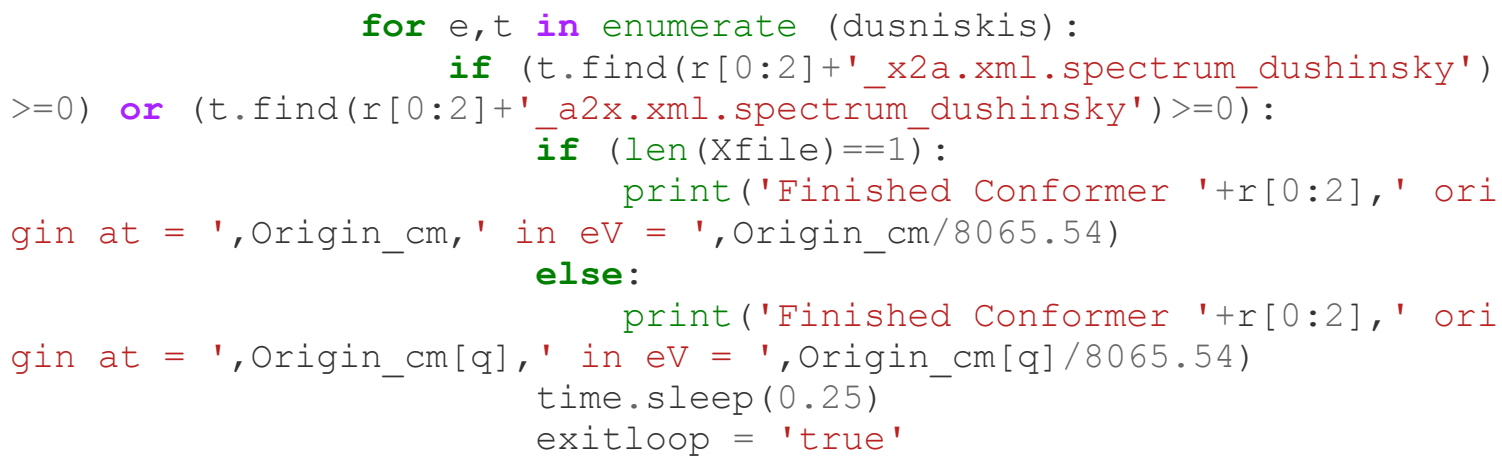

\section{Third step code:}

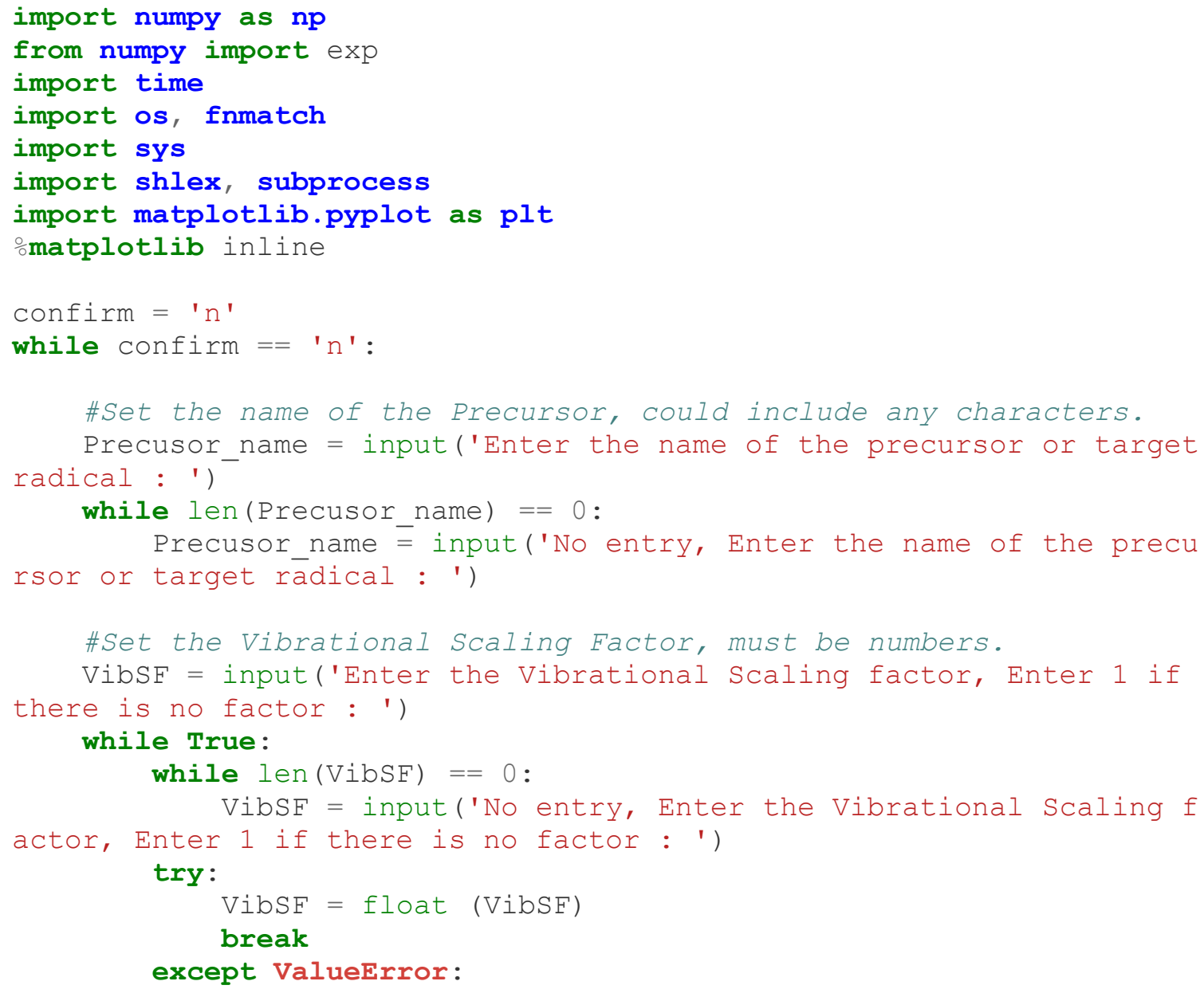


VibSF = input('Vibrational Scaling factor must be number

s, Enter 1 if there is no factor: ')

\# Set the function used in simulation

L : ')

func = input('select line shape, Gaussian = G, for Lorentzian =

while len (func) $==0$ :

func = input('No entry, select line shape, Gaussian= G, for

Lorentzian $=\mathrm{L}:$ ')

exit1 = ' $n$ '

while exit1 == ' $n$ ':

if (func $==$ ' $^{\prime}$ ') or (func $=={ }^{\prime} g^{\prime}$ ) or (func $==$ or' $^{\prime}$ ) or (func $\left.==l^{\prime}\right):$

else:

$$
\text { exit1 }=\text { ' } y \text { ' }
$$

func = input ('invalid entry, line shape must be "G" or "

L" ' )

$$
\text { exit1 = ' } n \text { ' }
$$

func $=\operatorname{str}($ func $)$

\# Set the simulated spectra step size, must be numbers.

')

stepsize = input('Enter the stepsize in spectrum in wavenumber:

while True:

while len (stepsize) $==0$ :

stepsize = input('No entry, Enter the stepsize in spectr um in wavenumber : ')

try:

stepsize = float (stepsize)

break

except ValueError:

stepsize = input('stepsize must be numbers, Enter the st epsize in spectrum in wavenumber : ')

\# Set the simulated spectra linewedth, must be numbers.

segma = input('Enter the linewedth in spectrum in wavenumber : '

)

\section{while True:}

while len ( segma) $==0$ :

in wavenumber : ') segma = input('No entry, Enter the linewedth in spectrum

try:

segma $=$ float $($ segma $)$

break

except ValueError:

segma = input('Linewedth must be numbers, Enter the line wedth in spectrum in wavenumber : ')

\# Set the magnification order of magnitude, must be numbers. orderofmagnitude = input('Enter the order of magnitude for the $\mathrm{m}$ agnification factor : ')

while True:

while len (orderofmagnitude) $==0$ : 


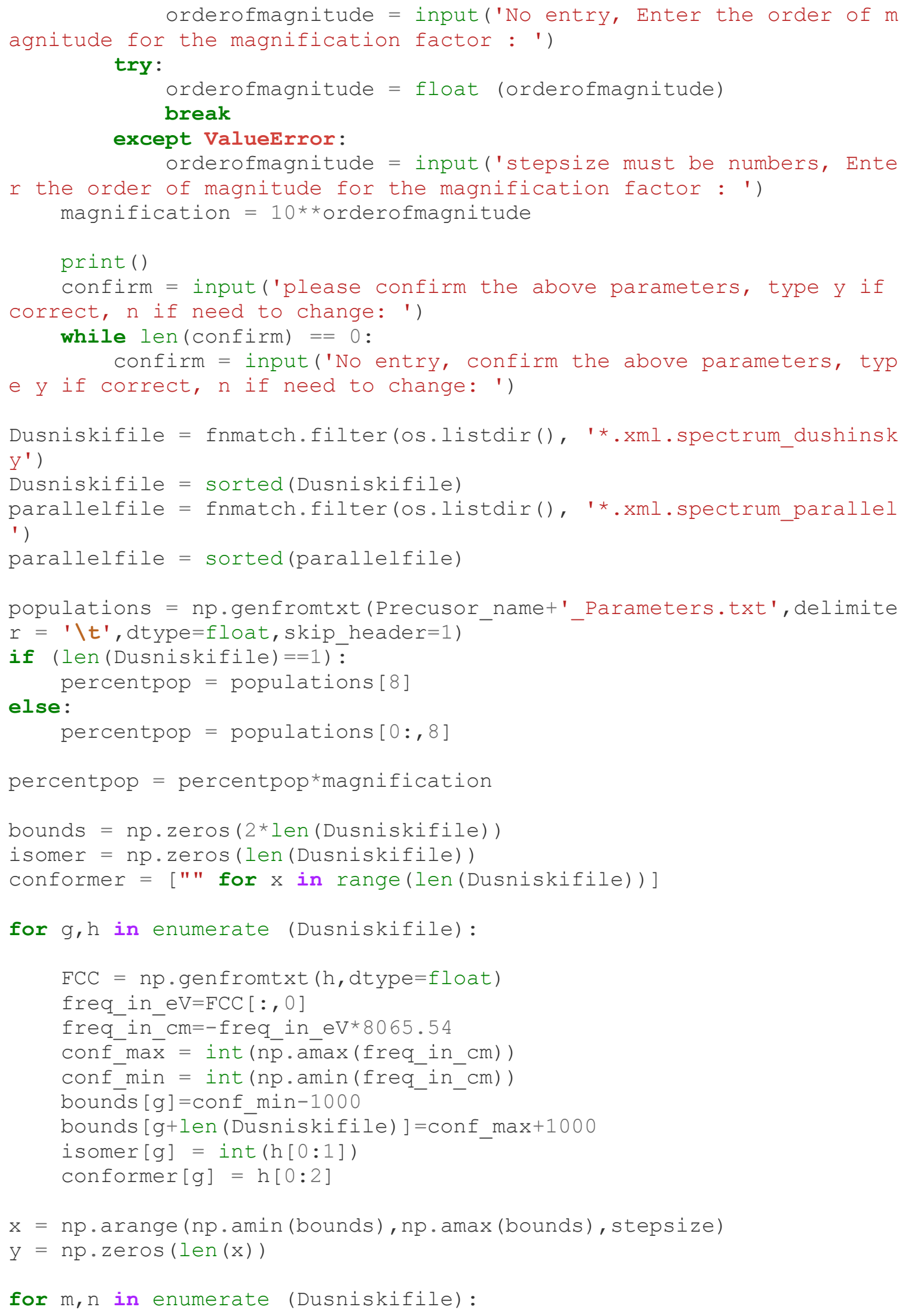




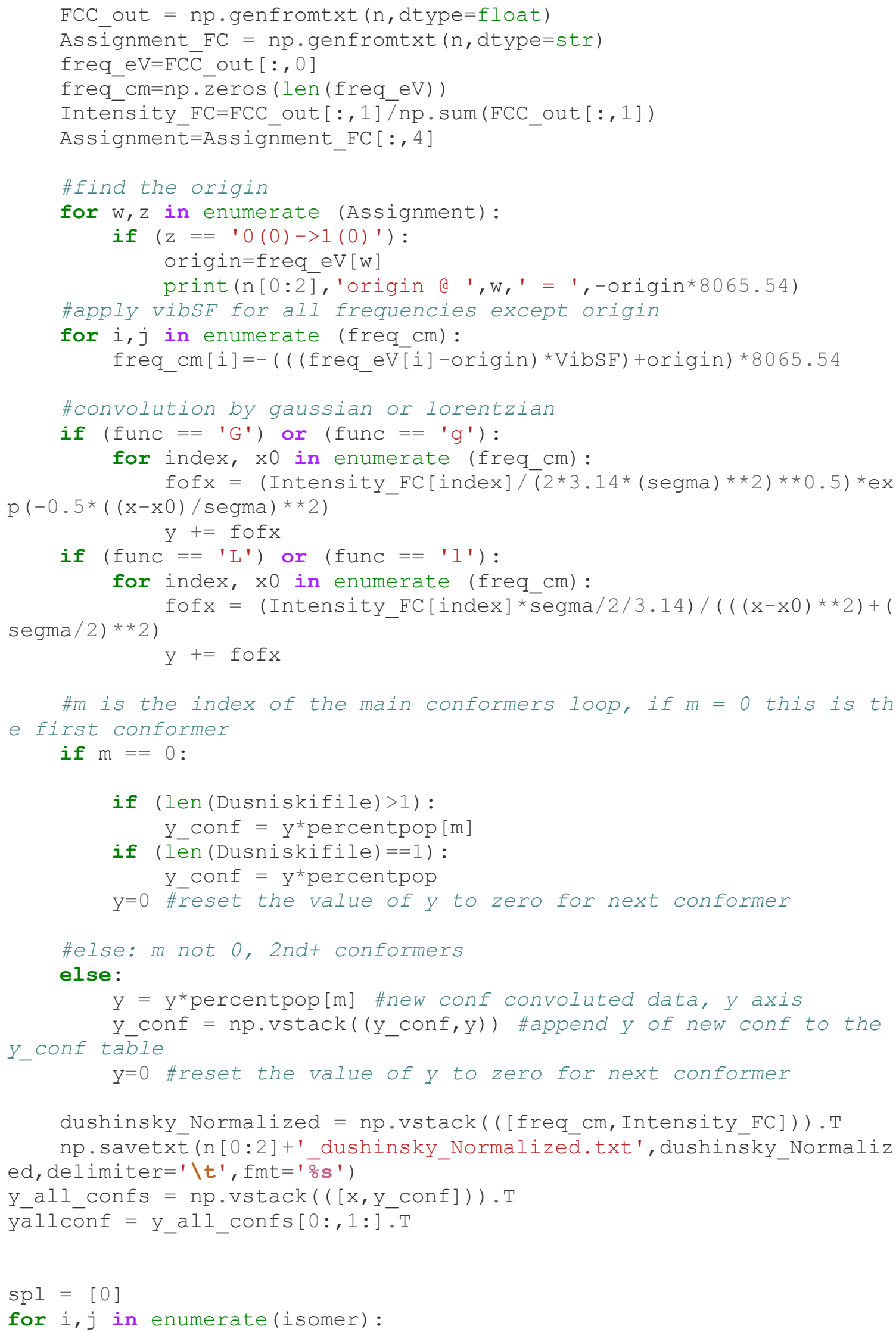




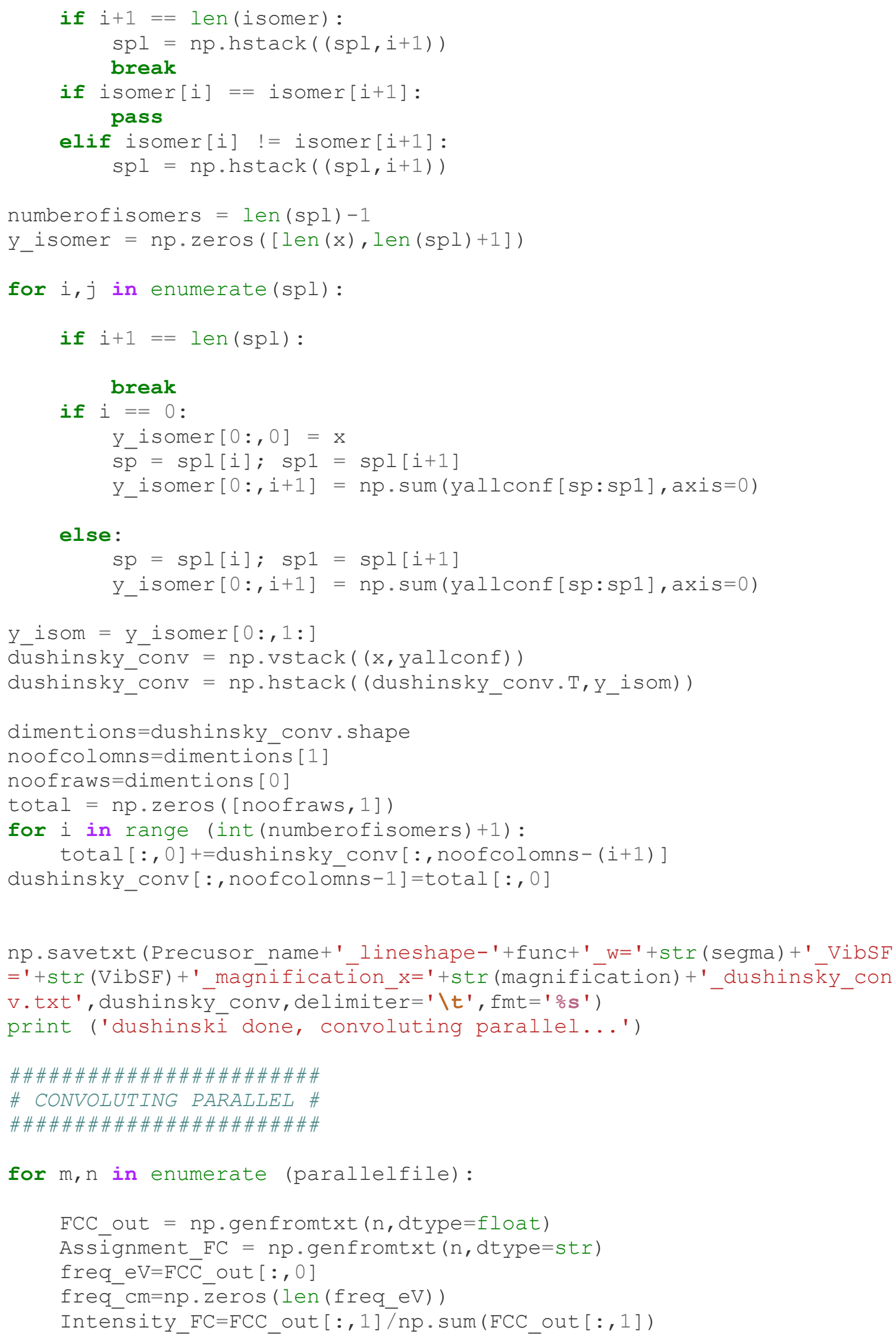




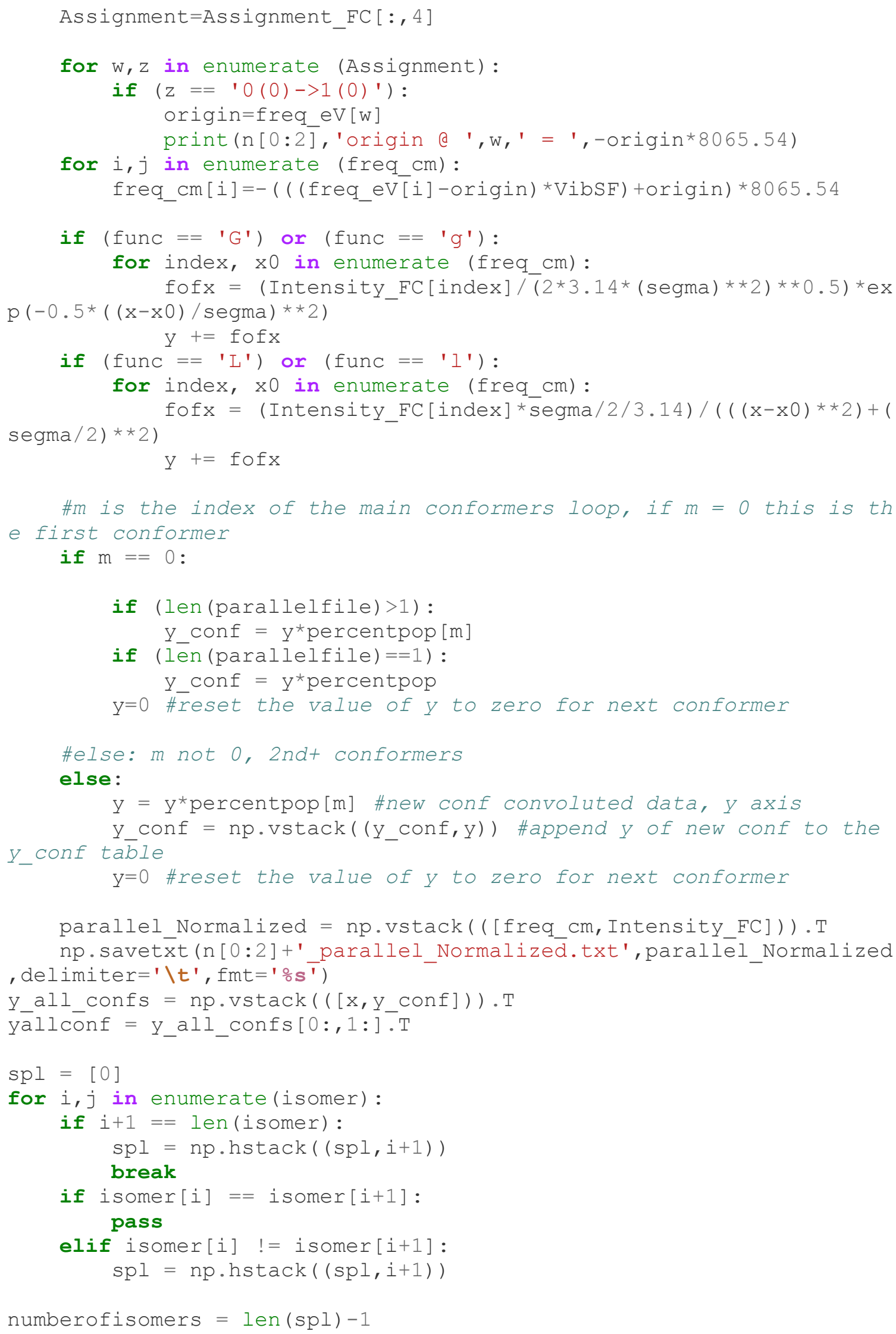




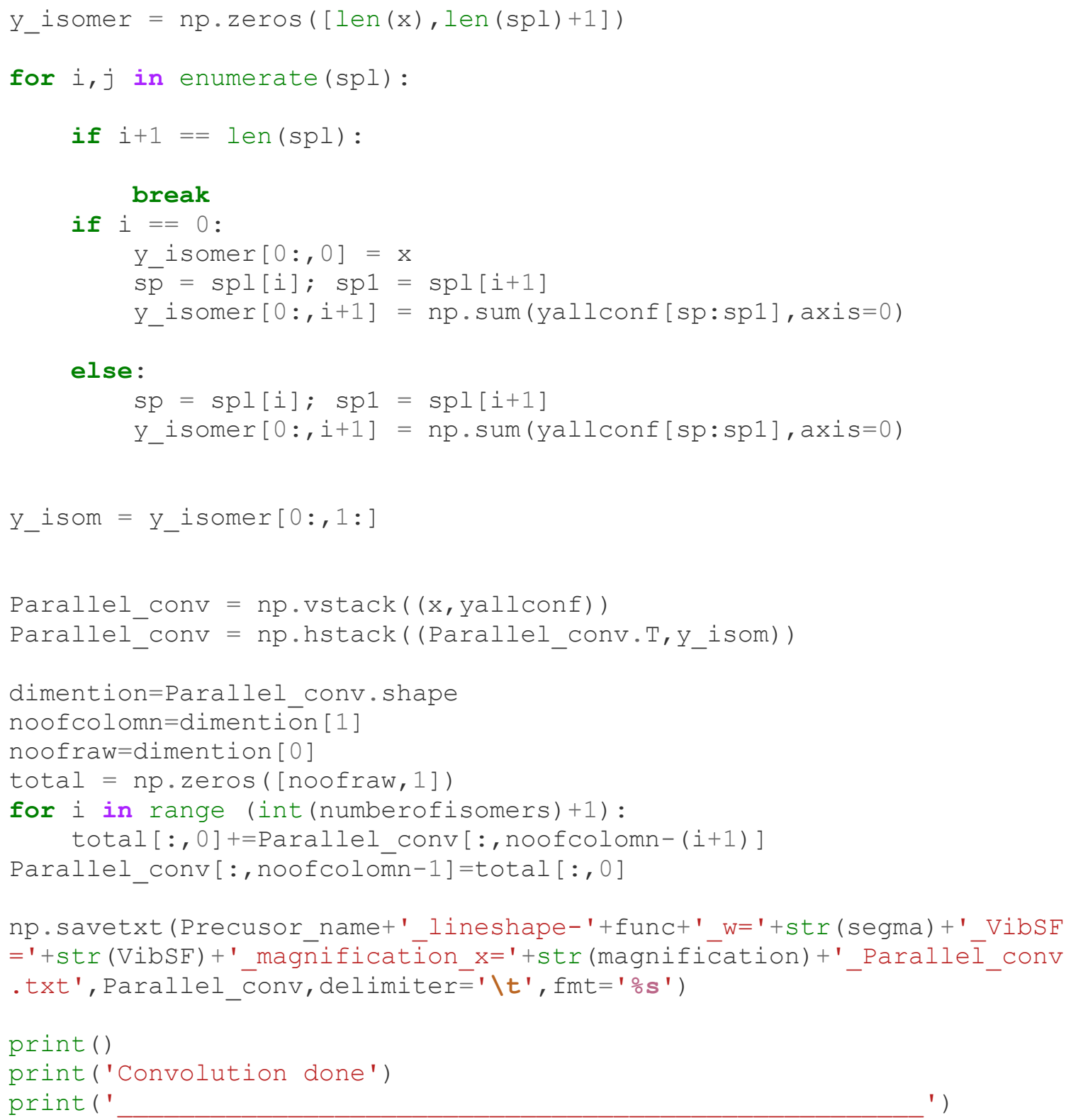




\section{APPENDIX C:}

\section{COPYRIGHT PERMISSIONS}

Figure 1.1 was reprinted with permission from (Teunis, M. B.; Jana, A.; Dutta, P.; Johnson, M. A.; Mandal, M.; Muhoberac, B. B.; Sardar, R., Mesoscale growth and assembly of bright luminescent organolead halide perovskite quantum wires. Chemistry of Materials 2016, 28 (14), 5043-5054). Copyright (2016) American Chemical Society

Figure 3.13 was adapted with permission from (Teunis, M. B.; Jana, A.; Dutta, P.; Johnson, M. A.; Mandal, M.; Muhoberac, B. B.; Sardar, R., Mesoscale growth and assembly of bright luminescent organolead halide perovskite quantum wires. Chemistry of Materials 2016, 28 (14), 5043-5054). Copyright (2016) American Chemical Society

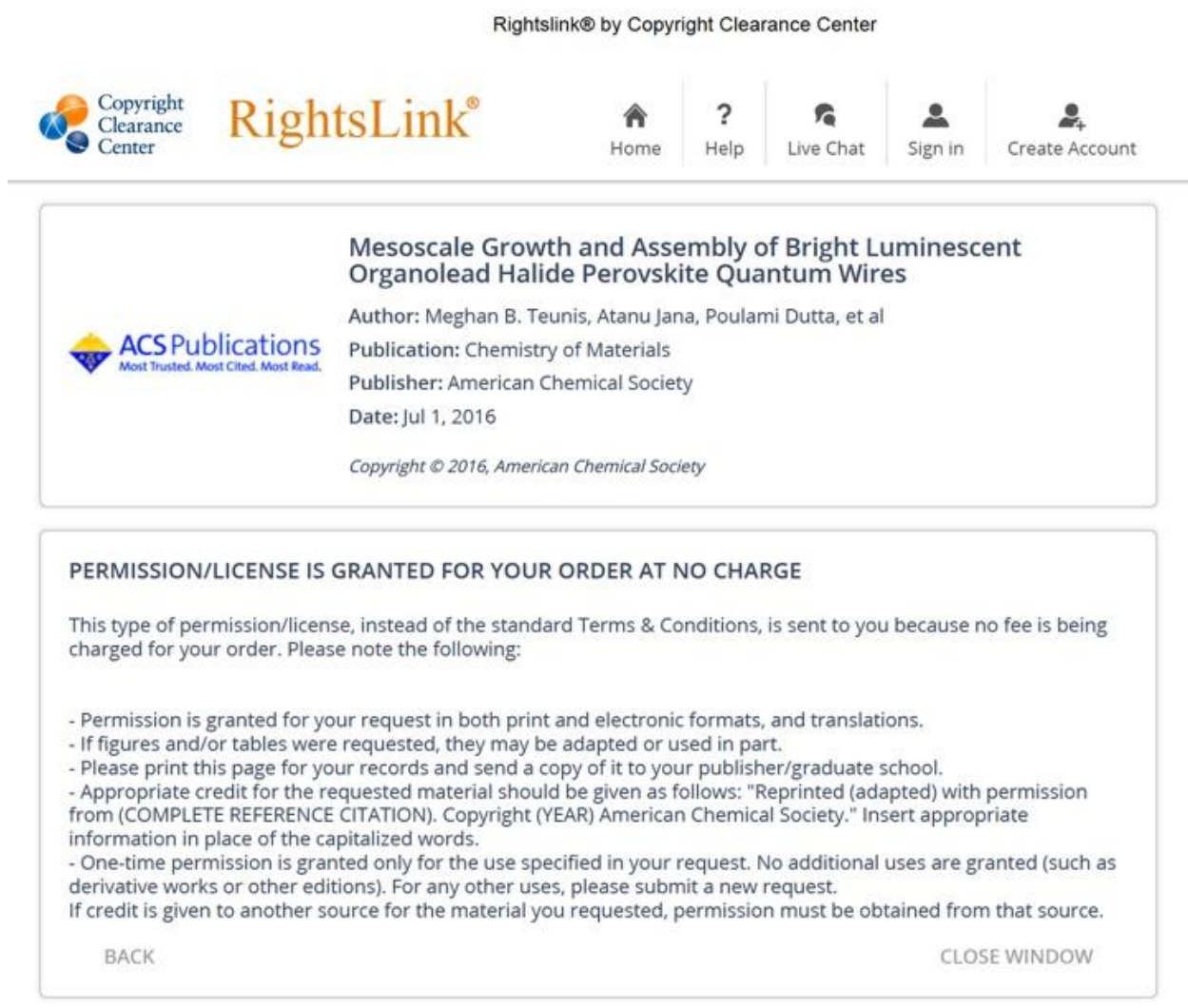


Thanks to the American Chemical Society for letting me republish my own work. In this theses, two publications of mine were presented in full with Permissions.

Reprinted with permission from (Telfah, H.; Jamhawi, A.; Teunis, M. B.; Sardar, R.; Liu, J., Ultrafast exciton dynamics in shape-controlled methylammonium lead bromide perovskite nanostructures: Effect of quantum confinement on charge carrier recombination. The Journal of Physical Chemistry C 2017, 121 (51), 28556-28565). Copyright (2017) American Chemical Society

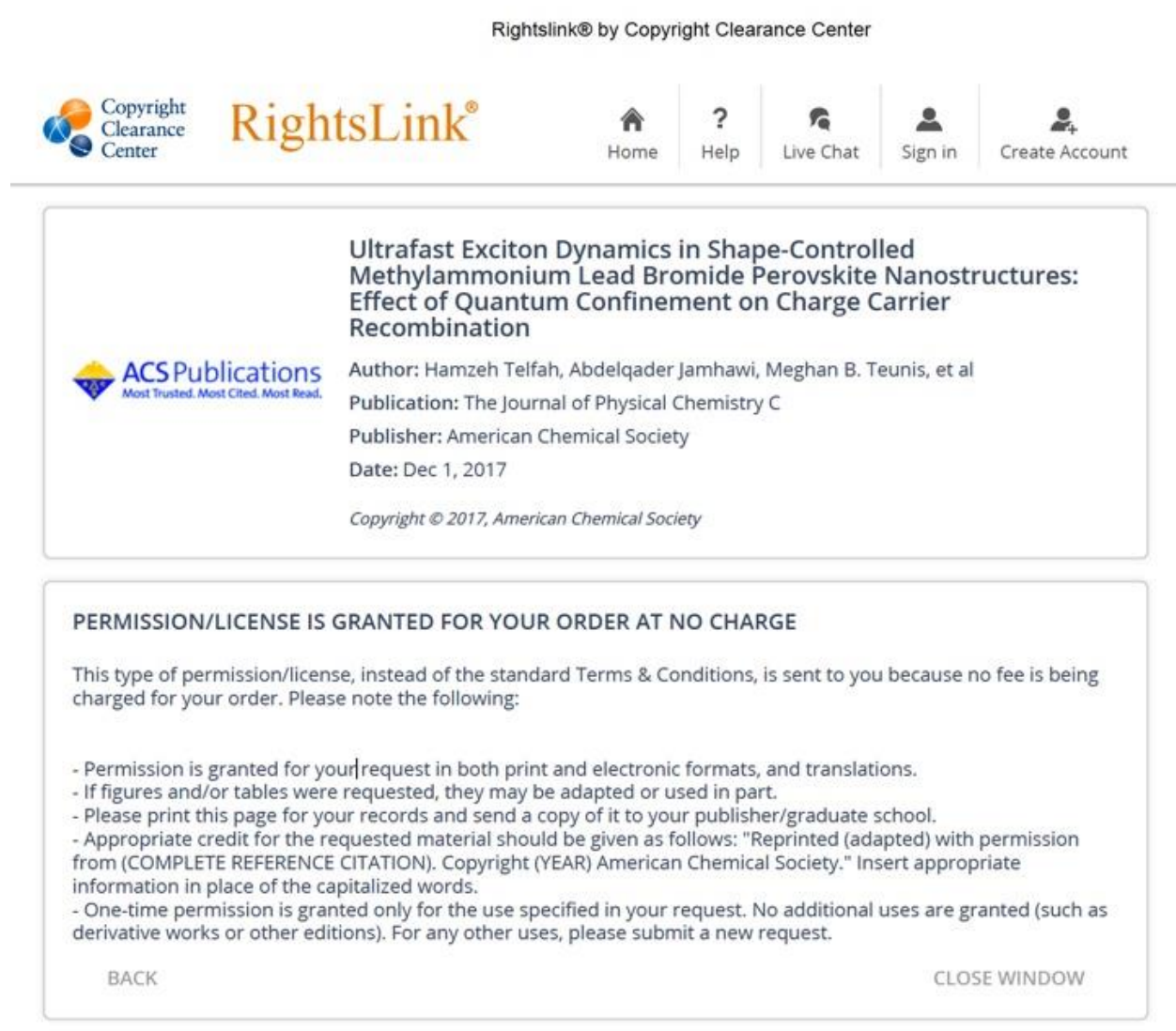

Reprinted with permission from (Telfah, H.; Reza, M. A.; Alam, J.; Paul, A. C.; Liu, J., Direct observation of tetrahydrofuranyl and tetrahydropyranyl peroxy radicals via cavity ring-down spectroscopy. The Journal of Physical Chemistry Letters 2018, 9 (16), 4475-4480). Copyright (2018) American Chemical Society 
Rightslink@ by Copyright Clearance Center

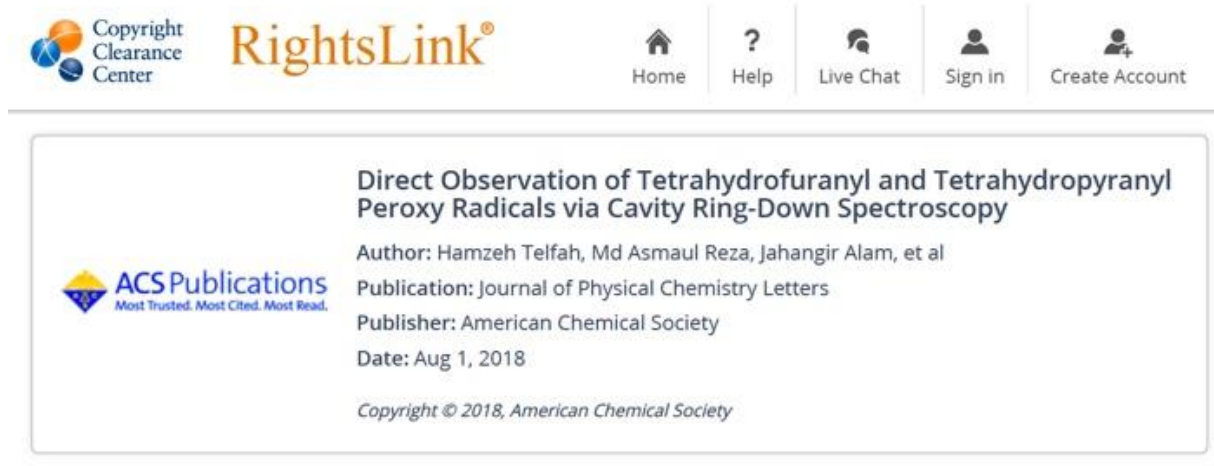

\section{PERMISSION/LICENSE IS GRANTED FOR YOUR ORDER AT NO CHARGE}

This type of permission/license, instead of the standard Terms \& Conditions, is sent to you because no fee is being charged for your order. Please note the following:

- Permission is granted for your request in both print and electronic formats, and translations.

- If figures and/or tables were requested, they may be adapted or used in part.

- Please print this page for your records and send a copy of it to your publisher/graduate school.

- Appropriate credit for the requested material should be given as follows: "Reprinted (adapted) with permission

from (COMPLETE REFERENCE CITATION). Copyright (YEAR) American Chemical Society." Insert appropriate

information in place of the capitalized words.

- One-time permission is granted only for the use specified in your request. No additional uses are granted (such as derivative works or other editions). For any other uses, please submit a new request.

BACK

CLOSE WINDOW 


\title{
CURRICULUM VITAE
}

\author{
Hamzeh Telfah \\ (502) 202-0124 \\ hamzeh.telfah@louisville.edu
}

\section{Education}

Yarmouk University / Chemistry B.S.

$2003-2007$

Jordan

Yarmouk University / Physical Chemistry, M.S.

$2008-2011$

Jordan

"Production of Biodiesel from Jordanian

Jatropha Oil"

University of Louisville Physical Chemistry, M.S.

$2014-2018$

University of Louisville Physical Chemistry, Ph.D.

$2014-2019$

"Laser Spectroscopy Investigations on

Molecular and Free Radicals Dynamics"

\section{Work Experience/Appointments}

University of Louisville / USA
Graduate Research Assistant

Graduate Teaching Assistant

- General Chem 201/202

- Physical Chem 465

SABIS International Teacher-Chemistry

Schools / UAE

- High school chemistry (12th grade)

National Chlorine QC Analyst

Industries / Jordan

Yarmouk University / Lecturer Jordan
$2014-2016$

$2016-2019$

$2012-2014$

$2011-2012$

$2008-2011$
- General Chem 105

- Physical Chem 345 


\section{Research Interests}

\section{UofL laser labs}

Investigations on material with importance in renewable energy sources and atmospheric species via high resolution and ultrafast laser spectroscopy techniques:

- Jet cooled Cavity Ring-Down Spectroscopy (CRDS) of metal-containing molecules (Publication 1-2)

- Gas-phase high-resolution laser spectroscopy of reaction intermediates (free radicals) of combustion and atmospheric species. (publications 3-5)

- CRDS and CRDS-based Reaction kinetics

- Ultrafast (femtosecond) laser spectroscopy investigations of photophysical processes and charge carrier dynamics in photovoltaic devices Nanomaterials (perovskite nanostructures). (Publication 6)

- Pump-Probe Transient Absorption (TA)

\section{Yarmouk University - Kinetics and Dynamics labs}

Investigations on Catalytic activities in the production of renewable energy sources (Biodiesel)

- Catalytic Performance in Biodiesel Production (publication 7) - ATR-FTIR

\section{Technical Skills}

- Hands-on common cw and pulsed lasers (Control, operation, maintenance)

- Pulsed YAG, Dye and Excimer lasers, single-mode cw Ti:Sa Ring laser, ring laser frequency doubling units, diode Lasers, Nonlinear optical parametric amplifiers/oscillators (NOPA, single-mode IR cw-OPO), Ultrafast lasers

- Hands-on several different scientific instruments and lab equipment

- Delay generators, Gas nozzle drivers, High voltage drivers, choppers, lockin amplifiers, photodetectors, and wavemeters, etc.

- Building spectroscopic experimental apparatus and techniques

- Optics, optomechanics optical fibers and Fabry-Perot optical cavities alignments (CRDS cavities)

- High vacuumed systems, connections and tubing connections, etc.

- Gas delivery systems, mass flow controllers, Optical tables, pneumatic isolators.

\section{Programing skills, LabVIEW and Python3}

- Data acquisition and visualization and instruments automation (LabVIEW) software package

- Created a software package for data acquisition and visualization integrated with automatic step-wise laser frequency scan, and data acquiring within scan steps supported by several other subprograms

1. Wavelength Scan control for different lasers (Sirah Dye, cw-Ring and $\left.\mathrm{cw}^{-} \mathrm{OPO}\right)$ was created and integrated into the data acquisition program.

2. Wavelength recording, and tables generation using wavemeter (HighFinesse)

3. Acquisition Instruments (Scopes, DAQ Cards) automatically detected and chosen based on the type of experiment and laser used in the experiment 
- Data manipulation and Visualization; CRD-curve fitting and LIF signal integration

- Automation and interfacing of lasers

- Optical Parametric Oscillator laser systems

- Ti:Sa Ring Laser with ring shape cavity frequency doubling unit (full control software via LabVIEW integrated with HighFinesse wavemeter)

- Subprograms for controls (using commands via PC ports - usually LabVIEW VISA) of several different scientific instruments (Oscilloscopes, Data acquisition cards, delay generators, power meters, etc.)

- Data analysis and simulations (python 3)

- Simulation of vibronic spectra Using quantum chemical calculations results (parameters extracting and population calculations, FranckCondon Factors Calculations and spectra simulation via proper line shape convolution)

\section{Experimental apparatus development}

- Two-photon CRDS Based spectroscopy apparatus

- Room temperature pulsed-CRDS

- $\mathrm{cw}^{-}$CRDS apparatus

- Adjustable Highly stabilized HR-mirrors mounts

- Jet cooled spectroscopic apparatus

- Supersonic slit/pinhole jet

- High voltage electrical discharge for the generation of target study molecules

\section{Software packages}

- Gaussian, Gauss View

- CAD software packages (Solid Works, On Shape)

\section{Conferences}

1. 253rd American chemical society, national meeting \& exposition, advanced materials, technologies, systems \& processes, San Francisco, CA. Ultrafast Transient Absorption Spectroscopy Investigations of Charge Carrier Dynamics of Methyl Ammonium Lead Bromide $\left(\mathrm{CH}_{3} \mathrm{NH}_{3} \mathrm{PbBr}_{3}\right)$ Perovskite Nanostructures.

2. $72^{\text {nd }}$ International Symposium on Molecular Spectroscopy ChampaignUrbana, Illinois, USA Ultrafast Transient Absorption Spectroscopy investigation of excited state dynamics of methylammonium lead bromide perovskite nanostructures.

3. 73rd International Symposium on Molecular Spectroscopy ChampaignUrbana, Illinois, USA, Observation of The $\widetilde{A} \leftarrow \widetilde{\mathrm{X}}$ Electronic Transitions Of Tetrahydropyranyl And Tetrahydrofuranyl Peroxy Radicals By RoomTemperature Cavity Ring-Down Spectroscopy.

4. Cavity Ring-down Spectroscopy of 1-, 2- and 3-methylallyl peroxy radicals. 73rd International Symposium on Molecular Spectroscopy, ChampaignUrbana, Illinois, USA. June 2018 


\section{Publications}

1. "Cavity ring-down spectroscopy of jet-cooled YO molecules: laser cooling molecules" A. C. Paul, $\underline{\text { H. Telfah, J. Liu, }{ }^{*} \text {, In Preparation. }}$

2. "Laser-induced fluorescence and dispersed-fluorescence spectroscopy of the $\mathrm{A}^{2} \mathrm{E}-\mathrm{X}^{2} \mathrm{~A}_{1}$ transition of jet-cooled calcium methoxide $\left(\mathrm{CaOCH}_{3}\right)$ Radicals"

A. C. Paul, K. Sharma, Md. A. Reza, H. Telfah, T. A. Miller, ${ }^{*}$ and J. Liu, ${ }^{*} J$. Chem. Phys. 151.13 (2019): 134303.

3. "Revealing long-range substituent effects in the laser-induced fluorescence and dispersed fluorescence spectra of jet-cooled $\mathrm{CH}_{\mathrm{x}} \mathrm{F}_{3-\mathrm{x}} \mathrm{CH}_{2} \mathrm{O}$ $(\mathrm{x}=1,2,3)$ Radicals", B. Koncz, G. Bazsó, Md A. Reza, H. Telfah, K. Hegedus, J. Liu, ${ }^{*}$ G. Tarczay, ${ }^{*}$ J. Phys. Chem. A (submitted).

4. "Room-temperature cavity ring-down spectroscopy of methyl-allyl peroxy radicals", Md. A. Reza, $\underline{\mathrm{H}}$. Telfah, R. Xu, and J. Liu, * J. Phys. Chem. A 123, pp 3510-3517 (2019).

5. "Direct observation of tetrahydrofuranyl and tetrahydropyranyl peroxy radicals via cavity ring-down spectroscopy", $\underline{\mathbf{H}}$. Telfah, Md. A. Reza, J. Alam, A. C. Paul and J. Liu, * J. Phys. Chem. Lett. 9, 4475-4480 (2018).

6. "Ultrafast exciton dynamics in shape-controlled methylammonium lead bromide Perovskite nanostructures: Effect of quantum confinement on charge carrier recombination", H. Telfah, A. Jamhawi, M. B. Teunis, R. Sardar, and J. Liu,* J. Phys. Chem. C 121, 28556-28565 (2017).

7. "Effect of Thermal Treatment on the Catalytic Performance of Magnesium Oxide-Hydroxide Systems in Biodiesel Production from Jatropha Oil:

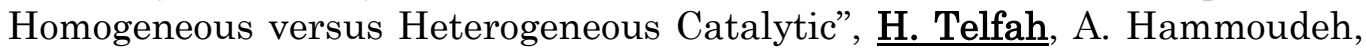
S. Mahmoud, Jordan Journal of Chemistry, 13, 93-102 (2018)

\section{Mentoring}

I have mentored two chemistry undergraduate students, Mr. Noman Farooq and Mr. Robert Gardner from Chemistry Department at University of Louisville and one visiting graduate student Ms. Gong Ting from Shanxi University - China.

\section{Volunteer work}

- Management and maintenance of a new computer laboratory which was donated to the Chemistry department at Yarmouk university by Japan international cooperation agency (JICA) - Jordan.

- Research in drug delivery and supramolecular chemistry United Arab Emirates University / UAE.

- Other Community services (cleaning and mowing)

- Session chair helper in the International Symposium on molecular spectroscopy. 